\title{
Multipactor in Multicarrier Systems Theory and Prediction
} Sergio Anza Hormigo

Directores:

aurora Software and Testing, S.L., Dr. Carlos P. Vicente Quiles Universitat de València, Dr. Benito Gimeno Martínez Universitat Politècnica de València, Dr. Vicente E. Boria Esbert

Tesis Doctoral presentada en la Universidad Politécnica de Valencia para la obtención del título de Doctor Ingeniero de Telecomunicación.

Valencia, Junio 2014 

A mis padres, a mis hijos, Darío y Marco. 



\section{Agradecimientos}

Los inicios de esta tesis doctoral se remontan allá por el 2006 cuando Vicente Boria me ofreció la oportunidad de hacer el doctorado dentro una empresa tecnológica valenciana que acababa de nacer: Aurorasat. Por circunstancias de la vida, en ese momento yo estaba embarcado en otro proyecto profesional. Pero Vicente, como siempre, fue muy persuasivo y finalmente me convenció para unirme a ellos. De todas las decisiones que he tomado, en todos los ámbitos de mi vida, ésta creo que ha sido una de las más acertadas. Vicente, te doy las gracias por confiar en mí desde el principio y permitirme poder unirme al grupo fantástico que integra Aurorasat donde he trabajado, disfrutado y crecido en el plano profesional y personal durante estos últimos años.

Fruto de esta relación ha nacido esta tesis doctoral, que ha tenido una larga gestación de casi 8 años. Desde el instante $t=0$, Carlos Vicente ha sido una referencia para mí, tanto por su inagotable conocimiento científico, como por su capacidad de trabajo, intuición y liderazgo. Carlos, por todo esto y por ser una gran persona y mejor amigo, te doy las gracias.

Durante todo este tiempo, Benito Gimeno me ha ofrecido su inestimable apoyo y guía. Benito, como buen físico, siempre has apostado por sacar mi lado científico y por usar rigurosa formulación teórica, algo que los ingenieros siempre estamos gustosos a dejar de lado si existe alguna herramienta más práctica. Gracias por tu confianza y amistad.

Otro gran protagonista es Jordi Gil, que aunque no ha di- 
rigido oficialmente este trabajo, siempre ha sido el primero en interesarse por él y en darme empujones cuando hacían falta. Al final, incluso se ofreció a corregir capítulos de la tesis "por placer", lo que dice mucho de su alta implicación (o de su masoquismo extremo). Gracias Jordi por tu apoyo, y por ayudar a que este trabajo se entienda un poquito mejor. Eres un gran profesional y amigo.

Quiero también dar las gracias a Michael Mattes de la École Polytechnique Fédérale de Lausanne, por sus interesantes discusiones científicas y contribuciones a este trabajo.

En el aspecto personal, siempre me he quejado de lo larga que se me estaba haciendo esta tesis. Pero a decir verdad el tiempo se me ha pasado volando. Eso es gracias a que todos los días he ido con ilusión y ganas al trabajo. Estoy rodeado de grandes profesionales y amigos en lo que considero ya como una segunda familia. No tengo espacio para escribir las líneas que debería dedicarle, por justicia, a cada uno de los integrantes de Aurorasat, que han vivido mi tesis como parte de lo cotidiano. Me limito a citar sus nombres, en un profundo gracias, a los que están y estuvieron: Jaime, Teresa, María, Carmen, Javi, Mario, Carlos G., Carlos C., Esteban, Iñaki, Javi Monge, et al.

Sería injusto no mencionar a los compañeros del IEEC de Barcelona de los que guardo un gran recuerdo: Carlo, Anna, Alina, Moi, Miquel, Silvia, Violeta, Pep, Josep, Jose, Oleguer, Santi y Estel entre otros. Un abrazo a todos ellos.

He tenido suerte de tener buenos amigos y familiares que me han apoyado con paciencia y comprensión en esta aventura. Todos los ánimos y los buenos momentos que me habéis dado han sido fundamentales para llegar a buen puerto. Gracias a todos. Gracias también a Yolanda por apoyarme desde el principio y durante largo tiempo.

Quiero expresar un agradecimiento muy especial a mis padres. Obviamente sin vosotros no sería lo que soy ahora, y me habéis dado todo el cariño, apoyo y confianza imaginables. Os doy las gracias por el espíritu curioso e independiente que me habéis 
inculcado. Me habéis enseñado a ser crítico, a razonar por mi mismo y a preguntarme el por qué de las cosas. Sois mi más temprana motivación para dedicarme a la investigación. Gracias.

Finalmente quiero dedicarle unas palabras a mis hijos.

Darío y Marco.

Hacéis que la vida sepa mejor.

Sed vosotros, sed felices.

Os quiero. 



\section{Resumen}

Este trabajo presenta un nuevo marco teórico y una nueva herramienta de predicción del fenómeno de multipactor para sistemas multi-portadora. Este estudio es de capital importancia para aplicaciones de comunicaciones por satélite, donde existe cada vez mayor demanda del número de canales operando a alta potencia. Las herramientas de predicción de multipactor ayudan a reducir o eliminar completamente el riesgo de que ocurra una descarga de RF en operación, con la correspondiente pérdida de calidad de la señal o incluso la completa ruptura del componente.

Actualmente, se conoce poco sobre el fenómeno de multipactor con señales multi-portadora. Esta Tesis presenta una nueva teoría no estacionaria para señales multi-portadora, basada en estudios actuales de multipactor estadístico. Al contrario que las teorías existentes, la nueva teoría propuesta es capaz de modelar ambos procesos de creación y absorción de electrones. Por lo tanto, representa la primera teoría de multipactor para señales multi-portadora que es totalmente capaz de caracterizar la dinámica de los electrones.

A su vez, se presenta un nuevo método de predicción, el método quasiestacionario, que encuentra automáticamente la combinación de fases de la señal multi-portardora que implican un nivel de descarga más bajo. Este método se basa en la teoría no estacionaria de multipactor unido a un optimizador global genético. La predicción quasi-estacionaria funciona para señales con número de portadoras arbitrario a frecuencias arbitrarias. El método quasi-estacionario se ha contrastado con medidas experimentales en filtros en guía de onda rectangular en banda $\mathrm{Ku}$, donde se han usado diferentes tipos de señales. El método quasi-estacionario ofrece predicciones más precisas que la "20-gap-crossing rule". Los errores de predicción han resultado ser de $1 \mathrm{~dB}$ y $4 \mathrm{~dB}$, respectivamente.

En principio, aunque la teoría no-estacionaria está formulada para el caso unidimensional de placas plano-paralelas, ésta se puede adaptar a otras geometrías y señales. Por lo tanto, esto abre un línea de investigación futura que puede extender la teoría a aplicaciones más complejas. 



\section{Resum}

Aquest treball presenta un nou marc teòric i una nova eina de predicció del fenomen de multipactor per a sistemes multi-portadora. Aquest estudi és de cabdal importància per a aplicacions de comunicacions per satèl-lit, on existeix cada vegada major demanda del nombre de canals operant a alta potència. Les eines de predicció de multipactor ajuden a reduir o eliminar completament el risc que ocórrega una descàrrega de RF en operació, amb la corresponent pèrdua de qualitat del senyal o fins i tot la completa ruptura del component.

Actualment, es coneix poc sobre el fenomen de multipactor amb senyals multi-portadora. Aquesta Tesi presenta una nova teoria no estacionària per a senyals multi-portadora, basada en estudis actuals de multipactor estadístic. Al contrari que les teories existents, la nova teoria proposada és capaç de modelar tots dos processos de creació i absorció d'electrons. Per tant, representa la primera teoria de multipactor per a senyals multiportadora que és totalment capaç de caracteritzar la dinàmica dels electrons.

$\mathrm{Al}$ seu torn, es presenta un nou mètode de predicció, el mètode quasiestacionari, que troba automàticament la combinació de fases del senyal multi-portardora que impliquen un nivell de descàrrega més baix. Aquest mètode es basa en la teoria no estacionària de multipactor unit a un optimizador global genètic. La predicció quasi-estacionària funciona per a senyals amb nombre de portadores arbitrari a freqüències arbitràries. El mètode quasi-estacionari s'ha contrastat amb mesures experimentals en filtres en guia d'ona rectangular en banda $\mathrm{Ku}$, on s'han usat diferents tipus de senyals. El mètode quasi-estacionari ofereix prediccions més precises que la "20-gap-crossing rule". Els errors de predicció han resultat ser d'1 dB i $4 \mathrm{~dB}$, respectivament.

En principi, encara que la teoria no-estacionària està formulada per al cas unidimensional de plaques pla-paral-leles, aquesta es pot adaptar a altres geometries i senyals. Per tant, açò obri un línia de recerca futura que pot estendre la teoria a aplicacions més complexes. 



\section{Abstract}

This work presents a new theoretical framework and prediction tool for multipactor in multi-carrier systems. This is of capital importance for satellite communication applications, which demand for higher number of channels operating at high power levels. Such a tool may help to reduce, or completely avoid, the risk of having an RF breakdown in operation, with the subsequent loss of signal quality or even completely damage of the device.

Currently not much is known about multipactor for multi-carrier signals. This Thesis throws light on some basic multipactor mechanisms such as the electronic discharge build-up or its connection with signal distortion. A new non-stationary multi-carrier theory, based on current statistical multipactor studies, is presented. Unlike existing ones, this theory is able, for the first time, to model both electron creation and absorption processes. It constitutes the first multipactor theory for multi-carrier signals which is able to fully characterize the electron dynamics, such as the time evolution of the electronic density, as well as creation and absorption rates.

A prediction method, the quasi-stationary method, is proposed for the automatic searching of the combination of carrier phases which yields the lowest breakdown level. It is based on the non-stationary theory for singlecarrier signals together with a genetic global optimizer. The quasi-stationary prediction method returns the worst-case phase combination plus a breakdown level for arbitrary multi-carrier signals with any number of carriers. The quasi-statinary method has been assessed with experimental tests on ad-hoc Ku-band rectangular waveguide samples with different multi-carrier signals. Additionally, the results have been contrasted with the popular 20 -gap-crossing rule. The quasi-stationary method yields much better prediction accuracy than the 20-gap-crossing rule. Prediction errors for both techniques have been found to be $1 \mathrm{~dB}$ and $4 \mathrm{~dB}$, respectively.

The non-stationary theory is formulated for one-dimensional parallelplate case, but in principle can be adapted to other geometries and signals, which opens a future research line for extending it to more complex applications. 



\section{Contents}

Contents

1 Introduction 1

1.1 Thesis objectives and structure . . . . . . . . . 4

2 The multipactor phenomenon $\quad 7$

2.1 Secondary Electron Emission effect . . . . . . . . . 12

2.2 Saturation . . . . . . . . . . . . . . . 18

2.3 Single-carrier and multi-carrier signals . . . . . . . 20

2.4 State of the art . . . . . . . . . . . . . 22

2.4.1 Necessary conditions . . . . . . . . . . . . 22

2.4 .2 Techniques against Multipactor . . . . . . . . 22

2.4 .3 Multipactor design and test . . . . . . . . . . 24

2.4.3.1 Multipactor prediction ....... 24

2.4.3.2 Multipactor test . . . . . . . . 27

3 Article index 31

3.1 Long-term multipactor discharge in multicarrier systems 31

3.1.1 Bibliographic record . . . . . . . . . . . . 31

3.1.2 Abstract . . . . . . . . . . . . . . 32

3.2 RF breakdown prediction for microwave passive components in multi-carrier operation . . . . . . . . . . 32

3.2.1 Bibliographic record . . . . . . . . . . . . . 32

$3.2 .2 \quad$ Abstract . . . . . . . . . . . . . . . 32

3.3 Non-stationary Statistical Theory for Multipactor . . . 33

3.3 .1 Bibliographic record . . . . . . . . . . . 33

$3.3 .2 \quad$ Abstract . . . . . . . . . . . . . . 33 
3.4 Multipactor theory for multi-carrier signals . . . . . . 34 3.4 .1 Bibliographic record . . . . . . . . . . . . 34

3.4 .2 Abstract. . . . . . . . . . . . . 34

3.5 Prediction of Multipactor Breakdown for Multi-carrier Applications: The Quasi-stationary Method . . . . . . 35

3.5.1 Bibliographic record . . . . . . . . . . . 35

3.5 .2 Abstract. . . . . . . . . . . . 35

4 Article list $\quad 37$

4.1 Long-term multipactor discharge in multicarrier systems 37

4.1 .1 Introduction . . . . . . . . . . . . 37

4.1.2 Multicarrier multipactor basics . . . . . . . . 40

4.1.3 SEY model . . . . . . . . . . . . . . . . . 42

4.1.4 Accumulation model . . . . . . . . . . . . . . 44

4.1 .5 Numerical results . . . . . . . . . . . . . . . . . 49

4.1.5.1 Single carrier signal . . . . . . . . . 51

4.1.5.2 Pulsed signal . . . . . . . . . . . . 51

4.1.5.3 Multicarrier signal . . . . . . . . . 54

4.1 .6 Conclusions . . . . . . . . . . . . . . 55

4.2 RF breakdown prediction for microwave passive components in multi-carrier operation . . . . . . . . . 57

4.2 .1 Introduction . . . . . . . . . . . . 57

4.2 .2 Multipactor Basics . . . . . . . . . . . . . 57

4.2.3 Field Analysis of Microwave Devices . . . . . . 59

4.2.4 Electron Motion and Space Charge Computation 61

4.2 .5 Results . . . . . . . . . . . . . . 62

4.2 .6 Conclusions . . . . . . . . . . . . . 65

4.3 Non-stationary Statistical Theory for Multipactor . . . 67

4.3.1 Introduction . . . . . . . . . . . . 67

4.3.2 Limitations of the current theories . . . . . . . 68

4.3.3 Non-stationary Statistical Multipactor . . . . . 72

4.3.3.1 Equations of motion . . . . . . . . . 73

4.3.3.2 Definitions and conventions . . . . . . 74

4.3.3.3 Statistical development . . . . . . . . 75

4.3.3.4 Theoretical Analysis . . . . . . . . . . 82

4.3.4 Numerical Simulations . . . . . . . . . . . . . . 84

4.3 .5 Results . . . . . . . . . . . . . 86

4.3 .6 Conclusions . . . . . . . . . . . . . . . . . 89 
4.4 Multipactor theory for multi-carrier signals . . . . . . 91

4.4 .1 Introduction . . . . . . . . . . . . . . . . 91

4.4 .2 Multi-carrier signal analysis . . . . . . . . . . 93

4.4.3 Multipactor theory for multi-carrier signals . . 99

4.4.3.1 Equations of motion . . . . . . . . . 99

4.4.3.2 Multipactor theory for multi-carrier signals . . . . . . . . . 101

4.4.4 Numerical Simulations . . . . . . . . . . . . . . 110

4.4.5 Results ................. . 111

4.4.5.1 Zero phases - uniform frequency spacing 111

4.4.5.2 Triangular phases - uniform frequency spacing .............. 115

4.4.5.3 Arbitrary phases - non-uniform frequency spacing and different amplitudes 116

4.4 .6 Conclusions . . . . . . . . . . . . . . . . . . 117

4.5 Prediction of Multipactor Breakdown for Multi-carrier

Applications: The Quasi-stationary Method . . . . . . 119

4.5.1 Introduction . . . . . . . . . . . . . 119

4.5.2 Background . . . . . . . . . . . . . . . . . . . 122

4.5.2.1 Multipactor in multi-carrier signal . . 122

4.5.2.2 The 20-gap-crossing rule . . . . . . . 125

4.5.2.3 Parallel-plate geometry . . . . . . . 126

4.5.3 Quasi-stationary model prediction . . . . . . 127

4.5.3.1 Theory . . . . . . . . . . . . 128

4.5.3.2 Procedure for threshold determination 132

4.5.4 Testing . . . . . . . . . . . . . . . 134

4.5.4.1 Frequency plans and tests . . . . . . 135

4.5.4.2 Sample Design . . . . . . . . . . . . 135

4.5.4.3 Description of the test bed . . . . . 137

4.5.4.4 SEY measurements . . . . . . . . 138

4.5.5 Simulation software . . . . . . . . . . . . 140

4.5.6 Predictions and test results . . . . . . . . . . 140

4.5.6.1 Error definition . . . . . . . . . . 140

4.5.6.2 Phase configurations . . . . . . . . . . 141

4.5.6.3 RF breakdown prediction and measurements . . . . . . . . . . . 142

4.5.6.4 Analysis of results . . . . . . . . . 148

4.5 .7 Conclusions . . . . . . . . . . . . . . . . . . 148 
5 General Discussion 151

5.1 Single-carrier theories . . . . . . . . . . . . . . 151

5.1 .1 Classical multipactor theory . . . . . . . . . . 151

5.1.1.1 Constant- $k$ theory . . . . . . . . . . 153

5.1.1.2 Constant- $v$ theory . . . . . . . . . 156

5.1.1.3 Hybrid modes . . . . . . . . . . . . 157

5.1.1.4 Phase focusing . . . . . . . . . . . 159

5.1.2 Statistical Multipactor theories . . . . . . . . . . 161

5.1.2.1 Stationary theory . . . . . . . . . 163

5.1.2.2 Non-stationary theory . . . . . . . . . 169

5.1.3 Single-carrier prediction . . . . . . . . . . . 170

5.2 Multi-carrier theories . . . . . . . . . . . . . . . . 171

5.2 .1 Peak power . . . . . . . . . . . . . 172

$5.2 .2 \quad 20$-gap-crossing rule . . . . . . . . . . . 173

5.2.3 Non-stationary multi-carrier theory . . . . . . . 177

5.2.4 Quasi-stationary theory . . . . . . . . . . 178

5.2.5 Multi-carrier prediction: Worst-case determination . . . . . . . . . . . . . 179

5.3 Discussion on experimental results . . . . . . . . 185

6 Conclusions and future work 191

6.1 Conclusions . . . . . . . . . . . . . . . . . 192

6.2 Future work . . . . . . . . . . . . . . . . . 195

A Electron Seeding Techniques 199

A.1 Radioactive sources . . . . . . . . . . . . . . . . . 200

A.2 UV lamps . . . . . . . . . . . . . . . . . . 204

A.3 Regulated Electron Gun . . . . . . . . . . . . 207

$\begin{array}{ll}\text { B Demonstration } 1 & 209\end{array}$

$\begin{array}{ll}\text { Bibliography } & 215\end{array}$ 


\section{Chapter 1}

\section{Introduction}

Microwave breakdown is one of the most common power handling limitations in radio-frequency (RF) systems. High power levels imply the presence of high level electromagnetic fields in the devices which may cause two known effects: The Corona effect, when the device is filled with any kind of gas, and the Multipactor effect, when operating in vacuum conditions.

The Corona effect consists in the ionization of the gas molecules inside a device produced by high power electromagnetic fields. The electrons in the outer shells of the gas molecules are released and interact with other molecules releasing more electrons. Such effect produces an increase of electronic charge density that implies a signal distortion and ultimately a destructive electronic avalanche. The Corona breakdown level depends among many other parameters on the kind of gas and the pressure level inside the device.

As pressure decreases towards vacuum, or near vacuum conditions, the electron mean free path exceeds the dimensions of the device. In this situation, free electrons, which are accelerated by the electromagnetic fields, can move without opposition until striking any surface of the component. If the impact energy is high enough, secondary electrons may be released from the surface. Then, if the 
resulting electronic bunch enters in resonance with the field, it will impact periodically with the device surfaces releasing more and more electrons, which ultimately produces an electron avalanche. This phenomenon is known as Multipaction, Multipactoring or Multipactor effect.

Even if Multipactor and Corona effects share basic principles, their study is completely different. Corona is a volumetric phenomenon which depends mainly on gas ionization and absorption rates, whereas Multipactor is heavily dependent on the geometry of the device, the resonance between field and electron motion, and the surface properties of the material. As a consequence both phenomena usually appertain to different research lines. Also, their field of application is different, although with little overlapping. Corona applies to areas in which the high power electronic equipment works in pressurized ambients, such as terrestrial communication systems. On the other hand, the Multipactor phenomenon is related with applications in which high power systems operate in vacuum, such as satellite communication systems and particle accelerators. Nevertheless, in certain space communication systems there may be both Corona and Multipactor risk. For example, during the launching of a satellite, communications may be done while traversing the Earth atmosphere and, therefore, some high power devices are working in a low pressure environment, increasing the risk of a Corona discharge.

In short, and now focusing exclusively on Multipactor, it is an undesired high power phenomenon, which may appear in many kinds of technologies and circuits: microstrip and strip lines, waveguides, coaxial lines and connectors, as well as active components and antennas. A multipactor discharge produces heat, noise, power reflection and signal distortion which negatively affects the correct operation of the device. Ultimately, strong multipactor discharges produce outgassing and a local increase of pressure that initiates a Corona discharge and may permanently damage the component.

It is, thus, evident that the design of multipactor free compo-

${ }^{1}$ Those in which operation power is below multipactor breakdown power nents $^{1}$ is desirable for every application. However, for the space industry, this becomes a key issue, since a failure of a single component in operation may imply the failure of the whole mission, and the subsequent loss of enormous amounts of time and money.

Multipactor tests in Earth laboratories are used to validate com- 
ponents to be multipactor free up to the desirable power. Unfortunately, these tests are very costly and a try-and-error design approach $^{1}$ is unaffordable.

On the other hand, Multipactor prediction methods, which rely on the theoretical study of the Multipactor phenomenon, and numerical simulation software tools, allow for establishing the Multipactor breakdown of a certain component in the design phase, thus reducing the experimental testing to the minimum. For this reason, Multipactor prediction is a must in the component design for high-power space applications.

The classical Multipactor theory only covers the single-carrier case, i.e. systems in which only one carrier is transmitted. For singlecarrier operation, theoretical prediction methods as well as software tools provide reliable results that have been successfully contrasted in the past three decades. However, in satellite applications, the normal situation is to operate with a high number of communication channels with a a high power level per carrier. This produces extremely high peak power levels in the multi-carrier path of the spacecraft, and therefore a high risk of Multipactor discharge.

Besides the publications that belong to this thesis ${ }^{2}$, no multicarrier theory is available in the literature. The existing prediction methods, such as the peak-power or 20-gap-crossing rule, are based in very rough simplifications, sometimes without enough physical ba$\mathrm{sis}^{3}$. The resulting predictions coming from these methods are usually too conservative and imply high design margins that result in over-designed, heavy components.

On the other hand, numerical simulators give satisfactory predictions for single-carrier operation. But, many more parameters are involved and very heavy computations are needed in the multicarrier counterpart ${ }^{4}$. Therefore they provide accurate breakdown calculations for particular parameter combinations but are not able to simulate all possible combinations in order to provide the worst case prediction.

Therefore, the main motivation of this thesis has been to develop the first full multipactor multi-carrier theory and to propose a multicarrier prediction method that is able to provide accurate predictions. Hopefully, this work will contribute to the scientific community with a better understanding of the multipactor phenomenon. At the same

${ }^{3}$ This is reviewed with more detail in Chapter 5

${ }^{4}$ The breakdown level depends on the amplitude and phase of all carriers in the signal 
time, it is expected that this work will help the industry to reduce design margins and test campaigns, with the consequent cost and time savings.

This Thesis has been done in the framework of the European Space Agency (ESA) activity AO/1-4978/05/NL/GLC: MULTIPACTOR IN MULTICARRIER RF SYSTEMS, which consisted in the study of multipactor in multi-carrier operation. In this activity, theoretical and numerical results have been crosschecked with experimental measurements. These data have been already published and will also be presented as part of this Thesis. This research has been also partially supported by the Ministerio de Ciencia e Innovacion (Spain) through the "Programa Torres Quevedo" PTQ06-2-0693

\subsection{Thesis objectives and structure}

The purpose of this work is to construct a reliable full multipactor theory covering both single and multi-carrier cases. This theory will be the basis for a new prediction method which takes into account the most relevant parameters involved in the multipactor discharge, such as the envelope shape, SEY properties of the device surfaces, as well as single-events and long-term multi-carrier discharges. A solid multipactor criterion will be also established.

This theory will be formulated for the two-surface parallel-plates one-dimensional multipactor case, since it is the most simple and representative of all cases. The extension of this theory to more complicated geometries, such as rectangular, circular or coaxial waveguides, microstrip lines and antennas, is left for future work.

This development is done in different steps:

- Develop a non-stationary single-carrier theory which predicts accurately experimental breakdowns and also models the electron multiplication and absorption processes experienced during the multipactor discharge.

- Develop a general non-stationary statistical multipactor theory for multicarrier signals based on the previous single-carrier theory. 
- Develop new multicarrier prediction models based on the new non-stationary statistical multipactor theory.

- Compare and verify the predictions made with the different models with experimental results.

This thesis is structured as follows:

This very first Chapter consists of the introduction, second Chapter presents some multipactor basic concepts and a brief summary of the state of the art in multipactor theory, design and test.

The following two Chapters ( 3 and 4) contain a list of the most relevant publications done in this study. There are a total of five papers. The first two deal with multipactor long-term discharges and a description of the physics of the discharge, including theory and numerical simulations. The next two papers describe thoroughly the multipactor non-stationary theory for single and multi-carrier signals, which is the core of this thesis. Finally the last paper presents an application of the non-stationary theory to a multipactor prediction method. Experimental results are presented and compared successfully with the predictions.

Chapter 5 contains a general discussion about the main findings of the work presented in Chapters 3 and 4. A general review of the whole multipactor theory is presented, ranging from the classical theories, going through the first statistical contributions and ending with the non-stationary theory presented in this thesis. Next, the main prediction methods are also reviewed and compared with the ones presented in this work.

Finally, Chapter 6 presents the most relevant conclusions of the thesis.

Appendices at the end of this book illustrate some interesting and useful topics which are related to this work but outside from the main research line. 


\section{Chapter 2}

\section{The multipactor phenomenon}

The multipactor effect is an electron discharge which takes place between dielectric or metal surfaces in the presence of a high-level and high-frequency electric field. Vacuum conditions, or sufficiently low pressures, are necessary in order to ensure that the electron's mean free path is larger than the separation between impact surfaces ${ }^{1}$. Multipactor was originally discovered and experimentally characterized by [Far34], although current multipactor theory is supported on the works done by [GvE48, HW54, Vau88].

The process can be conceptually described as follows: The electromagnetic fields inside the microwave device exert a force on preexisting free electrons, known as the Lorenz force. These electrons, called primary ones, are accelerated towards the device walls. Under certain field conditions ${ }^{2}$, such electrons may impact on the device surfaces with enough energy in order to generate new secondary electrons according to the material Secondary Electron Emission (SEE) properties. If the impact occurs when the electric field reverses direction, the secondary electrons will be accelerated again towards other metal surfaces, impacting in the same manner as the primary ones and gen-

${ }^{1}$ Such a separation will be referred as gap throughout the text.

${ }^{2}$ Amplitude, frequency and phase. See 5.1.1 
erating more secondary electrons. Therefore, multipactor consists on an electronic bunch moving back and forth in resonance with the electric field which grows impact after impact.

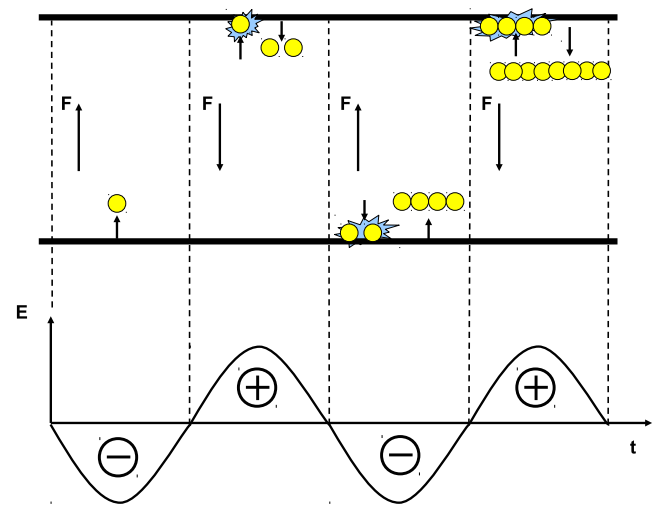

Figure 2.1.

Multipactor description process between the influence of AC field in two surfaces. $\vec{F}$ represents the Lorentz force moving the electrons.

Fig. 2.1 shows a conceptual view of a two-surface multipactor process between two parallel plates when a differential AC voltage is applied on the surfaces. First, a primary electron is accelerated towards the upper plate during the first half cycle of the AC field. The electron strikes the upper surface when the field reverses with enough energy to produce secondary electrons, which are accelerated back to the lower plate. The process repeats creating an electron avalanche.

The electron dynamics of a multipactor discharge can be much more complex than the example above, but in general, it can be classified in two ways: The order of resonance, and the kind of surface interaction. The order of the multipacting resonance is defined as the number of signal half-cycles that an electron takes between interactions with surfaces (See Fig. 2.2). On the other hand, depending on the geometry of the device and the location of the multipactor discharge, the electrons may impact with different surfaces of the device. Typically, multipactor theories cover two kind of interactions: Single-surface multipactor occurs when electrons impact with the same surface of emission, and double-surface multipactor takes place between two facing surfaces. 
The classical multipactor theory proposed by [GvE48, HW54] is based on a one-dimensional and parallel-plate model which studies double-surface interactions. Later on, other works have extended the classical theory to other geometries such as rectangular waveguides $\left[\mathrm{SRA}^{+} 07\right]$, coaxial waveguides $\left[\mathrm{UAL}^{+} 07 \mathrm{~b}\right]$, waveguide irises $\left[\mathrm{UAL}^{+} 07 \mathrm{a}, \mathrm{SRU}^{+} 08\right]$, Helix antennas $\left[\mathrm{RAJ}^{+} 10\right]$ and other signals such as multipactor with modulated signals $\left[\mathrm{SBA}^{+} 10\right]$. Even if these theories give a valuable qualitative description of the multipactor phenomenon in such geometries, they are based in canonical geometries which are usually too simple to represent real circuits and, on the other hand, they are still based on the classical one-dimensional theory, which is known to be accurate only for the first multipactor order region $^{1}$.

Therefore, in practical situations, the multipactor prediction techniques are based on the parallel-plate model [EST03], since, on the one hand, it is one-dimensional and therefore simpler to develop and, on the other hand, it is accepted to represent the worst-case for double-surface multipactor. This means that for similar gap, frequency of operation and material, the parallel-plate geometry will yield the lowest breakdown voltage as compared with other geometries. Or, in other words, any deviation from the parallel plate geometry and uniform field distribution will produce some kind of extra electron loss mechanism that will increase the breakdown voltage.

Thus, multipactor predictions with parallel-plate geometries always have a certain margin with respect to the real geometry and, therefore, guarantee that the resulting breakdown power will be conservative. Unfortunately, when the real geometry differs considerably form the parallel-plate case, such a high margin may imply unaffordable design constraints. For those cases, numerical multipactor prediction may provide more accurate breakdown predictions, and therefore lower margins. Using numerical simulators, multipactor has been investigated also in circular waveguides $\left[\mathrm{PBG}^{+} 09, \mathrm{SZA}^{+} 10\right]$, coaxial waveguides $\left[\mathrm{PTV}^{+} 06, \mathrm{PTV}^{+} 09, \mathrm{SZU}^{+} 07\right]$ wedge waveguides $\left[\mathrm{HRS}^{+} 11, \mathrm{SRZ}^{+}\right.$08], microstrip lines $\left[\mathrm{SAM}^{+} 09, \mathrm{SRS}^{+}\right.$09], and ridge and multi-ridge waveguides [GISA ${ }^{+} 12$ ]. However, these studies are limited to isolated waveguides in which the RF fields are not representative of such appearing in real and much more complicated RF structures. Thus, for accurately analyzing multipactor in real mi-

${ }^{1}$ for higher orders this theory yields conservative predictions. See Section 5.1.3 
crowave circuits, commercial electromagnetic software tools are the most reliable option among all [FES, SPA, CST]. Such tools compute the real field distribution and solve electron trajectories inside the complex geometry.
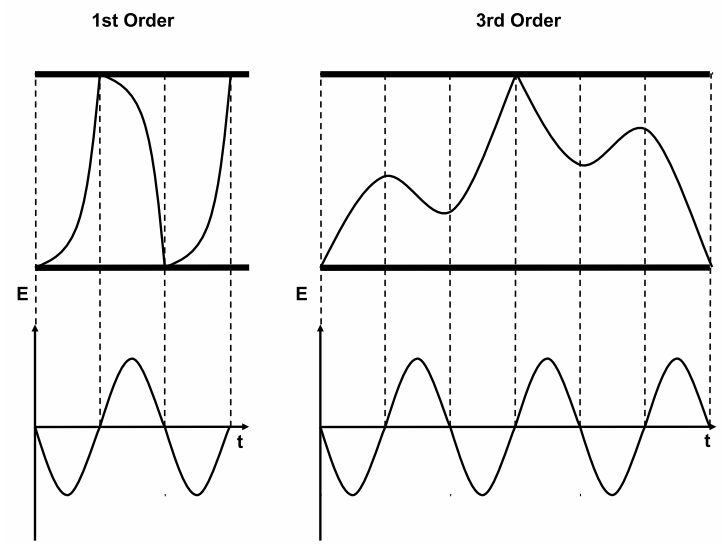

Figure 2.2.

Visualization of $1^{\text {st }}$ (left) and $3^{\text {rd }}$ (right) two-surface multipactor orders: position of the electron and driving electric field as a function of time.
${ }^{1}$ Multipactor may take place in many different kind of surfaces, including metals and dielectrics

With respect to single-surface multipactor, it is less frequent and is typically associated with the presence of dielectrics ${ }^{1}$. Multipactor in dielectrics has the peculiarity that the dielectric surface gains electric charge when electrons are released, generating a DC field which pulls the electrons back to the emission surface $\left[\mathrm{TCB}^{+} 05, \mathrm{TCV}^{+} 06\right.$, $\left.\mathrm{TPCM}^{+} 10\right]$. In the case that the RF field is parallel to the surface, such as in dielectric windows, the RF electric field accelerates the electrons parallel to the surface while the DC field attracts the electrons to it. As a result of this, the electrons impact the surface at high speed and high angle of incidence, thus releasing extra electrons by secondary emission effect and producing a single-surface discharge. Fig. 2.3 shows this particular case extracted from $\left[\mathrm{KLA}^{+} 98\right]$ where the electron trajectory under such circumstances is shown. In these cases the electron motion does not need to be in resonance with the RF field in order to achieve multipactor $\left[\mathrm{KLA}^{+} 98\right]$. This fact, together with the poor thermal dissipation of dielectrics compared with metals, makes multipactor phenomenon be potentially more dangerous and destructive in dielectrics than in metals.

Although the above theories make an attempt to predict the nec- 


\section{Figure 2.3.}

Single surface multipactor on a dielectric parallel to the RF field. The direction of the DC and $\mathrm{RF}$ fields as well as the electron trajectory are shown. Large angle of incidence at electron impact, $\xi$, can be observed.

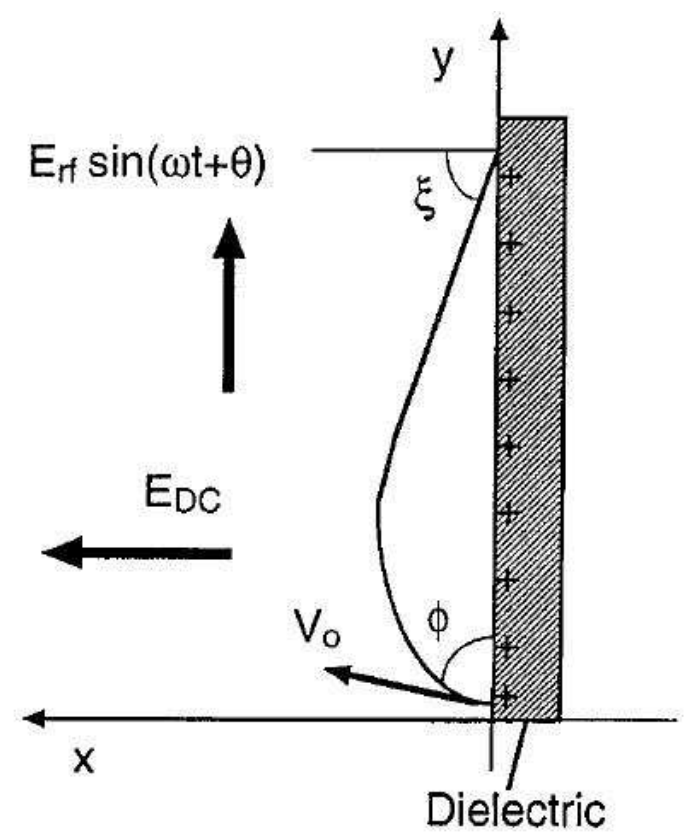

essary conditions for multipactor to take place, there is another important factor to take into account: There must be an initial population of electrons inside the component in order to set-up a discharge. A single initial electron does not ensure a discharge, since its position, energy and emission phase (with respect the RF field) may not produce secondary electrons on impact. Therefore, the higher the number of seeding electrons the higher probability of a multipactor discharge to take place.

There is always some contribution coming from the electrons released by field emission from the surfaces of the device. In space environment the main source of seeding electrons is the particle radiation due to the trapped environment in the magnetosphere, Solar Flares or Cosmic Rays. The total flux of electrons reaching the interior of the device depends on the satellite materials and orbit conditions.

On the other hand, multipactor tests done in Earth's laboratories need some kind of artificial electron seeding in order to obtain significant results in a reasonable amount of time [WJ89, GW96, RW95]. 
There are two kind of popular seeding techniques for multipactor testing, radioactive sources and Ultra-Violet (UV) lamps, which are reviewed in Appendix A.

\subsection{Secondary Electron Emission effect}

As introduced before, Multipactor discharges are produced by Secondary Electron Emission (SEE) from the component surfaces, thus, the study of multipactor is strongly related with the study of SEE phenomenon. The Secondary Electron Emission (SEE) phenomenon was discovered more than one hundred years ago and since then it has been intensively investigated (see for example [Bru54, LD57, Ste57, CE74, Vau89, FP02]). Basically, when an electron hits a material, it interacts electrically with the secondary electrons belonging to the outer shells of the atoms in the structure. Depending on the impact energy and angle, the primary electron can be totally deflected (specular reflection); can enter inside the material, loose some energy, and then being deflected backwards (inelastical backscattering); or it can penetrate in the material and transfer part of its energy to secondary electrons which are released backwards from the material surface (true secondary emission). In summary, any of the three following phenomena can happen depending on the impacting energy and angle: the electron is elastically or inelastically backscattered, new electrons are released by SEE effect, or it can be simply absorbed (see Fig. 2.4).

The dynamics of each kind of phenomena are different, as well as their participation in a multipactor discharge. Whereas the elastically backscattered electrons are reflected with an energy and departure angle equal to those of the impact, inelastically backscattered ones lose some energy when colliding. On the contrary, the emission energies and angles of secondary electrons are completely independent of, and generally much lower than, the electron impact conditions (provided that the impact energy is high enough to produce secondaries).

Being the SEE a random process, the Secondary Electron Emission Coeficcient (SEEC), also referred as Secondary Emission Yield (SEY), is the ratio between emitted and impacting electrons on a material surface, being a characteristic of each material. The total 


\section{Figure 2.4.}

Schematic of the three different kind of electron interaction with matter.

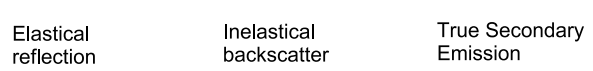
Primary electron

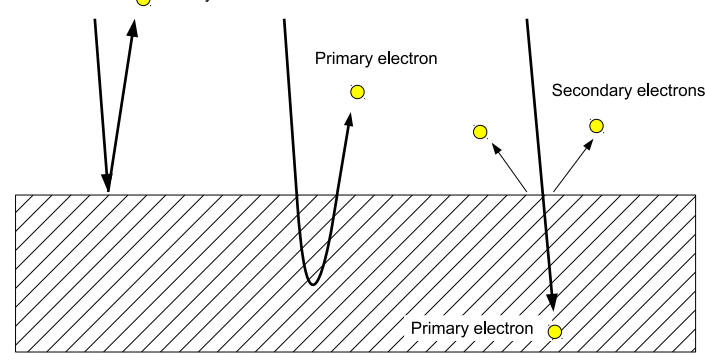

SEY usually adds the contribution of all the previous kinds of electron emission explained above. These phenomena exhibit a random nature and, therefore, the SEY must be understood as an average figure. SEY larger than one means that extra electrons are generated for each impacting electron (in average), while for SEY lower than one, a fraction of the impacting electrons is absorbed by the material (totally if it is zero).

In spite of its name, the SEY accounts not only for true secondary electrons, but it also includes backscattered ones. The reason for this is that is not easy to distinguish both types in SEY measurements.

The SEY value varies with the impact energy and angle of incidence of the primary electron. Although the SEY curves differ for each material, they have a common shape. A typical SEY curve is represented in Fig. 2.5, extracted from $\left[\mathrm{dLPA}^{+} 06\right]$, where the SEY (Total SEY in the figure) has been theoretically decomposed in its different components:

- At low impact energies, the SEY is dominated by elastically backscattered electrons, with a value of SEY close to 1, which implies that almost all low energy electrons are reflected at the material surface $\left[\mathrm{CCF}^{+} 04\right]$.

- As the energy increases there is an absorption process, i.e. SEY is lower than 1 . 
Figure 2.5.

Typical

SEY

curve

extracted

from $\left[\mathrm{dLPA}^{+} 06\right]$. Total

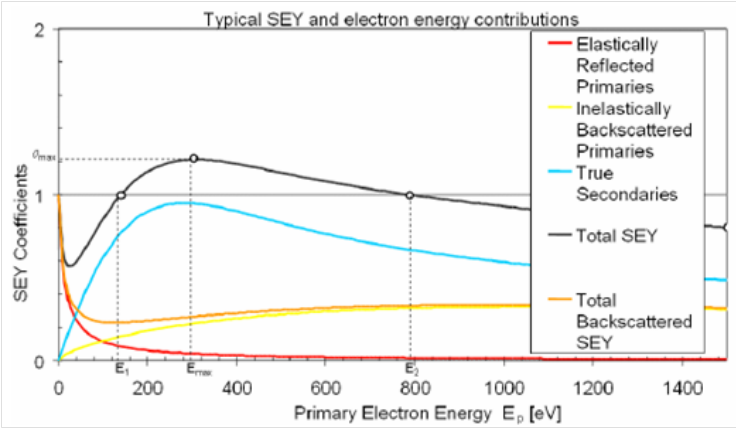

SEY is decomposed in true secondaries, elastically and inelastically backscattered electrons curves. Maximum SEY $\sigma_{\max }$ at $W_{\max }$ and first and second crossover points, $W_{1}$ and $W_{2}$ for which the SEY equals one, are also marked. The value of the SEY at low energies approaching one is due to the high reflectivity of low energy electrons.

- For higher energies true secondary electrons dominate the SEY. The secondary emission theory assumes that as the electron penetrates and slows down in the material, all the dissipated energy is employed in generating secondary electrons [LD57, Ste57]. The rate of energy lost by the primary is governed by the stopping power, which is known to be energy dependant in a way that there is an energy of the primary electron, $W_{\max }$, for which the stopping power is maximized and thus the SEY, $\sigma_{\max }$. For energies above and below such a value the SEY decreases towards 0 . if $\sigma_{\max }>1$ there are two values of the primary energy (labeled as $W_{1}$ and $W_{2}$ )for which the SEY is equal to 1 .These are called first and second crossover points, respectively.

- For energies higher than $W_{2}$ the SEY is dominated by both true secondaries and inelastically backscattered electrons. It is always lower than 1 .

Equivalently, the SEY may be given as a function of impact velocities instead of kinetic energies. There is also a dependence of the SEY 
on the angle of incidence, where zero angle stands for perpendicular impact. As the impact angle increases the whole SEY curve changes increasing the SEY peak and the second crossover point $\left(W_{2}\right)$. As an example, Fig. 2.6 (extracted from [Vau89]) shows two SEY curves for a typical material for different values of angle of incidence. The curves have been drawn with Vaughan's SEY equation which does not consider elastically backscattered low energy electrons. However, the curves illustrate the effect of the angle of incidence at higher energies, showing that oblique impacts $\left(60^{\circ}\right.$ in the graphic) present higher SEY maximum value and second crossover point than the normal incidence curve.

For a given material, the SEY is also very sensitive to the surface conditions, such as roughness, presence of impurities or even fingerprints. To reduce the SEY to the minimum, the materials must be scrubbed, cleaned and handled with caution.

As mentioned before, a necessary condition for multipactor to occur is that the SEY must be equal or greater to 1 and therefore, the impact energy of primary electrons must be comprised between the first and second crossover points of the SEY curve for a given incidence angle $\left(W_{1}\right.$ and $\left.W_{2}\right)$. Furthermore, the rate of growth of the electron population increases exponentially with the SEY value. Therefore, the choice of a material with low values of SEY and high $W_{1}$ may help to mitigate partially or totally the multipactor effect.

\section{Figure 2.6.}

Vaughan's approximation to SEY curves. Low energy high reflective electrons are not modelled. Dependence of the SEY on angle of incidence is observed.

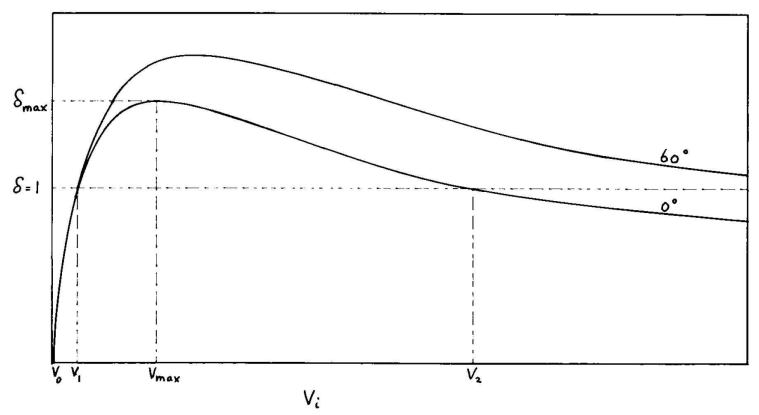

It becomes evident, then, that in order to model and predict properly multipactor it is crucial to have an accurate model of SEE fitting the experimental results for the materials to be employed in the 


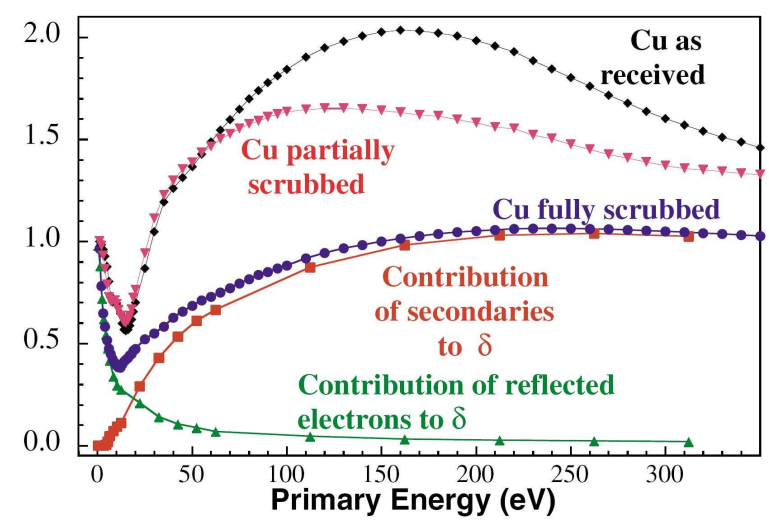

Figure 2.7.

Experimental SEY data for copper. Extracted from $\left[\mathrm{CCF}^{+} 04\right]$.

construction of the device. Vaughan's proposes in [Vau89] a parametric formula of SEY which admits $\sigma_{\max }$ and $W_{\max }$ as input parameters and allows fitting experimental data. Fig. 2.6 shows an example of Vaughan's model with the effect of different impact angles. Vaughan's formula fits reasonably well the experimental data and has been widely used for modelling multipactor. However, it does not differentiate between backscattered electrons and secondary ones, constituting a source of error since they have different dynamics. Moreover, it does not take into account the existence of low energy reflected electrons. Even if those do not directly participate in the multipactor process, they are long-lived inside the device due to its high reflectivity, thus contributing to accumulation effects between consecutive multipactor discharges. This is of particular interest in multicarrier operation, as described in chapters 4.4 and 5.2.

The Furman and Pivi SEY statistical model [FP02] takes into account the different nature of the electrons contributing to SEY, and the resulting curves are very similar to Fig. 2.5, but when fitted to experimental data less importance to low energy electrons is given, resulting in a SEY tending to 0.5 at low energies instead of 1 , which means that only half of the low energy electrons are reflected and the other half absorbed. Recent SEY measurements undertaken by Cimino et al. $\left[\mathrm{CCF}^{+} 04\right]$ suggest that Furman and Pivi model underestimates the importance of low energy electrons, due mainly to the lack of resolution in the experimental measurements of SEY at low 
energies. Cimino et al. conclude that the SEY for most materials tends to one at low energies, i.e. most of the low energy electrons are reflected. Experimental results from $\left[\mathrm{CCF}^{+} 04\right]$ are shown in Fig. 2.7. Inelastically backscattered electrons are not differentiated from secondaries. The curves follow the same behaviour as Lara et al. (see model of Fig. 2.5). The decrease of SEY when scrubbing the cupper is also observed.

These results are of the highest importance for multipactor in multicarrier operation due to the aforementioned accumulation effects introduced by high reflective low energy electrons. As a conclusion, models like Lara et al. $\left[\mathrm{dLPA}^{+} 06\right]$ are the most suitable ones for multipactor modelling purposes.

There are no universal SEY curves for a specific material. The study done in [AUR11] demonstrates that the particular manufacturing or coating process done by each company affects the SEY curve of a material. In other words, two silver coatings, for example, done by different companies ${ }^{1}$, may have a different SEY curve. Because of that, it is also very important for multipactor prediction to have particular SEY measurements of the device which is going to be tested. Another problem, also reported in [AUR11] is the SEY Ageing, which consist in a degradation of the surface due to environment exposure and oxidation that increases overally the SEY curve, also lowering the $W_{1}$ energy. This produces lower multipactor thresholds. In [AUR11] it has been observed that this Ageing process degrades the material progressively reaching a stability after 6 months (in average). According to [AUR11], the impact on the multipactor threshold depends on the material but has typical values of $3 \mathrm{~dB}$ and maximum values up to $7 \mathrm{~dB}$. This impact should be considered also in prediction as an additional margin.

A very important aspect of secondary electrons but that has been historically ignored in classical multipactor theories is the dynamics of the secondary emitted electrons, i.e. angle and velocity of emission. Classical multipactor theories consider erroneously a fixed and deterministic emission velocity of secondary electrons, when actually it is a random quantity independent of the primary impact energy. The emission angle is also governed by a random process which also affects the multipactor susceptibility regions [VSSS06]. 


\subsection{Saturation}

The definition of the multipactor effect given so far, and the derivation of the analytical equations of the classic theory describe a picture where, when a multipactor discharge takes place, the electron population increases indefinitely. This is, of course, an unnatural behavior and it is due to the fact that the electric field created by the electrons has not been taken into account, which is responsible for the multipactor saturation. Saturation may be caused by two main effects: space charge and detuning.

Space charge is due to the culombian repulsion among existing electrons. The electric field that the electron cloud creates in the device volume, alters the original field that started multipactor and may break the resonance conditions. The space charge is also responsible for the main consequences of multipactor: noise generation and power losses.

According to Vaughan [Vau88] first, and Riyopoulos [Riy97] later, when the electron population is low enough to neglect space charge, and multipactor order is low, all the electrons move in-phase, due to the phase focusing effect, forming a thin sheet moving from plate to plate. As the population grows, the mutual culombian repulsion between the electrons make the sheet broaden. In an initial stage, the phase error induced in the electrons by the broadening may be compensated by the phase focusing effect. But after reaching the saturation level, the error cannot be compensated and new electrons are lost, reaching the steady-state population.

In [Riy97], Riyopoulos presents a multipactor theory and the results of a multipactor PIC simulation accounting for saturation effects. Fig. 2.8 shows, to the left, the electrons forming a thin sheet when there is not space charge yet, and, to the right, the electron dispersion when the saturation level is reached. A typical electron population evolution with time, also extracted from [Riy97], is presented in Fig. 2.9, where the saturation effect can be observed.

Other saturation mechanism is the detuning, which occurs in high Q cavities (very narrow bandwidth), where the induced current created by the electron sheet going back and forth loads the system and detunes it, thus changing the response of the system, and loosing the resonance conditions of the cavity $\left[\mathrm{KLA}^{+} 98, \mathrm{KL} 95\right]$. 


\section{Figure 2.8.}

(Left) electron sheet when the space-charge effect is not important. (Right) electron sheet after some RF cycles. The electron population has grown and the sheet has broaden (steady-state has been reached). The graphs are the result of a PIC simulation presented in [Riy97]. The plates are disposed along $x$-axis separated across $y$-axis.

\section{Figure 2.9.}

Example of a typical electron growth for a silver coated rectangular waveguide of dimensions $a=22.86 \mathrm{~mm}$, $b=0.1 \mathrm{~mm}$ and a frequency of $f=10 \mathrm{GHz}$. After some cycles, the discharge saturates and the electron population stays around a stable value. The saturation level is also dependent on the applied RF voltage.
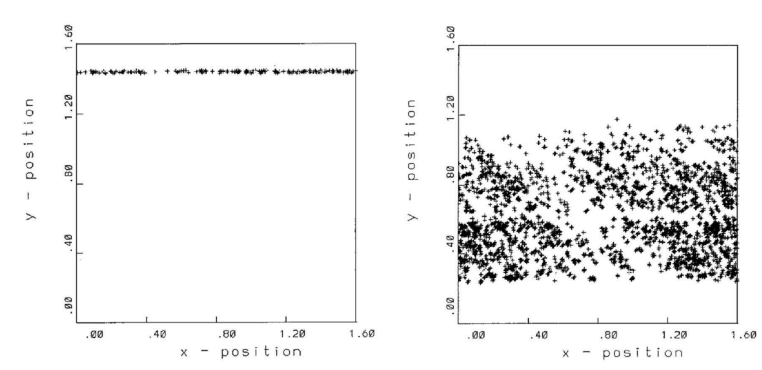

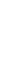

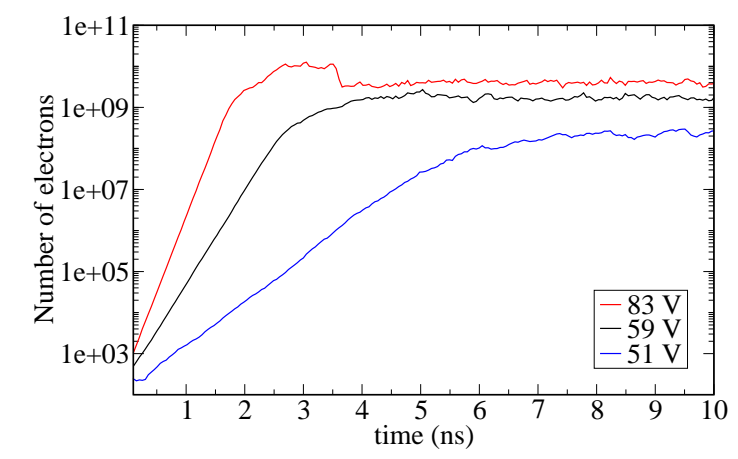




\subsection{Single-carrier and multi-carrier signals}

${ }^{1}$ The resulting envelope is similar to an AM modulation

${ }^{2}$ The rate of growth mainly depends on the SEY and multipactor order

A typical multi-carrier signal is composed of several modulated carriers with small frequency separation. As a result, the signal experiences periods of constructive and destructive addition of the different carriers, resulting in a time-varying periodic envelope of the signal ${ }^{1}$. Such a situation adds a dynamical behavior to the multipactor process, which is not well understood yet. When the amplitude of the envelope is above the $\mathrm{CW}$ breakdown level, the multipactor resonance conditions are met and there is electron multiplication. When it is below that level, resonance is broken and electrons are absorbed.

A multipactor discharge is said to occur when the electron population has grown beyond a certain level which produces detectable effects in laboratory. The single carrier case is easy to characterize because when multipactor conditions are accomplished, the electronic bunch grows in a exponential manner. Therefore, no matter the rate of growth, the electron population will reach the saturation level sooner or later ${ }^{2}$.

On the contrary, the amplitude of a multicarrier signal is not constant. The addition of carriers with different frequencies result in a time varying envelope whose shape depends on the relative phase shift among the carriers (see Fig. 2.10). Therefore, a multipactor discharge in this kind of signals can be understood as periodic electron avalanches, which take place whenever the signal envelope complies with resonance and multipactor conditions ("on" intervals), with alternated periods of inactivity when the amplitude is too low to hold a discharge ("off" intervals").

Despite of the absorption process in the "off" periods, it is possible that some fraction of electrons survive from peak to peak of the signal's envelope, creating an inter-period accumulation process. Hence, even if enough electron density is not reached in a single peak to produce a discharge (a single-event discharge), the population can grow from peak to peak indefinitely to its saturation value in what is called a long-term discharge. The theoretical development and numerical simulations for parallel plates presented in $\left[\mathrm{AVG}^{+} 07\right]$ may illustrate this issue. Fig. 2.11 shows an example of a long-term discharge for a 6-carrier signal and a frequency separation of $100 \mathrm{MHz}$.

In order to properly characterize the accumulation effects it be- 
Figure 2.10.

Example of a 5 carrier in-phase composite signal centered at 2.86 $\mathrm{GHz}$ and with equal frequency separation of 330 $\mathrm{MHz}$

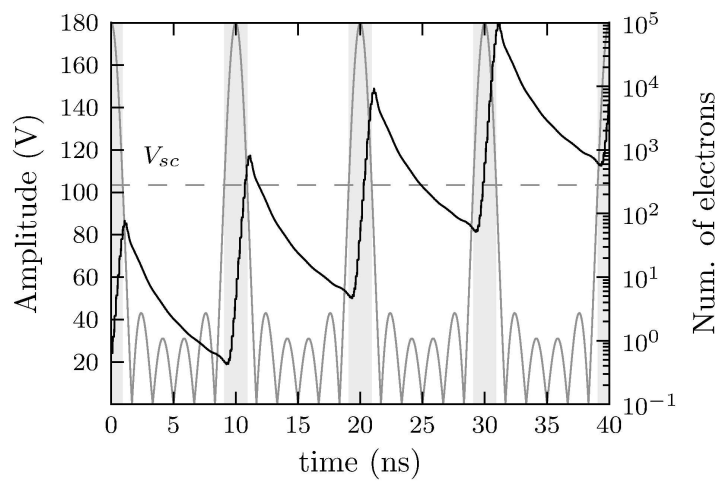

Figure 2.11.

Evolution of electron population versus time for a for a 6-carrier signal and a frequency separation of $100 \mathrm{MHz}$.

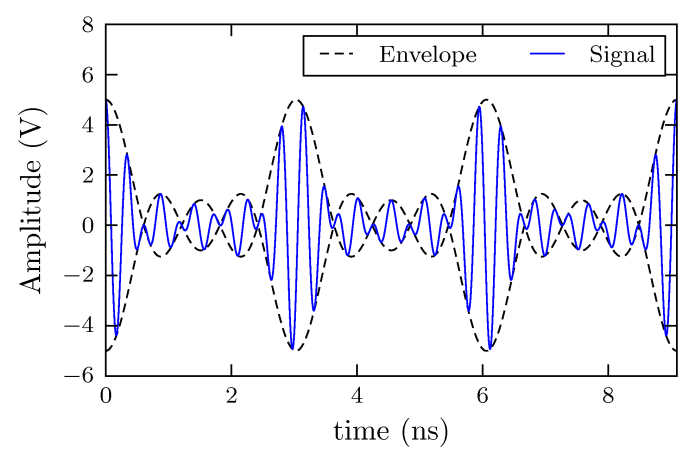

comes necessary to model the absorption processes which rely basically in the loss of resonance and the lower-than-one secondary emission coefficient during "off" periods. Elastically reflected electrons at low energies play an important role since they may persist "bouncing" between the device walls even if resonance conditions are lost, thus highly contributing to the accumulation effect. This accumulation effect is more probable to occur working in high $f \times d$, since the electron transition time between plates is larger. 


\section{$2.4 \quad$ State of the art}

\subsubsection{Necessary conditions}

Basically, and summarizing the above sections, Multipactor depends on the following parameters:

Vacuum condition: Multipactor occurs only at low pressures $(<$ $10^{-5}$ mbar). At this pressure, the mean free path of electrons is in the range of centimeter, while the equipment typical gap size is in the millimeter range.

Frequency of operation and geometry: The amplitude of the electric field, the frequency and the gap size must be those allowing the electron motion to be in resonance with the RF field, in order to produce an electron avalanche. In general higher frequencies and/or gap sizes imply higher breakdown levels.

Secondary Emission Yield: The Secondary Emission Yield (SEY), also called Secondary Electron Emission Coeficcient (SEEC), is a material characteristic that relates the number of secondary emitted electrons with the number of primary impacting electrons. A condition for multipactor to occur is that the average SEY must be larger than 1 . This value depends on the primary electron impact energy and the angle of incidence as explained in the next section. Experimental measurements show that contaminants such as light, fingerprints, dust, adhesives and lubricants reduce the threshold for multipactor effect about -3 $\mathrm{dB}$ on average.

\subsubsection{Techniques against Multipactor}

In order to avoid multipactor it is necessary to, at least, break one of the necessary conditions for the discharge. The most obvious technique is done in the component design phase and simply consists in trying to increase the size of the gap between opposite plates in the multipactor critical gap, thus increasing the multipactor RF breakdown level.

If the multipactor breakdown level cannot be increased enough during the design phase, there are other techniques that may help 
to mitigate multipactor. However, these imply extra size, weight and cost of the device. The different strategies can be classified as follows:

Change the shape of the RF structure: There are special geometries that are designed to increase the multipactor breakdown power, such as the wedge waveguide $\left[\mathrm{Cho00}, \mathrm{HRS}^{+} 11\right]$ or quasiperiodic continuous profile structures $\left[\mathrm{AAA}^{+} 09\right]$.

Changing the properties of surface conditions: The SEY properties of each material is different. Therefore, in order to reduce the SEY, one technique consists in covering the surface with coatings of materials with a known lower secondary emission coefficient, such as Ti, TiN. On the other hand, surfaces that cannot be coated can be cleaned with different chemicals to reduce secondary emission significantly. Other techniques consist on applying a magnetostatic field pattern on the metal surface $\left[\mathrm{CMM}^{+} 09\right]$.

Avoid vacuum conditions: By either foaming or pressurizing the complete device or the critical gap, vacuum is broken and the electrons do not move freely anymore, thus completely avoiding multipactor. However, when pressurizing the device there is a high risk of Corona discharge.

DC Biasing: It consists on the superposition of electric DC-fields over the resonant RF-fields to obtain a disruption of the resonance conditions.

Transverse magnetic fields: If transversal magnetic fields are present, the electrons are accelerated perpendicularly to their direction of motion, which may increase the impact angle of incidence. As detailed in next section, the latter implies a higher Secondary Emission Yield. In these cases, the presence of low magnetic fields may lower the multipactor threshold $\left[\mathrm{GPB}^{+} 03\right.$, $\left.\mathrm{GIPA}^{+} 12\right]$. However, higher level magnetic fields may deviate completely the trajectory of the electrons avoiding resonance or even making them return to the emission surface [RCD95]. In this case multipactor is prevented $\left[\mathrm{GPB}^{+} 03, \mathrm{GIPA}^{+} 12\right]$. 
Slot on Broad Walls: It consists of opening a slot on the broad wall along the center plane of the rectangular waveguide $\left[\mathrm{GPB}^{+} 03\right]$. The slot is narrow enough so that no RF field is leaking into it. This concept works by letting electrons drift in a field free region and hence provides a perturbation resonance. This method reduces the rate of growth of the electrons in a multipactor discharge which is not of high importance in single carrier operations, since sooner or later multipactor will take place. However, it becomes important in multicarrier operation, since multipactor discharges are short and consecutive. Therefore, the rate of growth may set the conditions for multipactor to start.

Besides those involving foaming or increasing the pressure, these techniques do not completely mitigate the multipactor discharge. They only increase the breakdown power. Therefore, even if they are applied, multipactor prediction methods are necessary in order to establish the new RF breakdown threshold.

\subsubsection{Multipactor design and test}

From the space industry point of view, the multipactor phenomenon appears typically in microwave devices or guides working at low pressures or vacuum such as satellite communications devices. As stated before, the effects of the multipacting avalanche ranges from the degradation of the signal and heating to the damage or even destruction of the device. For these reasons, multipactor is considered as a critical issue in the design of microwave devices for space applications by the main Space Agencies, like NASA and ESA. Hence, the industry makes a big effort in the design phase in order to avoid it, selecting the appropriate materials, the device geometry and testing the devices in laboratory to assess the maximum RF power they can handle before multipactor appears.

\subsubsection{Multipactor prediction}

Specifically, multipactor in single carrier case is a well known phenomenon which has been studied during the last fifty years, being its theory well developed and supported by diverse experimental results. 
Multipactor equations for the parallel plate case, relating the different parameters involved, have analytical closed expressions, which can be used to map the multipactor regions (combinations of parameters which start multipactor) in $V$ versus $f \times d$ plots, where $V$ is the equivalent voltage across the gap, $f$ is the carrier frequency and $d$ is the gap. These are called the multipactor susceptibility curves, which stand for different materials, and are taken as standard for the multipactor design margins by ESA [WJ89].

But, unfortunately, since these curves can be applied to real microwave components only when the gap size is relatively small compared with the rest of dimensions, the lack of reliable susceptibility curves for all geometries renders the design threshold hard to be established. Moreover, after being manufactured, storage conditions, manipulation, impurities and other effects can lower the multipactor threshold ${ }^{1}$. For such reasons, ESA specifications for multipactor make the devices operate at least $6 \mathrm{~dB}$ below the susceptibility curves' margin [WJ89], and impose the devices to be tested in a laboratory. These tests are very costly and, in addition to the fact that current thresholds are overestimated with the consequent oversizing of the components, derives in higher costs of manufacturing and higher mass budgets, which are a critical issue for space missions. There currently exist some multipactor prediction CAD software tools for the design of microwave components, usually combining different electromagnetic numerical solving methods with multipactor prediction modules, which may handle complicated structures [FES, CST, $\mathrm{AVR}^{+} 08$ ].

In practical applications, a multipactor discharge occurs when it has measurable consequences (noise, harmonics, reflected power, detuning, etc.). It is obvious that there must be a limit electron density in a discharge beyond which multipactor is said to occur. This is called the multipactor criterion. Currently, there is not any theoretical study that determines such criterion. For both single-carrier and multi-carrier long-term discharges this is not of great importance since, once resonance conditions are met, the electronic density will grow indefinitely until saturation. But this is not the case for multi-carrier single-event discharges since the multipactor population reaches a certain peak value during the "on" interval. A multipactor criterion is needed to establish if such an electron peak is enough to create a measurable multipactor discharge . 
Currently, the multipactor prediction methods used in the component design in multi-carrier operation only consider single-event discharges. The single-carrier theory is applied with little modifications and its susceptibility curves with the same margins are applied. However, there is not a general agreement on the multipactor criterion to apply. The most restrictive one is the peak power or the $K^{2}$ rule, which forces the in-phase maximum peak power, $K^{2} P_{M C}$ to be below the single-carrier breakdown level $P_{S C}$, where $K$ is the number of carriers and $P_{M C}$ is the individual carrier power $(S C$ and $M C$ stand for single-carrier and multi-carrier, respectively). This rule does not allow the multi-carrier envelope to be above the threshold at all, and therefore no electron production exists.

Internal research in ESA produced the 20-gap-crossing rule $\left[\mathrm{MMS}^{+} 97\right]$ which proposes a more relaxed criterion: "Multipactor will occur only if the envelope is above the single-carrier threshold a time equivalent to 20 electron gap crossings". This rule throws more relaxed predictions compared to the $K^{2}$ one. However, this result has no physical basis and came up from numerical simulations and experimental results on a limited number of samples and multi-carrier signals. In addition, this rule does not take into account many critical parameters in multipactor such as the shape and amplitude of the envelope (the rule says higher than the threshold but not how much higher) and the SEY properties of the material.

In spite of this, the 20-gap-crossing rule (or similar) has been widely accepted by the industry because it is thought to be more relaxed than the $K^{2}$ one, but still conservative (due in part to the large design margins applied to the predictions). Nevertheless, it is far from being an standard because each manufacturer employs a different rule according to their know-how or the customer requirements. As a consequence there exist many rules such as $N^{2}, N, 20$-gap-crossing rule, 10-gap-crossing rule, the 4-gap-crossing rule, etc.

A good prediction method should, firstly, incorporate also longterm discharges, and secondly, use a reliable multipactor criterion for the single-event ones. Recently, some new theories have linked the multipactor discharge with its radiation spectrum $\left[\mathrm{SAG}^{+} 08, \mathrm{GSA}^{+} 09\right.$, $\left.\mathrm{JGME}^{+} 10, \mathrm{JMR}^{+} 12\right]$. With these theories it is possible to predict not only if there will be resonance conditions but also what will be the effects on the circuit in terms of noise and harmonics. Therefore, 
these theories could be used to establish a more reliable multipactor criterion.

Summarizing, there is a lack of a reliable and standard multipactor prediction method for multi-carrier signals. A new and accurate prediction method is necessary in order to, first, have good understanding and control over the multipactor discharge, second, have a standard criterion in the industry, and third, have more accurate predictions. All this will help to reduce test and design margins, with the subsequent cost savings.

\subsubsection{Multipactor test}

Multipactor may happen in different technologies and frequency bands. However, their respective set-up are very similar. The typical one consists of (see Fig. 2.12):

- The Device Under Test (DUT). It is the sample on which multipactor is going to be tested.

- A vacuum chamber. Where the DUT is placed. It provides a vacuum environment to ensure that the electrons move freely and multipactor is possible. If the DUT is closed (for example in waveguide technologies) venting holes must be present to ensure a complete de-pressurization inside the component. Otherwise, corona discharges could take place and distort the experiment. The same applies to other waveguide sections of the set-up which are inside the vacuum chamber.

- Signal Generators / power amplifiers. To produce an RF signal at variable output power to feed the system. The signal can be Continuous Wave (CW) or pulsed with some specified duty cycle (typically of 2-5\%). Multipactor tests usually take long operation times and CW signals are not usually employed due to overheating of the amplifiers. Typical tests use un-modulated $\mathrm{RF}$ signals. In the case of multi-carrier testing, diverse amplifiers at different frequencies may be used and the resulting signals are combined with a multiplexer in a single DUT.

- Detection methods. There are two kind of them, local and global ones. Local methods look for local evidences of a multi- 
pactor discharge, such as an increase in the electron density inside the DUT, or the production of photons due to local Corona discharges produced by outgassing. Global methods control signal parameters at input and output of the DUT, through spectrum analyzers or oscilloscopes to detect signal variations due to a multipactor discharge. The correct set-up and accuracy of the detection methods is crucial for the multipactor test.

- Electron Sources. Produce an external source of electrons to ensure enough initial population to produce a discharge in the laboratory typical test times (ranging from minutes to hours). Appendix A summarizes the most popular electron seeding techniques.

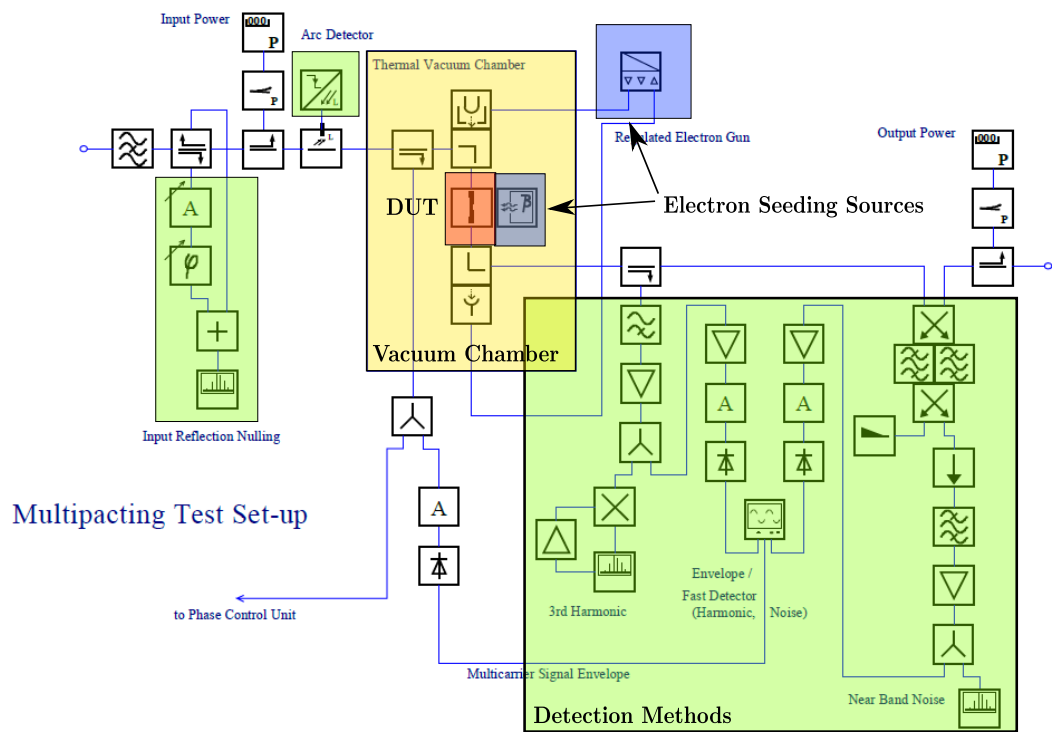

Figure 2.12. Schematic for typical multipactor test set-up.

There are two main issues when testing multipactor. The first one is to ensure that a multipactor discharge will take place (if the necessary conditions are met). In addition, the discharge must be always located in the Device Under Test (DUT). In other words, the 
Figure 2.13.

Ku-band test set-up: Gap sample (a), radioactive source (b), electron gun (c) and coupler for detection of the phase and envelope (d) installed inside the thermal vacuum chamber.

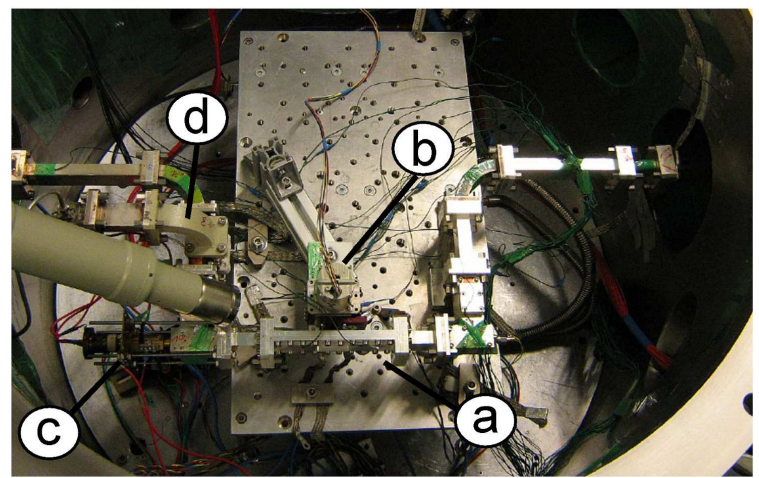

set-up must be multipactor-free. Multipactor has a random nature and strongly depends on the initial free electrons or seeding electrons. In normal operation there is a low number of seeding electrons and the multipactor discharge could appear after months or even years of operation, depending on the space environment of a particular satellite. Since the testing time is limited to few minutes or hours, it is necessary to increase the probability of having the multipactor discharge by increasing drastically the number of seeding electrons. This is done by means of electron seeding sources which provide an extra source of seeding electrons inside the DUT. Seeding sources is a must, specially in pulsed multipactor tests ${ }^{1}$, because, without them, it is very unlikely that multipactor discharges are initiated even if the DUT is prone to them. This would lead to a false non-detection and the device could fail in operation.

The second main issue when testing multipactor is to be able to detect the discharge once it has started. The sensitivity of the detection methods is very dependent on the set-up and manual installation by laboratory operators. This is the reason why it is advisable first to employ more than one detection method at a time and second to run a multipactor test with a well-characterized DUT for each new set-up in order to check that the set-up and detectors are working properly. 


\section{Chapter 3}

\section{Article index}

This chapter lists the articles that are included in this work. Chapter

4 contains the full published version.

\subsection{Long-term multipactor discharge in multicarrier systems (See Section 4.1)}

\subsubsection{Bibliographic record}

- Authors: S. Anza, C. Vicente, B. Gimeno, V. E. Boria, and J. Armendariz.

- Publication: Physics of Plasmas, American Institute of Physics, vol. 14.

- Year: 2007.

- Pages: 082112, 8 pages.

- Keywords: discharges (electric); microwave switches; rectangular waveguides; secondary electron emission. 


\subsubsection{Abstract}

A new mechanism of long-term multipactor in multicarrier systems is studied employing both analytical and numerical methods. In particular, the investigation is focused on the impact that a realistic secondary emission yield at low energies produce on the development of long term multipactor. A novel analytical model for this inter-period charge accumulation is presented using the traditional multipactor theory for parallel plates, and approximating the multicarrier signal as a single carrier signal modulated by a pulsed signal envelope. The analytical predictions are verified by numerical simulations for a typical rectangular waveguide. The analytical and numerical results demonstrate that the susceptibility of the system to develop a longterm multipactor discharge increases with higher values of low-energy secondary emission yield.

\subsection{RF breakdown prediction for microwave passive components in multi-carrier operation (See Section 4.2)}

\subsubsection{Bibliographic record}

- Authors: S. Anza, M. Mattes, J. Armendariz, J. Gil, C. Vicente, B. Gimeno, V.E. Boria and D. Raboso.

- Publication: (Book) Ultra-Wideband, Short Pulse Electromagnetics 9, Part 6, Springer New York.

- Year: 2006.

- Pages: 375-381.

- Keywords: Physics and Astronomy.

\subsubsection{Abstract}

This work addresses the multipactor problem for multi-carrier operation inside rectangular waveguide-based devices, by means of numerical simulations of the electron trajectories and multiplication inside the structure. Accurate field calculation and considering space 
charge effects are mandatory. For this, a PIC-FDTD method has been employed. As a result, novel software has been implemented offering the possibility of predicting multipactor in multi-carrier systems for a wide variety of situations. Simulation results show that multipactor can occur even for short pulse durations if an inter-period charge accumulation is present.

\subsection{Non-stationary Statistical Theory for Multipactor (See Section 4.3)}

\subsubsection{Bibliographic record}

- Authors: S. Anza and C. Vicente and J. Gil and V. E. Boria and B. Gimeno and D. Raboso.

- Publication: Physics of Plasmas, American Institute of Physics, vol. 17.

- Year: 2010.

- Pages: 062110, 11 pages.

- Keywords: electron avalanches; plasma theory; plasma-wall interactions; statistical analysis.

\subsubsection{Abstract}

This work presents a new and general approach to the real dynamics of the multipactor process: the non-stationary statistical multipactor theory. The non-stationary theory removes the stationarity assumption of the classical theory and, as a consequence, it is able to adequately model electron exponential growth as well as absorption processes, above and below the multipactor breakdown level. In addition, it considers both double-surface and single-surface interactions constituting a full framework for non-resonant polyphase multipactor analysis. This work formulates the new theory and validates it with numerical and experimental results with excellent agreement. 


\subsection{Multipactor theory for multi-carrier signals (See Section 4.4)}

\subsubsection{Bibliographic record}

- Authors: S. Anza and M. Mattes and C. Vicente and J. Gil and D. Raboso and V. E. Boria and B. Gimeno.

- Publication: Physics of Plasmas, American Institute of Physics, vol. 18.

- Year: 2011.

- Pages: 032105, 12 pages.

- Keywords: high-frequency discharges; plasma simulation; plasma transport processes; plasma-wall interactions; secondary electron emission.

\subsubsection{Abstract}

This work presents a new theory of multipactor under multi-carrier signals for parallel-plate geometries, assuming a homogeneous electric field and one-dimensional electron motion. It is the generalization of the non-stationary multipactor theory for single-carrier signals $\left[\mathrm{AVG}^{+} 10\right]$. It is valid for multi-carrier signals with an arbitrary number of carriers with different amplitude, arbitrary frequency and phase conditions and for any material coating. This new theory is able to model the real dynamics of the electrons during the multipactor discharge, for both single and double surface interactions. Among other parameters of the discharge, it calculates the evolution in time of the charge growth, electron absorption and creation rates as well as the instantaneous SEY and order. An extensive set of numerical tests with a PIC software has been carried out in order to validate the theory under many different conditions. This theoretical development constitutes the first multipactor theory which completely characterizes the multipactor discharge for arbitrary multi-carrier signals, setting the first step for further investigations in the field. 
3.5. Prediction of Multipactor Breakdown for Multi-carrier Applications: The Quasi-stationary Method

\subsection{Prediction of Multipactor Breakdown for Multi-carrier Applications: The Quasi-stationary Method (See Section 4.5)}

\subsubsection{Bibliographic record}

- Authors: S. Anza, C. Vicente, J. Gil, M. Mattes, D. Wolk, U. Wochner, V.E. Boria, B. Gimeno, D. Raboso.

- Publication: IEEE Transactions on Microwave Theory and Techniques, vol.60, no.7.

- Year: 2012.

- Pages: 2093-2105.

- Keywords: breakdown level; multicarrier applications; multipactor breakdown determination; multipactor electron growth models ; quasistationary method; secondary emission yield properties; single carrier signals;time varying value; microwave switches; secondary emission; vacuum breakdown.

\subsubsection{Abstract}

A new prediction algorithm for multipactor breakdown determination in multi-carrier signals is presented. This new algorithm assumes a quasi-stationary model, based on the nonstationary theory for singlecarrier signals. It determines the worst case, i.e the combination of signal phases that yields the lowest breakdown level per carrier, using multipactor electron growth models. It considers the Secondary Emission Yield properties of the material and the time-varying value of the multi-carrier signal envelope.

Several test samples have been designed and manufactured in order to assess the precision of the proposed method. The experimental results show excellent agreement with the predicted results. The quasi-stationary prediction technique yields, in general, better accuracy and more relaxed breakdown levels than the existing methods. 


\section{Chapter 4}

\section{Article list}

This chapter contains all published papers that are included in this work.

\subsection{Long-term multipactor discharge in multicarrier systems}

\subsubsection{Introduction}

Multipactor [GvE48, HW58, Vau88] is a non-linear effect that may occur in high power microwave devices at very low pressures, such as those operating in particle accelerators and satellite subsystems. A multipactor discharge is an electronic avalanche, in synchronism with the rf field, caused by secondary emission multiplication on the device walls. Its effects range from signal degradation to the complete destruction of the component.

The design of multipactor-free components is a key issue for the space telecommunications industry. Specifically, new satellite payloads operate with an extremely high number of communication channels and a still increasing power level per carrier. This leads to extremely high peak power levels in the multicarrier path of the 
spacecraft, which results in an increasing risk of multipactor discharge [SK01, GW96].

The study of multipactor in multicarrier operation is much more complex than in the single carrier case. A typical multicarrier signal is composed of several modulated carriers with small frequency separation. Their mix produces a modulated rf signal with a time varying periodic envelope. Such signals add a dynamical behavior to the multipactor process which is not well understood yet. By the time of speaking, a theory for multipactor in multicarrier operation is not well established.

Up to now, the standard adopted by the European Space Agency (ESA) for multicarrier multipactor design [EST03] is based on the "20-gap-crossing rule", which states that multipactor takes place only if the multicarrier signal envelope exceeds the breakdown voltage for a time equal or higher than the time that an electron takes to cross the gap 20 times $\left(T_{20}\right)$. This rule establishes a multipactor criterion for a single multicarrier peak. Nevertheless, the variation in time of the signal amplitude implies that, within one period of the multicarrier envelope, there are some intervals in which the amplitude of the field is above the multipactor breakdown threshold and others in which it is below. In the first case, the multipactor resonance and secondary emission yield (SEY) conditions are met, resulting in an exponential growth of the electron population. In the second case, the electrons step out of resonance and hit the walls with much lower energies and a SEY below 1, being therefore absorbed. The rate of absorption depends directly on the value of the SEY at such low energies. Therefore, if the total absorption is less than the total electron emission, there will be an overall growth or charge accumulation in one period. In such cases, since these multipactor activity and relaxation intervals, or "ON" and "OFF" intervals, are periodically repeated in time, the total electron population rises indefinitely until saturation after some periods, producing a multipactor discharge even though the 20-gap-crossing rule is accomplished.

Whereas traditional SEY models for multipactor characterization [Vau89] decrease rapidly to zero at low energies, recent works on secondary emission effect support a non-zero value of the SEY at low energies (in the range of $\mathrm{eV}$ ) due to the presence of elastic electrons $\left[\mathrm{dLPA}^{+} 06, \mathrm{FP} 02, \mathrm{CCF}^{+} 04\right.$, Sev05]. In this work, a recently 
published SEY model $\left[\mathrm{VMW}^{+} 05 \mathrm{~b}\right]$ is adopted, taking different values of the curve for low energies. On the other hand, the spread of the emission energy of the secondary emitted electrons is taken into account in both the analytical and the numerical analyses, since it results also in a spread in the electron impacting energy and, therefore, an increase in the range of valid phases at the end of the OFF interval $\left[\mathrm{SBS}^{+} 05\right]$. This presumably enhances the probability of the electron to participate in the multipactor process of the consecutive ON interval.

There are few works dealing with multipactor in multicarrier systems and accumulation. A possible justification for not existing experimental results reporting accumulation is that multipactor experiments with multicarrier signals are difficult and costly to set up due to the need of several high power amplifiers. In addition, the current multipactor detection methods do not allow to determine the nature of the multipactor discharge [GW96, $\left.\mathrm{UAI}^{+} 03, \mathrm{RLR}^{+} 94\right]$. In Ref. [SVB ${ }^{+}$03], numerical results of electron population growth for multicarrier signals and infinite parallel plates are presented, but no accumulation is reported. On the other hand, a numerical study of multipactor with a two carrier signal is given by Semenov et al. in Ref. [SK01] where accumulation is present in some cases although not treated in detail.

In this paper, a new mechanism of long-term multipactor in multicarrier applications is addressed, and the impact of the SEY properties on multipactor accumulation is investigated for the first time. Firstly, a theoretical analysis of the electron population dynamics under the action of multicarrier signal fields is developed, where the multicarrier signal envelope is simplified and approximated to be an on/off pulsed signal, which allows us to study the electron multiplication and absorption separately. The former is modelled by the classical parallel-plate two-surface single-carrier multipactor theory, whereas for the latter, and since there is no applied field, it is reasonable to assume that the electrons travel between the waveguide walls with constant velocity equal to the secondary electron emission one. This analytical model predicts the minimum average SEY needed to develop a charge accumulation process between consecutive periods of the envelope, resulting in a long-term multipactor discharge. Although the theory has been developed for pulsed signals, the an- 
alytical predictions are proven to be valid also for real multicarrier envelopes, provided that their envelope is reasonably well fitted by a pulsed signal. Finally, by means of an ad-hoc multipactor simulator for a rectangular waveguide of infinite length and multicarrier input signal, the analytical predictions are verified for different values of the SEY at low energies.

\subsubsection{Multicarrier multipactor basics}

Multipactor takes place when the electromagnetic field and the geometry of the component are such that electrons follow a resonant trajectory, impacting consecutively from wall to wall of the device, with the adequate impact energy to ensure a SEY higher than one [GvE48]. According to the classical single carrier multipactor theory [HW58, Vau88, RCD95], in order to achieve resonance, the electron travel phases between plates must be an odd number of halfperiods, i.e $n \pi$, where $n=1,3,5,7, \ldots$ is the multipactor order. The multipactor order can be analytically expressed as a function of the applied voltage and the operation frequency times the device gap, $f \times d$. Analogously, the theory sets the value of the breakdown voltage, $V_{B}$ (the minimum voltage to induce a multipactor discharge), as a function of the $f \times d$ and the SEY properties of the device material.

On the other hand, in typical communication applications, a multicarrier signal is composed by $N$ carriers, with same amplitude $V_{0}$, equally spaced in frequency $(\Delta f)$ and with relative phase $\varphi_{i}$, were $i$ stands for each individual carrier.

$$
v(t)=V_{0} \sum_{i=1}^{N} \sin \left\{2 \pi\left[f_{m}+\left(i-\frac{N+1}{2}\right) \Delta f\right] t+\varphi_{i}\right\},
$$

where $v(t)$ is the voltage of the compound signal and $f_{m}$ is the mean frequency of all carriers. Under these circumstances, the compound signal can be expressed as a single carrier with frequency $f_{m}$, modulated by an envelope, whose time evolution depends on the relative phases between carriers [Fri00]. The absolute value of the envelope is periodic with $T=1 / \Delta f$ period. Fig. 4.1 shows an example of three different envelopes of an $N=10$ carrier signal of $V_{0}=1 \mathrm{~V}$ amplitude each and with $\Delta f=40 \mathrm{MHz}$, for three phase distributions. Notice 
that the in-phase case gives the maximum envelope peak, equal to $10 \mathrm{~V}$, and the narrower main lobe. On the contrary, the triangular distribution [WSS00] gives a lower but wider main lobe, whereas the arbitrary distribution can lead to any envelope ranging from the in-phase case to the situation where the envelope amplitude is almost equally distributed throughout the whole period (very similar to single-carrier operation).

\section{Figure 4.1.}

Envelopes of a 10 carrier signal with a frequency separation of $\Delta f=40$ $\mathrm{MHz}$ and three different phase distributions with $V_{0}=1 \mathrm{~V}$. The period is $T=1 / \Delta f=25 \mathrm{~ns}$.

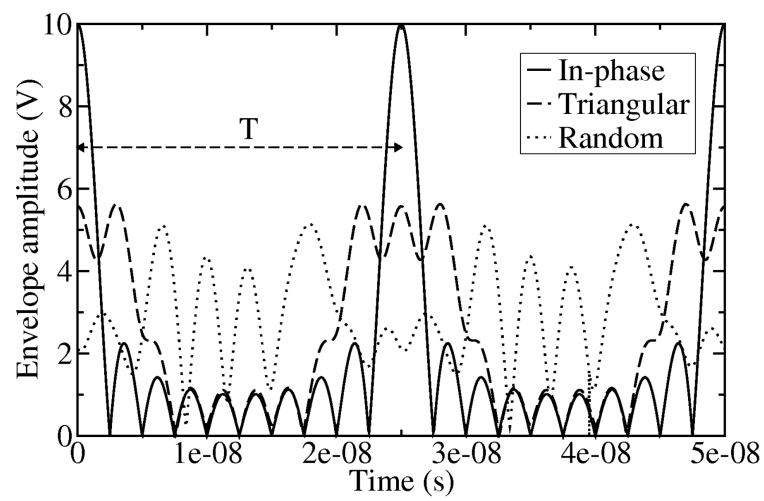

In one period of the envelope, the signal amplitude may be either higher or lower than the single-carrier multipactor breakdown threshold, $V_{B}$ (for $f_{m}$ ). Whereas in the first case there is electron multiplication, in the second one electrons are mainly absorbed. These two processes are repeated periodically with period $T$, where the rate of electron creation and absorption is governed mainly by the geometry of the device, the material SEY properties and the amplitude of the envelope.

It is not straight-forward to define a multipactor criterion under such conditions. In the classical single-carrier case, if the amplitude of the field is higher than $V_{B}$, the electron population grows indefinitely until saturation causing certainly a multipactor discharge. However, in the multicarrier case, the electron population shows peaks and valleys, not being clear the correspondence between the peak height and the existence of the discharge.

The 20-gap-crossing rule [EST03] establishes a multipactor criterion for multicarrier signals based in the peak height and duration of the multicarrier signal envelope. This rule has been obtained from 
numerical simulations and claims that multipactor takes place if the ON interval is longer than the 20 -gap-crossing time, $T_{20}$, which can be expressed as

$$
T_{20}=\frac{10 n}{f_{m}},
$$

being $n$ the multipactor order and $f_{m}$ the mean frequency of the multicarrier signal. Physically, $T_{20}$ corresponds to the time that an electron, under resonance conditions, takes to cross the gap 20 times. This criterion only considers the electron growth in one single event or envelope period, disregarding possible inter-period charge accumulation. In such cases the electron population curve may show an increasing exponential trend, thus producing a long-term multipactor discharge.

\subsubsection{SEY model}

One of the most employed SEY models is the Vaughan's model [Vau89]. Vaughan proposed a parametric formula of the SEY which allows to fit experimental data.

$$
\begin{array}{ll}
\sigma(W, \theta)=\sigma_{\max }(\theta)\left(u e^{1-u}\right)^{k} & \text { for } \quad u \leq 3.6 \\
\sigma(W, \theta)=\sigma_{\max }(\theta) 1.125 / u^{0.35} & \text { for } \quad u>3.6
\end{array},
$$

where

$$
\begin{aligned}
& u=\frac{W-W_{0}}{W_{\max }(\theta)-W_{0}}, \\
& k=0.56 \text { for } u<1, \\
& k=0.25 \text { for } 1<u<3.6, \\
& \sigma_{\max }(\theta)=\sigma_{\max }\left(1+k_{E} \theta^{2} / 2 \pi\right), \\
& W_{\max }(\theta)=W_{\max }\left(1+k_{\theta} \theta^{2} / 2 \pi\right) .
\end{aligned}
$$

These equations give the value of the SEY $\sigma(W, \theta)$ for an electron impacting energy $W$ and incident angle $\theta$ with respect to the normal, where $W_{0}=12.5 \mathrm{eV}, k_{E}$ and $k_{\theta}$ are surface dependent parameters, and $W_{\max }$ is the energy at which the maximum SEY $\sigma_{\max }$ is attained.

Vaughan's formula fits reasonably well the experimental data and has been widely used for modelling multipactor. However, it does not consider the existence of low energy elastic electrons. The presence of this kind of electrons may reduce the rate of absorption of the electron population during the OFF intervals of the multicarrier envelope, thus facilitating inter-period accumulation. 
On the other hand, the Furman and Pivi SEY statistical model [FP02] takes into account the different nature of the electrons contributing to SEY, resulting in a SEY tending to 0.5 at low energies instead of 0 , which means that half of the low energy electrons are reflected and the other half are absorbed. Recent SEY measurements undertaken by Cimino et al. $\left[\mathrm{CCF}^{+} 04\right]$ suggest that Furman and Pivi model underestimates the importance of low energy electrons, due mainly to the lack of resolution in the experimental measurements of SEY at low energies. Cimino et al. conclude that the SEY for most materials tends to one in the limit of zero impact energy, i.e. most of the low energy electrons are reflected.

In this work, the SEY model is a modification of the Vaughan's model $\left[\mathrm{VMW}^{+}\right.$05b] shown in Fig. 4.2. According to the literature, the values of the curve at low energies $\left(\sigma_{0}\right)$ have been taken as $0,0.5$ and 1 , in order to assess its influence on the multipactor breakdown levels.

\section{Figure 4.2.}

SEY model for silver used in this work, extracted from Ref. $\left[\mathrm{VMW}^{+} 05 \mathrm{~b}\right]$, with non-zero SEY at low energies $\left(\sigma_{0}\right)$. In this case $\sigma_{0}=0.5$.

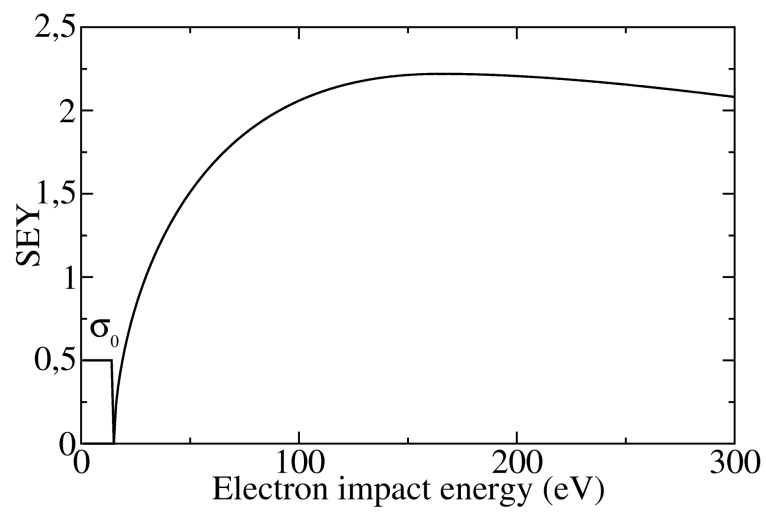

With regard to the secondary electron release velocity and angle (with respect to the normal of the surface), $v_{S E}$ and $\theta_{S E}$, they are assumed to be random variables with maxwellian and cosine law distributions, respectively $\left[\mathrm{VMW}^{+} 05 \mathrm{~b}, \mathrm{VSS} 04, \mathrm{Gre02}\right]$. For instance, the distribution of $v_{S E}$ is

$$
f\left(v_{S E}\right) \propto v_{S E} \exp \left(-\frac{\left(v_{S E}-v_{0}\right)^{2}}{2 v_{T}^{2}}\right),
$$


where $v_{0}$ and $v_{T}$ are the emission velocity mean and the thermal spread, respectively. On the other hand, the distribution of $\theta_{S E}$ is given by

$$
f\left(\theta_{S E}\right)=\sin ^{2}\left(\theta_{S E}\right) .
$$

The emission angle with respect the surface tangential, $\phi_{S E}$, is uniformly distributed from 0 to $2 \pi$.

\subsubsection{Accumulation model}

In order to investigate the charge accumulation process in the longterm multipactor discharge, it becomes necessary to study separately the electron multiplication and absorption processes during the ON and OFF times, respectively. Since the shape of the multicarrier signal envelope depends on many factors, such as the number of carriers, frequency separation and carrier phasing, it is an extremely difficult task to study the time evolution of the electron population for the general case. Therefore, let us consider a simplified envelope shape, the rectangular pulsed signal $v(t)=A(t) \sin (2 \pi f t)$, with

$$
A(t)=\left\{\begin{array}{cc}
V_{0}, & l\left(T_{o n}+T_{o f f}\right)<t<(l+1) T_{o n}+l T_{o f f} \\
0, & (l+1) T_{o n}+l T_{o f f}<t<(l+1)\left(T_{o n}+T_{o f f}\right)
\end{array},\right.
$$

where $l$ is the envelope period number, and $V_{0}$ and $f$ are the signal amplitude and frequency, respectively. Under the pulsed signal approximation, the multicarrier-signal presents a uniform ON interval during a time $T_{o n}=\frac{C_{o n}}{2 f}$ and an OFF interval, with total absence of field, during a time $T_{o f f}=\frac{C_{o f f}}{2 f}$, where $C_{o n}$ and $C_{o f f}$ are the number of cycles for the ON and OFF intervals, respectively. An example of such an envelope for a particular combination of $C_{o n}$ and $C_{o f f}$ is shown in Fig. 4.3.

The electrons are assumed to be in resonance during the ON interval and, therefore, it is expected that they impact against the surfaces with approximately the same energy and thus nearly the same SEY, $\sigma_{o n}$. According to the classical multipactor theory, the number of electrons in the $k$-th cycle, $N(k)$, during the ON interval is

$$
N(k)=N_{i} \Gamma(k) \text { for } 0 \leq k \leq C_{o n},
$$




\section{Figure 4.3.}

Pulsed signal envelope with $C_{o n}=276$ cycles and $C_{o f f}=224$ cycles.

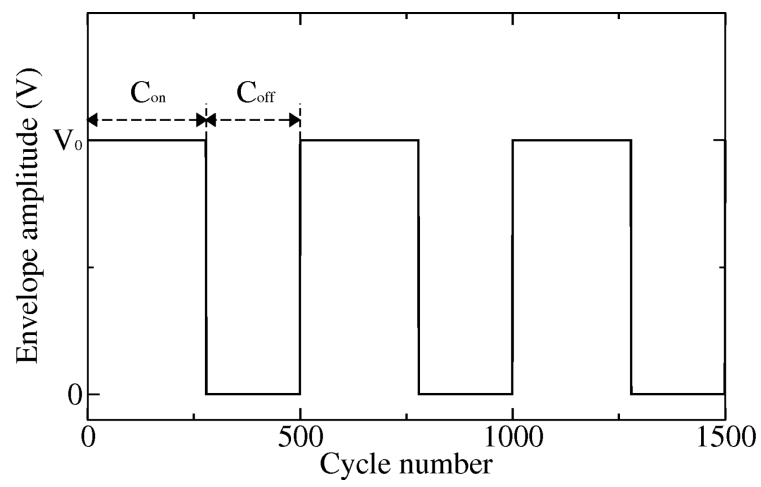

where $N_{i}$ is the initial number of electrons (for $k=0$ ) and $\Gamma(k)$ is the electron multiplication factor, defined for the ON interval as

$$
\Gamma(k)=\sigma_{o n}{ }^{\frac{k}{n}} \text { for } \quad 0 \leq k \leq C_{o n},
$$

where $k / n$ is the number of impacts for a single electron in $k$ cycles, being $n$ the two-surface multipactor order. Therefore, at the end of the ON interval the multiplication factor is

$$
\Gamma_{o n}=\Gamma\left(C_{o n}\right)=\sigma_{o n} \frac{C_{o n}}{n} .
$$

This expression does not consider the effects of the space charge, which is the main responsible for the saturation of the multipactor discharge [Vau88]. However, its effects manifest for high electron densities [Riy97] and, since a typical multipactor discharge initiates with a low number of free electrons, space charge can be neglected at the first stages, remaining Eq. 4.9 valid for the purpose of this work.

During the OFF interval, there is no applied field and, therefore, after the first impact, the surviving electrons travel from side to side of the device with a constant velocity equal to the secondary emission velocity. The component of such velocity perpendicular to the surface is given by $v_{\perp}=\cos \left(\theta_{S E}\right) v_{S E}$. Hence, $v_{\perp}$ is a random variable defined by the statistics of the electron emission velocity, $v_{S E}$, and angle, $\theta_{S E}$. Since the electrons are not accelerated, the impact energy is equal to the emission energy, which, for typical $v_{S E}$ values (energies in the range of few eV), leads to a $\mathrm{SEY}$ equal to $\sigma_{0}$ (see Fig. 4.2). 
Under such circumstances, the electron population in the cycle $k+1$, $N(k+1)$, is given by

$$
N(k+1)=N(k)+i(k)\left(\sigma_{0}-1\right) .
$$

Here $i(k)$ is the total number of impacts during the cycle $k$. From Eq. (4.10), the effective SEY per cycle, $\sigma_{e f f}$ is defined as

$$
\sigma_{e f f} \equiv\langle N(k+1) / N(k)\rangle=1+\left\langle\frac{i(k)}{N(k)}\right\rangle\left(\sigma_{0}-1\right),
$$

being $\langle\cdot\rangle$ the statistical expectation operator and $\left\langle\frac{i(k)}{N(k)}\right\rangle$ the average number of impacts per electron and cycle.

On the other hand, an electron leaving one of the surfaces with constant velocity $v_{\perp}$ crosses the gap, $d$ in a time $d / v_{\perp}$. Therefore, the average number of cycles between two consecutive impacts, $C$, can be expressed as follows.

$$
C=\left\langle\frac{1}{v_{\perp}}\right\rangle 2 f d .
$$

Notice that, in general, $\left\langle\frac{1}{v_{\perp}}\right\rangle \neq \frac{1}{\left\langle v_{\perp}\right\rangle}$.

Then, taking into account that $\left\langle\frac{i(k)}{N(k)}\right\rangle=\frac{1}{C}$,

$$
\left\langle\frac{i(k)}{N(k)}\right\rangle=\frac{1}{2 f d\left\langle\frac{1}{v_{\perp}}\right\rangle} .
$$

Analogously to the ON interval, the number of electrons during the OFF interval is given by

$$
N(k)=N_{f} \Gamma(k) \text { for } C_{o n}<k \leq C_{o n}+C_{o f f},
$$

where $N_{f}$ is the number of electrons at the end of the ON interval $\left[N\left(C_{o n}\right)\right]$. Substituting Eq. (4.13) in Eq. (4.11), the electron multiplication factor during the OFF interval, can be written as

$$
\begin{gathered}
\Gamma(k)=\sigma_{e f f}^{k-C_{o n}}=\left[1+\frac{\sigma_{0}-1}{2 f d\left\langle\frac{1}{v_{\perp}}\right\rangle}\right]^{k-C_{o n}} . \\
\quad \text { for } C_{o n}<k \leq C_{o n}+C_{o f f}
\end{gathered}
$$


Therefore, at the end of the OFF interval the multiplication factor is

$$
\Gamma_{o f f}=\Gamma\left(C_{o n}+C_{o f f}\right)=\left[1+\frac{\sigma_{0}-1}{2 f d\left\langle\frac{1}{v_{\perp}}\right\rangle}\right]^{C_{o f f}},
$$

which manifests the role of the SEY at low energies on the absorption rate. A $\sigma_{0}=0$ implies the maximum absorption rate (minimum $\left.\Gamma_{o f f}\right)$, whereas for $\sigma_{0}=1$, all impacting electrons are reflected and there is no absorption at all $\left(\Gamma_{o f f}=1\right)$, the electron population remaining constant.

At this point (end of the OFF interval), the surviving electrons are distributed along the waveguide with velocities following the secondary emission velocity distribution. Only the fraction of those which have the correct combination of position and velocity, in order to impact with favorable phase, will participate in the consecutive ON interval. This fraction $\alpha(n)$ varies with the multipactor mode $n$.

Fig. 4.4 shows the values of $\alpha(n)$ for different multipactor orders and $\sigma_{0}$. The curve has been computed performing several runs of electron population versus time, calculated numerically with the multipactor simulator used in Sec. 4.1.5. There are no relevant differences for the three values of $\sigma_{0}$, although higher values of it tend to rise the curve towards a higher $\alpha(n)$. The curve tends to one for increasing mode due to the fact that higher $f \times d$ products allow the simultaneous existence of different multipactor modes and hybrid modes $\left[\mathrm{KBS}^{+} 02\right.$, Gil92], which, together with the spread of the secondary emission velocity and the subsequent broadening of the range of valid phases $\left[\mathrm{SBS}^{+} 05\right]$, enhances the probability for each electron to participate in the discharge. Indeed, for high values of $n$ (above $n=13) \alpha$ can be safely assumed to be one.

Finally, in order to accumulate charge, the total electron growth must hold $\Gamma_{o n} \Gamma_{o f f} \alpha(n) \geq 1$, which leads, along with Eqs. (4.9) and (4.16), to the minimum average SEY value during the ON interval producing accumulation, $\sigma_{o n, \min }\left(\right.$ when $\left.\Gamma_{o n} \Gamma_{o f f} \alpha(n)=1\right)$, given by

$$
\sigma_{o n, \min }=\left(\frac{1}{\alpha(n)}\right)^{n / C_{o n}}\left(1+\frac{\sigma_{0}-1}{2 f d\left\langle\frac{1}{v_{\perp}}\right\rangle}\right)^{-n C_{o f f} / C_{o n}} .
$$




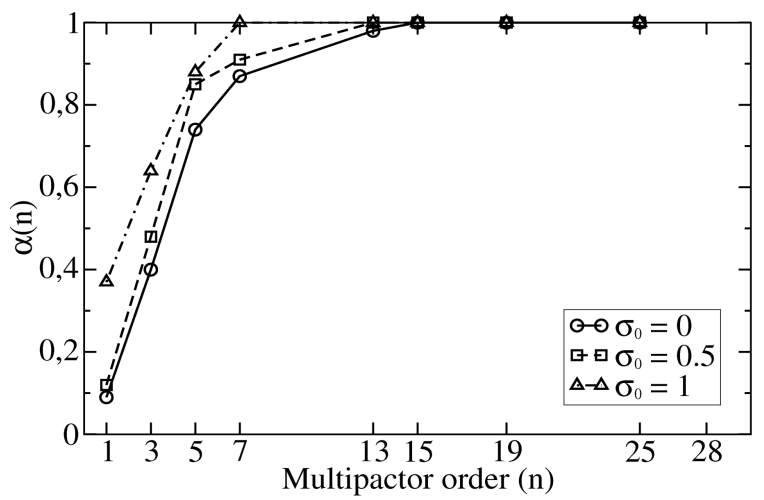

Figure 4.4.

Fraction $\alpha(n)$ of electrons participating in the multipactor process at the beginning of the ON interval. The curve has been numerically calculated for three different values of $\sigma_{0}$.

The correction factor, $(1 / \alpha(n))^{n / C_{o n}}$, of Eq. 4.17 , is plotted in Fig. 4.5 for $\sigma_{0}=0$. Note that in most applications typically $n / C_{o n} \approx$ $1 / 20$ (following the 20-gap-crossing rule), which leads to values of this correction factor very close to one. Hence, it is interesting to see that, according to this model and neglecting the contribution of $\alpha(n)$, the long-term multipactor threshold, $\sigma_{o n, \min }$, depends only on $\sigma_{0}, f \times d, n$, and $C_{o f f} / C_{o n}=T_{o f f} / T_{o n}$, being always $\sigma_{o n, \min } \geq 1$. The limit cases of $\sigma_{0}=0$ and $\sigma_{0}=1$, yield the maximum value of $\sigma_{\text {on,min }}$ and the minimum (equal to unity), respectively. This implies that the higher the value of $\sigma_{0}$, the lower the long-term multipactor threshold, which is obvious since it also implies a lower absorption rate during the $\mathrm{OFF}$ interval. Similarly, the $C_{\text {off }} / C_{o n}$ ratio also affects the threshold in a very intuitive manner. The higher the ratio, the higher the $\sigma_{o n, m i n}$, since the absorption process duration increases with respect to the multiplication one.

Therefore, Eq. 4.17 sets the long-term multipactor threshold. If a certain field envelope ensures $\sigma_{o n}>\sigma_{o n, m i n}$, there will be charge accumulation and the subsequent multipactor discharge. However, the SEY during the ON interval, $\sigma_{o n}$, depends on the envelope amplitude during the ON time, since it sets the electron impact energy, and thus the SEY. This relationship cannot be easily established but for voltages close to the multipactor susceptibility threshold it is expected that a higher ON voltage also implies a higher $\sigma_{o n}$.

As stated before, the modelling of the general case for a multicarrier signal envelope is a very challenging task. However, provided 
Figure 4.5.

Correction against multipactor order for fixed values of $n / C_{o n}$ and $\sigma_{0}=0$.

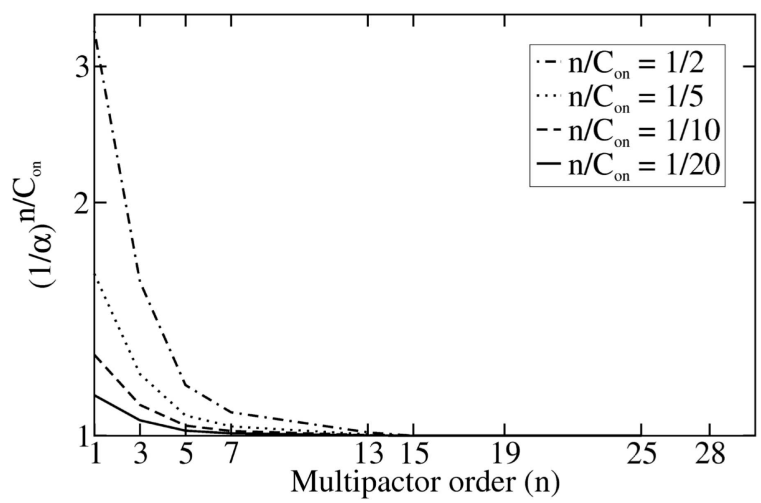

that the envelope has differentiated $\mathrm{ON}$ and $\mathrm{OFF}$ intervals within a period with a reasonably uniform voltage for the ON time and a very low voltage for the OFF time, this pulsed signal accumulation model can be applied. For instance, the first two phase distributions of Fig. 4.1 meet the conditions to be modelled by a pulsed signal, whereas the arbitrary phasing of the third signal spreads its envelope throughout the whole period and thus, no differentiation between $\mathrm{ON}$ and OFF intervals can be done.

\subsubsection{Numerical results}

Several numerical simulations have been carried out using the analytical solution of the electromagnetic 3D fields in a rectangular waveguide of infinite length for the fundamental mode $\mathrm{TE}_{10}$. An X-band waveguide with width $a=22.86 \mathrm{~mm}$ and height $b=2 \mathrm{~mm}$ has been chosen for the testing. The working frequency $\left(f_{m}\right.$ for multicarrier operation) is $10 \mathrm{GHz}$, being then $f \times d=20 \mathrm{GHz} \cdot \mathrm{mm}, n=15$ and $T_{20}=15 \mathrm{~ns}$. The 3D trajectories have been determined for each individual electron with the Velocity-Verlet algorithm, using the method of Ref. [VMW $\left.{ }^{+} 05 b\right]$.

The chosen material is silver with the SEY parameters extracted from Ref. [EST03] where the SEY model of Ref. [VMW ${ }^{+}$05b] has been applied. For a maxwellian secondary emission energy with a thermal spread of $3 \mathrm{eV}$, and a cosine law distribution for the emission angle, the mean value $\left\langle\frac{1}{v_{\perp}}\right\rangle$ of Eq. (4.12) results in $2.6 \times 10^{-6} \mathrm{sm}^{-1}$. 


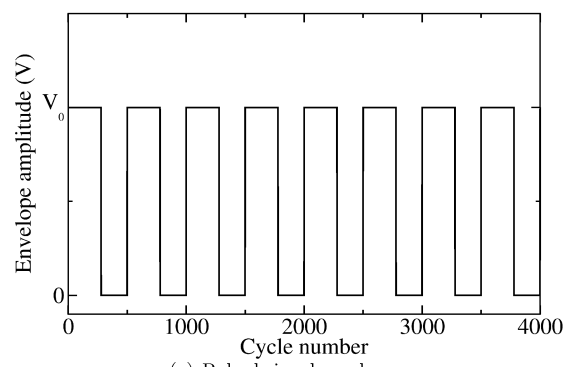

(a) Pulsed signal envelope.

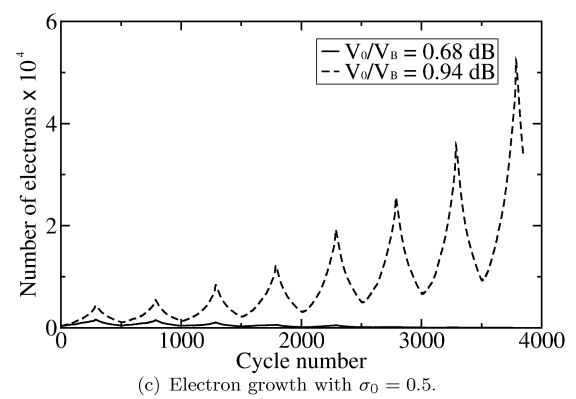

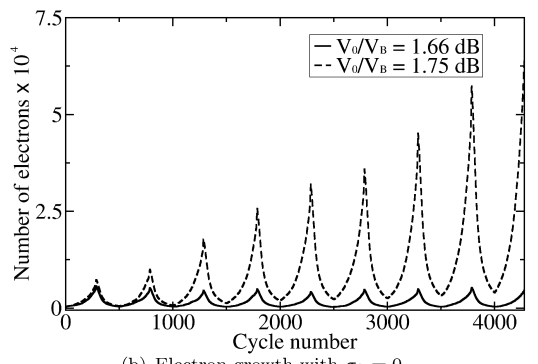

(b) Electron growth with $\sigma_{0}=0$.

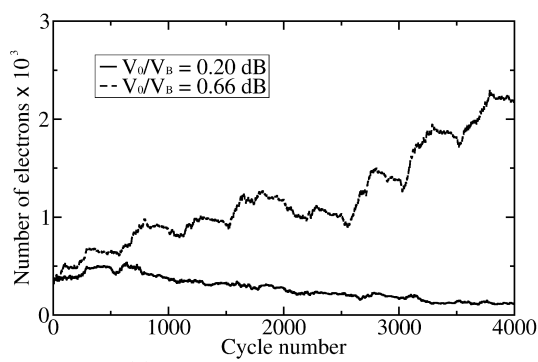

(d) Electron growth with $\sigma_{0}=1$.

Figure 4.6. (a) Pulsed signal envelope, with a period $T=25 \mathrm{~ns}$, $T_{o n}=13.8 \mathrm{~ns}$ and $T_{o f f}=11.2 \mathrm{~ns}$. $V_{0}$ is the ON voltage. (b) to (d) Electron population growth for different $\sigma_{0}$. The curves have been plotted for different values of $V_{0}$ to breakdown voltage ratio, $V_{0} / V_{B}$, expressed in $\mathrm{dB}$.

The numerical tests have been run with the three possible values of $\sigma_{0}$ proposed by the existing literature and mentioned in Sec. 4.1.3, i.e. $0,0.5$ and 1. Two kind of input signals have been used, rectangular pulsed and multicarrier, with the parameters shown in Fig. 4.6 and Fig. 4.8, respectively.

The main reason to choose such signals is twofold. First, both signals have equivalent $\mathrm{ON}$ and $\mathrm{OFF}$ times in order to be compared. Second, the ON time should always be less than $T_{20}$ in order to ensure that the 20-gap-crossing rule is always accomplished. According to such a rule, such signals should never develop a multipactor discharge in a single peak. 


\subsubsection{Single carrier signal}

The multipactor breakdown voltage has been numerically computed for the single carrier signal. Table 4.1 shows the influence of $\sigma_{0}$ on the breakdown voltage along with the fitted value from the experimental results of Ref. [EST03].

Table 4.1.

Breakdown voltage for single carrier.

\begin{tabular}{ccccc}
$\sigma_{0}$ & 0 & 0.5 & 1 & Experimental $^{*}$ \\
\hline$V_{B}(\mathrm{~V})$ & 1635 & 1480 & 1173 & 1400 \\
\hline \multicolumn{5}{c}{ Extracted from Ref. [EST03]. }
\end{tabular}

As it can be observed, the numerical results for $\sigma_{0}=0.5$ offer the closer value to the experimental one, which agree with the results given in Ref. $\left[\mathrm{VMW}^{+}\right.$05a]. Notice that using a zero low-energy SEY implies an overestimation of the multipactor threshold.

\subsubsection{Pulsed signal}

The simulator has been run for each $\sigma_{0}$ and for a pulsed signal with $C_{o n}=276, C_{o f f}=224$, and increasing input voltage $V_{0}$, shown in Fig. 4.6(a). As expected, for low values of $V_{0}$ (implies low $\sigma_{o n}$ ) no accumulation is observed, but beyond a certain voltage, here defined as the accumulation voltage threshold $V_{a c c}$, the time evolution of the electron population shows a noticeable inter-period accumulation. This can be seen in Fig. 4.6(b), Fig. 4.6(c) and Fig. 4.6(d), which show the electron population growth with time for two different levels of $V_{0}$, below and equal to $V_{a c c}$. Notice that the creation or absorption of electrons coincide with the ON and OFF periods of the envelope, respectively. Accumulation has been observed for the three values of $\sigma_{0}$.

Table 4.2. Analytical and numerical results for pulsed signal.

\begin{tabular}{ccccc}
$\sigma_{0}$ & $\begin{array}{c}\sigma_{\text {on,min }} \\
\text { Eq. }(4.17))\end{array}$ & $\begin{array}{c}\sigma_{\text {on,min }} \\
(\text { num. })\end{array}$ & $V_{\text {acc }}(\mathrm{V})$ & $\frac{V_{\text {acc }}}{V_{B}}(\mathrm{~dB})$ \\
\hline 0 & 1.12 & 1.14 & 2000 & 1.75 \\
0.5 & 1.06 & 1.09 & 1650 & 0.94 \\
1 & 1.00 & 1.02 & 1265 & 0.66 \\
\hline
\end{tabular}




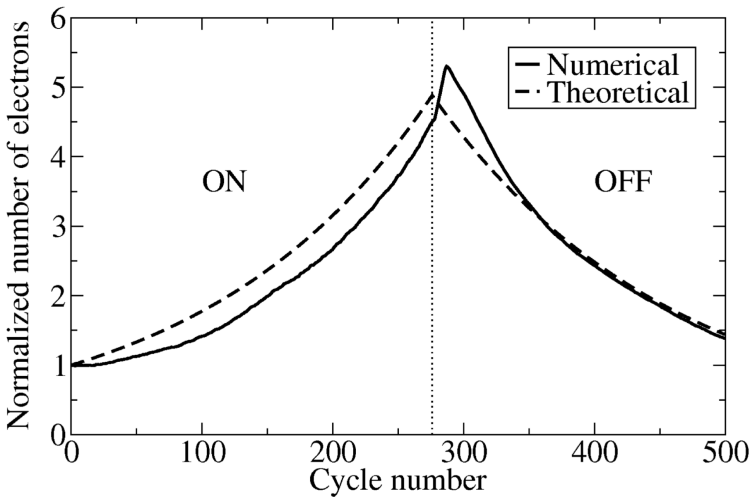

Figure 4.7.

Electron growth for a single period of the pulsed signal for $\sigma_{0}=0.5$. The $\mathrm{ON}$ and $\mathrm{OFF}$ intervals are delimited by the vertical dotted line.

The average value of $\sigma_{o n, \min }$ for all impacting electrons during the $\mathrm{ON}$ interval has been numerically computed for $V_{0}=V_{a c c}$ and compared with the minimum value predicted by Eq. (4.17), assuming $\alpha(n)=1$ since the mode order of the discharge is considerably high $(n=15)$. Table 4.2 shows these results for all values of $\sigma_{0}$. A good agreement between numerical results and predictions is found. Moreover, the accumulation to breakdown margin $V_{a c c} / V_{B}$ reduces as $\sigma_{0}$ increases, which implies that higher low-energy SEY values increases the susceptibility of the system to develop a multipactor accumulation process.

Fig. 4.7 shows the electron growth during a single period of the pulsed signal envelope assuming a unique initial electron and $\sigma_{0}=$ 0.5. Both theoretical and numerical curves are plotted. The theoretical curve is obtained using Eqs. 4.7, 4.8, 4.14 and 4.15, with the value for $\sigma_{o n}$ given in Table 4.2 and $\alpha(n)=1$. The numerical simulation shows that the electrons keep on multiplicating few cycles after the end of the ON interval and then the absorption process starts. This is because at the end of the ON interval there is a high number of energetic electrons that are able to generate secondaries during the following cycles even if no field is present, thus maintaining the growth rate. On the other hand, the electron growth at the beginning of the ON interval in the numerical simulation is slightly delayed with respect to the theory. This is caused by the initial nonresonant electrons which prevent the population to grow at full pace until complete resonance is achieved. This delay or dwell-time has 


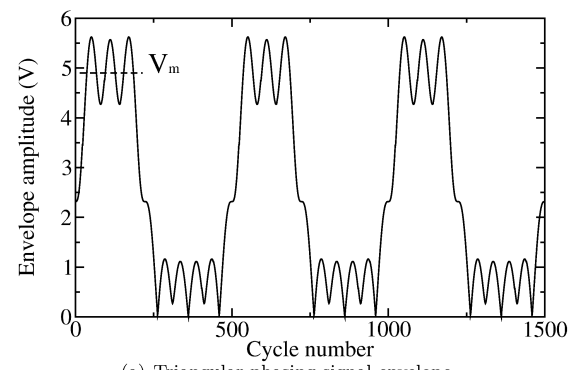

(a) Triangular phasing signal envelope.

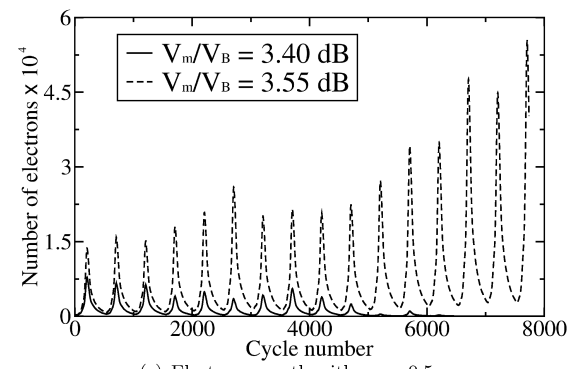

(c) Electron growth with $\sigma_{0}=0.5$.

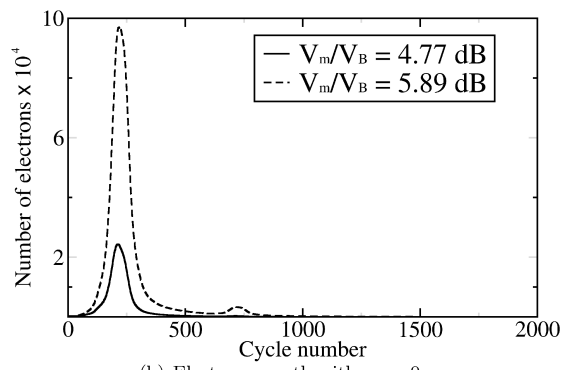

(b) Electron growth with $\sigma_{0}=0$.

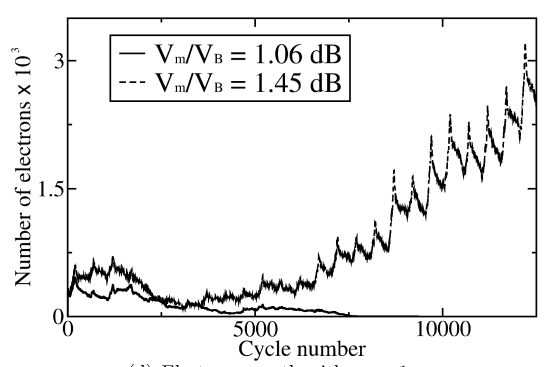

(d) Electron growth with $\sigma_{0}=1$.

Figure 4.8. (a) Multicarrier signal envelope $\left(V_{0}=1 \mathrm{~V}\right.$ in this example). It consists of $N=10$ carriers, with triangular phasing [WSS00] and $\Delta f=40 \mathrm{MHz}$, which ensures an envelope with a period of $25 \mathrm{~ns}$ and a main lobe width (from zeros) equal to $13.8 \mathrm{~ns}$. $V_{m}$ is the mean voltage of the envelope main lobe $\left(V_{m}=4.9 \mathrm{~V}\right.$ for $\left.V_{0}=1 \mathrm{~V}\right)$, plotted in dotted line. (b) to (d) Electron population growth for different $\sigma_{0}$. The curves have been plotted for different values of $V_{m}$ to breakdown voltage ratio, $V_{m} / V_{B}$, expressed in $\mathrm{dB}$. 
been also reported in experimental multipactor tests [RW95]. Nevertheless, although introducing some deviation, these effects do not change significantly the overall agreement between theory and simulations, being the electron creation and absorption rates during the $\mathrm{ON}$ and OFF intervals well fitted by the theory.

\subsubsection{Multicarrier signal}

Similarly to the pulsed signal tests, the simulator has been run for the multicarrier case and for each $\sigma_{0}$, with an input signal composed of $N=10$ carrier with triangular phasing [WSS00], $\Delta f=40 \mathrm{MHz}$ and increasing envelope mean lobe voltage, $V_{m}$ [see Fig. 4.8(a)]. Fig. 4.8(b) to Fig. 4.8(d) show the electron population growth with time. No accumulation has been observed for $\sigma_{0}=0$ and $V_{m}$ values up to 8 dB higher than $V_{B}$ which are far above from the nominal $n=15$ multipactor order limits.

Table 4.3 shows the predictions and numerical results for the minimum SEY. The predictions were computed using Eq. (4.17) with $\alpha(n)=1$ and assuming an equivalent pulsed signal to model the multicarrier envelope, with the reasonable values of $T_{o n}=\frac{1}{3} T$ and $T_{\text {off }}=\frac{2}{3} T$, being $T=25 \mathrm{~ns}$ the envelope period.

Table 4.3. Analytical and numerical results for multicarrier signal.

\begin{tabular}{ccccc}
$\sigma_{0}$ & $\begin{array}{c}\sigma_{\text {on,min }} \\
\text { Eq. }(4.17))\end{array}$ & $\begin{array}{c}\sigma_{\text {on,min }} \\
(\text { num. })\end{array}$ & $V_{\text {acc }}(\mathrm{V})$ & $\frac{V_{a c c}}{V_{B}}(\mathrm{~dB})$ \\
\hline 0 & 1.34 & - & - & - \\
0.5 & 1.16 & 1.24 & 2227 & 3.55 \\
1 & 1.00 & 1.09 & 1386 & 1.45 \\
\hline
\end{tabular}

Since the field is not zero during the OFF interval and the electrons are accelerated out of resonance, the absorption during the OFF interval increases. This produces a slightly higher difference between theory and numerical results compared to the pulsed signal counterpart. However, a good agreement among predicted and numerical results is still observed. The fact that there is no accumulation for $\sigma_{0}=0$ leads to the conclusion that, with independence on the value of the input voltage (provided that the multipactor mode order does not 
change), the mean SEY is not higher than the minimum established by the prediction, $\sigma_{o n, \min }=1.34$.

It is important to remark that according to the 20-gap-crossing rule, both the pulsed and multicarrier signals should not develop a multipactor discharge in any case. However, the simulations show that beyond $V_{a c c}$, the electron population increases indefinitely towards saturation due to inter-period accumulation, developing a longterm multipactor discharge.

\subsubsection{Conclusions}

By taking some simplifications on the multicarrier envelope, an analytical model of the electron population dynamics under slow varying amplitude fields has been developed. As a result, the minimum SEY during the ON period necessary to develop accumulation has been derived as a function of the multicarrier signal, material properties and multipactor order parameters.

The analytical model has been verified with a multipactor numerical simulator for a rectangular waveguide. Although the analytical model assumes an on/off pulsed signal envelope shape, the numerical results demonstrate that it is also valid for more general multicarrier envelopes provided that they have a relatively uniform amplitude during the ON time, and a very low amplitude during the OFF time.

It has been demonstrated that, even if the 20-gap-crossing rule is accomplished, accumulation effect between periods of the multicarrier signal envelope may raise the electron population to significant levels producing long-term multipactor. Furthermore, a zero value of the SEY at low energies $\sigma_{0}=0$ overestimates the accumulation to breakdown margin, $V_{a c c} / V_{B}$, since it increases as $\sigma_{0}$ approaches to zero, reaching even infinity for the multicarrier signal used.

To the knowledge of the authors, this work provides the first theoretical study for long-term multipactor in multicarrier systems, which allows to establish a SEY threshold and predict charge accumulation for a wide variety of situations. 
4 
4.2. RF breakdown prediction for microwave passive components in multi-carrier operation

\subsection{RF breakdown prediction for microwave passive components in multi-carrier operation}

\subsubsection{Introduction}

Nowadays, the continuously increasing number of channels in multiplexers for satellite applications forces space industry to face the problem of power handling during the design process. This leads to extremely high power levels within the devices and increases the risk of RF breakdown due to corona discharge or multipactor breakdown.

The multipactor effect is an electron discharge which takes place between dielectric or metal surfaces in the presence of a high-level and high-frequency electromagnetic field and in vacuum conditions [Vau88]. Multipactor is a matter of special importance in microwave circuits for space communications and particle accelerators.

A lot is known about multipactor breakdown for the single carrier case and parallel plate configurations (see, for example, [WJ89]). However, the prediction of multipactor in multi-carrier operation is much more complex, due to the time variability of the RF amplitude [EST03, $\left.\mathrm{AVG}^{+} 07\right]$.

This work presents a novel software for multipactor analysis in multi-carrier operation, inside rectangular waveguide-based devices, e.g. filters, combining integral equations and the method of moments (MoM), a particle in cell code (PIC) and a finite-difference timedomain (FDTD) scheme. Section 4.2.2 introduces some multipactor basics. Sections 4.2.3 and 4.2.4 present the electromagnetic field computation and electron trajectories and space charge modeling. Finally, some results and conclusions are presented in Sections 4.2.5 and 4.2.6, respectively.

\subsubsection{Multipactor Basics}

Multipactor takes place when the electromagnetic field and the geometry of the component are such that electrons follow a resonant trajectory, impacting consecutively from wall to wall of the device, with the adequate impact energy to ensure a secondary emission yield (SEY) higher than 1. 
According to the classical single carrier multipactor theory [Vau88], in order to achieve resonance, the electron travel phases between plates must be an odd number of half-periods, i.e. $n \pi$, where $n=1,3,5,7, \ldots$ is the multipactor order. The theory sets the value of the breakdown voltage, $V_{B}$ (the minimum voltage to induce a multipactor discharge), as a function of the frequency times the device gap $f \times d$ and the SEY properties of the device material.

On the other hand, in typical communication applications, a multi-carrier signal is composed of $N$ carriers having the same amplitude $V_{0}$, equally spaced in frequency $(\Delta f)$ and with relative phases $\phi_{i}$, where $i$ stands for each individual carrier

$$
V(t)=V 0 \sum_{i=1}^{N} \sin \left\{2 \pi\left[f_{n}+\left(i-\left(\frac{N+1}{2}\right)\right) \Delta f\right] t+\phi_{i}\right\},
$$

$V(t)$ being the voltage of the compound signal and $f_{m}$ the mean frequency of all carriers. Under these circumstances, the compound signal can be treated as a single carrier signal with frequency $f_{m}$, modulated by an envelope, whose time evolution depends on the relative phases between the carriers. The absolute value of the envelope is periodic with a period of $T=1 / \Delta f$.

The single carrier case is easy to characterize because when multipactor conditions are accomplished the electron avalanche will reach its saturation level since the single carrier signal has constant amplitude $\left[\mathrm{VMW}^{+} 05 \mathrm{~b}\right]$.

On the contrary, the amplitude of a multi-carrier signal varies with time periodically and the multipactor discharge can be understood as periodic electron avalanches, which takes place whenever the signal envelope is higher than $V_{B}$ ("ON" intervals), with intercalated periods of inactivity when the amplitude is too low to hold a discharge ("OFF" intervals) $\left[\mathrm{AVG}^{+} 07\right]$.

The European Space Agency (ESA) employs the 20-gap-crossing rule as the standard for multipactor design and test [EST03], which establishes that multipactor takes place if the ON interval is longer than the 20-gap-crossing time, $T_{20}=10 n / f_{m}$.

However, the 20-gap-crossing rule only covers single-period discharges and does not offer a good understanding of the real processes that lay underneath. In this scenario, multipactor numerical sim- 
4.2. RF breakdown prediction for microwave passive components in multi-carrier operation

ulations taking into account the electronic space charge give very valuable information and ultimately may lead to the redefinition of the criterion.

\subsubsection{Field Analysis of Microwave Devices}

Microwave devices such as filters and multiplexers are typically operated in a narrow frequency range. For this reason, the electromagnetic field distribution needed to simulate the RF breakdown is most efficiently computed in the frequency domain. Assuming a timeharmonic excitation, spatial and time dependency can be separated:

$$
\vec{E}(\vec{r}, \omega, t)=\underline{\vec{E}}(\vec{r}, \omega) \mathrm{e}^{j \omega t} \quad \text { and } \quad \vec{H}(\vec{r}, \omega, t)=\underline{\vec{H}}(\vec{r}, \omega) \mathrm{e}^{j \omega t}
$$

The phasors $\underline{\vec{E}}(\vec{r}, \omega)$ and $\underline{\vec{H}}(\vec{r}, \omega)$ are computed inside the device under test for the frequency $\omega$ of interest.

To analyse a certain structure a divide-and-conquer approach is applied [Mat03]. For this, the device is separated into pieces of canonical shape, e.g. rectangular waveguides, as in Fig. 4.9. Then the response of each transition between two different waveguides is computed and characterized by means of an impedance matrix. The individual impedance matrices are then connected using microwave network theory [Oli84] to obtain the overall response of the device.

In order to obtain an impedance matrix representation, an integral equation is settled. This is done by imposing the continuity of the magnetic field in the aperture (see, for instance, the first discontinuity in the upper right picture in Fig. 4.9)

$$
\vec{n} \times\left(\underline{\vec{H}}^{(1)}-\underline{\vec{H}}^{(2)}\right)=0 .
$$

Then, incident and scattered fields are separated and the latter is expressed by means of the waveguide Green's function

$$
\vec{n} \times\left(\underline{\vec{H}}^{(1)}-\underline{\vec{H}}^{(2)}\right)=-\vec{n} \times \int_{\text {aperture }}\left(G_{H M}^{(1)}+G_{H M}^{(2)}\right) \cdot \underline{\vec{M}} d S,
$$

where $G_{H M}^{(k)}$ is Green's function of waveguide $k$ relating a magnetic source (tangential electric field) with the magnetic field. $\underline{\vec{M}}=\vec{n} \times \underline{\vec{E}}$ 

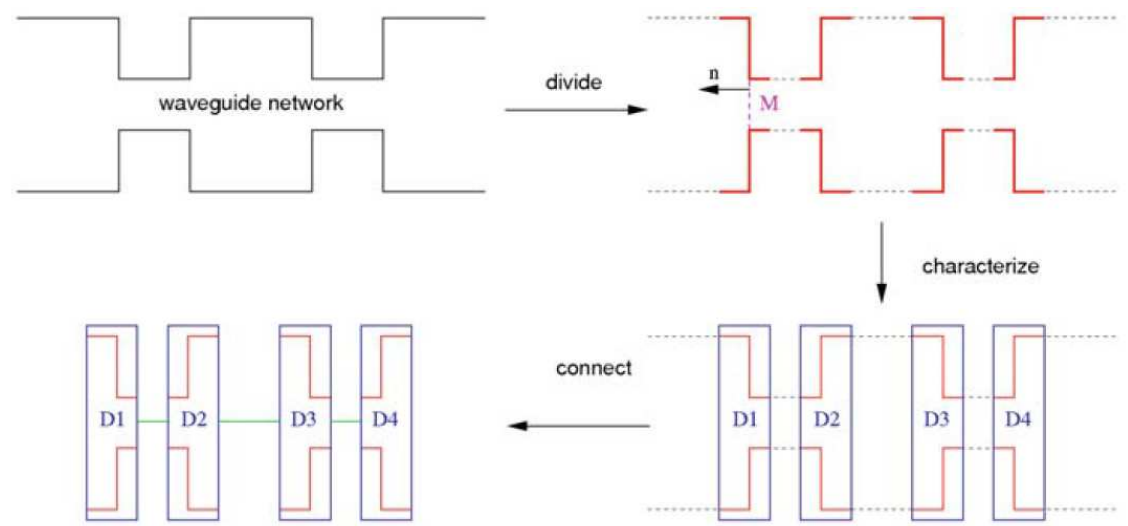

Figure 4.9. Divide-and-conquer approach to model complex devices based on waveguides. D1-D4 are the four discontinuities between the five waveguides. $\underline{\vec{M}}=\vec{n} \times \underline{\vec{E}}$ denotes the tangential electric field in the aperture and is used to set up an integral equation to characterize the discontinuity

is the tangential electric field in the aperture (see Fig. 4.9 upper right picture).

The integral equation (4.21) is solved by means of the method of moments expanding $\underline{\vec{M}}$ with an appropriate basis and testing (4.21) with suitable test functions.

The impedance matrix of each discontinuity is then connected by transmission lines representing the waveguide between two transitions, to obtain the frequency response of the device.

To compute the electromagnetic field, it is expressed using the eigensolutions (modes) associated with the waveguides. For example, the electric field can be written as (see, e.g. [Mar51])

$$
\underline{\vec{E}}(\vec{r}, \omega)=\sum_{k=1}^{\infty} V_{k}(\omega) \cdot \vec{e}_{k}(\vec{r}),
$$

where $\vec{e}_{k}(\vec{r})$ is the $k$ th mode of the electric field of the waveguide and $V_{k}(\omega)$ its amplitude, available after having simulated the response of the device. Similar expression holds for the magnetic field. Equation (4.22) is the field distribution for a harmonic exci- 
4.2. RF breakdown prediction for microwave passive components in multi-carrier operation

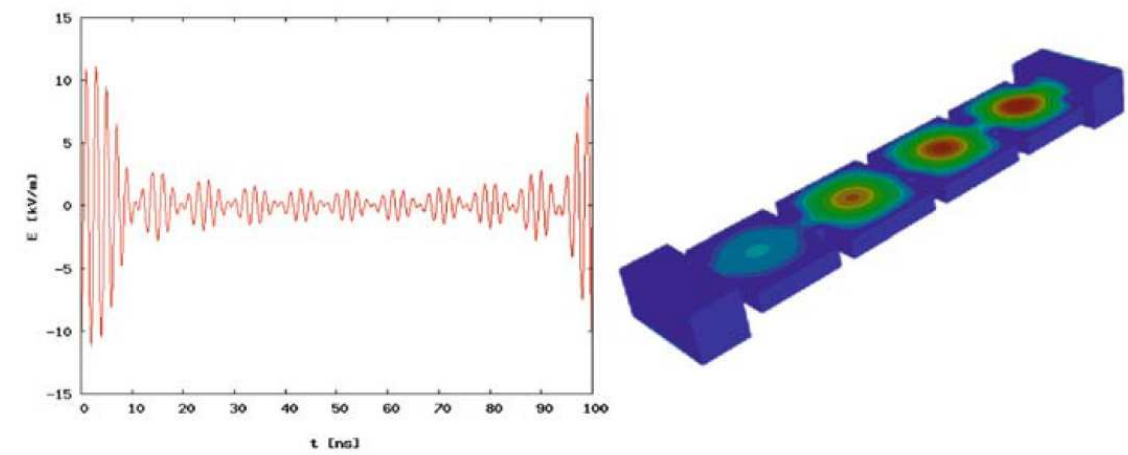

Figure 4.10. Multi-carrier signal: The left picture shows the electric field strength in the centre of one of the cavities and the right figure visualizes the normal component of the electric field on the walls of the band-pass filter

tation of angular frequency $\omega$. For a multicarrier signal, the field distribution is obtained by the superposition principle, i.e. summing the field distribution associated with the individual carriers

$$
\underline{\vec{E}}\left(\vec{r}, \omega_{1}, \ldots, \omega_{n}\right)=\sum_{l=1}^{n} \underline{\vec{E}}\left(\vec{r}, \omega_{l}\right)
$$

$n$ being the number of carriers. Fig. 4.10 shows an example of a multi-carrier signal inside a band-pass filter obtained with the method outline here above.

\subsubsection{Electron Motion and Space Charge Computation}

The diagram of the implemented PIC method is depicted in Fig. 4.11.

Assuming that the initial position and velocity of each electron is known, as well as the initial electric and magnetic fields, the PIC procedure can be summarized as follows (more details on PIC codes can be found, e.g. in [BL05]):

- Particle-to-grid weighting: The charge, position and velocity of each particle are used to interpolate the charge and current densities at the mesh nodes. 


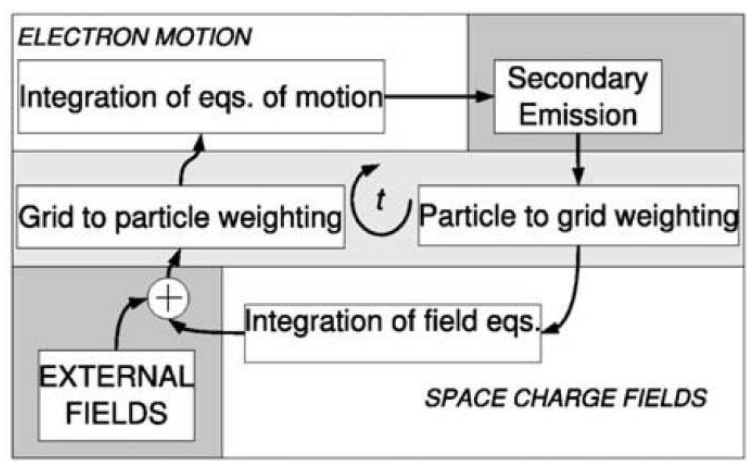

Figure 4.11.

PIC general scheme.

- Integration of field equations: The values of the electric and magnetic fields are computed for the next time step using FDTD of the Maxwell equations.

- External fields: Calculated with the method of Section 3 are added to the space charge fields in order to calculate the total force on the particles.

- Grid-to-particle weighting: The fields at the mesh nodes' locations are interpolated to each one of the particle locations.

- Integration of the equations of motion: The next time step position and velocity are computed employing a leap frog algorithm.

- Secondary Electron Emission (SEE): The SEY is modelled by a modification of the Vaughan's model $\left[\mathrm{VMW}^{+} 05 \mathrm{~b}\right]$.

\subsubsection{Results}

The presented method outlined above has been implemented in a software $\left[\mathrm{AVR}^{+} 08, \mathrm{FES}\right]$. In a first step, simple rectangular waveguides have been used to asses the tool, since, only for these structures, results can be found in literature. Moreover, comparisons with the traditional parallel plate case are possible for which a lot of data are also available.

Two kinds of simulation have been carried out: First, the results of a single-event multipactor discharge are presented and, next, an 
4.2. RF breakdown prediction for microwave passive components in multi-carrier operation

Table 4.4. Frequency and phases of carriers

\begin{tabular}{ccccccccccc}
\hline $\begin{array}{c}\text { Freq. } \\
\text { (GHz) }\end{array}$ & 9.46 & 9.58 & 9.70 & 9.82 & 9.94 & 10.06 & 10.18 & 10.30 & 10.42 & 10.54 \\
$\begin{array}{c}\text { Phase } \\
\left({ }^{\circ}\right)\end{array}$ & 0 & 0 & 0 & 0 & 0 & 0 & 0 & 0 & 0 & 0 \\
\hline
\end{tabular}
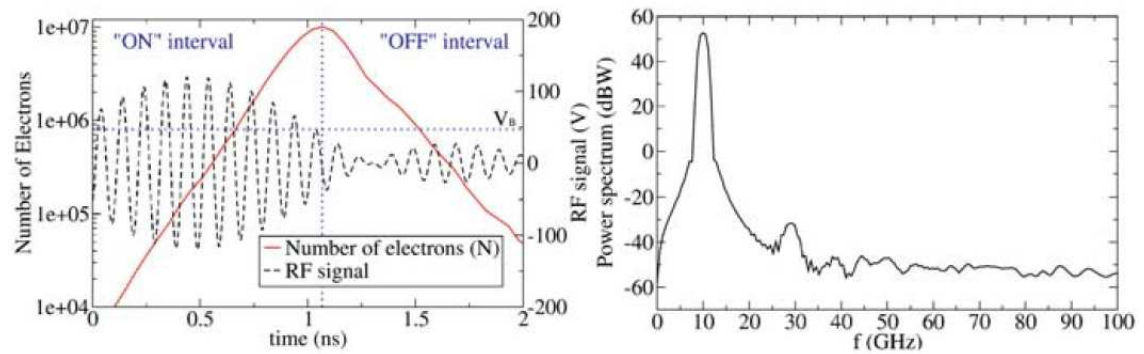

Figure 4.12. RF signal and the number of electrons evolution in time (left) and power spectrum of the fields at output port (right)

inter-period charge accumulation process simulation, which ends up in a long-term multipactor discharge $\left[\mathrm{AVG}^{+} 07\right]$, is shown.

For the single event simulations, a multi-carrier signal composed of 10 carriers of equal amplitude $(12 \mathrm{~V}$ or $16.5 \mathrm{~W})$ was used with the frequencies and phases of Table 4.4, where the voltage is defined as the value of the vertical electric field at the centre plane of the waveguide multiplied by the waveguide height $d$. The resulting signal has a multipactor ON time, $T_{o n}=1 \mathrm{~ns}$. The test has been run on a silver-plated waveguide of dimensions $a=22.86 \mathrm{~mm}, d=0.1 \mathrm{~mm}$, with $f \times d=1 \mathrm{GHz} \cdot \mathrm{mm}$.

The RF applied signal and the evolution of the number of electrons can be seen in Fig. 4.12. As expected, two different behaviours are observed. First, when the envelope is higher than the multipactor threshold $V_{B}$ (47 V from standard susceptibility charts), the number of electrons grows exponentially; next, when the amplitude of the envelope goes below $V_{B}$, the avalanche stops and the electrons start to be absorbed decreasing its population. If the maximum electron density during this period is high enough in order to be detected, we say that a single-event multipactor discharge occurs. In order 
Table 4.5. Frequency and phases of carriers

\begin{tabular}{lcccccccccc}
\hline $\begin{array}{c}\text { Freq. } \\
\text { (GHz) }\end{array}$ & 9.46 & 9.58 & 9.70 & 9.82 & 9.94 & 10.06 & 10.18 & 10.30 & 10.42 & 10.54 \\
$\begin{array}{c}\text { Phase } \\
\left.{ }^{\circ}\right)\end{array}$ & 0 & 41.02 & 82.04 & 123.06 & 164.08 & 164.08 & 123.06 & 82.04 & 41.02 & 0 \\
\hline
\end{tabular}
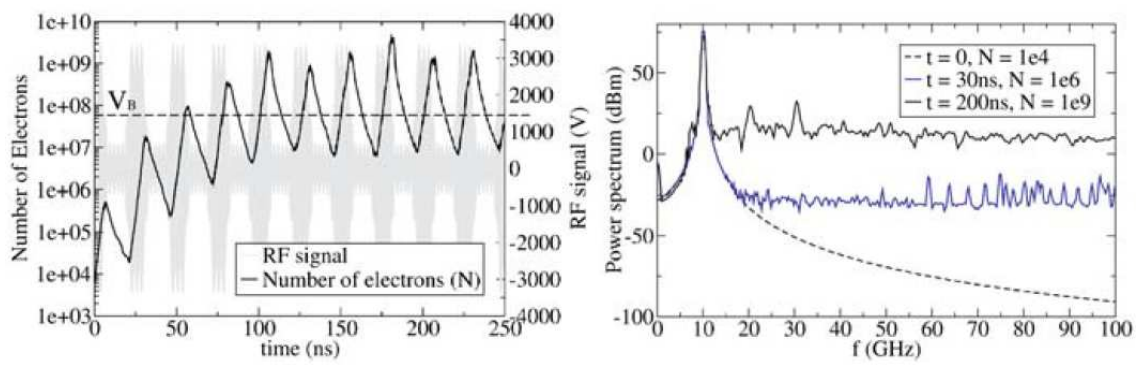

Figure 4.13. RF signal and the number of electrons evolution in time (left) and power spectrum of the signal for three different stages of the discharge (right)

to determine if such number of electrons are significant, the power spectrum of the signal was computed, which is shown in Fig. 4.12. It can be seen, in fact, the presence of the third harmonic generated by the discharge $\left[\mathrm{SAG}^{+} 08\right]$, located to the right of the $\mathrm{RF}$ carrier at a frequency of $30 \mathrm{GHz}$ having an amplitude of about $-30 \mathrm{dBm}$. Considering a typical sensitivity of $-60 \mathrm{dBm}$ for a multipactor experimental set-up [WJ89], this discharge would be quite well detected in the laboratory.

Table 4.5 shows the phase distribution for the long-term discharge simulation. In this case, a waveguide of dimensions $a=22.86 \mathrm{~mm}$, $d=2 \mathrm{~mm}$, with $f \times d=20 \mathrm{GHz} \mathrm{mm}$ and $n=15$, has been chosen. The carriers have equal amplitude of $600 \mathrm{~V}$ each $(2 \mathrm{~kW})$ and follow a triangular distribution which assures enough width and flatness of the main lobe of the multi-carrier envelope to ensure accumulation $\left[\mathrm{AVG}^{+} 07\right]$.

It is important to remark that this specific case ensures a $T_{o n}$ time lower than the time $T_{20}\left(T_{o n}=12 \mathrm{~ns}\right.$ against $\left.T_{20}=15 \mathrm{~ns}\right)$ and that, according to the standards, there should not be a multipactor 
4.2. RF breakdown prediction for microwave passive components in multi-carrier operation

discharge. However, as shown in Fig. 4.13, in this case inter-period accumulation makes the electron population rise to the saturation level producing a multipactor discharge.

The power spectrum of the signal at three different time instants of the discharge is also presented in Fig. 4.13. At initial stages of the discharge, the number of electrons is so low that the harmonics, if present, are not noticeable. But for longer times and higher number of electrons the noise level increases until the third harmonic appears once after reaching saturation.

\subsubsection{Conclusions}

A novel tool for multipactor analysis in passive microwave structures using multi-carrier signals has been presented. It implements a fast and accurate electromagnetic field using a divide-and-conquer strategy. The electron avalanche for multipactor simulations is modelled with a PIC-FDTD code allowing for monitoring the electronic charge density time evolution and the transmitted power spectrum of a multipactor discharge. The simulation results show that discharges also can occur when inter-period discharges are present.

Future enhancements of such a tool, combined with experimental evidences, may help to define a multipactor criterion for multi-carrier signals in order to cover a wide range of cases. 


\subsection{Non-stationary Statistical Theory for Multipactor}

\subsubsection{Introduction}

Multipactor [Far34, GvE48, Vau88] is a resonant nonlinear effect that may occur in high power microwave devices at very low pressures, such as those operating in particle accelerators and satellite subsystems. A multipactor discharge is an electron avalanche in which electrons are accelerated by the electromagnetic fields, successively impacting against the device walls in synchronism with the rf signal frequency. After each impact electrons are released due to secondary emission on the device walls, developing an exponential charge growth and ultimately an electron discharge. Its effects range from signal degradation to the complete destruction of the component. As a consequence, the design of multipactor-free components is a key issue for the space telecommunications industry.

The classical Multipactor theory was first developed by Gill and Von Engel in 1948 [GvE48]. Since then, the theory has evolved in two main branches. The constant- $k$ theory [GvE48,HW54,HW58] and the constant- $v$ theory [Wac64, Vau88]. The former assumes that the emission energy of the secondary electrons is proportional to their impact energy with the proportionality constant $k$. The latter assumes that the emission energy of the secondary electrons is independent from the impact energy. Both the theories are restricted to single carrier continuous wave $(\mathrm{CW})$ operation (the rf signal is composed of only one sinusoid), one dimensional electron motion, parallel plates geometry (homogeneous electrostatic field) and a deterministic energy emission of secondary electrons.

It is known that the emission velocity of secondary electrons does not depend on the primary impact energy [FP02], which is contrary to the constant- $k$ assumption. However, the Secondary Emission Yield (SEY) parameters and the $k$ constant can be easily tuned to adjust the predicted breakdowns to the experimental results [WJ89]. Therefore, the space industry has adopted this theory for the past years and has applied it to the current multipactor standards [EST03].

On the other hand, although the constant- $v$ theory fails to provide reliable breakdown levels [WJ89], it is aligned with the physics 
of secondary emission. For this reason, the constant- $v$ theory has prevailed in the scientific community, and more recent works have extended it in order to cover single surface multipactor with magnetic transversal fields [RCD95] and dielectrics [KLA ${ }^{+}$98], more complicated resonances such as even and hybrid modes [Gil92, $\left.\mathrm{KBS}^{+} 02\right]$ and more complex geometries, such as irises $\left[\mathrm{SRU}^{+} 08\right]$, rectangular waveguides $\left[\mathrm{SRA}^{+} 07\right]$ and coaxial structures [Woo68]. However, the core of these developments remains that of the classical theory and therefore, they fail to predict experimental multipactor breakdown levels.

Recent studies on statistical multipactor describe a more realistic scenario where the random nature of the electron emission velocity is considered [VSS04, VSSS06, SSV08]. In fact, if the velocity spread becomes large enough, the flight time of electrons can strongly vary from impact to impact, even intercalated with single surface impacts, and still have an overall SEY higher than one. This description of the multipactor phenomenon is known as non-resonant multipactor or polyphase multipactor, giving results which are closer to the experiments [VSS04, $\left.\mathrm{SBS}^{+} 05, \mathrm{KLS}^{+} 08\right]$.

This work presents a new non-stationary statistical multipactor theory for single carrier operation, based on the previous work of Vdovicheva et al. [VSS04]. The non-stationary theory accurately predicts multipactor breakdown levels and models electron multiplication and absorption processes experienced during the multipactor discharge. This theory is valid for both stationary and non-stationary situations, giving a more general and reliable theory to explain the complete multipactor physical process.

First, in order to depict a general view, the status and main limitations of the current multipactor theories are briefly summarized in section 4.3.2. Then, the new non-stationary theory is presented in section 4.3.3 and finally verified with numerical and experimental results in sections 4.3.4 and 4.3.5.

\subsubsection{Limitations of the current theories}

Among the classical theories, the multipactor breakdown levels are typically represented through the multipactor susceptibility curves, firstly introduced by Hatch and Williams [HW54]. These curves spec- 
ify the combination of applied voltage, rf frequency $(f)$ and separation between plates or gap $(d)$, needed to start a multipactor discharge. The curves are calculated using the 1D analytical equations of motion of an electron and imposing the conditions of resonance and impact energy, in order to ensure a SEY higher than one (and thus an electron exponential growth). The kind of resonance is set by the multipactor order (or mode), which specifies the number of semi-periods (or cycles) between consecutive electron impacts. The result is a voltage versus $f \times d$ plot, where the regions of multipactor breakdown are delimited for each multipactor order. The susceptibility curves are specific for each material and its respective SEY curve (the SEY depends in turn on the electron impact energy).

\section{Figure 4.14.}

(Color online) Comparison of constant- $k$ and constant- $v$ plots for $k=$ 5 , electron emission energy $W_{0}=1.5 \mathrm{eV}$, and first and second crossover energies (energy at which the SEY is equal to 1) $W_{1}=30$ $\mathrm{eV}$ and $W_{2}=2000$, respectively. Experimental results for silver coating [WJ89] are included.

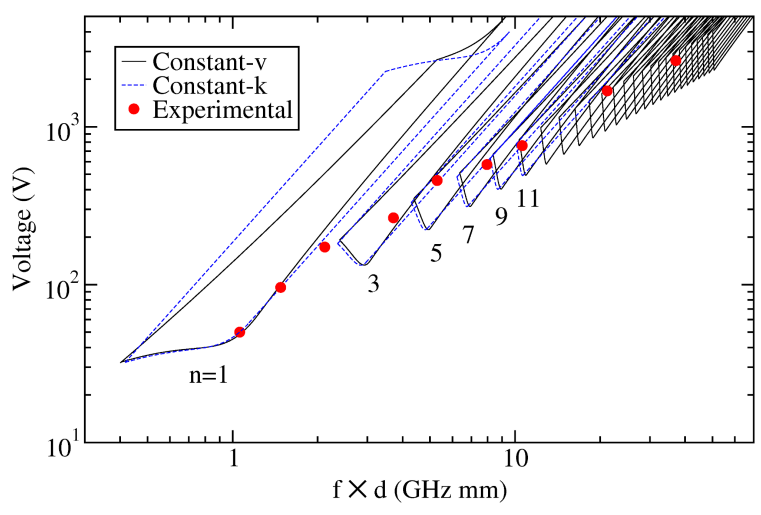

Fig. 4.14 shows an example of multipactor susceptibility curves for silver of Ref. [EST03] whose SEY curve is shown in Fig. 4.15. The susceptibility curves are similar for the constant- $k$ and constant$v$ theories, but they considerably differ for the upper voltage limit of the multipactor zones. The experimental breakdown levels of report [WJ89] are superimposed on the susceptibility curves. Whereas breakdown levels are well predicted for multipactor order $n=1$ (first three experimental points), for higher orders, breakdown levels are underestimated. Therefore, although the constant- $v$ theory is widely accepted as the real physical explanation of multipactor phenomenon, it does not provide accurate multipactor susceptibility curves. This 
is not the case for the constant- $k$ theory, since the aforementioned parameter fitting allows for independently shifting each multipactor order region in order to perfectly match experimental results [WJ89].

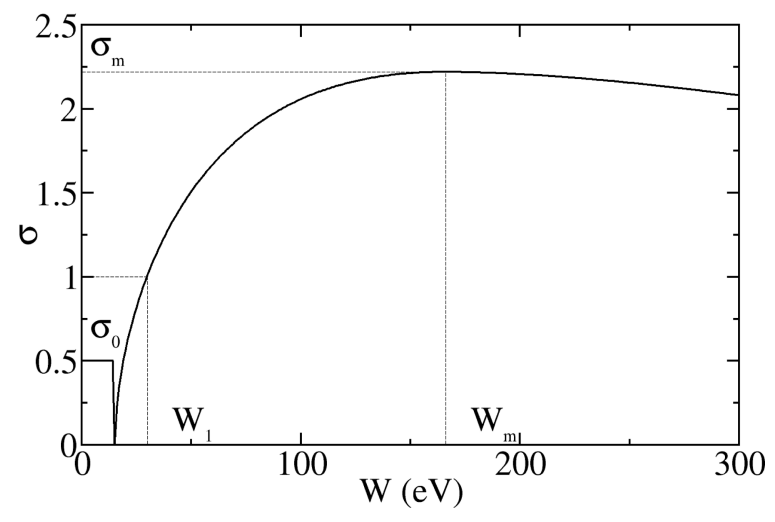

Figure 4.15.

(Color online) SEY curve of silver with parameters $W_{1}=30$ $\mathrm{eV}, W_{m}=165 \mathrm{eV}$, $\sigma_{m}=2.22$ and $\sigma_{0}=0.5$, where $\mathrm{eW}=\frac{1}{2} m_{e} v^{2}$ and $v$ is the electron impact velocity. It uses the parametric model of Vaughan [Vau89] modified by Vicente $\quad\left[\mathrm{VMW}^{+} 05 \mathrm{a}\right]$.

However, even if the constant- $k$ theory is able to match the experimental results through the parameter fitting, it has been severely criticized by some authors [KLA $\left.{ }^{+} 98\right]$, due to its apparently lack of physical basis. Moreover, it seems rather contradictory that a prediction method rely on previous experimental results.

In addition to this, the classical theory models the electron growth for a specific multipactor order as

$$
N(t)=N_{0} \sigma\left(f d, V_{0}\right)^{\frac{2 f t}{n}},
$$

where $N_{0}, \sigma\left(f d, V_{0}\right)$ and $n$ are the initial number of electrons, the SEY and the multipactor order, respectively, for a specific $f \times d$ product and applied voltage $V_{0}$.

In Fig.4.16, a band structure of the SEY values can be observed. This implies that there are some regions where the value of the SEY is much higher than one at the breakdown boundary. For instance, for a $f \times d=2 \mathrm{GHzmm}$, the SEY value at the breakdown boundary would be close to 2 . According to (4.24), this leads to a sudden and discontinuous change in the behavior of the electron growth in the neighborhood of the boundary. This seems quite unrealistic since one would expect a SEY equal to one at the boundaries (neither growth 
nor absorption) and a progressive increment of it as the boundary is exceeded.

\section{Figure 4.16.}

Constant- $v$ multipactor chart for the first eleven modes and energies $W_{0}=1.5 \mathrm{eV}, W_{1}=30$ $\mathrm{eV}$ and $W_{2}=2000 \mathrm{eV}$. The dotted lines represent the contour plot for the different values of the SEY according to the electron impact energies.

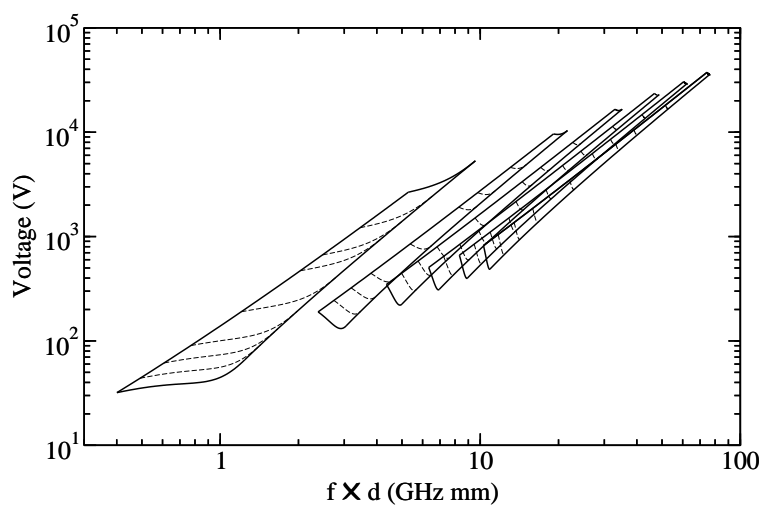

Furthermore, whilst the experimental measurements show a smooth variation of voltage versus $f \times d$, constant- $v$ curves (also constant- $k$ ones but in a lesser extent) present discontinuities between regions of different multipactor modes. This effect is even higher if the stability condition is also considered, which restricts the multipactor regions to the stable phases where the phase-focusing mechanism is enabled [Vau88, Gi195]. Different explanations have arisen for this mismatching. One of those claims that the presence of hybrid modes "fill" the discontinuities between the main modes [Gil92, Kry02].

Later, Vdovicheva et al. proposed a new statistical theory for multipactor [VSS04] which is also restricted to single carrier and parallel plates. But, for the first time, this theory models the random nature of the emission energy of secondary electrons. Through the statistical theory, the authors justify the broadening, overlapping and suppression of the multipactor regions, and the differences between the classical theory and the experimental results. Subsequently, the statistical theory was extended to study the influence of angular anisotropy electron emission velocity [VSSS06] and then particularized for the electromagnetic field distribution of a rectangular waveguide [SSV08].

However, the main limitation of the statistical theory is the assumption of stationarity [VSS04]. Stationarity implies that only the electrons being emitted with a certain emission phase distribution ex- 
perience an exponential growth. As a consequence, as time increases, such distribution is favored over the others, the statistics of the multipactor process converging to a stationary solution. Therefore, the statistics of such a situation can be studied within one single period of the rf signal. Unfortunately, the stationarity assumption limits the validity of the results to the regions in which there is electron exponential growth (above the multipactor breakdown level). Hence, the current statistical theory does not model the electron absorption process which occurs below the multipactor breakdown level. This can be of great interest in some applications in which the rf signal presents a nonstationary behavior, such as multicarrier operation $\left[\mathrm{AVG}^{+} 07\right]$, or modulated signal transmission [ZGPdD03]. Moreover, the stationary theory is only able to delimit the multipactor breakdown regions but does not provide information on the rate of electron growth in time.

Finally, the stationary theory only takes into account double surface interaction. It completely neglects single surface impacts, which indeed may represent a high fraction of the total impacts in the multipactor discharge for increasing voltages above the breakdown level. As a consequence, the stationary theory does not provide a full framework for polyphase multipactor.

\subsubsection{Non-stationary Statistical Multipactor}

The non-stationary statistical theory presented in the following covers the multipactor phenomenon for one-dimensional, parallel plates, electrostatic problems. It is based on the previous and original work on statistical multipactor by Vdovicheva et al. [VSS04].

The key point in the statistical theory (stationary and non-stationary) is to assume that the initial velocity of secondary electrons is a random variable, instead of the deterministic value of the classical theory. This simple difference implies a change of paradigm. In the classical theory, an electron in resonance with the electric field would always start with the same phase relative to the field. It would reach the opposite plate in the same transit time, and would be released again with the same starting phase, repeating the trajectory indefinitely in time. On the contrary, if the emission velocity is a random variable, each time an electron is released, its velocity and corre- 
sponding transit time will be different, according to its probability density function.

Therefore, within the statistical theory it makes no sense to talk about absolute (and deterministic) resonant phases, multipactor orders or impact energies, but rather about probabilities: The probability of an electron to be emitted at a certain phase, probability of an electron to reach the opposite plate in a certain transit time or probability to impact with a certain energy.

The main difference between the stationary and this new nonstationary theory is that the latter does not impose stationarity and, therefore, overcomes the current limitations of the former. As a result, the non-stationary theory is valid for all multipactor regions (exponential growth and absorption) and models both double and single-surface impacts.

\subsubsection{Equations of motion}

First, we start from the one-dimensional equations of motion of the classical theory. Let us consider the motion of an electron with charge $-e$ and mass $m_{e}$ between two infinite parallel plates located at $x=0$ and $x=d$. The sinusoidal electric field of $\mathrm{rf}$ frequency $f$ is $E=$ $-E_{0} \sin (\omega t)$, where $\omega=2 \pi f$. Notice that, for notation purposes, a negative sign has been added to the electric field expression in order to have a positive acceleration. This yields a one-dimensional problem where the electron is accelerated by the Lorentz force, which is equal to $-e E$. The electron maximum speed is considered to be low enough to neglect relativistic effects and, therefore, the acceleration exerted on the electron is given by

$$
m_{e} \ddot{x}=e E_{0} \sin (\omega t)
$$

where the rf voltage $V_{0}$ can be expressed as $V_{0}=E_{0} d$.

The initial conditions at starting time $t=t_{s}$ are $\left.x\right|_{t=t_{s}}=0$ and $\left.\dot{x}\right|_{t=t_{s}}=v_{0}$. From (4.25) the rest of the equations of motion can be derived. Concretely, the position of the electron is

$$
x=\left[v_{0}+v_{\omega} \cos \left(\omega t_{s}\right)\right]\left(t-t_{s}\right)+\frac{v_{\omega}}{\omega}\left[\sin \left(\omega t_{s}\right)-\sin (\omega t)\right],
$$

where $v_{\omega}=e V_{o} /(m \omega d)$. 
In order to work with dimensionless variables, the equation (4.26) is normalized dividing by $v_{\omega} / \omega$. The following notation is used

$$
\begin{gathered}
\xi=\omega x / v_{\omega}, \quad \varphi=\omega t, \quad \varphi_{s}=\omega t_{s}, \\
\varphi_{i}=\omega t_{i}, \quad u=v_{0} / v_{\omega}, \quad \lambda=\omega d / v_{\omega},
\end{gathered}
$$

yielding

$$
\xi\left(\varphi, \varphi_{s}, u\right)=\left(u+\cos \varphi_{s}\right)\left(\varphi-\varphi_{s}\right)+\sin \varphi_{s}-\sin \varphi .
$$

The phase $\varphi_{i}=\omega t_{i}$ at which the electron impacts with the opposite side is the least root of equation (4.28) setting the normalized electron position equal to the normalized gap, i.e $\xi=\lambda$. This yields

$$
\lambda=\left(u+\cos \varphi_{s}\right)\left(\varphi_{i}-\varphi_{s}\right)+\sin \varphi_{s}-\sin \varphi_{i},
$$

Note that the equation (4.29) is derived from the classical constant$v$ theory and establishes the condition for an electron to reach the opposite plate, $\xi=\lambda$ at impacting phase $\varphi_{i}$, with starting phase $\varphi_{s}$, and initial velocity $u$.

\subsubsection{Definitions and conventions}

Contrarily to the stationary counterpart, the non-stationary theory is not restricted to the study of the probabilities over a period of the rf signal, but rather extends the time window from $t=0$ to infinity. It imposes initial conditions and calculates the time evolution of the different probabilities indefinitely in time. Let as denote each plate as " $D$ " and " $U$ " for the boundary conditions $\xi=0$ (Down) and $\xi=\lambda(\mathrm{Up})$, respectively. Thus two kinds of interactions are considered: double surface ( $D-U$ or $U-D$ trajectories) and single surface ( $D-D$ and $U-U$ trajectories). All their related parameters are properly labelled as " $d s$ " and "ss", respectively. Fig. 4.17 depicts these concepts.

Table 4.6 introduces the most relevant definitions of the nonstationary theory which constitute the basis of its development. In the next sections we will derive the analytical expressions for all of them. 
Figure 4.17.

Non-stationary definitions and conventions. Some possible electron trajectories are depicted and labelled with the corresponding type of interaction, double surface (dd) and single surface (ss).

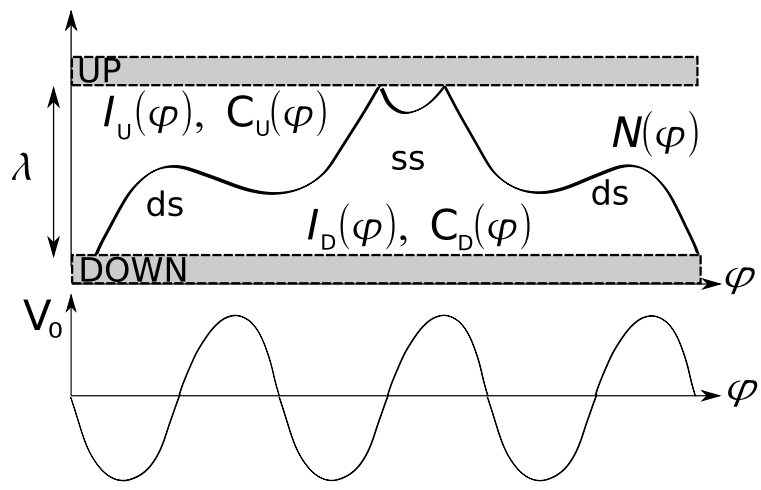

Table 4.6. Non-stationary theory definitions.

\begin{tabular}{lr}
\hline $\begin{array}{l}\text { Impact rate (electrons / radian) in plate } U / D \\
\text { at phase } \varphi\end{array}$ & $I_{U / D}(\varphi)$ \\
\hline Emission rate (electrons / radian) in plate & $C_{U / D}(\varphi)$ \\
$U / D$ at phase $\varphi$ & $N(\varphi)$ \\
\hline Number of electrons at time $\varphi$ & \\
\hline Probability density that an electron starting & \\
at plate $U / D$, with starting phase $\varphi$, expe- & $G_{d s / s s, U / D}(\tau \mid \varphi)$ \\
riences a double/single surface impact in a & \\
transit phase $\tau$ & \\
\hline SEY of an electron starting at plate $U / D$, & \\
with starting phase $\varphi$ which experiences a & $\sigma_{d s / s s, U / D}(\tau \mid \varphi)$ \\
double/single surface impact in a transit \\
phase $\tau$
\end{tabular}

\subsubsection{Statistical development}

The joint probability density $G\left(\tau \mid \varphi_{s} ; \lambda\right)$ is defined by Vdovicheva et al. as the probability that an electron released at phase $\varphi_{s}$ impacts with the opposite wall, separated by $\lambda$, in a transit phase $\tau$ [VSS04].

In order to construct $G\left(\tau \mid \varphi_{s} ; \lambda\right)$ one departs from the known probability density function of the electron emission velocity $u$, namely $f_{u}(u)$. According to the theorem of transformation of Univariate Random Variables, of the statistical theory, $G\left(\tau \mid \varphi_{s} ; \lambda\right)$ can be writ- 
ten as

$$
G\left(\tau \mid \varphi_{s} ; \lambda\right)=\left|\frac{d g\left(\tau \mid \varphi_{s} ; \lambda\right)}{d \tau}\right| f_{u}\left(g\left(\tau \mid \varphi_{s} ; \lambda\right)\right),
$$

where $u=g\left(\tau \mid \varphi_{s} ; \lambda\right)$ must be a monotonic function which expresses $u$ as a function of $\tau$.

From (4.29), $u$ can be easily worked out, obtaining a candidate function $g_{0}\left(\tau \mid \varphi_{s} ; \lambda\right)$.

$$
u=g_{0}\left(\tau \mid \varphi_{s} ; \lambda\right)=\frac{\lambda-\sin \varphi_{s}+\sin \left(\varphi_{s}+\tau\right)}{\tau}-\cos \varphi_{s},
$$

being $\tau=\varphi_{i}-\varphi_{s}$.

By definition, $g$ must be monotonic with $\tau$. But, unfortunately, the candidate $g_{0}$ is a non-monotonic function due to its sinusoidal behavior. The non-monotonicity of $g_{0}$ denotes many solutions of $\tau$ for a single release velocity $u$. This implies that the trajectory of an electron crosses the boundary $\xi=\lambda$ more than once, which is physically impossible (each crossing implies an impact and there cannot be more than one for a trajectory). Therefore, the non-monotonicity of $g_{0}$ can (and must) be removed to obtain $g$ and solve (4.30). This forces $G\left(\tau \mid \varphi_{s} ; \lambda\right)$ to be 0 in the non-monotonic intervals of $g_{0}$. In addition, $g$ must be greater than a minimum starting velocity (greater than 0) to reach the opposite plate. Vdovicheva et al. gives a detailed derivation of $G\left(\tau \mid \varphi_{s} ; \lambda\right)$ and such minimum velocity [VSS04].

The function $G\left(\tau \mid \varphi_{s} ; \lambda\right)$ is the basis of the statistical theory. From it, Vdovicheva et al. formulate the problem by imposing stationarity and find the multipactor breakdown boundaries by solving a homogeneous Fredholm integral equation of the second kind [VSS04].

The non-stationary theory presented in this work also starts from $G\left(\tau \mid \varphi_{s} ; \lambda\right)$, but follows a different approach, which is detailed next. First, we introduce another definition. $G\left(\tau \mid \varphi_{s} ; 0\right)$ is the probability that an electron released at phase $\varphi_{s}$ impacts back to the emission surface in a transit time $\tau$ (single surface impact). It is constructed in a similar way to (4.30).

$$
G\left(\tau \mid \varphi_{s} ; 0\right)=\left|\frac{d g\left(\tau \mid \varphi_{s} ; 0\right)}{d \tau}\right| f_{u}\left(g\left(\tau \mid \varphi_{s} ; 0\right)\right),
$$

where $u=g\left(\tau \mid \varphi_{s} ; 0\right)$ is derived applying $\xi=0$ at impact time $\varphi_{i}=\omega t_{i}$ to $(4.28)$, and working out the velocity $u$. This yields the 
candidate function

$$
u=g_{0}\left(\tau \mid \varphi_{s} ; 0\right)=\frac{-\sin \varphi_{s}+\sin \left(\varphi_{s}+\tau\right)}{\tau}-\cos \varphi_{s} .
$$

The $g\left(\tau \mid \varphi_{s} ; 0\right)$ function can be obtained removing the non-monotonic intervals of (4.33). The minimum ejection velocity for double surface is equal to the maximum ejection velocity for single surface, i.e. $\min \left(u_{d s}\right)=\max \left(u_{s s}\right)$, where $u_{d s} \equiv g\left(\tau \mid \varphi_{s} ; \lambda\right)$ and $u_{s s} \equiv g\left(\tau \mid \varphi_{s} ; 0\right)$.

Fig. 4.18 shows an example of $u_{d s}$ and $u_{s s}$. For this specific case $\min \left(u_{d s}\right)=\max \left(u_{s s}\right)=0.55$. Therefore, if the emission velocity $u$ is higher than 0.55 there will be a double surface impact. For lower emission velocities, the electron will suffer a single surface impact.

\section{Figure 4.18.}

(Color online) Example of $u_{d s}$ and $u_{s s}$ for $V_{0}=$ $120 \mathrm{~V}, f=1.64 \mathrm{GHz}$, $d=1 \mathrm{~mm}$ and $\varphi_{s}=$ $122^{\circ}$.

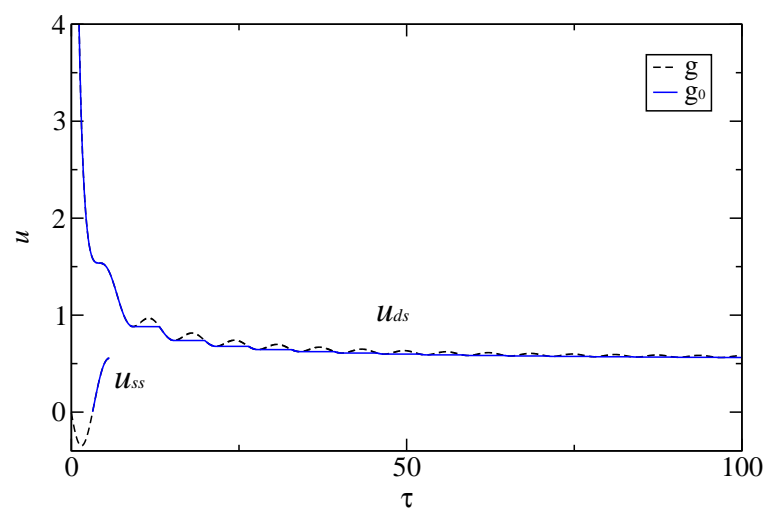

The $G(\tau \mid \varphi)$ functions of Table. 4.6 are defined as

$$
\begin{aligned}
& G_{d s, D}(\tau \mid \varphi)=G\left(\tau \mid \varphi_{s} ; \xi\right) \mid \begin{array}{l}
\xi=\lambda \\
\varphi_{s}=\bmod (\varphi ; 2 \pi)
\end{array} \\
& G_{d s, U}(\tau \mid \varphi)=G\left(\tau \mid \varphi_{s} ; \xi\right) \mid \begin{array}{l}
\xi=\lambda \\
\varphi_{s}=\bmod (\varphi+\pi ; 2 \pi)
\end{array} \\
& G_{s s, D}(\tau \mid \varphi)=G\left(\tau \mid \varphi_{s} ; \xi\right) \mid \begin{array}{l}
\xi=0 \\
\varphi_{s}=\bmod (\varphi ; 2 \pi)
\end{array} \\
& G_{s s, U}(\tau \mid \varphi)=G\left(\tau \mid \varphi_{s} ; \xi\right) \mid \begin{array}{l}
\xi=0 \\
\varphi_{s}=\bmod (\varphi+\pi ; 2 \pi)
\end{array}
\end{aligned}
$$


The $G(\tau \mid \varphi)$ functions are periodic with respect to $\varphi$ with a period of $2 \pi$. Note that the direction of the electron normal emission is reversed for opposite plates, and that the rf field changes sign every $\pi$ radian. Therefore, the $G$ functions for the $U$ and $D$ plates are related by a relative shift of $\pi$ radians.

The double surface interactions imply that the electron crosses the gap, and therefore $\xi=\lambda$, whereas single surface interactions means that the electron impacts back to the emission surface, $\xi=$ 0 . Therefore the subscripts $d s$ or $s s$ indicate that the solution of $G\left(\tau \mid \varphi_{s} ; \xi\right)$ is particular for $\xi=\lambda$ or $\xi=0$, respectively.

The $G_{d s}$ and $G_{s s}$ functions corresponding to the example of Fig. 4.18 are presented in Fig. 4.19. In this case, a Maxwellian distribution for the initial velocity is taken.

$$
f_{u}=\frac{u v_{\omega}^{2}}{v_{t}^{2}} \exp \left(-\frac{u^{2} v_{\omega}^{2}}{2 v_{t}^{2}}\right)
$$

where $v_{t}$ is the velocity thermal spread. A value of $W_{t}=1.5 \mathrm{eV}$ has been taken for this work, where $W_{t}=\frac{m_{e}}{2 e} v_{t}^{2}$ is the thermal emission energy spread. The possible electron transit times are restricted to the regions where the $G_{d s}$ and $G_{s s}$ are not zero. For this specific example, the $G_{s s}$ plot (bottom) shows that the transit time for a single surface impact must lie somewhere in between (approximately) $\tau=\pi$ and $\tau=7 \pi / 4$. In contrast, all double surface impacts (top) have a transit time higher than $\tau=3 \pi / 2$. There is a small overlap of both functions in the interval $[3 \pi / 2-7 \pi / 4]$, which means that the electrons impacting with such a transit time could have suffered either a double surface interaction or a single surface one, with different probability.

The noticeable difference in amplitude comes from the fact that each function obeys

$$
\int_{0}^{\infty} G_{d s}(\tau \mid \varphi) d \tau=P_{d s}(\varphi) \text { and } \int_{0}^{\infty} G_{s s}(\tau \mid \varphi) d \tau=P_{s s}(\varphi),
$$

where $P_{d s}(\varphi)$ and $P_{s s}(\varphi)$ are the probability that an electron emitted at $\varphi$ suffers a double or single surface impact, respectively. Note that $P_{d s}(\varphi)+P_{s s}(\varphi)=1$.

Once the $G(\tau \mid \varphi)$ functions are obtained, the rest of the definitions given in Table 4.6 can be derived in the following manner. Let us 
Figure 4.19.

example of $G_{d s}$ and $G_{s s}$ probability functions for $V_{0}=120 \mathrm{~V}, f=$ $1.64 \mathrm{GHz}, d=1 \mathrm{~mm}$, $\varphi_{s}=122^{\circ}$ and the emission velocity function of (4.35). The detailed window of the lower plot shows the superposition of both functions for the first 3 half-periods of the transit time.
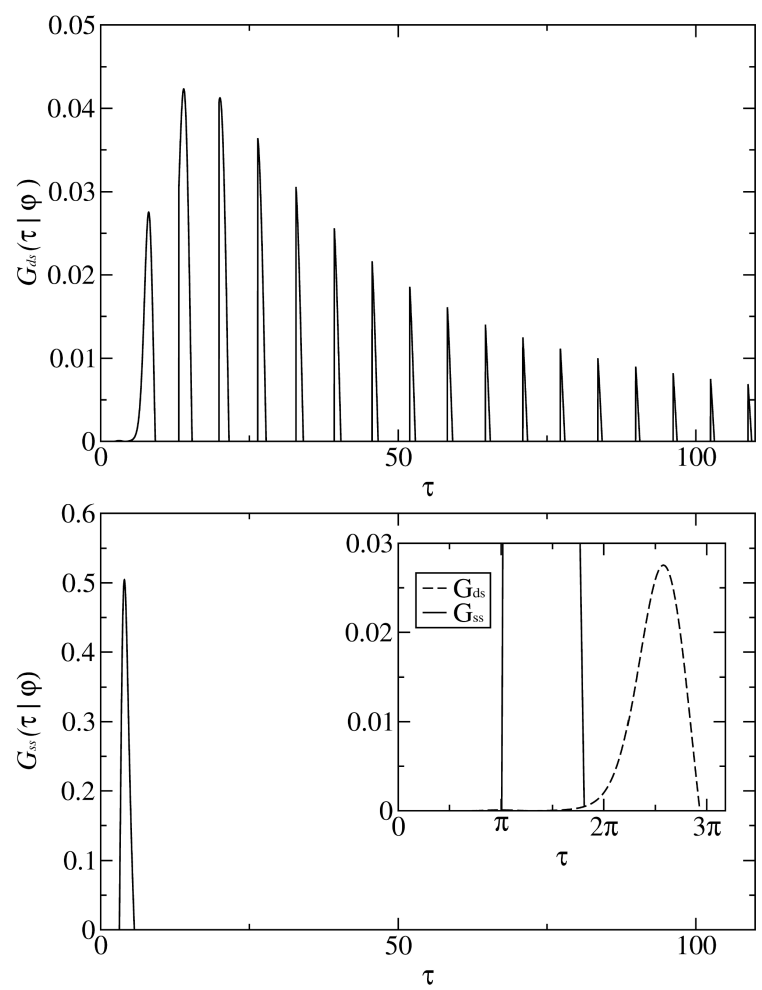

take the rate of emission at the plate $U, C_{U}(\varphi)$. At a certain $\varphi$, the number of impacting electrons is the result of two contributions: the electrons released from the opposite plate, $D$, that followed a double surface interaction, and those emitted from the same plate, $U$, which suffered a single surface interaction (see Fig. 4.20).

The emission rate at phase $\varphi$ due to double surface interaction of an electron emitted at phase $\varphi^{\prime}$ from the plate $D$, where $\varphi^{\prime}<\varphi$, is

$$
C_{D}\left(\varphi^{\prime}\right) G_{d s, D}\left(\varphi-\varphi^{\prime} \mid \varphi^{\prime}\right) \sigma_{d s, D}\left(\varphi-\varphi^{\prime} \mid \varphi^{\prime}\right),
$$

$C_{D}$ being the rate of emission at the plate $D$.

On the other hand, the emission rate at phase $\varphi$ due to single surface interaction of an electron emitted at phase $\varphi^{\prime}$ from the plate $U$, where $\varphi^{\prime}<\varphi$, is

$$
C_{U}\left(\varphi^{\prime}\right) G_{s s, U}\left(\varphi-\varphi^{\prime} \mid \varphi^{\prime}\right) \sigma_{s s, U}\left(\varphi-\varphi^{\prime} \mid \varphi^{\prime}\right) .
$$




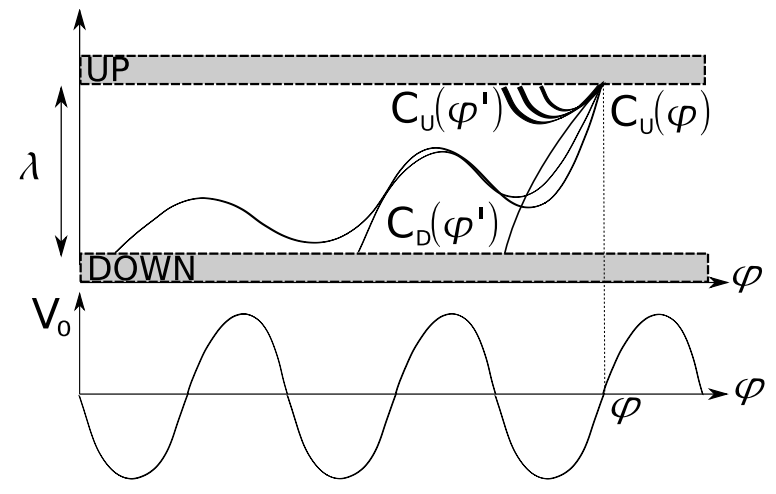

Figure 4.20.

Dependence of $C_{U}(\varphi)$ on the previous instants $\varphi^{\prime}$. There are two contributions to $C_{U}(\varphi)$, electrons emitted from the plate $D\left(C_{D}\left(\varphi^{\prime}\right)\right)$, following a double surface interaction, and electrons emitted from plate $U\left(C_{U}\left(\varphi^{\prime}\right)\right)$, which experience a single surface interaction.
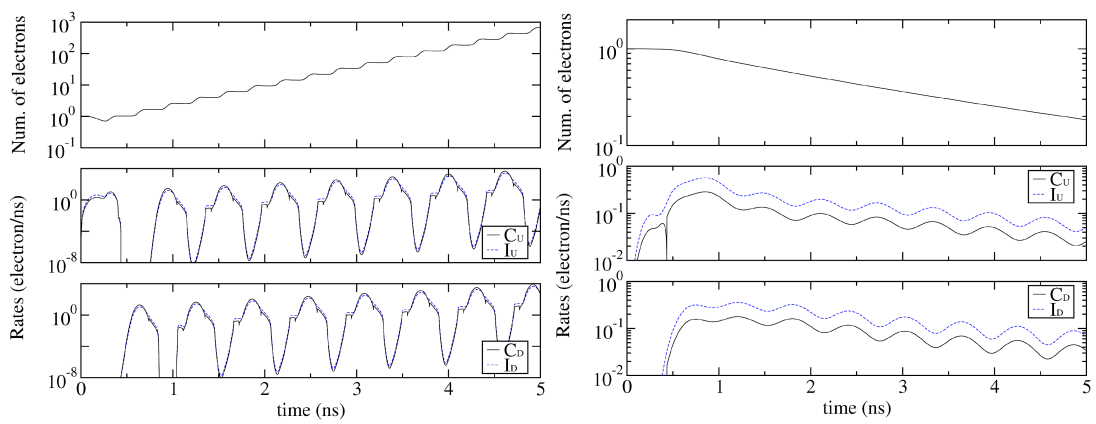

Figure 4.21. (Color online) Time evolution of the electron population and emission and impact rates for both plates for $V_{0}=120 \mathrm{~V}$ (left) and $V_{0}=10 \mathrm{~V}$ (right), with $f=1.64 \mathrm{GHz}$ and $d=1 \mathrm{~mm}$.

Therefore, the total rate of emission at the top plate and instant $\varphi$ is the integration of the previous quantities from the initial instant 
$\varphi^{\prime}=0$ to the current time $\varphi^{\prime}=\varphi$, yielding

$$
\begin{aligned}
& C_{U}(\varphi)= \\
& =\int_{0}^{\varphi} C_{D}\left(\varphi^{\prime}\right) G_{d s, D}\left(\varphi-\varphi^{\prime} \mid \varphi^{\prime}\right) \sigma_{d s, D}\left(\varphi-\varphi^{\prime} \mid \varphi^{\prime}\right) d \varphi^{\prime}+ \\
& +\int_{0}^{\varphi} C_{U}\left(\varphi^{\prime}\right) G_{s s, U}\left(\varphi-\varphi^{\prime} \mid \varphi^{\prime}\right) \sigma_{s s, U}\left(\varphi-\varphi^{\prime} \mid \varphi^{\prime}\right) d \varphi^{\prime}+ \\
& +\Psi_{U}(\varphi),
\end{aligned}
$$

where $\Psi_{U}(\varphi)$ is the external source of electrons from the plate $U$.

The rate of emitted electrons at the plate $D, C_{D}(\varphi)$, can be derived in a similar way yielding

$$
\begin{aligned}
& C_{D}(\varphi)= \\
& =\int_{0}^{\varphi} C_{D}\left(\varphi^{\prime}\right) G_{s s, D}\left(\varphi-\varphi^{\prime} \mid \varphi^{\prime}\right) \sigma_{s s, D}\left(\varphi-\varphi^{\prime} \mid \varphi^{\prime}\right) d \varphi^{\prime}+ \\
& +\int_{0}^{\varphi} C_{U}\left(\varphi^{\prime}\right) G_{d s, U}\left(\varphi-\varphi^{\prime} \mid \varphi^{\prime}\right) \sigma_{d s, U}\left(\varphi-\varphi^{\prime} \mid \varphi^{\prime}\right) d \varphi^{\prime}+ \\
& +\Psi_{D}(\varphi),
\end{aligned}
$$

Equations (4.36) and (4.37) constitute a system of Volterra integral equations of the second kind with difference kernel. This system has a non-trivial solution only when $\Psi_{U}(\varphi)+\Psi_{D}(\varphi)>0$ and $\Psi_{U}(\varphi) \geq 0, \Psi_{D}(\varphi) \geq 0$ (see chapter 9.3 of Ref. [PM98]). These functions set the initial conditions of the problem. From a physical point of view this implies that electron emission is only possible when there is an external source of electrons, which makes sense, since electrons cannot appear "spontaneously".

Analogously to (4.36), the remaining definitions of Table 4.6 can be expressed as follows

$$
\begin{aligned}
& I_{U}(\varphi)=\int_{0}^{\varphi} C_{D}\left(\varphi^{\prime}\right) G_{d s, D}\left(\varphi-\varphi^{\prime} \mid \varphi^{\prime}\right) d \varphi^{\prime}+ \\
& +\int_{0}^{\varphi} C_{U}\left(\varphi^{\prime}\right) G_{s s, U}\left(\varphi-\varphi^{\prime} \mid \varphi^{\prime}\right) d \varphi^{\prime}
\end{aligned}
$$


and

$$
\begin{aligned}
& I_{D}(\varphi)=\int_{0}^{\varphi} C_{D}\left(\varphi^{\prime}\right) G_{s s, D}\left(\varphi-\varphi^{\prime} \mid \varphi^{\prime}\right) d \varphi^{\prime}+ \\
& +\int_{0}^{\varphi} C_{U}\left(\varphi^{\prime}\right) G_{d s, U}\left(\varphi-\varphi^{\prime} \mid \varphi^{\prime}\right) d \varphi^{\prime} .
\end{aligned}
$$

Finally, the number of electrons at time $\varphi$ is the integration over time of all emitted electrons minus the impacting electrons for both $U$ and $D$ plates, i.e.

$$
N(\varphi)=\int_{0}^{\varphi} C_{U}\left(\varphi^{\prime}\right)+C_{D}\left(\varphi^{\prime}\right)-I_{U}\left(\varphi^{\prime}\right)-I_{D}\left(\varphi^{\prime}\right) d \varphi^{\prime} .
$$

\subsubsection{Theoretical Analysis}

For a given gap, $d$, signal parameters $E$ and $f$, SEY function $\sigma$ (characterizing the material), and seeding function $\Psi(\varphi)$, it is possible to compute all above functions. First, $d, E$ and $f$ are substituted in the equations of motion given in (4.27) and (4.28). The $G_{d s}(\tau \mid \varphi)$ and $G_{s s}(\tau \mid \varphi)$ functions are computed using (4.31), (4.30), (4.33) and (4.32). Later, the system of Volterra integral equations constituted by (4.36) and (4.37) is solved (in most cases with standard numerical techniques [PTVF07]) finding the electron emission functions $C_{U}$ and $C_{D}$. Then, $I_{U}$ and $I_{D}$ are obtained by simple integration of (4.38) and (4.39) and finally, $N$ is given by (4.40).

Fig. 4.21 shows an example for the Maxwellian emission velocity distribution given in (4.35). The SEY model is taken from paper $\left[\mathrm{AVG}^{+} 07\right.$ ], with the SEY parameters of silver given in Ref. [EST03]: $\sigma_{0}=0.5, \sigma_{m}=2.22, W_{1}=30 \mathrm{eV}$ and $W_{m}=165 \mathrm{eV}$ (see Fig. 4.15). The external seeding has been set to

$$
\Psi_{U}(\varphi)=\Psi_{D}(\varphi)=\frac{1}{2} \delta(\varphi),
$$

where $\delta(\varphi)$ is the Dirac delta function. This means that seeding electrons are only injected at $\varphi=0$.

In both cases, a repetitive pattern can be appreciated. Notice how, after a transitory interval at the beginning, the curves associated to the $U$ and $D$ plates evolve to adopt a similar shape but shifted in time (exactly $\pi$ radians). The electron population, $N(t)$, is altered 
whenever there is an impact in any of the plates. Therefore, the variation of the electron population curve perfectly agrees with the peaks of the emission and impact curves $\left(C_{U}\right.$ and $C_{D}$ for the former and $I_{U}$ and $I_{D}$ for the latter).

This can be better appreciated in the $V_{0}=120 \mathrm{~V}$ case [Fig. 4.21 (left)] where the rf voltage is above the breakdown level. In general, the rate of electron emission is higher than the electron impact (or absorption), and therefore there is an overall increase of charge with time, following an exponential trend.

The $V_{0}=10 \mathrm{~V}$ case [Fig. 4.21 (right)] shows a situation where the rf voltage is below the breakdown level. In this case the impact curves are always higher than the emission ones and therefore there is an overall destruction of charges. Hence, the electron population decreases in time.

It may be also useful to define SEY and multipactor order parameters equivalently to the classical Theory. The instantaneous SEY is the ratio between the total emission rate divided by the total impact rate, thus

$$
\sigma_{i}(\varphi)=\frac{C_{(\varphi)}}{I(\varphi)},
$$

where $C(\varphi)=C_{U}(\varphi)+C_{D}(\varphi)$ and $I(\varphi)=I_{U}(\varphi)+I_{D}(\varphi)$. On the other hand, the instantaneous order is computed as the statistical expectation of the flight time for all kind of interactions divided by the total impact rate.

$$
\begin{aligned}
n_{i}(\varphi) & =\frac{1}{\pi I(\varphi)} \\
& {\left[\int_{0}^{\varphi}\left(\varphi-\varphi^{\prime}\right) C_{D}\left(\varphi^{\prime}\right) G_{d s, D}\left(\varphi-\varphi^{\prime} \mid \varphi^{\prime}\right) d \varphi^{\prime}+\right.} \\
& +\int_{0}^{\varphi}\left(\varphi-\varphi^{\prime}\right) C_{U}\left(\varphi^{\prime}\right) G_{d s, U}\left(\varphi-\varphi^{\prime} \mid \varphi^{\prime}\right) d \varphi^{\prime}+ \\
& +\int_{0}^{\varphi}\left(\varphi-\varphi^{\prime}\right) C_{D}\left(\varphi^{\prime}\right) G_{s s, D}\left(\varphi-\varphi^{\prime} \mid \varphi^{\prime}\right) d \varphi^{\prime}+ \\
& \left.+\int_{0}^{\varphi}\left(\varphi-\varphi^{\prime}\right) C_{U}\left(\varphi^{\prime}\right) G_{s s, U}\left(\varphi-\varphi^{\prime} \mid \varphi^{\prime}\right) d \varphi^{\prime}\right]
\end{aligned}
$$


Finally, in an interval $\Delta \varphi$, average quantities are defined as

$$
\sigma_{a v}(\varphi)=\frac{\int_{\varphi}^{\varphi+\Delta \varphi} \sigma_{i}\left(\varphi^{\prime}\right) I\left(\varphi^{\prime}\right) d \varphi^{\prime}}{\int_{\varphi}^{\varphi+\Delta \varphi} I\left(\varphi^{\prime}\right) d \varphi^{\prime}}
$$

and

$$
n_{a v}(\varphi)=\frac{\int_{\varphi}^{\varphi+\Delta \varphi} n_{i}\left(\varphi^{\prime}\right) I\left(\varphi^{\prime}\right) d \varphi^{\prime}}{\int_{\varphi}^{\varphi+\Delta \varphi} I\left(\varphi^{\prime}\right) d \varphi^{\prime}}
$$

for the SEY and multipactor order, respectively. These two parameters characterize the electron growth, since, although the exact solution can be found integrating (4.40), an easier and faster approximation for an interval $\Delta t=\frac{\Delta \varphi}{\omega}$ is given by

$$
N(t+\Delta t) \approx N(t) \sigma_{a v}^{\frac{2 f \Delta t}{n_{a v}}} .
$$

\subsubsection{Numerical Simulations}

The numerical results have been obtained with the full-wave electromagnetic solver tool FEST3D $\left[\mathrm{VMW}^{+} 05 \mathrm{a}, \mathrm{AVR}^{+} 08\right]$ which also incorporates a 3D Particle-In-Cell (PIC) multipactor module. FEST3D is able to simulate arbitrary 3D structures with inhomogeneous electromagnetic field distributions. However, in order to compare the results with the present theory, the simulations have been restricted to 1D electron motion and homogeneous electric field. The FEST3D multipactor module computes the electron trajectories, determines all electron impacts (both single and double surface) and records the associated flight time and SEY for each one. The simulations have been done for a number of 50000 initial particles, to have enough samples for a good statistical analysis. During the simulation, the particles can be created or destroyed depending on the impact energy. The results have been normalized to have one initial electron, in order to compare with the analytical results.

The numerical equivalent SEY is the average SEY for all impacts.

$$
\sigma_{e q}=\frac{\sum_{i=1}^{i=N_{t}} \sigma_{i}}{N_{t}}
$$

where $N_{t}$ is the total number of impacts, and $\sigma_{i}$ is the secondary emission yield at impact $i$, respectively. The numerical equivalent 
multipactor order is defined as the average travel time, divided by half period of the rf signal.

$$
n_{e q}=2 f \frac{\sum_{i=1}^{i=N_{t}} t_{i}}{N_{t}}
$$

where $t_{i}$ is the flight time for impact $i$.
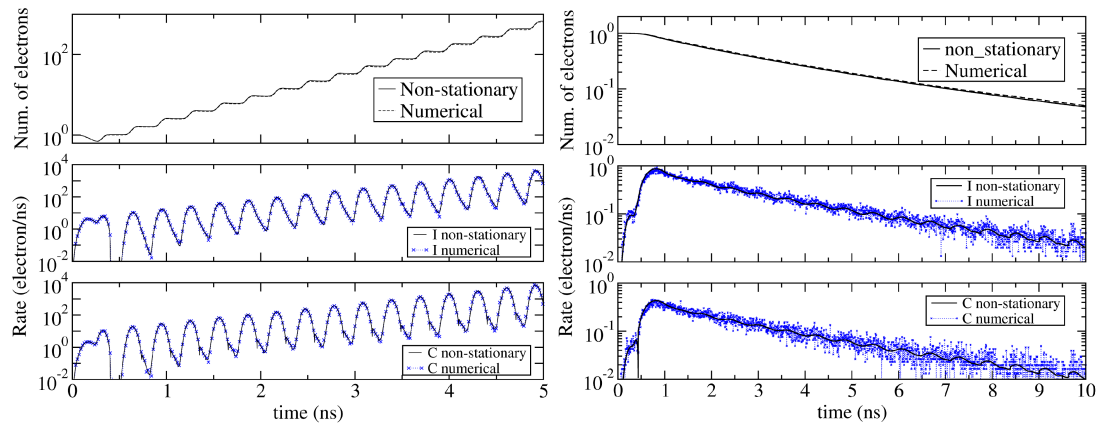

Figure 4.22. (Color online) Time evolution of electron population and emission and absorption rates for $V_{0}=120 \mathrm{~V}$ (left) and $V_{0}=10$ $\mathrm{V}$ (right), with $f=1.64 \mathrm{GHz}$ and $d=1 \mathrm{~mm}$.
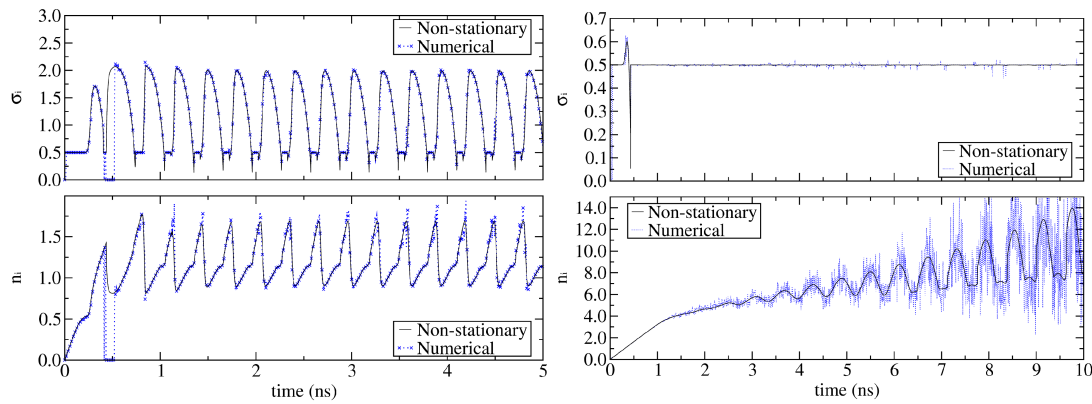

Figure 4.23. (Color online) Electron population and instantaneous SEY and order or $V_{0}=120 \mathrm{~V}$ (left) and $V_{0}=10 \mathrm{~V}$ (right), with $f=1.64 \mathrm{GHz}, d=1 \mathrm{~mm}$ and $\varphi_{s}=122^{\circ}$. 


\subsubsection{Results}

All the simulations and analytical results have been carried out assuming the Maxwellian distribution of (4.35) for the electron initial velocity and $W_{t}=1.5 \mathrm{eV}$. The SEY model is that of Ref. [VMW $\left.{ }^{+} 05 \mathrm{c}\right]$. The SEY parameters are for the silver given in paper [EST03], i.e, $\sigma_{m}=2.22, W_{1}=30 \mathrm{eV}$ and $W_{m}=165 \mathrm{eV}$ with $\sigma_{0}=0.5$ (see Fig. 4.15).

Fig. 4.22 shows the time evolution of a multipactor discharge. Two different scenarios are considered. First, a case with a voltage above the breakdown level in which there is an electron exponential growth (left); and a non-resonant, non-stationary situation below breakdown, in which there is an overall electron absorption (right). The analytical evolution in time of the electron population $N$, total rate of impact $I$ and total rate of electron emission $C$, given by (4.40) and (4.36)-(4.39), respectively, are plotted and compared with numerical simulations, with identical initial conditions. Besides the inherent numerical noise of the simulator due to the limited number of samples (electrons), it can be observed an excellent agreement between numerical simulations and the non-stationary theory.

The instantaneous SEY and order defined by (4.41) and (4.42) have been also compared with numerical simulations in Fig. 4.23. Again, a very good agreement between theory and numerical results is observed. Here, the stationarity of the above-breakdown case is evidenced (left). After an initial transitory, both the SEY and order evolve towards a periodic structure. Nevertheless, in the belowbreakdown situation (right), although the SEY keeps constant (due to the low impact energy of the electrons), the multipactor order diverges and indefinitely increases in time, which implies a clear nonstationary condition. This occurs because electrons with higher emission energy impact earlier and get quickly absorbed. Therefore, as time increases, the surviving electrons are those with lower emission energies and higher transit times, which implies an increasing multipactor order with time. The current stationary theory cannot model this situation, whereas the new non-stationary one matches the numerical simulations with extremely good agreement. Notice that the random dispersion on the numerical simulations for increasing time is due to the fact that a large amount of electrons have been already 
absorbed and therefore there are less samples available to produce smooth averages.

The multipactor boundaries calculated with stationary (reproducing the formulation of Ref. [VSS04]) and non-stationary theories together with numerical simulations are plotted in Fig. 4.24. The

\section{Figure 4.24.}

(Color online) Analytical and numerical Multipactor breakdown boundaries for silver. Experimental results are included.

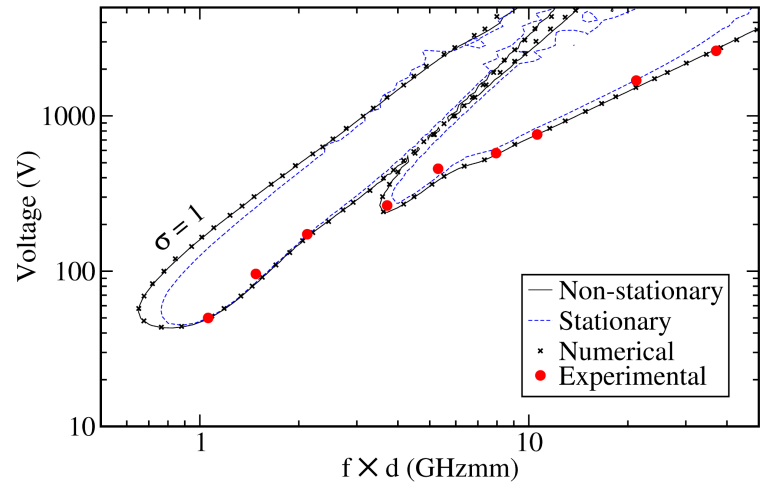

analytical boundaries are delimited by $\sigma_{a v}=1$, given by (4.44). The numerical ones are set by $\sigma_{e q}=1(4.46)$.

The non-stationary theory curve shows a better agreement with numerical simulations than the stationary one, concretely for the upper boundary regions and high $f \times d$ product, where the influence of single surface impacts is higher. The three curves are coincident for the regions close to the breakdown level. The experimental breakdown levels from report [WJ89] are also plotted, showing an excellent agreement with the theory. This evidences the capability of the statistical theories to predict the multipactor breakdown level in the whole $f \times d$ range, in contrast with the classical theories which are restricted to the first multipactor order.

SEY contour plots can be represented in Voltage versus $f \times d$ maps using (4.43). The isoline of $\sigma=1$ represent the multipactor breakdown boundary. Fig. 4.25 shows such representation for a $\sigma>1$ (top) and $\sigma<1$ (bottom). The isolines are plotted with a 0.2 and 0.1 step, respectively, starting from 1 .

In Fig. 4.25 (bottom), while the results for the region below the breakdown boundary perfectly agrees with numerical results, the regions above show a dissimilar trend. This is due probably to the 

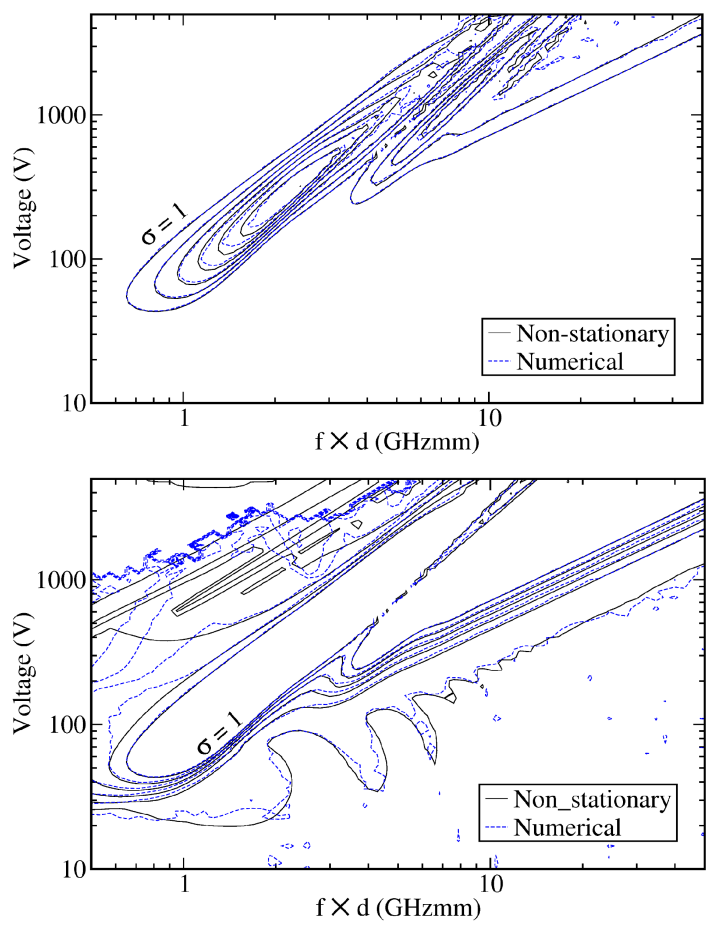

Figure 4.25.

(Color online) Analytical equivalent SEY calculated with (4.44)for silver and numerical simulations with FEST3D, for $\sigma>1$ (top) and $\sigma<1$ (bottom).

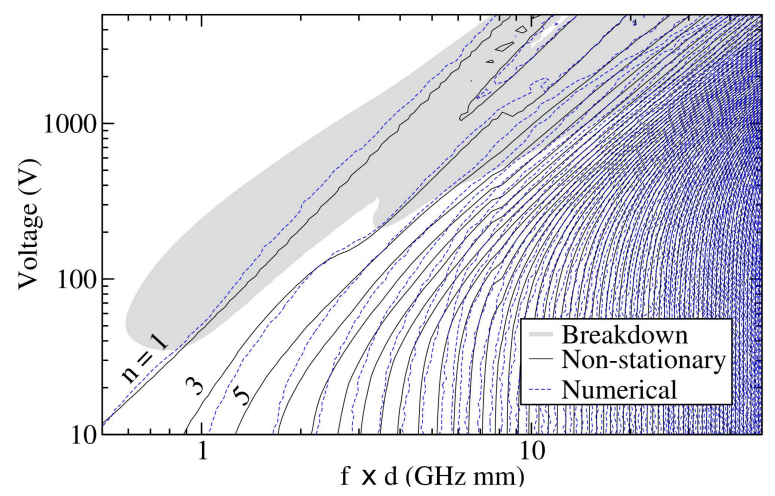

Figure 4.26.

(Color online) Analytical equivalent order calculated with (4.44) for custom material and numerical simulations with FEST3D. 
numerical noise of the simulations for very high fields, and the limited number of time samples in the electron trajectory computation.

These multipactor maps, coming from the non-stationary statistical theory, show a different structure of the SEY compared with the classical theory (see Fig 4.16). In contrast to the discontinuous band structure of the classical theory, the non-stationary theory shows a constant SEY value of 1 at the breakdown boundary which varies smoothly with the rf voltage. This is successfully contrasted with numerical simulations.

Finally, the contour plot for the equivalent order of the nonstationary theory (4.44) is shown in Fig. 4.26. The non-stationary theory is valid for any kind of material, provided that its SEY parameters are available. In this case, a custom material with SEY parameters $\sigma_{0}=0.5, \sigma_{m}=2.12, W_{1}=25 \mathrm{eV}$ and $W_{m}=364 \mathrm{eV}$ is used. The contour lines are plotted for odd integer values starting from 1 . In the multiplication region, the traditional band structure is observed, whereas for the region below breakdown the lines deflect to almost get vertically aligned with the plot. This is because for decreasing voltages, the transition time depends almost exclusively on the gap $d$ and the thermal spread $v_{t}$, and thus, the dependence on $f \times d$ is broken. The theory and numerical results match quite good in the multiplication region. Below that, the definition of a multipactor order is not valid anymore, because the process is then not stationary and the instantaneous multipactor order indefinitely increases in time.

\subsubsection{Conclusions}

A new non-stationary statistical multipactor theory has been presented. It is based on previous works on statistical multipactor but overcoming some of its limitations. Specifically, the stationarity assumption has been removed and single surface interactions have been incorporated to the model. In consequence, this new theory is able to accurately predict the time evolution of the most relevant parameters characterizing the multipactor process.

This theory provides realistic multipactor susceptibility charts including SEY and order contour plots for the whole $V$ versus $f \times d$ region, characterizing the dynamics of the discharge. These plots 
reveal a different SEY structure than the one predicted by the classical theory. Instead of a discontinuous sharp band structure, the SEY shows a smooth transition at the breakdown boundaries, where it has a value of 1 . This result is radically different from the one given by the classical theory and depicts a progressive and continuous change in the electron growth as the boundary is exceeded. This constitutes a much more realistic scenario of the electron dynamics.

The theory has been verified with numerical results coming from a PIC multipactor simulator and experimental results available in literature. The agreement between the theory, the numerical and the experimental results is excellent.

To the knowledge of the authors, this is the first theory which provides a framework for the study of a full polyphase electron interaction for both an exponential electron growth and a non-stationary electron absorption (when multipactor conditions are not met). This is of particular importance in non-stationary multipactor processes such as multicarrier operation and signal modulation. Therefore, this theory may constitute the basis for further study of multipactor in more complicated geometries and signals. 


\subsection{Multipactor theory for multi-carrier signals}

\subsubsection{Introduction}

Multipactor effect [Far34, GvE48, Vau88] is a high power RF breakdown occurring in vacuum or near vaccum microwave systems. In the multipactor discharge, electrons are accelerated by the electromagnetic fields, impacting against the device surfaces in synchronism with the rf signal frequency. Secondary electrons are released after each impact, due to secondary electron emission, developing an exponential charge growth which ultimately produces an electron discharge. Its effects range from signal degradation to the complete destruction of the component. Hence, the design of multipactor-free components is essential for the microwave industry, especially for RF space systems and particle accelerators.

Multipactor with multi-carrier signals is a key issue in space communications. Modern satellite payloads, for either defense or commercial communication applications (such as Digital Broadcast Satellite - DBS - Systems), tend to operate with an increasing number of communication channels and increasing power levels per carrier. This leads to extremely high peak power levels in the multi-carrier path of the spacecraft, which results in an ever increasing risk of multipactor discharge [SK01, GW96, RLR ${ }^{+}$94].

The classical Multipactor theory [GvE48, HW54, HW58, Vau88] covers single-carrier continuous wave $(\mathrm{CW})$ signals with parallel plates geometry (homogeneous electrostatic field). During the last 50 years, other works have extended it in order to cover more complex situations, such as single surface multipactor with magnetic transversal fields [RCD95], single surface multipactor with dielectrics $\left[\mathrm{KLA}^{+} 98\right]$, even and hybrid modes [Gil92, $\left.\mathrm{KBS}^{+} 02\right]$, other geometries, such as irises $\left[\mathrm{SRU}^{+} 08\right]$, rectangular waveguides $\left[\mathrm{SRA}^{+} 07\right]$ and coaxial structures [Woo68], as well as non-resonant or polyphase multipactor [VSS04, $\mathrm{KLS}^{+} 08$ ]. However, all of them remain restricted to the single-carrier case.

A typical multi-carrier signal is composed of several modulated carriers with small frequency separation. As a result, the multicarrier signal is a modulated rf signal with a time varying periodic 
envelope. Such a modulation adds a dynamical non-stationary behavior to the multipactor process which is not well understood yet.

There are few works dealing with multipactor in multi-carrier systems, and most of them are based, either on experimental results, or in Particle-in-Cell (PIC) codes and Montecarlo simulations. A specific multipactor theory for multi-carrier signals is not presented in any of them. For instance, the standard for multi-carrier multipactor design of European Space Agency (ESA) [EST03] is based on the "20gap-crossing rule", which states that multipactor takes place only if the multi-carrier signal envelope exceeds the breakdown voltage for a time equal or higher than the time that an electron takes to cross the gap 20 times $\left(T_{20}\right)$. This rule establishes a multipactor criterion for a single envelope peak. However, in Ref. [AVG $\left.{ }^{+} 07\right]$, a new mechanism of long-term multipactor in multi-carrier applications is addressed, in which, contrarily to the "20-gap-crossing rule", the charge accumulates between consecutive periods of the envelope. In Ref. [SVB $\left.{ }^{+} 03\right]$, numerical results of electron population growth for multi-carrier signals and infinite parallel plates are presented. Finally, a numerical study of multipactor with a two carrier signal is given by Semenov et al. in Ref. [SK01].

When seeking for a multipactor theory with multi-carrier signals, the main obstacle to overcome is its inherent non-stationarity. The non-stationary theory for single-carrier signals published in Ref. $\left[\mathrm{AVG}^{+} 10\right]$ belongs to the family of statistical multipactor theories [VSS04]. It performs an statistical analysis of the multipactor discharge working with probabilities rather than deterministic values (as the classical theories do). The key feature of the non-stationary theory is that it separately characterizes the dynamics of single surface and double surface interactions for each of the two metal plates. On the other hand, it is not restricted to the study of the probabilities over a period of the rf signal, but rather extends the time window to infinity. As a consequence, the theory is able to model the complete electron dynamics for stationary and non-stationary situations. Although the theory was initially formulated with a monochromatic time-harmonic function as the driving electric field, by definition it should not be limited to such simple trigonometric function. In fact, we will show that the non-stationary theory accepts arbitrary functions for the driving electric field signal, as long as they are periodic. 
Consequently, this theory is the best candidate for a general multipactor theory for multi-carrier signals.

In this work we present a generalization of the non-stationary statistical theory for multipactor with single-carrier signals presented in Ref. $\left[\mathrm{AVG}^{+} 10\right]$. We will show how, by redefining the equations of motion, in order to incorporate an arbitrary number of carriers, and some key changes in the basic formulation, the non-stationary theory can be generalized to deal with multi-carrier signals without loosing or degrading any of its previous potential. This theory, following the original work of Ref. $\left[\mathrm{AVG}^{+} 10\right]$, assumes an infinite parallel-plate geometry, since it implies a uniform field distribution and, therefore, admits a one-dimensional solution for the equations of motion. Most of the publications in multipactor theory are based on parallel-plate geometries (see for example Refs. [GvE48, HW54, HW58, RCD95, KLA ${ }^{+}$98, Gil92, $\mathrm{KBS}^{+} 02$, Vau88, $\mathrm{AVG}^{+} 10, \mathrm{SVB}^{+} 03$, SK01, VSS04, $\mathrm{KLS}^{+} 08$ ]) and, in spite of its simplicity, it is generally accepted as a valid framework for understanding the physics of the process. Extensions of this theory to other geometries and more particular field distributions may be subject of further studies.

First, the multi-carrier signals and its properties are summarized in Section 4.4.2. Then, the new theory for multi-carrier signals is presented in Section 4.4.3 and finally verified with numerical results in Sections 4.4.4 and 4.4.5.

\subsubsection{Multi-carrier signal analysis}

A multi-carrier signal $E(t)$, composed of $K$ carriers with amplitudes $E_{i} \in \mathrm{E}$, angular frequencies $\omega_{i} \in \Omega$, and phases $\phi_{i} \in \Phi, i=1, \ldots, K$, has the form

$$
E(t)=\sum_{i=1}^{K} E_{i} \cos \left(\omega_{i} t+\phi_{i}\right) .
$$

Without any loss of generality we assume that $\omega_{1}<\omega_{2}<\ldots<\omega_{K}$. The sets of amplitudes $E_{i} \in \mathrm{E}$, angular frequencies $\omega_{i} \in \Omega$ and phases $\phi_{i} \in \Phi$, completely characterize a specific multi-carrier signal.

Let $E^{+}(t)$ be the analytic signal of $E(t)$, defined as $E^{+}(t)=$ $1 / \pi \int_{0}^{\infty} U(\omega) \exp (j \omega t) d \omega$, where $U(\omega)$ is the Fourier transform of $E(t)$, defined as $U(\omega)=\int_{-\infty}^{\infty} E(t) \exp (-j \omega t) d t$. From (4.48), $E^{+}(t)$ 
is simply

$$
E^{+}(t)=\sum_{i=1}^{K} E_{i} \exp \left[j\left(\omega_{i} t+\phi_{i}\right)\right]
$$

An alternative representation of the analytic signal, often used for narrow-band signals, is done by using the envelope, $A(t)$, and instantaneous phase, $P(t)$,

$$
E^{+}(t)=A(t) \exp (j P(t))
$$

where

$$
A(t)=\left|E^{+}(t)\right|=\sqrt{R^{2}(t)+I^{2}(t)},
$$

and

$$
P(t)=\arg \left(E^{+}(t)\right)=\left\{\begin{array}{ll}
\tan ^{-1}\left(\frac{I(t)}{R(t)}\right), & R(t) \geq 0 \\
\tan ^{-1}\left(\frac{I(t)}{R(t)}\right)+\pi, & R(t)<0
\end{array},\right.
$$

These expressions use the real and imaginary parts of the analytic signal

$$
R(t)=\sum_{i=1}^{K} E_{i} \cos \left(\omega_{i} t+\phi_{i}\right)
$$

and

$$
I(t)=\sum_{i=1}^{K} E_{i} \sin \left(\omega_{i} t+\phi_{i}\right),
$$

respectively. Note that (4.52) is undefined for $R(t)=I(t)=0$, which also implies $A(t)=0$.

In turn, the instantaneous frequency is defined as

$$
F(t)=\frac{1}{2 \pi} \frac{d P(t)}{d t}=\frac{1}{2 \pi} \frac{I^{\prime}(t) R(t)-I(t) R^{\prime}(t)}{R^{2}(t)+I^{2}(t)},
$$

$R^{\prime}(t)$ and $I^{\prime}(t)$ being the time derivatives of the real and imaginary parts of the analytic signal, respectively. In the same way as (4.52), the instantaneous frequency is undefined when $R(t)=I(t)=0$.

The period $T$ of the multi-carrier signal is found by solving the following relationship

$$
E^{+}(t+T)=E^{+}(t)=\sum_{i=1}^{K} E_{i} \exp \left[j\left(\omega_{i} t+\phi_{i}\right) \exp \left(\omega_{i} T\right)\right]
$$


which leads to the Diophantine equations

$$
T=n_{1} T_{1}=n_{2} T_{2}=\ldots=n_{K} T_{K} \quad n_{i} \in \mathbb{N},
$$

with $T_{i}=2 \pi / \omega_{i}=1 / f_{i}$, where $2 \pi f_{i}=\omega_{i}$. The solution exists only if $f_{i} \in \mathbb{Q}, \quad \forall i$. Otherwise, the multi-carrier signal is non-periodic. If $f_{i}$ are integers, the solution is obtained by finding the greatest common divisor, $\operatorname{gcd}()$ of the set of frequencies

$$
T=1 / f, \quad f=\operatorname{gcd}\left(f_{1}, f_{2}, \ldots f_{n}\right)
$$

In practical situations, the precision of the carrier frequencies is limited to a certain number of decimals. Therefore, it is always possible to normalize such quantities to obtain integer frequencies and solve (4.58).

The above condition is very restrictive and forces an absolute periodicity of both envelope and phase of the signal. However, a relative periodicity only for the amplitude may be forced, i.e. after a period $\tilde{T} \leq T$ the signal has the same amplitude but different phase $\alpha \in[0,2 \pi]$,

$$
\begin{aligned}
E^{+}(t+\tilde{T})= & E^{+}(t) \exp (j \alpha)= \\
& \sum_{i=1}^{K} E_{i} \exp \left[j\left(\omega_{i} t+\phi_{i}\right)\right] \exp \left(j \omega_{i} \tilde{T}\right),
\end{aligned}
$$

or equivalently

$$
\begin{aligned}
& A(t+\tilde{T})=A(t), \\
& P(t+\tilde{T})=P(t)+\alpha, \\
& F(t+\tilde{T})=F(t) .
\end{aligned}
$$

This reveals that the relative period $\tilde{T}$ is actually the period for the envelope and instantaneous frequency, which may be shorter than the absolute one. This period can be solved by factoring out one of 
the frequencies from (4.59), for instance the first one:

$$
\begin{aligned}
& E^{+}(t+\tilde{T})=E^{+}(t) \exp (j \alpha)= \\
& \exp \left[j\left(\omega_{1} \tilde{T}\right)\right]\left\{E_{1} \exp \left[j\left(\omega_{1} t+\phi_{1}\right)\right]+\right. \\
& \left.+\sum_{i=2}^{K} E_{i} \exp \left[j\left(\omega_{i} t+\phi_{i}\right)\right] \exp \left[j\left(\omega_{i}-\omega_{1}\right) \tilde{T}\right]\right\} .
\end{aligned}
$$

This leads again to the Diophantine equations

$$
\tilde{T}=\tilde{n_{2}} \tilde{T}_{2}=\ldots=\tilde{n_{K}} \tilde{T_{K}} \quad \tilde{n_{i}} \in \mathbb{N},
$$

with $\tilde{T}_{i}=1 /\left(f_{i}-f_{1}\right), \quad i=2, \ldots, K$.

Analogously to (4.58), the solution needs to find the greatest common divisor of the differences between the signal frequencies and the lowest one.

$$
\tilde{T}=1 / \tilde{f} \quad \tilde{f}=\operatorname{gcd}\left(f_{2}-f_{1}, f_{3}-f_{1}, \ldots, f_{n}-f_{1}\right)
$$

Thanks to the inherent properties of the gcd function, it can be demonstrated that the solution is the same independently on the frequency chosen for factoring out. It is obvious from (4.56) and (4.59)(4.61) that the absolute period must be a multiple of the relative period, $T=k \tilde{T}, \quad k \in \mathbb{N}$ and that the phase is $\alpha=\omega_{1} \tilde{T}=\omega_{1} T / k$.

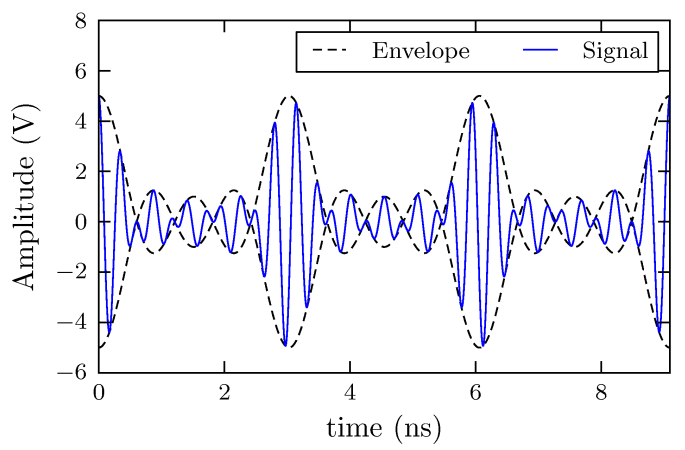

Figure 4.27.

(Color online) Uniformly frequency spaced, 5 carrier signal with parameters of Table 4.7 and zero phase condition.

Figures 4.27 and 4.28 show the signal, envelope and instantaneous frequency of a uniformly spaced 5 carrier signal, with two different 
Figure 4.28.

(Color online)Uniformly frequency spaced, 5 carrier signal with parameters of Table 4.7. a) Envelope, b) Instantaneous frequency
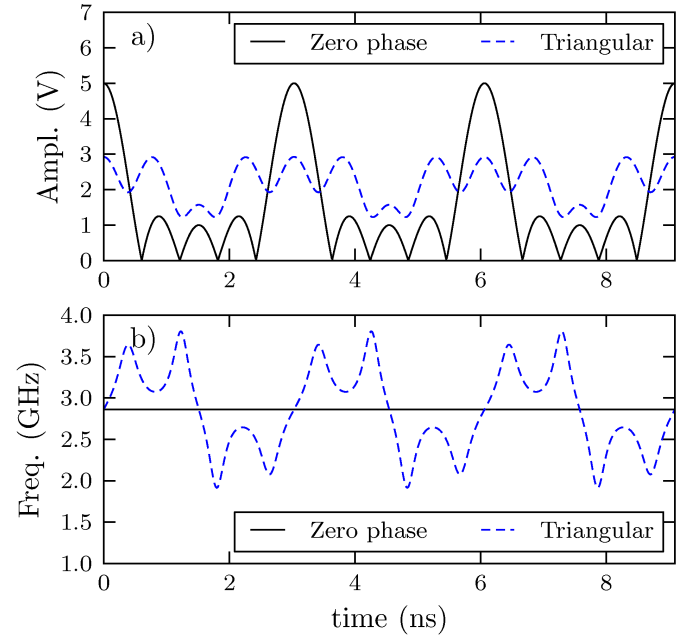

phase conditions. The phase 1 is the zero phase condition (all phases are set to 0 ), whereas the phase 2 is a triangular phase condition. All carriers are $330 \mathrm{MHz}$ spaced. The amplitudes are set to $1 \mathrm{~V} / \mathrm{m}$ for all carriers. Table 4.7 details the frequency and phase for all carriers.

Table 4.7. 5 carrier signal parameters. Amplitude is $1 \mathrm{~V} / \mathrm{m}$ for all carriers

\begin{tabular}{cccc} 
carrier & frequency $(\mathrm{GHz})$ & phase $1(\mathrm{deg})$ & phase $2(\mathrm{deg})$ \\
\hline 1 & 2.20 & 0 & 0 \\
2 & 2.53 & 0 & 75.35 \\
3 & 2.86 & 0 & 150.7 \\
4 & 3.19 & 0 & 75.35 \\
5 & 3.52 & 0 & 0 \\
\hline
\end{tabular}

The absolute and relative periods of this signal (which is independent of the phase scheme) can be computed with (4.58) and (4.63), respectively. The frequencies are such that $T=9.09 \mathrm{~ns}$, being the relationship between the periods $T=3 \tilde{T}$. This can be appreciated in Fig 4.27, in which one period of the multi-carrier signal is plotted. 
The period of the signal (4.48) contains three periods of the envelope (4.51).

In Fig. 4.28 the effect of the different phase schemes can be noticed in both the envelope (a) and the instantaneous frequency (b). Although both have the same period, the zero phase signal presents a higher and narrower main lobe of the envelope, whilst the triangular phase signal shows a flatter behavior. With respect to the instantaneous frequency, the zero phase scheme, with the uniform frequency spacing and equal amplitude conditions, has a constant instantaneous frequency equal to the average frequency, $f_{m}=2.86 \mathrm{GHz}$. On the contrary, for the triangular case, the instantaneous frequency oscillates around that value with considerable peaks above and below such value.

Fig. 4.29 shows the envelope and instantaneous frequency of another example of multi-carrier signal, i.e. a 10 carrier signal with non-uniform frequency spacing and arbitrary amplitudes and phases. Table 4.8 shows the parameters of the signal. The spacing is of 80 $\mathrm{MHz}$ for the first five carriers and $120 \mathrm{MHz}$ for the others. This combination of frequencies makes that $T=40 \tilde{T}$, being $T=1 \mu \mathrm{s}$. This means that a period of the signal contains 40 periods of the envelope. In Fig. 4.29, only a relative period is plotted since it is enough for representing the envelope and the instantaneous frequency. As it happens in the previous example, the instantaneous frequency oscillates around the average frequency, $f_{m}=5.947 \mathrm{GHz}$.

Table 4.8. 10 carrier signal parameters.

\begin{tabular}{cccc} 
carrier & frequency $(\mathrm{GHz})$ & amplitude $(\mathrm{V} / \mathrm{m})$ & phase $(\mathrm{deg})$ \\
\hline 1 & 5.527 & 1 & 0 \\
2 & 5.607 & 1.25 & 15 \\
3 & 5.687 & 0.8 & 45 \\
4 & 5.767 & 0.5 & 123 \\
5 & 5.847 & 2 & 7.5 \\
6 & 5.967 & 1 & 0 \\
7 & 6.087 & 1.1 & 240 \\
8 & 6.207 & 0.95 & 180 \\
9 & 6.327 & 0.95 & 315 \\
10 & 6.447 & 1.12 & 240 \\
\hline
\end{tabular}


Figure 4.29.

Arbitrary 10 carrier signal with parameters of Table 4.8. a) Envelope, b) Instantaneous frequency.
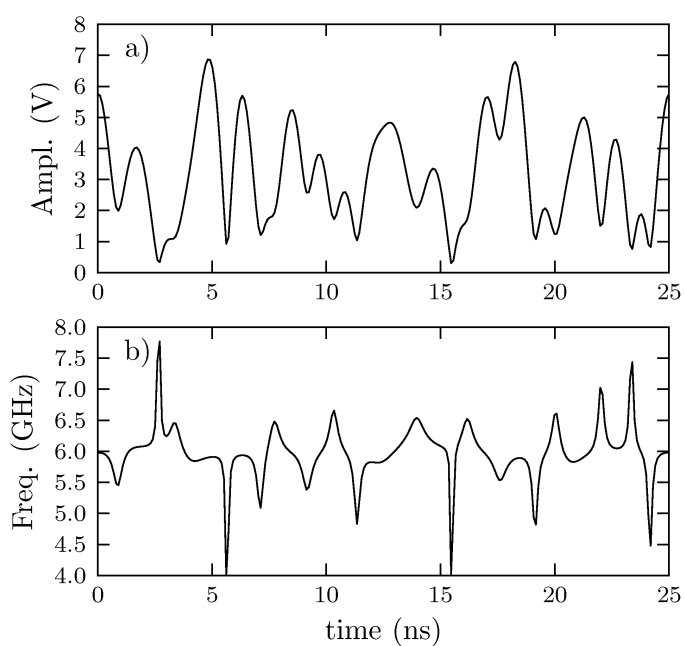

This analytical study of the multi-carrier signal allows to obtain two interesting conclusions. First, in general, the period of the signal and the period of the envelope are different (they may be equal for certain frequency combinations). More specifically, the period of the signal is an integer multiple of the envelope period, which has to be considered in any analytical development employing a multicarrier signal. Second, in general, the instantaneous frequency is not constant with time and may differ considerably from the average frequency. This fact limits the validity of previous multipactor approaches for multi-carrier signals, such as the 20-gap-crossing rule [EST03], which apply the multipactor single carrier theory considering a fixed frequency equal to the lowest frequency among all carriers.

\subsubsection{Multipactor theory for multi-carrier signals}

\subsubsection{Equations of motion}

The formulation of the non-stationary theory starts from the onedimensional equations of motion of an electron between two infinite parallel plates located at $x=0$ and $x=d$. The electron mass and 
charge are $m_{e}$ and $-e$ respectively. The electron is subject to of a uniform electric field in the $x$ direction, $E(t)$. The force acting on the electron follows the Lorentz law $f(t)=-e E(t)$.

The main innovation with respect to the existing multipactor theories is that, here, the driving electric field is an arbitrary multicarrier signal of the form given in Section 4.4.2, instead of the classical monochromatic time-harmonic function. The acceleration exerted on the electron is then

$$
m_{e} \ddot{x}=e \sum_{i=1}^{K} E_{i} \sin \left(\omega_{i} t+\phi_{i}\right) .
$$

The voltage between plates is simply $V_{i}=E_{i} d$ and $V(t)=E(t) d$.

In (4.64) the multi-carrier signal is taken as

$$
E(t)=\sum_{i=1}^{K}-E_{i} \sin \left(\omega_{i} t+\phi_{i}\right)
$$

which is equivalent to (4.48) and only supposes an absolute temporal shift of $-\pi / 2$ which does not imply any loss of generality. This has been done to preserve the existing formulation of the non-stationary theory $\left[\mathrm{AVG}^{+} 10\right]$.

The electron velocity and position can be derived by simple integration of (4.64). For an electron released from $x=0$ at time $t_{s}$ with initial velocity $v_{0}$, we have

$$
\dot{x}=v_{0}+\frac{e}{m_{e}} \sum_{i=1}^{K} \frac{E_{i}}{\omega_{i}}\left[\cos \left(\omega_{i} t_{s}+\phi_{i}\right)-\cos \left(\omega_{i} t+\phi_{i}\right)\right],
$$

and

$$
\begin{aligned}
x & =v_{0}\left(t-t_{s}\right)+\frac{e}{m_{e}} \sum_{i=1}^{K}\left\{\frac{E_{i}}{\omega_{i}} \cos \left(\omega_{i} t_{s}+\phi_{i}\right)\left(t-t_{s}\right)+\right. \\
& \left.+\frac{E_{i}}{\omega_{i}^{2}}\left[\sin \left(\omega_{i} t_{s}+\phi_{i}\right)-\sin \left(\omega_{i} t+\phi_{i}\right)\right]\right\},
\end{aligned}
$$

for the velocity and position, respectively.

The equations must be normalized in order to work with more useful expressions with adimensional quantities. The frequency of 
the multi-carrier period, $\omega=2 \pi / T$ [see (4.56)-(4.58)] , and the amplitude of the first carrier, $E_{1}$, have been arbitrarily chosen for normalization, although the following development could be reproduced for any other with the same results. Thus, both sides of (4.66) are multiplied by $\omega / v_{\omega}$, where $v_{\omega}=e E_{1} /\left(m_{e} \omega\right)$. The following notation has been employed

$$
\begin{array}{r}
\xi=\omega x / v_{\omega}, \quad \varphi=\omega t, \quad \varphi_{s}=\omega t_{s}, \\
u=v_{0} / v_{\omega}, \quad \lambda=\omega d / v_{\omega} \\
\Delta E_{i}=E_{i} / E_{1} \quad \Delta \omega_{i}=\omega_{i} / \omega,
\end{array}
$$

Note that $\lambda$ represent the normalized distance between plates. This yields the following equation for the normalized position

$$
\begin{aligned}
& \xi=u \tau+\sum_{i=1}^{K}\left\{\frac{\Delta E_{i}}{\Delta \omega_{i}} \cos \left(\Delta \omega_{i} \varphi_{s}+\phi_{i}\right) \tau+\right. \\
& \left.+\frac{\Delta E_{i}}{\Delta \omega_{i}^{2}}\left[\sin \left(\Delta \omega_{i} \varphi_{s}+\phi_{i}\right)-\sin \left(\Delta \omega_{i}\left(\tau+\varphi_{s}\right)+\phi_{i}\right)\right]\right\},
\end{aligned}
$$

and the normalized velocity $\dot{\xi}=d \xi / d \tau$

$$
\begin{aligned}
\dot{\xi}= & u+\sum_{i=1}^{K} \frac{\Delta E_{i}}{\Delta \omega_{i}}\left[\cos \left(\Delta \omega_{i} \varphi_{s}+\phi_{i}\right)-\right. \\
& \left.-\cos \left(\Delta \omega_{i}\left(\tau+\varphi_{s}\right)+\phi_{i}\right)\right]
\end{aligned}
$$

where $\tau=\varphi-\varphi_{s}$ is the transit phase.

With this normalization, it is remarkable that the range $\varphi_{s} \in$ $[0,2 \pi]$ covers one period of the multi-carrier signal, since for $t_{s}=$ $T \rightarrow \varphi_{s}=2 \pi$. Hence, the electron trajectory given by (4.68) is periodic with respect to $\varphi_{s}$, i.e.

$$
\xi\left(\varphi_{s}+2 \pi\right)=\xi\left(\varphi_{s}\right)
$$

\subsubsection{Multipactor theory for multi-carrier signals}

The original notation of Ref. $\left[\mathrm{AVG}^{+} 10\right]$ is kept. Each plate is denoted as " $D$ " and " $U$ " for the boundary conditions $\xi=0$ (Down) and $\xi=\lambda$ (Up), respectively. Thus two kinds of interactions are considered: 
double surface $(D-U$ or $U-D$ trajectories) and single surface $(D-D$ and $U-U$ trajectories). All their related parameters are properly labeled as " $d s$ " and "ss", respectively. $\varphi=\omega t$ represents the absolute phase. Fig. 4.30 depicts these concepts.

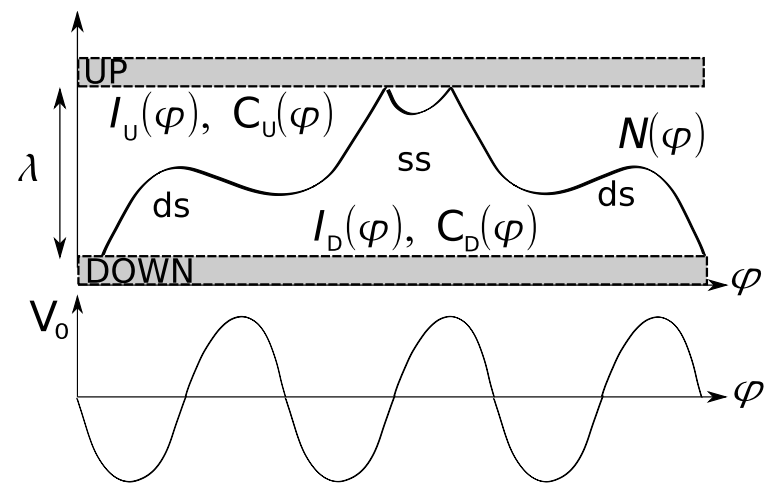

Figure 4.30.

Non-stationary definitions and conventions. Some possible electron trajectories are depicted and labeled with the corresponding type of interaction, double surface (ds) and single surface (ss).

The statistical multipactor theories $\left[\mathrm{VSS} 04, \mathrm{AVG}^{+} 10\right]$ are constructed upon the Transit Time Probability Density (TTPD) function, namely $G$. Originally, $G\left(\tau \mid \varphi_{s} ; \lambda\right)$, is defined as the probability density function of the transit phase $\tau$ for an electron traveling from 0 to the opposite plate, $\xi=\lambda$, being released at phase $\varphi_{s} \in[0,2 \pi]$.

In this work, we propose a generalization of the TTPD to consider arbitrary positions $\chi \in[0, \lambda]$ and to include the parameters $\mathrm{E}, \Omega, \Phi$, in order to handle arbitrary multi-carrier signals. Therefore, $G\left(\tau \mid \varphi_{s} ; \chi, \mathrm{E}, \Omega, \Phi\right)$, is defined as the probability density function of the travel phase $\tau=\varphi-\varphi_{s}$ for an electron traveling from $\xi=0$ to $\xi=\chi$, being released at phase $\varphi_{s}$ and for a multi-carrier signal defined by the sets $\mathrm{E}, \Omega$, and $\Phi$. If $\chi=\lambda$, the TTPD is particularized for a transit between opposite plates and thus a double surface interaction is considered. On the contrary, if $\chi=0$, the TTPD represents all the interactions coming back to the emission surface, i. e. single surface impacts.

In order to construct $G\left(\tau \mid \varphi_{s} ; \chi, \mathrm{E}, \Omega, \Phi\right)$ the theorem of transformation of Univariate Random Variables is employed. It starts from the known relationships between $u$ and $\tau$, given by the equations of motion (4.68), (4.69), and the probability density function of the electron emission velocity $u$, namely $f_{u}(u)$, which is known a-priori. 
A variety of models are available in the literature $\left[\operatorname{Ver} 05, \mathrm{AVR}^{+} 08\right.$, FP02, CE74]. According to this theorem, $G\left(\tau \mid \varphi_{s} ; \chi, \mathrm{E}, \Omega, \Phi\right)$ can be written as

$$
G\left(\tau \mid \varphi_{s} ; \chi\right)=\left|\frac{d g\left(\tau \mid \varphi_{s} ; \chi\right)}{d \tau}\right| f_{u}\left(g\left(\tau \mid \varphi_{s} ; \chi\right)\right),
$$

where $u=g\left(\tau \mid \varphi_{s} ; \chi\right)$ must be a monotonic function which expresses $u$ as a function of $\tau$. From now on we will consider a general multicarrier case, and therefore the terms E, $\Omega, \Phi$ are omitted for the sake of brevity, except when needed.

By setting $\xi=\chi$ in (4.68), $u$ can be easily obtained, yielding the candidate function $g_{0}\left(\tau \mid \varphi_{s} ; \chi\right)$

$$
\begin{aligned}
& u=g_{0}\left(\tau \mid \varphi_{s} ; \chi\right)= \\
& \frac{\chi+\sum_{i=1}^{K} \frac{\Delta E_{i}}{\Delta \omega_{i}^{2}}\left\{\sin \left[\Delta \omega_{i}\left(\tau+\varphi_{s}\right)+\phi_{i}\right]-\sin \left(\Delta \omega_{i} \varphi_{s}+\phi_{i}\right)\right\}}{\tau} \\
& -\sum_{i=1}^{K} \frac{\Delta E_{i}}{\Delta \omega_{i}} \cos \left(\Delta \omega_{i} \varphi_{s}+\phi_{i}\right) .
\end{aligned}
$$

The candidate $g_{0}$ is, in general, a non-monotonic function. Therefore, such a non-monotonicity must be removed to obtain $g$ and solve (4.70). This forces $G\left(\tau \mid \varphi_{s} ; \chi\right)$ to be 0 in the non-monotonic intervals of $g_{0}$.

From the equations of motion of Section 4.4.3.1 and the above definitions, it is obvious that the single carrier non-stationary theory of Ref. $\left[\mathrm{AVG}^{+} 10\right]$ is a particular case with $K=1$ of the multi-carrier theory presented in this work.

The above equations assume a starting position 0 and are valid for an arbitrary final position $\chi \in[0, \lambda]$, but two specific solutions are of particular interest: $\chi=\lambda$, corresponding to double surface interactions and $\chi=0$, for single surface interactions. Therefore, the double surface and single surface emission velocities are defined as $u_{d s} \equiv g\left(\tau \mid \varphi_{s} ; \lambda\right)$ and $u_{s s} \equiv g\left(\tau \mid \varphi_{s} ; 0\right)$, respectively. The minimum ejection velocity for double surface is equal to the maximum ejection 
velocity for single surface, i.e. $\min \left(u_{d s}\right)=\max \left(u_{s s}\right)=u_{t h} \geq 0$. When $\varphi_{s}$ is such that $u_{t h}=0$, no single surface interactions can occur for that specific emission phase.

Fig. 4.31 shows a plot of $u_{d s}$ and $u_{s s}$ for a 6 carrier signal with uniform frequency spacing of $100 \mathrm{MHz}$, equal amplitude $V_{0}=30 \mathrm{~V}$ and zero phase. Table 4.9 shows the frequencies for all carriers. The gap and starting phase are $d=0.43 \mathrm{~mm}$ and $\varphi_{s}=0.27^{\circ}$, respectively. The inner plot shows that both functions present local oscillations which are related to the periodic variations of the envelope.

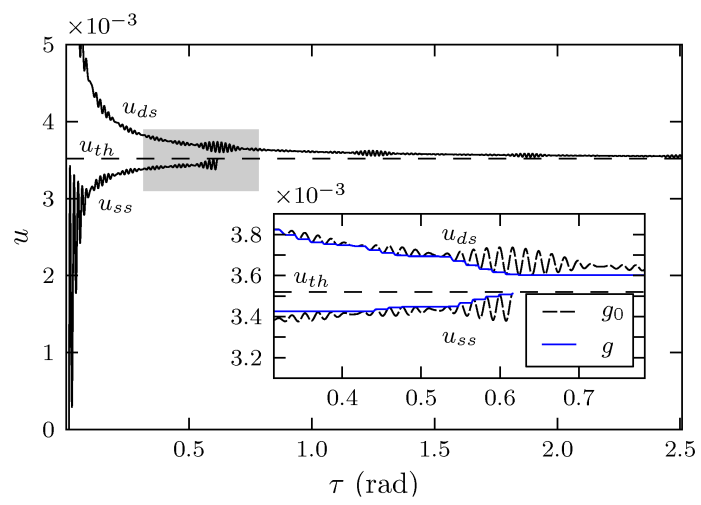

\begin{tabular}{cc} 
carrier & frequency $(\mathrm{GHz})$ \\
\hline 1 & 3.57 \\
2 & 3.67 \\
3 & 3.77 \\
4 & 3.87 \\
5 & 3.97 \\
6 & 4.07 \\
\hline
\end{tabular}

\section{Figure 4.31.}

(Color online) Example of $u_{d s}$ and $u_{s s}$ for the 6 carrier signal of Table 4.9, equal amplitude $V_{0}=30 \mathrm{~V}$, zero phase, $d=0.43 \mathrm{~mm}$ and $\varphi_{s}=$ $0.27^{\circ}$. The inner window represents the enlargement of the shaded area.

\section{Table 4.9.}

6 carrier signal parameters.

Table 4.10 introduces the most relevant definitions of the nonstationary theory, which constitute the basis of its development. Next, we will derive the analytical expressions for such definitions.

Firstly, we need to particularize $G\left(\tau \mid \varphi_{s} ; \chi\right)$ for each kind of interaction and starting plate to obtain the TTPD functions defined in Table 4.10. As it was stated above, $G\left(\tau \mid \varphi_{s} ; \chi\right)$ is defined only for the 
Table 4.10. Non-stationary theory definitions.

\begin{tabular}{lr}
\hline Impact rate (electrons / radian) in plate $U / D$ & $I_{U / D}(\varphi)$ \\
at phase $\varphi$ & $C_{U / D}(\varphi)$ \\
\hline Emission rate (electrons / radian) in plate & $N(\varphi)$ \\
$U / D$ at phase $\varphi$ & \\
\hline Number of electrons at phase $\varphi$ & \\
\hline TTPD of an electron starting at plate $U / D$, & \\
with phase $\varphi$, which experiences a dou- & $G_{d s / s s, U / D}(\tau \mid \varphi)$ \\
ble/single surface impact in a transit phase & \\
$\tau$ & \\
\hline SEY of an electron starting at plate $U / D$, & \\
with starting phase $\varphi$ which experiences a & $\sigma_{d s / s s, U / D}(\tau \mid \varphi)$ \\
double/single surface impact in a transit & \\
phase $\tau$ &
\end{tabular}

transitions starting from the plate $D(\xi=0)$ and for $\varphi_{s} \in[0,2 \pi]$. However, since the electron trajectory is periodic with $\varphi_{s}$ having a period of $2 \pi$ [see (4.69)], the function $G\left(\tau \mid \varphi_{s} ; \chi\right)$ is also periodic, $G\left(\tau \mid \varphi_{s}+2 \pi ; \chi\right)=G\left(\tau \mid \varphi_{s} ; \chi\right)$. This allows us to express the function in terms of the absolute phase $\varphi, G(\tau \mid \varphi ; \chi)=G\left(\tau \mid \varphi_{s} ; \chi\right), \quad \varphi_{s}=$ $\bmod (\varphi ; 2 \pi)$. On the other hand, an electron starting from the plate $U$ at phase $\varphi$, will follow the same trajectory (in opposite direction) as if it started from plate $D$ but with reversed field. Therefore, the TTPD of an electron starting from the plate $U$ is $G_{U}\left(\tau \mid \varphi_{s} ; \chi\right)=$ $G\left(\tau \mid \varphi_{s} ; \chi,-\mathrm{E}, \Omega, \Phi\right)$.

Summarizing, the TTPD functions of Table 4.10 are

$$
\begin{aligned}
& G_{d s, D}(\tau \mid \varphi)=G\left(\tau \mid \varphi_{s} ; \chi, \mathrm{E}, \Omega, \Phi\right) \mid \begin{array}{l}
\chi=\lambda \\
\varphi_{s}=\bmod (\varphi ; 2 \pi)
\end{array} \\
& G_{d s, U}(\tau \mid \varphi)=G\left(\tau \mid \varphi_{s} ; \chi,-\mathrm{E}, \Omega, \Phi\right) \mid \begin{array}{l}
\chi=\lambda \\
\varphi_{s}=\bmod (\varphi ; 2 \pi)
\end{array} \\
& G_{s s, D}(\tau \mid \varphi)=G\left(\tau \mid \varphi_{s} ; \chi, \mathrm{E}, \Omega, \Phi\right) \mid \begin{array}{l}
\chi=0 \\
\varphi_{s}=\bmod (\varphi ; 2 \pi)
\end{array} \\
& G_{s s, U}(\tau \mid \varphi)=G\left(\tau \mid \varphi_{s} ; \chi,-\mathrm{E}, \Omega, \Phi\right) \\
& \begin{array}{l}
\chi=0 \\
\varphi_{s}=\bmod (\varphi ; 2 \pi)
\end{array}
\end{aligned}
$$


where the subscripts $d s / s s, U / D$ indicate the particularization for an specific interaction and plate, and $\varphi \in[0, \infty]$ is the absolute phase. Note that although the parameters $\mathrm{E}, \Omega, \Phi$ have been omitted for brevity, the above definitions of the TTPD functions are valid for a general multi-carrier case.

The functions are normalized (independently for $D$ and $U$ plates) such that

$$
\int_{0}^{\infty} G_{d s}(\tau \mid \varphi) d \tau=P_{d s}(\varphi) \text { and } \int_{0}^{\infty} G_{s s}(\tau \mid \varphi) d \tau=P_{s s}(\varphi),
$$

where $P_{d s}(\varphi)$ and $P_{s s}(\varphi)$ are the probability that an electron emitted at $\varphi$ suffers a double or single surface impact, respectively. Note that $P_{d s}(\varphi)+P_{s s}(\varphi)=1$.

It is remarkable that, although the theory presented in this work is particularized for multi-carrier signals, it can be deduced from the above reasoning that the formulation is still valid for any signal which satisfies (4.69), for which the only condition of periodicity is required.

These definitions are valid for any probability distribution function of the electron initial velocity, $f_{u}(u)$. There are different models of such secondary electron emission energy in the literature. In this case, a typical Maxwellian distribution for the initial velocity is taken, since it is simple and fits reasonably well with experimental measurements [VSS04,DS05]. The Maxwellian distribution is given by

$$
f_{u}=\frac{u v_{\omega}^{2}}{v_{t}^{2}} \exp \left(-\frac{u^{2} v_{\omega}^{2}}{2 v_{t}^{2}}\right)
$$

where $v_{t}$ is the velocity thermal spread. A value of $W_{t}=1.5 \mathrm{eV}$ has been taken for this work, where $W_{t}=\frac{m_{e}}{2 e} v_{t}^{2}$ is the thermal emission energy spread.

The $G_{d s}$ and $G_{s s}$ functions corresponding to the example of Fig. 4.31 are presented in Fig. 4.32. The possible electron transit times are restricted to the regions where the $G_{d s}$ and $G_{s s}$ are not zero.

This specific example shows that the $G_{s s}$ is delimited to a region approximately between $\tau \in[1.1,1.6] \cdot 10^{-2}$, which means that no single-surface impacts can take place outside this interval. On the other hand, the $G_{d s}$ function is also limited to certain intervals but 
Figure 4.32.

(Color online) Example of $G_{d s}$ and $G_{s s}$ for the 6 carrier signal of Table 4.9, equal amplitude $V_{0}=30 \mathrm{~V}$, zero phase, $d=0.43 \mathrm{~mm}, \varphi_{s}=0.27^{\circ}$ and the Maxwellian distribution of (4.73) with $W_{t}=1.5 \mathrm{eV}$. The inner window represents the enlargement of the shaded area.

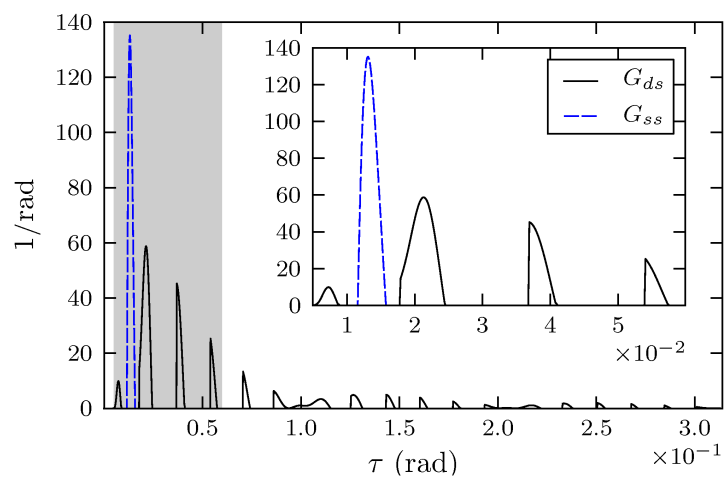

which spread to higher values of $\tau$. A value of $\tau=2 \pi$ represents one period of the multi-carrier signal, and both functions are practically 0 for a $\tau>0.3$. This implies that the impact will occur most probably at the beginning of the period (starting from $\varphi_{s}$ ). There is no overlap between both functions, but it may happen for certain combinations of starting phase, amplitudes, phases and frequencies of the multicarrier signal, $\varphi, \mathrm{E}, \Omega, \Phi$, respectively. An overlap of both functions at a certain interval implies that the electrons impacting at such transit time could have either experienced a single surface impact or double surface impact with different probability $\left[\mathrm{AVG}^{+} 10\right]$.

Once the TTPD functions are obtained, the rest of the definitions given in Table 4.10 can be derived in a similar way to the original non-stationary theory $\left[\mathrm{AVG}^{+} 10\right]$. Therefore, we have

$$
\begin{aligned}
& C_{U}(\varphi)= \\
& =\int_{0}^{\varphi} C_{D}\left(\varphi^{\prime}\right) G_{d s, D}\left(\varphi-\varphi^{\prime} \mid \varphi^{\prime}\right) \sigma_{d s, D}\left(\varphi-\varphi^{\prime} \mid \varphi^{\prime}\right) d \varphi^{\prime}+ \\
& +\int_{0}^{\varphi} C_{U}\left(\varphi^{\prime}\right) G_{s s, U}\left(\varphi-\varphi^{\prime} \mid \varphi^{\prime}\right) \sigma_{s s, U}\left(\varphi-\varphi^{\prime} \mid \varphi^{\prime}\right) d \varphi^{\prime}+ \\
& +\Psi_{U}(\varphi),
\end{aligned}
$$


where $\Psi_{U}(\varphi)$ is the external source of electrons from the plate $U$.

$$
\begin{aligned}
& C_{D}(\varphi)= \\
& =\int_{0}^{\varphi} C_{D}\left(\varphi^{\prime}\right) G_{s s, D}\left(\varphi-\varphi^{\prime} \mid \varphi^{\prime}\right) \sigma_{s s, D}\left(\varphi-\varphi^{\prime} \mid \varphi^{\prime}\right) d \varphi^{\prime}+ \\
& +\int_{0}^{\varphi} C_{U}\left(\varphi^{\prime}\right) G_{d s, U}\left(\varphi-\varphi^{\prime} \mid \varphi^{\prime}\right) \sigma_{d s, U}\left(\varphi-\varphi^{\prime} \mid \varphi^{\prime}\right) d \varphi^{\prime}+ \\
& +\Psi_{D}(\varphi),
\end{aligned}
$$

where $\Psi_{D}(\varphi)$ is the external source of electrons from the plate $D$.

The functions $\sigma(\tau \mid \varphi)$, with $\tau=\varphi-\varphi^{\prime}$ are equal to the SEY function at impact velocity $v_{i}\left(\tau, \varphi_{s}\right)$, thus $\sigma\left(\tau \mid \varphi_{s}\right)=\sigma\left[v_{i}\left(\tau, \varphi_{s}\right)\right]$. The impact velocity is given by $v_{i}\left(\tau, \varphi_{s}\right)=v_{\omega} \dot{\xi}\left(\tau, \varphi_{s}\right)$, using (4.65) with initial velocity $u$ given by (4.71). Again the $\sigma\left(\tau \mid \varphi_{s}\right)$ functions need to be particularized for each kind of interaction and starting plate

$$
\begin{array}{c|l}
\sigma_{d s, D}(\tau \mid \varphi)=\sigma\left[v_{i}\left(\tau, \varphi_{s}, \chi, \mathrm{E}, \Omega, \Phi\right)\right] & \begin{array}{l}
\chi=\lambda \\
\varphi_{s}=\bmod (\varphi ; 2 \pi)
\end{array} \\
\sigma_{d s, U}(\tau \mid \varphi)=\sigma\left[v_{i}\left(\tau, \varphi_{s}, \chi,-\mathrm{E}, \Omega, \Phi\right)\right] & \begin{array}{l}
\chi=\lambda \\
\varphi_{s}=\bmod (\varphi ; 2 \pi)
\end{array} \\
\sigma_{s s, D}(\tau \mid \varphi)=\sigma\left[v_{i}\left(\tau, \varphi_{s}, \chi, \mathrm{E}, \Omega, \Phi\right)\right] & \begin{array}{l}
\chi=0 \\
\varphi_{s}=\bmod (\varphi ; 2 \pi)
\end{array} \\
\sigma_{s s, U}(\tau \mid \varphi)=\sigma\left[v_{i}\left(\tau, \varphi_{s}, \chi,-\mathrm{E}, \Omega, \Phi\right)\right] & \begin{array}{l}
\chi=0 \\
\varphi_{s}=\bmod (\varphi ; 2 \pi) .
\end{array}
\end{array}
$$

The SEY function with respect to the impact velocity, $\sigma\left(v_{i}\right)$, can be any of the available ones in literature. Here, the widely used Vaughan model [Vau89] has been used, with a modification to take into account low energy electron interactions $\left[\mathrm{VMW}^{+} 05 \mathrm{~b}\right]$.

Equations (4.74) and (4.75) constitute a system of Volterra integral equations of the second kind with difference kernel, similar to those of Ref. $\left[\mathrm{AVG}^{+} 10\right]$. This system has a non-trivial solution only when $\Psi_{U}(\varphi)+\Psi_{D}(\varphi)>0$ and $\Psi_{U}(\varphi) \geq 0, \Psi_{D}(\varphi) \geq 0$ (see chapter 
9.3 of Ref. [PM98]). These functions set the initial conditions of the problem.

The remaining definitions of Table 4.10 can be derived as done in Ref. $\left[\mathrm{AVG}^{+} 10\right]$. The impact rates are

$$
\begin{aligned}
& I_{U}(\varphi)=\int_{0}^{\varphi} C_{D}\left(\varphi^{\prime}\right) G_{d s, D}\left(\varphi-\varphi^{\prime} \mid \varphi^{\prime}\right) d \varphi^{\prime}+ \\
& +\int_{0}^{\varphi} C_{U}\left(\varphi^{\prime}\right) G_{s s, U}\left(\varphi-\varphi^{\prime} \mid \varphi^{\prime}\right) d \varphi^{\prime}
\end{aligned}
$$

and

$$
\begin{aligned}
& I_{D}(\varphi)=\int_{0}^{\varphi} C_{D}\left(\varphi^{\prime}\right) G_{s s, D}\left(\varphi-\varphi^{\prime} \mid \varphi^{\prime}\right) d \varphi^{\prime}+ \\
& +\int_{0}^{\varphi} C_{U}\left(\varphi^{\prime}\right) G_{d s, U}\left(\varphi-\varphi^{\prime} \mid \varphi^{\prime}\right) d \varphi^{\prime} .
\end{aligned}
$$

Finally, the number of electrons at phase $\varphi$ is

$$
N(\varphi)=\int_{0}^{\varphi} C_{U}\left(\varphi^{\prime}\right)+C_{D}\left(\varphi^{\prime}\right)-I_{U}\left(\varphi^{\prime}\right)-I_{D}\left(\varphi^{\prime}\right) d \varphi^{\prime} .
$$

The definitions of the instantaneous SEY and order given in Ref. $\left[\mathrm{AVG}^{+} 10\right]$ are also very useful to characterize the multipactor discharge. The instantaneous SEY is defined as

$$
\sigma_{i}(\varphi)=\frac{C_{(\varphi)}}{I(\varphi)}
$$

where $C(\varphi)=C_{U}(\varphi)+C_{D}(\varphi)$ and $I(\varphi)=I_{U}(\varphi)+I_{D}(\varphi)$. The 
instantaneous multipactor order is in turn

$$
\begin{aligned}
n_{i}(\varphi) & =\frac{1}{\pi I(\varphi)} \\
& {\left[\int_{0}^{\varphi}\left(\varphi-\varphi^{\prime}\right) C_{D}\left(\varphi^{\prime}\right) G_{d s, D}\left(\varphi-\varphi^{\prime} \mid \varphi^{\prime}\right) d \varphi^{\prime}+\right.} \\
& +\int_{0}^{\varphi}\left(\varphi-\varphi^{\prime}\right) C_{U}\left(\varphi^{\prime}\right) G_{d s, U}\left(\varphi-\varphi^{\prime} \mid \varphi^{\prime}\right) d \varphi^{\prime}+ \\
& +\int_{0}^{\varphi}\left(\varphi-\varphi^{\prime}\right) C_{D}\left(\varphi^{\prime}\right) G_{s s, D}\left(\varphi-\varphi^{\prime} \mid \varphi^{\prime}\right) d \varphi^{\prime}+ \\
& \left.+\int_{0}^{\varphi}\left(\varphi-\varphi^{\prime}\right) C_{U}\left(\varphi^{\prime}\right) G_{s s, U}\left(\varphi-\varphi^{\prime} \mid \varphi^{\prime}\right) d \varphi^{\prime}\right] .
\end{aligned}
$$

\subsubsection{Numerical Simulations}

The full-wave electromagnetic solver tool FEST3D $\left[\mathrm{VMW}^{+} 05 \mathrm{a}\right.$, $\mathrm{AVR}^{+} 08$ ] has been employed for the numerical simulations. FEST3D incorporates a multipactor module consisting of a full-3D particle tracker which solves the electron paths with the leap-frog algorithm. The fields are computed over unstructured 3D tetrahedral meshes, with the integral equation technique and the Method of Moments. Therefore, FEST3D is able to simulate arbitrary 3D structures with inhomogeneous electromagnetic field distributions including multiple carrier excitation. It uses the SEY model of Ref. [Vau89, VMW ${ }^{+} 05 \mathrm{~b}$ ] and allows the user to introduce custom SEY parameters.

However, in order to compare the results with the present theory, the simulations have been restricted to $1 \mathrm{D}$ electron motion and homogeneous electric field. the time step has been chosen in order to have 240 discrete time points per period of the rf signal (considering the average frequency of the multi-carrier signal). The gridding ensures 10 divisions along the gap between the plates.

The FEST3D multipactor module computes the electron trajectories, determines all electron impacts (both single and double surface), and records the associated flight time and SEY for each one. The simulations have been done for a number of 50000 initial parti- 
cles, to have enough samples for a good statistical analysis. During the simulation, the particles can be created or destroyed depending on the impact energy. The results have been normalized to have one initial electron, in order to compare with the analytical results.

The numerical equivalent SEY is the average SEY for all impacts:

$$
\sigma_{e q}=\frac{\sum_{i=1}^{i=N_{t}} \sigma_{i}}{N_{t}}
$$

where $N_{t}$ is the total number of impacts, and $\sigma_{i}$ is the secondary emission yield at impact $i$, respectively. The numerical equivalent multipactor order is defined as the average travel time, divided by half period of the rf signal,

$$
n_{e q}=2 f \frac{\sum_{i=1}^{i=N_{t}} t_{i}}{N_{t}}
$$

where $t_{i}$ is the flight time of the electron corresponding to impact $i$.

\subsubsection{Results}

In order to validate the theory in the most general possible way, a wide range of combination of parameters has been conceived, resulting in three set of results with three different phase configurations: zero phase, triangular and arbitrary phasing.

All the simulations and analytical results have been carried out assuming a Maxwellian distribution for the electron initial velocity and $W_{t}=1.5 \mathrm{eV}$ (see 4.73). The SEY model in Ref. [VMW ${ }^{+} 05 \mathrm{~b}$ ] has been used. The single carrier threshold $V_{S C}$ has been calculated in all cases for the average frequency of the train of carriers, using the single carrier non-stationary theory $\left[\mathrm{AVG}^{+} 10\right]$. In all cases, the results have been plotted not for the whole signal period $T$ but for few relative periods of the envelope, $\tilde{T}$. This has been done for the sake of clarity and because the results have shown a repetitive pattern of period $\tilde{T}$ and, therefore, few relative periods are representative of the full discharge behaviour.

\subsubsection{Zero phases - uniform frequency spacing}

Simulations have been performed for a 6 C-band carrier signal with uniform frequency spacing of $100 \mathrm{MHz}$, zero phase, gap of $d=$ 
$0.43 \mathrm{~mm}$, equal amplitude $E_{0}$, and assuming ECSS [EST03] Silver parameters for the material. The signal parameters are those employed in Table 4.9 of Section 4.4.3.2 and the SEY parameters are given in Table 4.11. The multi-carrier signal employed in these tests have a period of $T=100 \mathrm{~ns}$ and $T=10 \tilde{T}$.

\begin{tabular}{cccc}
$W_{1}(\mathrm{eV})$ & $W_{m}(\mathrm{eV})$ & $\sigma_{m}(\mathrm{eV})$ & $\sigma_{0}(\mathrm{eV})$ \\
\hline 30 & 165 & 2.22 & 0.5 \\
\hline
\end{tabular}

Table 4.11.

SEY parameters for ECSS Silver [EST03].
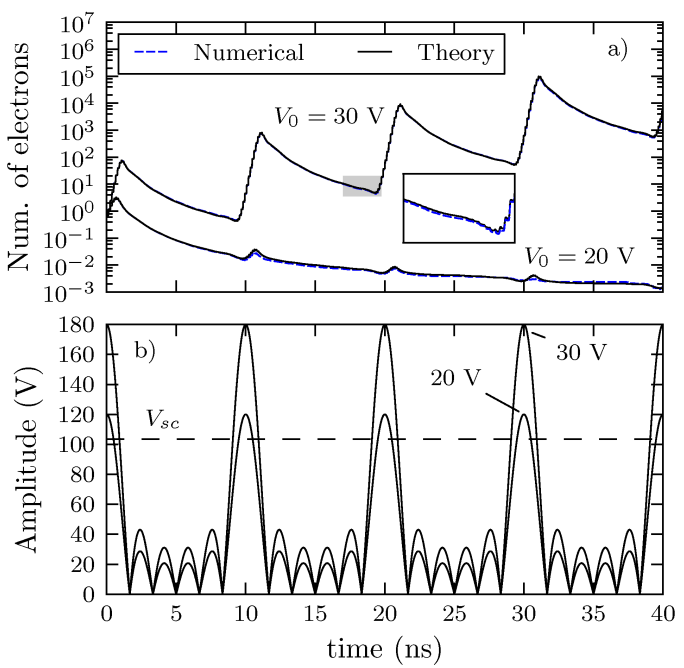

Figure 4.33.

(Color online) Time evolution of electron population (a) and signal envelope (b) for $V_{0}=$ $30 \mathrm{~V}$ and $V_{0}=20 \mathrm{~V}$ for the parameters defined in Section 4.4.5.1. The inner window represents the enlargement of the shaded area.

Fig. 4.33 (a) shows the time evolution of a multipactor discharge given by (4.79), whereas Fig. 4.33 (b) depicts the envelope of the multi-carrier signal given by (4.51). Two different scenarios are considered. First, a case with an amplitude per carrier of $V_{0}=E_{0} d=$ $30 \mathrm{~V}$, and second a case with an amplitude per carrier of $V_{0}=20 \mathrm{~V}$. As it can be seen, these two values have been chosen, for this particular case, to illustrate an overall electron growth (causing a multipactor discharge) and an overall electron absorption, respectively.

It is remarkable that, as expected, in both scenarios the electron population increases when the envelope is above the single carrier 


\section{Figure 4.34.}

(Color online) Electron impact rate (a) and envelope (b) for $V_{0}=$ $30 \mathrm{~V}$ signal and the parameters defined in Section 4.4.5.1.
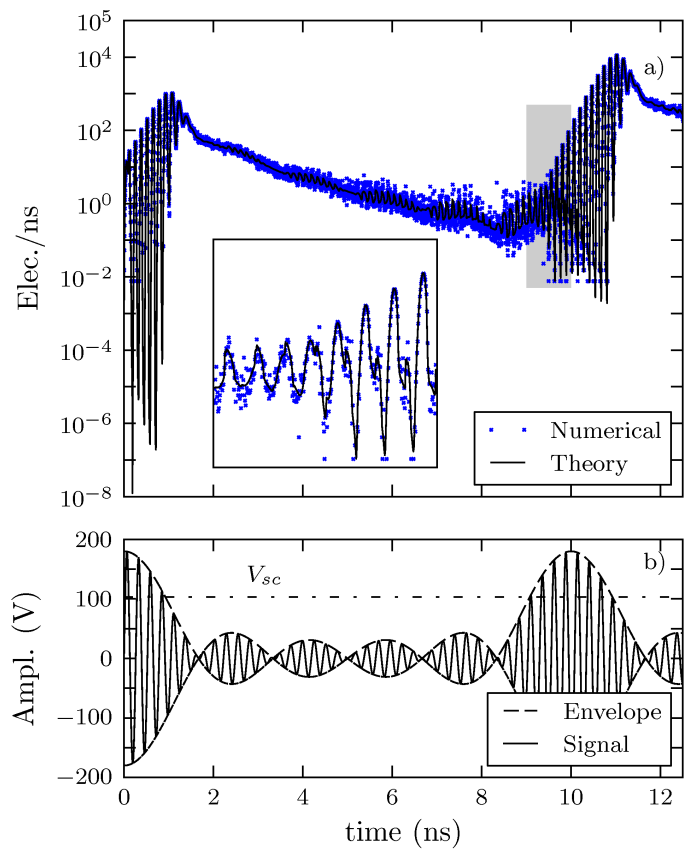

threshold, $V_{S C}$, and decreases when it is below (see the correspondance in time between plots (a) and (b) of Fig 4.33). However, the $V_{0}=30 \mathrm{~V}$ case ensures a higher electron creation than absorption (in Fig. 4.33 (a) the peaks are higher than the valleys), and therefore the electron population increases indefinitely in time, in what is called a long-term multipactor discharge $\left[\mathrm{AVG}^{+} 07\right]$. In the second scenario, $V_{0}=20 \mathrm{~V}$, even if its respective envelope is above $V_{S C}$ during a short interval, the absorption of the electrons is higher than their creation (in Fig 4.33 (a) the peaks are lower than the valleys), and therefore the number of electrons decreases in time. The theoretical and numerical results match so precisely that they practically overlap.

Figures 4.34 and 4.35 show other statistics predicted by the theory: impact rate (4.77 - 4.78), instantaneous SEY (4.80) and instantaneous order (4.81), showing an excellent agreement as well. Only one period of the $V_{0}=30 \mathrm{~V}$ envelope is represented. It can be seen that the results are synchronized with the multi-carrier signal, following 

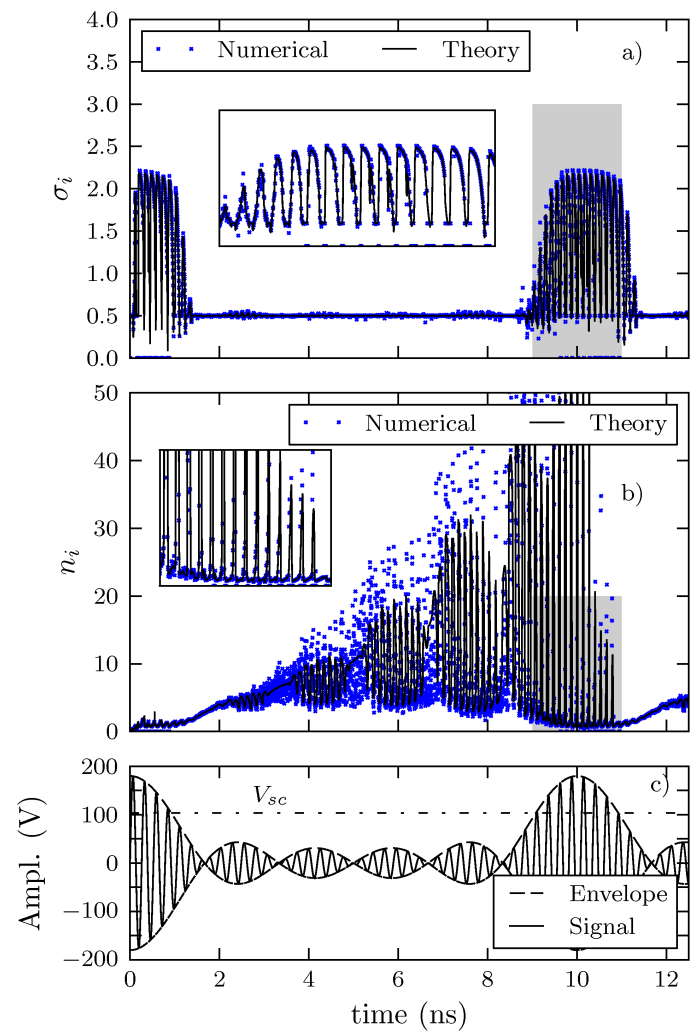

Figure 4.35.

Color online) Instantaneous SEY (a) instantaneous order (b) and envelope (c) for $V_{0}=$ $30 \mathrm{~V}$ signal and the parameters defined in Section 4.4.5.1. The inner window represents the enlargement of the shaded area.

both the faster oscillations corresponding to the instantaneous frequency and the slower periodic variations of the envelope. The end of each period of the envelope (around $t=9 \mathrm{~ns}$ in this example) corresponds to a low value of the amplitude and, therefore, to a valley in the electron population. Thus, in the numerical simulations done with FEST3D, at the end of each period, the number of available electrons to perform the statistics is lower than at the beginning. This explains the higher dispersion of the numerical results at the end of the period, present in both figures. This is not the case for the non-stationary multipactor theory, where no dispersion is seen. 


\subsubsection{Triangular phases - uniform frequency spacing}

Simulations have been carried out for an $8 \mathrm{Ku}$-band carrier signal with uniform frequency spacing of $38.3 \mathrm{MHz}$, triangular phasing, equal amplitude $E_{0}$, gap of $d=0.42 \mathrm{~mm}$ and assuming ECSS [EST03] Alodine parameters for the material. The signal parameters are those employed in Table 4.12 and the SEY parameters are given in Table 4.13. This particular frequency scheme yield a period of the envelope of $T=10 \mu$ s and $T=383 \tilde{T}$.

Table 4.12.

8 carrier signal parameters.

\begin{tabular}{ccc} 
carrier & frequency $(\mathrm{GHz})$ & phase $(\mathrm{deg})$ \\
\hline 1 & 11.8589 & 0 \\
2 & 11.8972 & 51.111 \\
3 & 11.9355 & 102.223 \\
4 & 11.9738 & 153.334 \\
5 & 12.0121 & 153.334 \\
6 & 12.0504 & 102.223 \\
7 & 12.0887 & 51.111 \\
8 & 12.127 & 0 \\
\hline
\end{tabular}

Table 4.13.

SEY parameters for ECSS Alodine [EST03].

\begin{tabular}{cccc}
$W_{1}(\mathrm{eV})$ & $W_{m}(\mathrm{eV})$ & $\sigma_{m}(\mathrm{eV})$ & $\sigma_{0}(\mathrm{eV})$ \\
\hline 41 & 180 & 1.83 & 0.5
\end{tabular}

Fig. 4.36 shows the time evolution of a multipactor discharge given by (4.79) (a) and the envelope of the multi-carrier signal given by (4.51)(b) for two different scenarios. First, a case with an amplitude per carrier of $V_{0}=E_{0} d=140 \mathrm{~V}$, and second, a case with an amplitude per carrier of $V_{0}=100 \mathrm{~V}$.

In this case, the envelope of the signal presents a wider main lobe than the zero phase signal of Section 4.4.5.1, which also implies longer intervals of electron creation and shorter intervals of electron absorption. The scenarios have been chosen in order to produce a long-term multipactor discharge for $V_{0}=140 \mathrm{~V}$ and an overall electron absorption for $V_{0}=100 \mathrm{~V}$. The theoretical and numerical results match perfectly for the initial instants of the discharge, and suffer a pro- 


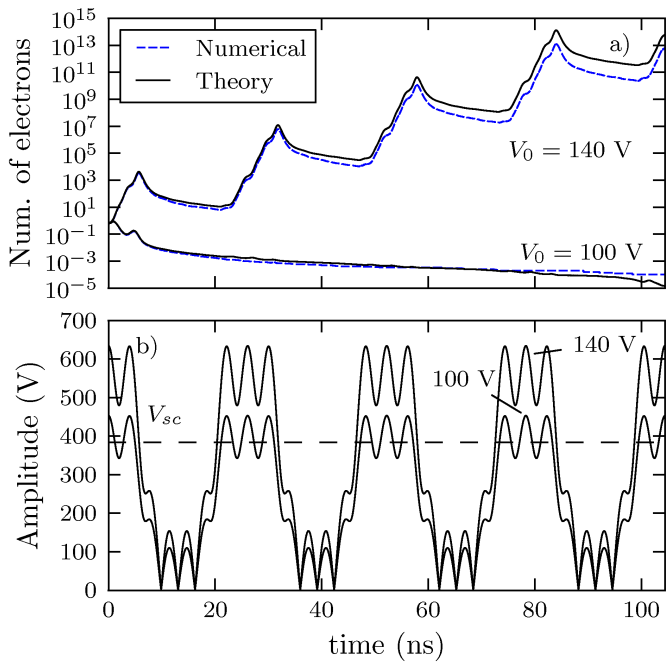

Figure 4.36.

(Color online) Time evolution of electron population (a) and signal envelope (b) for $V_{0}=$ $140 \mathrm{~V}$ and $V_{0}=1000$ $\mathrm{V}$ for the parameters defined in Section 4.4.5.2.

gressive offset at the end of the simulation due to the accumulation of numerical error. This error is more evident here than in the previous results of Section 4.4.5.1 because the frequency of the carriers is higher, as well as the frequency-gap product. This implies longer electron transition times and, therefore, longer tails in the TTPD functions which need to be truncated when numerically integrated in $(4.74,4.75)$. However, the behavior of the electron population remains equal for both the theoretical and numerical results.

\subsubsection{Arbitrary phases - non-uniform frequency spacing and different amplitudes}

Simulations have been carried out for an $8 \mathrm{Ku}$-band carrier signal with non-uniform frequency spacing, arbitrary phasing, arbitrary amplitudes, gap of $d=1 \mathrm{~mm}$ and assuming ECSS [EST03] Copper parameters for the material. The signal parameters are those employed in Table 4.14 (the given amplitudes are relative to the first carrier), and the SEY parameters are given in Table 4.15.

Fig. 4.37 shows the time evolution of a multipactor discharge given by (4.79) (a) and the envelope of the multi-carrier signal given by (4.51)(b) for two different scenarios. First, a case with an ampli- 
Table 4.14. 8 carrier signal parameters. The given amplitudes are relative to the first carrier

\begin{tabular}{cccc} 
carrier & frequency $(\mathrm{GHz})$ & phase $(\mathrm{deg})$ & amplitude $(\mathrm{V})$ \\
\hline 1 & 11.852 & 227.3 & 1 \\
2 & 11.892 & 127.83 & 0.2494 \\
3 & 11.932 & 358.92 & 4.0039 \\
4 & 11.972 & 80.70 & 1.9236 \\
5 & 12.012 & 234.88 & 3.6576 \\
6 & 12.072 & 217.7 & 2.1253 \\
7 & 12.132 & 139.41 & 3.4734 \\
8 & 12.192 & 51.19 & 0.1199 \\
\hline
\end{tabular}

Table 4.15.

SEY parameters for ECSS Copper [EST03].

\begin{tabular}{cccc}
$W_{1}(\mathrm{eV})$ & $W_{m}(\mathrm{eV})$ & $\sigma_{m}(\mathrm{eV})$ & $\sigma_{0}(\mathrm{eV})$ \\
\hline 25 & 175 & 2.25 & 0.5 \\
\hline
\end{tabular}

tude per carrier of $V_{0}=E_{0} d=140 \mathrm{~V}$, and second, a case with an amplitude per carrier of $V_{0}=100 \mathrm{~V}$.

For this arbitrary distribution of phases, the envelope of the signal does not present a clear structure, but rather a noisy behavior, with a period of $T=250 \mathrm{~ns}$ and $T=5 \tilde{T}$. As it was done for the previous results, the scenarios have been chosen in order to produce a longterm multipactor discharge for $V_{0}=140 \mathrm{~V}$ and an overall electron absorption for $V_{0}=100 \mathrm{~V}$. In spite of the small numerical offset at the end of the simulation, the theoretical and numerical results match quite well again.

\subsubsection{Conclusions}

A new theory for multipactor with multi-carrier signals in parallel plates based on the statistical non-stationary multipactor theory $\left[\mathrm{AVG}^{+} 10\right]$ has been proposed. This new theory models the real dynamics of the electrons during the multipactor discharge for both single and double surface interactions, calculating the time evolution of the charge growth, electron absorption and creation rates, as well as the instantaneous SEY and order. It is valid for multi-carrier signals with an arbitrary number of carriers, arbitrary frequency and 


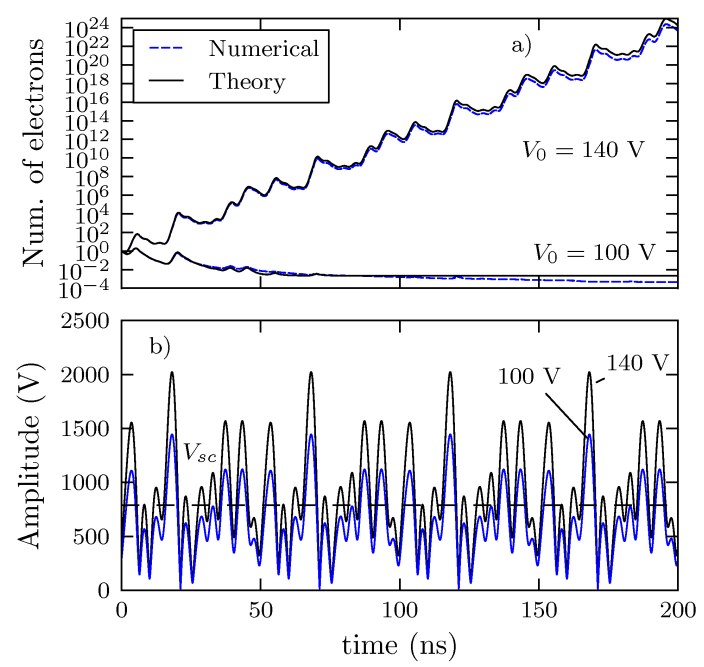

Figure 4.37.

(Color online) Time evolution of electron population (a) and signal envelope (b) for $V_{0}=$ $140 \mathrm{~V}$ and $V_{0}=100 \mathrm{~V}$ for the parameters defined in Section 4.4.5.3.

phase, and for any material (as long as it can be characterized by an analytical SEY function).

An extensive set of numerical tests has been carried out to validate the theory under many different conditions. The numerical and theoretical results match almost perfectly for all cases, which reveals that the theory successfully predicts the multipactor discharge for the general multi-carrier case.

To the authors' knowledge, this is the first theory of multipactor for multi-carrier signals that completely characterizes the phenomenon, which has been successfully verified by numerical simulations. This theory establishes a new framework for the study of the multipactor process for multi-carrier signals, which can be extended in future works to more general types of non-stationary time varying signals and different geometries. 
4.5. Prediction of Multipactor Breakdown for Multi-carrier

Applications: The Quasi-stationary Method

\subsection{Prediction of Multipactor Breakdown for Multi-carrier Applications: The Quasi-stationary Method}

\subsubsection{Introduction}

Multipactor, also known as multipactoring or multipaction, is an electron avalanche-like discharge occurring in microwave devices operating at high power levels and in vacuum or near vacuum condition [GvE48, HW54, Vau88]. When initially discovered, it was studied as a beneficial effect for signal amplification in cold-cathode tube for TV applications by P. Farnsworth [Far34], who originally coined the name of multipactor. Nowadays, multipactor is considered as a dangerous collateral effect in high-power vacuum applications, which must be avoided.

The phenomenon occurs when initial free electrons (primary) are accelerated by the RF fields, and impact against the device walls with enough energy to extract more electrons (secondary) from the surface. If the resulting electronic bunch enters in resonance with the field, this process repeats itself until the electron density reaches a certain level to produce noticeable disturbance of the signal, such as distortion, additive noise or reflection, and ultimately produces a destructive discharge that can even damage the device. In operation, primary electrons come from different sources such as field emission or electron cascades produced by cosmic rays [Ste57]. In the laboratory, in order to induce the discharge for multipaction testing purposes, different electron seeding techniques are available, such as radioactive sources, controlled electron beams or photoelectric effect [EST03]. Multipactor may appear in many types of components, such as passive or active high power devices in guided or microstrip technologies and antennas. Thus, it affects different industry sectors such as satellite communications [CMK07] or particle accelerators [GGC $\left.{ }^{+} 05\right]$.

The biggest effort of the multipactor research lines is devoted to the study and characterization of the phenomenon in order to predict under which conditions it will appear, and thus design multipactorfree components. Traditionally, multipactor has been studied for single-carrier signals. The single-carrier prediction techniques are 
usually based on the multipactor theory, for which there are abundant references (see for example [GvE48, HW54, Vau88]), and 2D or 3D numerical Particle-in-cell codes (PIC) $\left[\mathrm{VMW}^{+} 05 \mathrm{a}, \mathrm{FES}, \mathrm{SPA}, \mathrm{CST}\right]$, which combine electromagnetic solvers and electron trackers. Given some input parameters, such as the frequency of operation, device dimensions and material Secondary Emission Yield (SEY) properties, these single-carrier prediction methods provide the threshold for the multipactor breakdown power. The predicted thresholds are used by the industry to design and assess the margins of operated power in the device to be multipactor-free.

Nevertheless, realistic satellite communication systems combine more than one channel in a single output, what is called a multicarrier signal. The multi-carrier signal combines the transmission power of the individual channels. Its amplitude is time varying and depends on the relative amplitudes and phases of the channel carriers. Therefore, in the multi-carrier path of the spacecraft (after multiplexing the channels) extremely high peak power levels may be attained, thus increasing the risk of a multipactor discharge [GW96, RLR ${ }^{+} 94$ ].

By the time of speaking, the theory for multipactor and multicarrier signals is rather scarce. To the authors' knowledge, the only existing full theory for multi-carrier operation is provided in $\left[\mathrm{AMV}^{+} 11\right]$. Numerical solvers capable of handling multi-carrier signals exist as well $\left[\mathrm{AVR}^{+} 08, \mathrm{ECS}, \mathrm{FES}\right]$. However, in the multi-carrier case there are many more parameters involved in the multipactor discharge than for the single-carrier case, which include the carrier frequency spacing, the relative phases among the carriers and the amplitude (or power) per carrier. Therefore, the current multipactor theory and numerical software tools for multi-carrier signals are able to determine if there is multipactor discharge for a fixed configuration. However, they do not provide the worst case, which is the combination of all the variables of the problem that produces a multipactor discharge with the minimum power per carrier. Thus, the current multi-carrier theory/software tools do not predict the lowest multipactor breakdown level.

The design rules that are currently being applied by the space industry are based on simplifications that allow applying the singlecarrier predictions to the multi-carrier case. The most restrictive one is the $K^{2}, K$ being the number of carriers, which takes the peak 
4.5. Prediction of Multipactor Breakdown for Multi-carrier Applications: The Quasi-stationary Method

power of the multi-carrier signal as the $\mathrm{CW}$ power of an equivalent single-carrier signal. The multipactor breakdown is then equal to $P_{s c} / K^{2}$ per carrier, where $P_{s c}$ is the single-carrier breakdown that can be calculated with single-carrier predictors [EST03]. This design rule is known to be very conservative and typically gives much lower breakdown power predictions than measured ones. This imposes unnecessary constraints on the design, and usually forces to carry out cumbersome test campaigns to validate the components.

The first attempt for trying to reduce the margins is the 20-gapcrossing rule (20GCR) [WJ89, EST03], which establishes a more relaxed criterion of multipactor: It can only appear when the multicarrier signal envelope is above the single-carrier threshold for a time such that an electron crosses the gap 20 times. In other words, the 20GCR rule allows the multi-carrier signal to be above the threshold for a short time, assuming that the electron build-up will not be enough to produce a discharge. Equivalently, the above $K^{2}$ rule would be the zero-gap-crossing rule, i.e. it does not allow any electron crossing (impact) above the threshold. With respect to the $K^{2}$ rule, the 20GCR predicts higher multipactor thresholds and reduces the design constraints.

However, the 20GCR rule is based only on the study of numerical simulations and measurements, and does not have a solid physical basis. The question that naturally arises is why 20 and not another value, and why 20 should be a universal value valid for all kind of signals and devices. This uncertainty on the prediction rule implies large safety margins that are imposed to the predicted values [EST03]. As a consequence, the 20-gap-crossing rule, although being more relaxed than the $K^{2}$ rule, still yields very conservative predictions in most cases.

This work proposes a novel Quasi-Stationary (QS) prediction technique for multipactor in multi-carrier signals, with the aim of giving more accurate predictions in order to reduce the safety margins, avoid unnecessary design constraints and reduce the test campaigns as much as possible. Even if a full multi-carrier theory is already available $\left[\mathrm{AMV}^{+} 11\right]$, the new technique presented in this paper is still based on the single-carrier theory, following a similar approach as the previous ones. However, it takes more sophisticated simplifications on the multi-carrier signal and employs a new electron growth model 
(presented in Section 4.5.3). By applying the single-carrier theory, the number of parameters of the problem reduces significantly and allows for more simple and intuitive solutions.

The QS prediction method is based on the non-stationary theory for single-carrier signals $\left[\mathrm{AVG}^{+} 10\right]$, which belongs to the family of statistical theories that introduce the randomness of electron emission velocity and angle. In spite of their complexity, the statistical theories have the advantage of matching better the experimental results $\left[\mathrm{SSV08}, \mathrm{AVG}^{+} 10\right]$. Among the statistical theories, the nonstationary one is able to model both the electron growth and absorption, above and below the multipactor threshold, and it considers both single-surface and double-surface interactions. In addition, it gives analytical expressions for the instantaneous SEY and multipactor order. Therefore, the non-stationary theory becomes the most suitable one for multipactor prediction with multi-carrier signals.

Within this work, a number of samples in waveguide technology has been manufactured and tested in order to assess the prediction accuracy of the new QS tool and the current 20GCR. In Section 4.5.2 some background on multipactor is given, including a review of the 20GCR. The QS model is presented in Section 4.5.3. Section 4.5.4 gives some details on the manufactured samples and the test setup. The predictions and experimental results are presented in Section 4.5.6. Finally, Section 4.5.7 offers some conclusions.

\subsubsection{Background}

\subsubsection{Multipactor in multi-carrier signal}

A multi-carrier signal $V(t)$, composed of $K$ carriers with amplitudes $V_{i}$, angular frequencies $\omega_{i}$, and phases $\phi_{i}, i=1, \ldots, K$, has the form

$$
V(t)=\sum_{i=1}^{K} V_{i} \cos \left(\omega_{i} t+\phi_{i}\right)
$$

According to $\left[\mathrm{AMV}^{+} 11\right]$, the expression above can be alternatively expressed as a modulated signal with envelope

$$
V_{e}(t)=\sqrt{\left[\sum_{i=1}^{K} V_{i} \cos \left(\omega_{i} t+\phi_{i}\right)\right]^{2}+\left[\sum_{i=1}^{K} V_{i} \sin \left(\omega_{i} t+\phi_{i}\right)\right]^{2}},
$$


4.5. Prediction of Multipactor Breakdown for Multi-carrier Applications: The Quasi-stationary Method

The envelope is periodic, and its period $T$ can be computed by finding the greatest common divisor ( $\mathrm{gcd}$ ) of the differences between the signal frequencies $\left(f_{i}=\omega_{i} / 2 \pi\right)$ and the lowest one $\left(f_{1}\right)$.

$$
T=1 / f, \quad f=\operatorname{gcd}\left(f_{2}-f_{1}, f_{3}-f_{1}, \ldots, f_{K}-f_{1}\right)
$$

For a multi-carrier signal with a specific set of frequencies, its envelope will have a fixed period, but its shape will vary in accordance with the choice of the phase and amplitude of each carrier. The shape may be seen as a set of periodic lobes. In general, the height of such lobes is related to its width in such a way that the higher the envelope is, the narrower the lobes are. Theoretically, for equal amplitude, $V_{i}=V_{0}, \forall i$, the multi-carrier signal envelope is comprised between two limit values, $K V_{0}$ corresponding to the in-phase scheme (all carriers have the same relative phase), and $\sqrt{K} V_{0}$ for a totally uncorrelated phase scheme (where the lobes spread and overlap to form a flatter envelope). There are different boundary models that relate the height and the width of the envelope, such as Wolk [WSS00] or Angevain [ADDM09] boundary functions. These provide the voltage factor $F_{v}$, which relates the boundary level and the level per carrier, for each envelope width, $\Delta T$

$$
V_{e}(\Delta T)=F_{v}(\Delta T) V_{0}
$$

Fig.4.38 shows an example for different phase schemes for a signal with $K=8$ and $V_{0}=1$ with a uniform frequency spacing $\Delta f=$ $f_{i+1}-f_{i}=40 \mathrm{MHz}, \quad \forall i$.

As it is explained in $\left[\mathrm{AMV}^{+} 11\right]$, the instantaneous frequency is also periodic with the same period of the envelope and with an oscillating value around the mean frequency of all carriers. Therefore, the frequency of the multi-carrier signal can be approximated as a constant value equal to the mean frequency of all carriers, i.e. $f_{m}$.

The study of the multipactor phenomenon in multi-carrier signals is rather more complicated than for the single-carrier case. Conceptually, the process can be described as follows. When the multi-carrier signal envelope, $V_{e}(t)$, surpasses a certain level, the electrons are accelerated with enough energy to initiate a multipactor discharge and, thus, the electron population increases. The value of such a threshold is not well known. However, there are evidences that indicate that it 


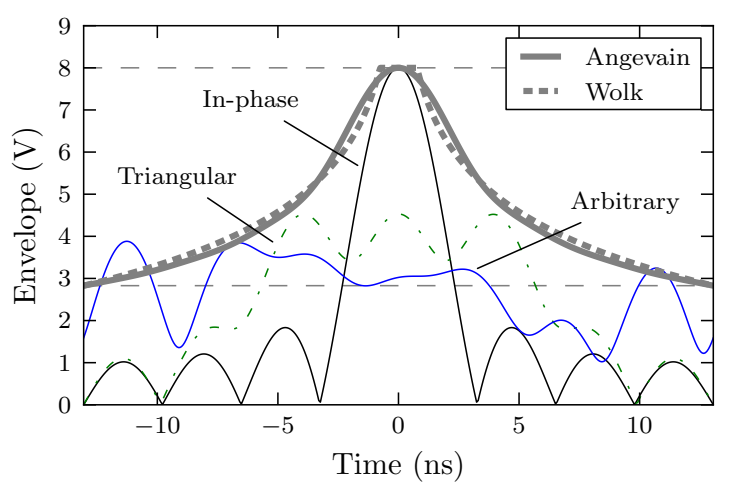

Figure 4.38.

Multi-carrier signal envelopes for different phase schemes with $K=8$ and $V_{i}=V_{0}=1, \quad \forall i$ with a uniform frequency spacing $\Delta f=40 \mathrm{MHz}$. Angevain's and Wolk's boundary functions are also plotted. $K V_{0}$ and $\sqrt{K} V_{0}$ limits are represented by dotted horizontal lines.

must be close to the breakdown threshold in the single-carrier case, $V_{S C}$, for a frequency equal to the mean frequency of all carriers, $f_{m}$, as $\left[\mathrm{AVG}^{+} 10\right]$ suggests. On the other hand, when $V_{e}(t)$ is below $V_{S C}$, the electrons impact on the device walls with low energies, implying a SEY below one, and the electrons being therefore absorbed.

The intervals in which $V_{e}(t)$ is above $V_{S C}$ are called "on" intervals, and those where it is below are known as "off" intervals $\left[\mathrm{AVG}^{+} 07\right]$. Since the envelope is periodic, "on" and "off" intervals are alternated indefinitely in time. Hence, there will be a multipactor discharge in two cases: Either the "on" interval is long enough to make the electron population grow to a detectable level in the first period of the envelope, which is called a single-event discharge, or the electron growth during the "on" interval is higher than the electron absorption during the "off" interval. This makes the electron population grow slowly, period after period, culminating in a long-term multipactor discharge $\left[\mathrm{AVG}^{+} 07, \mathrm{AMA}^{+} 10\right]$. Fig. 4.39 shows an example of a long-term multipactor discharge with an in-phase multi-carrier signal, extracted from $\left[\mathrm{AMV}^{+} 11\right]$. The long-term discharge build-up is typically in the range of few nanoseconds and the multipactor discharges are in general not self-sustained. Therefore, in practice, both kind of discharges are indistinguishable in laboratory. Nevertheless, each of them have different implications for the multipactor breakdown level. Long-term discharges are thought to be more restrictive than single-event ones $\left[\mathrm{AMA}^{+} 10, \mathrm{AVR}^{+} 08\right]$. 
Figure 4.39.

Example of electron growth in a longterm multipactor discharge extracted from $\left[\mathrm{AMV}^{+} 11\right]$, corresponding to a $K=6$ carrier signal with equal amplitudes, zero phase (in-phase) and a uniform frequency spacing $\Delta f=100 \mathrm{MHz}$. Shaded areas correspond to "on" intervals. The single-carrier threshold, $V_{S C}$ is marked with a horizontal dotted line.

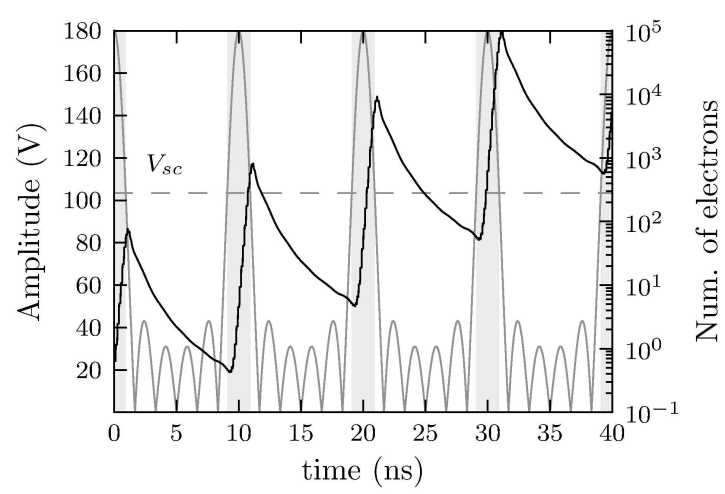

There are infinite combinations of amplitude and phases that lead to a multipactor discharge. Assuming that all carriers have equal amplitude, the worst case is defined as the combination of phases that causes a multipactor discharge with the minimum amplitude (or power) per carrier. This worst case must be the goal of any multipactor prediction method for multi-carrier signals.

\subsubsection{The 20-gap-crossing rule}

The 20-gap-crossing rule (20GCR) is very simple. It simplifies the multi-carrier envelope as a pulsed signal which can only be above ("on") or below ("off") the single-carrier threshold, $V_{s c}$. As its own name indicates, it establishes that there will be a multipactor discharge when the "on" interval is long enough to ensure at least 20 electron impacts [EST03]. In order to provide a larger margin, the $20 \mathrm{GCR}$ takes the lowest frequency of the train of carriers, $f_{1}$ (instead of $f_{m}$ ), as the reference frequency for the calculation of the singlecarrier breakdown threshold. For a multipactor discharge of order $n$ (the order of the multipactor discharge sets the number of cycles between consecutive impacts for a single electron), the "on" time is

$$
T_{20}=\frac{10 n}{f_{1}} .
$$


The rule does not give any value for the worst case phases or RF breakdown power. It just gives the length of the "on" interval. In order to find such combination of phases and power, it is necessary to conform the envelope to the desired shape through numerical optimizers, such as simulated annealing [KGV83] or genetic algorithms [PSL05], which search the right combination of phase and amplitude for each carrier ensuring $T_{20}$. Another possibility is to use boundary functions for the envelope amplitude such as [WSS00, ADDM09], which only estimates the breakdown power.

The main advantage of this rule, i.e. its simplicity, is at the same time its main drawback. It is an empirical rule and the criterion that leads to a number of 20 gap crossings it is not clear, and why such a value is applicable to all situations. This is, it does neither take into account how high the envelope with respect to the singlecarrier threshold is, nor the dependence of the multipactor order with voltage, or the kind of material in terms of the SEY curve.

For amplitudes close to the breakdown level, the higher the envelope amplitude, the higher the impact energy is, and thus, the higher the SEY $\left[\mathrm{AVG}^{+} 10\right]$. Therefore, it seems logical that for higher amplitudes the number of necessary impacts to cause a detectable discharge gets lower. For instance, for an amplitude equal to the single carrier threshold, the secondary emission yield is nearly one, which implies no electron growth (and no discharge) at all, no matter how many electron impacts occur.

On the other hand, it also seems logical that the number of gap crossings to create a discharge is different for materials with different SEY curves. For example it would be expectable that the number of gap crossings for gold would be higher than for aluminium, since gold is known to typically have a much lower secondary emission yield [EST03].

Furthermore, the 20GCR only takes into account single-event discharges and completely disregards long-term discharges.

\subsubsection{Parallel-plate geometry}

Although theories for more sophisticated geometries are available in the literature, the parallel-plate geometry is the simplest and most 
4.5. Prediction of Multipactor Breakdown for Multi-carrier

Applications: The Quasi-stationary Method

representative case for all of them, and will be used for the present analysis.

The parallel-plate model assumes a homogeneous RF electric field between the plates, which allows for equivalent voltage definition. But in real microwave applications the circuit and signal specifications are given in terms of signal power, the fields along the structure varying strongly depending on the particular geometry of the device.

In order to translate between both definitions, one may isolate the critical part of the circuit in which multipactor is expected to occur and compute the voltage at $1 \mathrm{~W}, V_{1 W}$, by means of network theory (for simple structures) or by numerical integration of the electric field along the gap employing full-wave field solvers such as [HFS, CST, FES]. Therefore, the voltage at the gap $V_{g}$, given an input power $P_{i n}$ is given by

$$
V_{g}=V_{1 W} \sqrt{P_{i n}}
$$

Of course, this is an approximation that assumes that the electric field is homogeneous along the gap, which does not occur for most practical situations. However, this is the worst case and it is still a valid and commonly used approximation for a wide range of waveguide and coaxial structures [WJ89, $\left.\mathrm{VMW}^{+} 05 \mathrm{a}, \mathrm{PSK}^{+} 03, \mathrm{EST} 03\right]$.

\subsubsection{Quasi-stationary model prediction}

The Quasi-Stationary (QS) model follows a completely different approach than the $20 \mathrm{GCR}$. It does not simplify the multi-carrier envelope as a pulsed signal, but models the electron growth considering its real time-varying shape $V_{e}(t)$. The worst case is computed searching the envelope that triggers the multipactor discharge with the lowest breakdown power per carrier among all possible shapes. Therefore, the QS model does not employ the concept of "gap-crossings" any more, since it uses a more general and powerful definition of the envelope.

Moreover, the QS model is based on the single carrier nonstationary theory $\left[\mathrm{AVG}^{+} 10\right]$. Therefore, contrarily to the classical multipactor theory, the QS model considers random electron emission velocity and non-resonant electron trajectories. Hence, the electrons follow different paths and impact at different times, with different 
energies. This scenario is valid for voltages above and below the multipactor threshold, which implies electron growth and absorption, respectively. The solutions given by this theory considerably depend on the secondary emission velocity distribution. In this work a Maxwellian energy distribution with a spread of $3 \mathrm{eV}$ has been used as in $\left[\mathrm{AVG}^{+} 10\right]$.

\subsubsection{Theory}

For any arbitrary multi-carrier signal, the electron growth can be approximated by $\left[\mathrm{AVG}^{+} 07\right]$

$$
N(t+\Delta t) \simeq N(t)\left\{1+\Delta t\left[\sigma_{a v}(t, \Delta t)-1\right] I_{a v}(t, \Delta t)\right\},
$$

where $I_{a v}(t, \Delta t)$ and $\sigma_{a v}(t, \Delta t)$ are the average impact rate and average SEY from $t$ to $t+\Delta t$ defined as

$$
I_{a v}(t, \Delta t)=\frac{2 f_{m}}{\Delta t} \int_{t}^{t+\Delta t} \frac{d t^{\prime}}{n\left(t^{\prime}\right)},
$$

and

$$
\sigma_{a v}(t, \Delta t)=\frac{1}{\Delta t} \int_{t}^{t+\Delta t} \sigma\left(t^{\prime}\right) d t^{\prime}
$$

respectively.

Here $n(t)$ and $\sigma(t)$ are the instantaneous values of the multipactor order and the SEY of discharge of time-varying amplitude $V e(t)$ at time $t$. The approximation given by (4.90) is valid in the limit of small $\Delta t$, i.e $2 f_{m} \Delta t<<1$.

By reordering some terms and setting the limit $\Delta t \rightarrow 0$, it is possible to express (4.90) in a differential equation form as

$$
\frac{d N(t)}{d t}=N(t)[\sigma(t)-1] \frac{2 f_{m}}{n(t)} .
$$

In order to solve (4.93) one needs to know $n(t)$ and $\sigma(t)$, for which there is not analytical direct expression, and can be obtained only by solving a system of Volterra integral equations for the multi-carrier case as shown in $\left[\mathrm{AMV}^{+} 11\right]$.

At this point, the QS model assumes that the multi-carrier envelope, $V_{e}(t)$, varies slowly enough to consider that, at a certain time $t$, 
4.5. Prediction of Multipactor Breakdown for Multi-carrier Applications: The Quasi-stationary Method

the multi-carrier discharge is equivalent to a single-carrier one with operation frequency $f_{m}$ and fixed amplitude $V \equiv V_{e}(t)$. This allows for using the single-carrier theory, considerably reducing the number of parameters of the problem. A reasonable limit for the validity of this assumption can be given by $f_{m} / \Delta f_{\max }>n$, where $n$ is the nominal multipactor order at breakdown level and $\Delta f_{\max }=f_{K}-f_{1}$ is the maximum frequency component of the envelope spectrum, whose inverse sets the minimum variation period. The above limit imposes that at least a number of $2 n \mathrm{RF}$ cycles (equivalent to two electron impacts) are contained in such a period.

The QS assumption allows the following approximations: First, according to $\left[\mathrm{AVG}^{+} 10\right]$, the instantaneous SEY of a single-carrier discharge is stable in time for every amplitude, $V$, with a value of $\tilde{\sigma}(V)$. Therefore, $\sigma(t)$ can be approximated by

$$
\sigma(t) \simeq \tilde{\sigma}(V) .
$$

On the other hand, the value of the instantaneous multipactor order of a single-carrier discharge is stable for voltages above the breakdown level, but not for voltages below breakdown, since the multipactor order diverges, increasing indefinitely in time $\left[\mathrm{AVG}^{+} 10\right]$. Since an analytical expression is necessary to derive the rest of the theory, in this work we propose a simple parabolic shape approximation of the form

$$
n(t) \simeq \tilde{n}\left(V, t_{r}(t)\right)=\alpha(V) t_{r}(t)^{2}+\beta(V),
$$

which provides a reasonable resemblance with the observed behavior. The values of $\alpha(V)$ and $\beta(V)$ can be obtained straightforwardly by numerical fitting of the curve for each value of $V$. The term $t_{r}(t)$ is the time elapsed since the beginning of a particular "off" interval. For an arbitrary multipactor envelope with $k$ "off" intervals where $t_{s, i}$ is the starting time, and $t_{e, i}$ is the ending time for each of them $(1 \leq i \leq k), t_{r}(t)$ is defined as

$$
t_{r}(t)= \begin{cases}t-t_{s, 1}, & t_{s, 1} \leq t<t_{e, 1} \\ t-t_{s, 2}, & t_{s, 2} \leq t<t_{e, 2} \\ \cdots & \\ t-t_{s, k}, & t_{s, k} \leq t<t_{e, k}\end{cases}
$$

The expression given by (4.95) is also valid for the region above breakdown ("on" interval), setting $\alpha(V)=0$. Fig. 4.40 shows an example 
of such fitting process. Figs. 4.41, 4.42 and 4.43 show detailed maps of the $\tilde{\sigma}(V), \alpha(V)$ and $\beta(V)$ parameters, respectively, for ECSS silver [EST03], and computed with the theory of $\left[\mathrm{AVG}^{+} 10\right]$. Then,
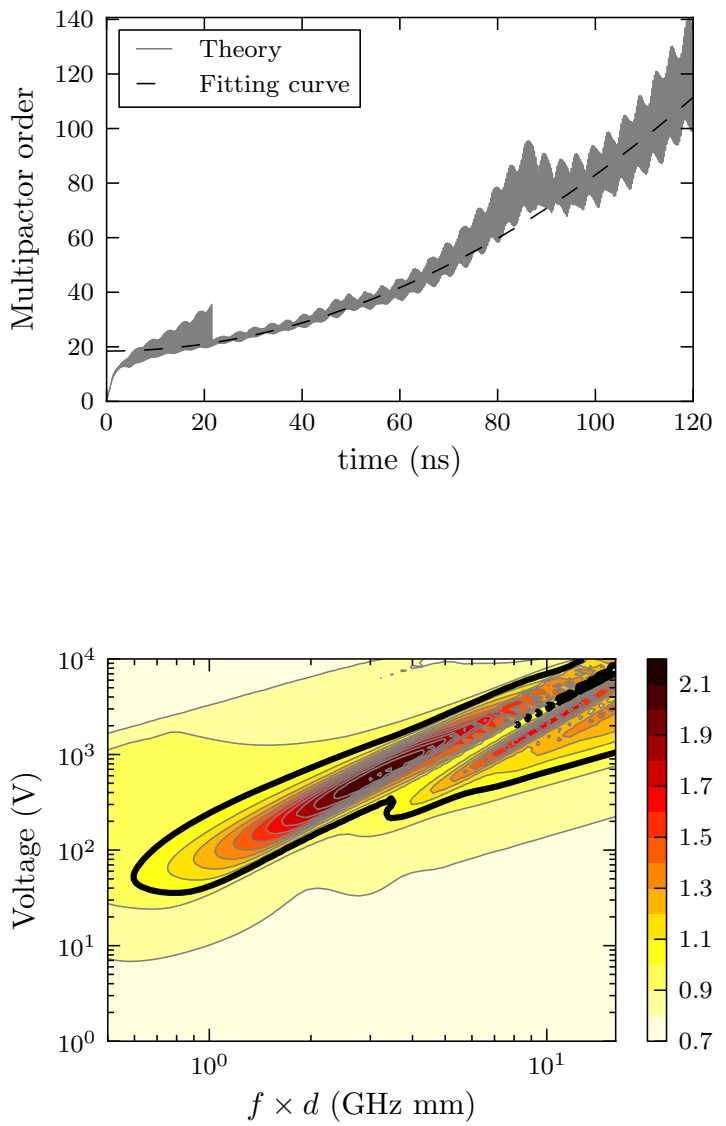

Figure 4.40.

Fitting of multipactor order. Frequency $=3.82$ GHz. $\quad d=1.3 \mathrm{~mm}$, silver plating and $V=$ $50 \mathrm{~V}\left(V_{s c}\right.$ around $\left.361 \mathrm{~V}\right)$. The result is $\alpha=6.5 \times$ $10^{15} s^{-2}$ and $\beta=18.43$.

Figure 4.41.

Multipactor map for $\tilde{\sigma}(V)$ for ECSS silver [EST03]

by using the QS approximations (4.94), (4.95) and (4.96), (4.93) becomes

$$
\frac{d N(t)}{d t}=N(t)[\tilde{\sigma}(V)-1] \frac{2 f_{m}}{\tilde{n}\left(V, t_{r}(t)\right)} .
$$

Note that $V \equiv V_{e}(t)$ is a function of $t$. The first-order ordinary differential equation given by (4.97) can be solved numerically with any standard method such as Runge-Kutta method. Its solution 
4.5. Prediction of Multipactor Breakdown for Multi-carrier Applications: The Quasi-stationary Method

Figure 4.42.

Multipactor for $\beta(V)$ for silver [EST03]
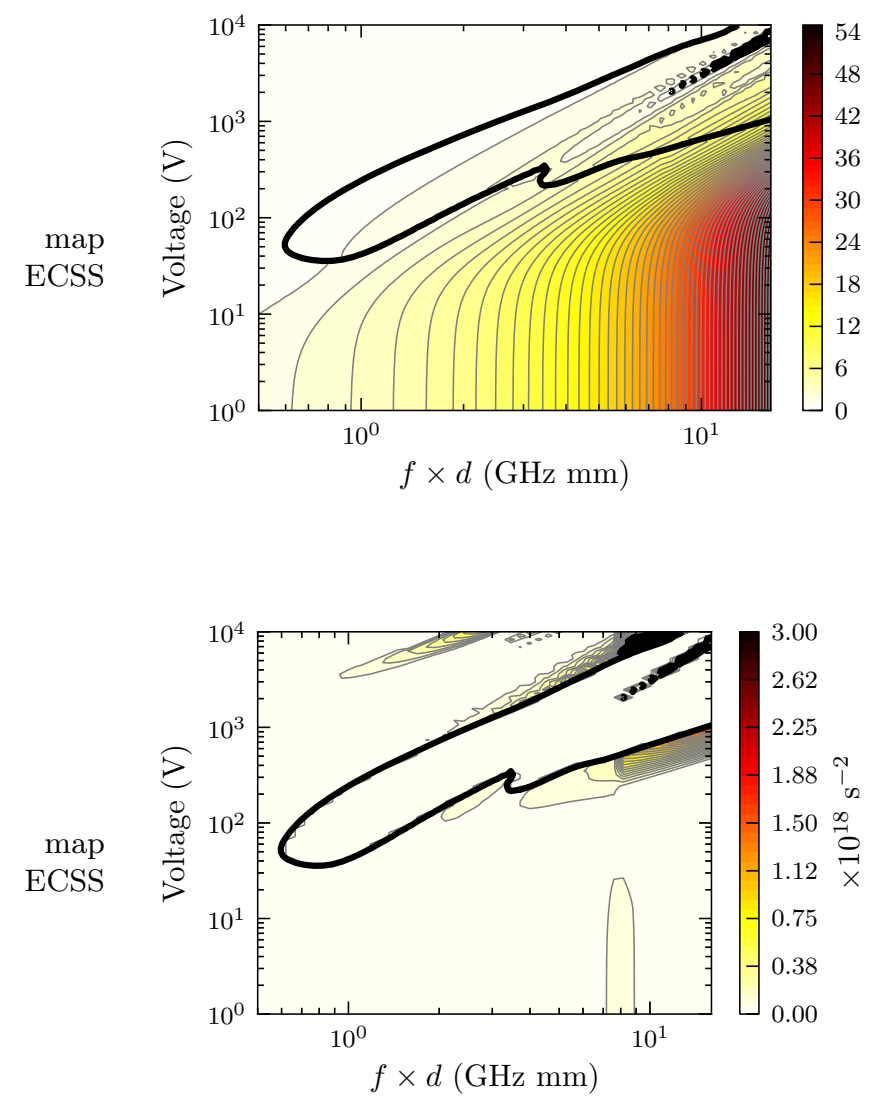

Figure 4.43.

Multipactor

for $\alpha(V)$ for ECSS silver [EST03].

provides the electron temporal evolution for a time varying amplitude $V$.

Finally, the criterion of multipactor is based on a long-term discharge where the electron multiplication $\Gamma(T)$, for an envelope of period $T$, must be greater than unity.

$$
\Gamma(T)=\frac{N(T)}{N(0)} \geq 1 .
$$

The QS model can compute $\Gamma(T)$ for any arbitrary multi-carrier envelope. 


\subsubsection{Procedure for threshold determination}

A specific example has been chosen to illustrate the procedure for the threshold determination using the QS model. The example is based on a 6-carrier signal centered at $3.82 \mathrm{GHz}$ with a frequency spacing of $\Delta f=100 \mathrm{MHz}$ and a gap of $d=1.31 \mathrm{~mm}$. The SEY parameters of the Vaughan's model [ $\mathrm{VMW}^{+} 05 \mathrm{a}$ ] have been chosen as $W_{1}=29$ $\mathrm{eV}, W_{\max }=399 \mathrm{eV}, \sigma_{\max }=2$ and $\sigma_{0}=0.5$, for the first cross-over energy, the energy of maximum SEY, the maximum SEY, and the SEY for low electron impact energies, respectively. Fig. 4.44 shows a diagram with the work-flow of this procedure.

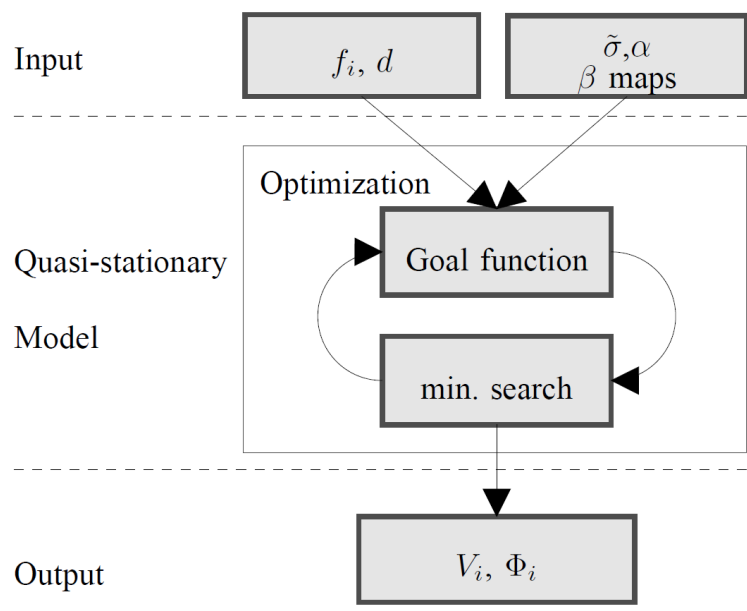

Figure 4.44.

Quasi-stationary model prediction flow chart.

For the specific mean frequency of the multi-carrier signal $\left(f_{m}\right)$ and sample gap size of the problem, the $f \times d$ of operation is derived, for which the SEY, $\alpha$ and $\beta$ curves versus signal amplitude are obtained. These curves can be interpolated from precomputed singlecarrier maps for the specific SEY parameters of the sample (such as those of Figs. 4.41-4.43), or can be ad-hoc computed using the singlecarrier non-stationary theory. Figs. 4.45 and 4.46 show the $\alpha$ and $\beta$ curves for this example. See that $\alpha=0$ above the breakdown level (located at $371 \mathrm{~V}$ ).

With this input data, the QS model is able to approximate the electron growth for any phase distribution and amplitude of the signal carriers. For example, in Fig. 4.47 the electron growth for a triangular 
4.5. Prediction of Multipactor Breakdown for Multi-carrier Applications: The Quasi-stationary Method

Figure 4.45.

Curve of SEY versus envelope amplitude for $f \times$ $d=5.002 \mathrm{GHz} \cdot \mathrm{mm}$.
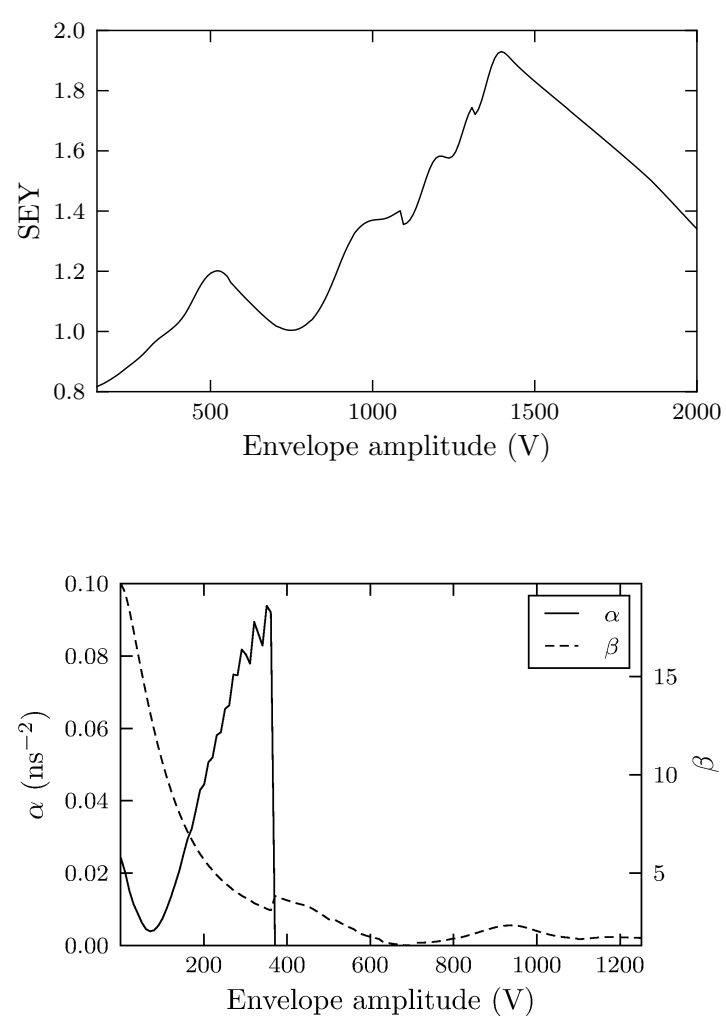

Figure 4.46.

Curve of $\alpha$ and $\beta$ versus envelope amplitude for $f \times d=5.002 \mathrm{GHz} \cdot \mathrm{mm}$.

phase scheme and three different values of the amplitude per carrier (equal for all carriers) is shown. These curves are computed with (4.97) and it is clear that there is electron accumulation $(\Gamma>1)$, and therefore multipactor discharge, for $V_{0}=142 \mathrm{~V}$, no discharge $(\Gamma<1)$ for $V_{0}=125 \mathrm{~V}$. The breakdown limit $(\Gamma=1)$ is obtained for $V_{0}=132 \mathrm{~V}$.

The QS prediction technique uses a global optimizer to search the combination of phases and amplitudes in order to minimize a goal function. Such a goal function must ensure that its minimum corresponds to the worst case. In this example the goal function is

$$
G=f(\Gamma)+V_{0},
$$

where equal amplitude of carriers, $V_{0}$, has been assumed (this method 

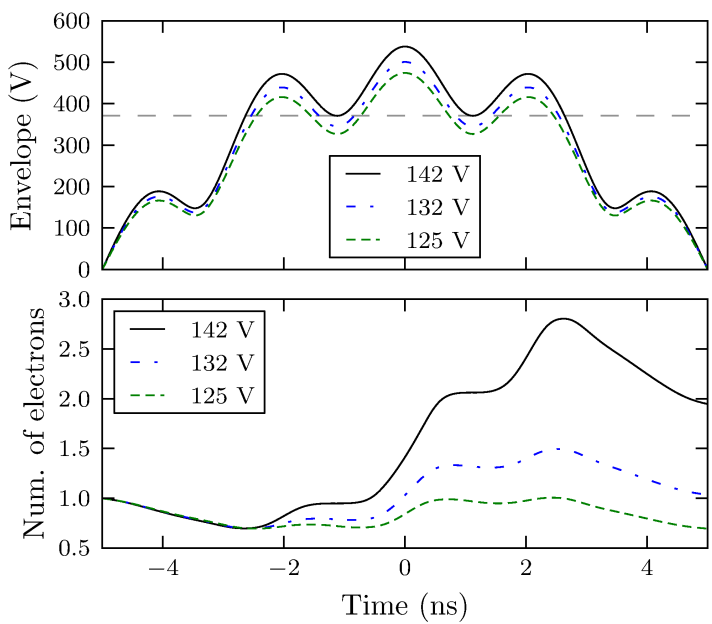

Figure 4.47.

Electron growth (bottom) according to QS model for different amplitude envelopes of a multi-carrier signal (top). The single-carrier threshold is marked with a horizontal dotted line.

would be also valid for unequal amplitudes). $\Gamma$ is given by (4.98), and

$$
f(\Gamma)=\left\{\begin{array}{ll}
w|\Gamma-1|, & \Gamma<1 \\
0, & \Gamma \geq 1
\end{array},\right.
$$

defines a constraint on the solution, penalizing regions where there is no multipactor $(\Gamma<1)$. No universal values can be provided for the weight $w$, which can be tuned to speed up the optimizer or to improve the accuracy of the solution.

The solution of the optimizer (minimizing $G$ ) is already the worst case for this signal, consisting of the combination of phases that ensures a multipactor discharge for the lowest carrier amplitude. However, keep in mind that the QS model is able to compute other breakdown levels than the lowest one, for any phase and amplitude distribution.

\subsubsection{Testing}

The objective of the test campaign is to provide meaningful data for different types of situations, such as different gap sizes, number of carriers and carrier phasing, with the aim of demonstrating the correct behavior of the new prediction tool. In order to do so, specific hardware has been manufactured. 
4.5. Prediction of Multipactor Breakdown for Multi-carrier Applications: The Quasi-stationary Method

Table 4.16. Ku-band frequency scheme.

\begin{tabular}{|c|c|c|c|c|c|c|}
\hline \multicolumn{3}{|c|}{ Channels } & \multicolumn{4}{|c|}{ Carrier combination } \\
\hline \multirow[t]{2}{*}{$\#$} & freq. & BW & conti & uous & non $\mathrm{c}$ & iguous \\
\hline & $(\mathrm{GHz})$ & $(\mathrm{MHz})$ & $6 \mathrm{ch}$. & $8 \mathrm{ch}$. & $6 \mathrm{ch}$. & $8 \mathrm{ch}$. \\
\hline 1 & 11.7440 & 34 & & & & $\mathrm{x}$ \\
\hline 2 & 11.8206 & 34 & & & $\mathrm{x}$ & $\mathrm{x}$ \\
\hline 3 & 11.8589 & 34 & & $\mathrm{x}$ & & \\
\hline 4 & 11.8972 & 34 & $\mathrm{x}$ & $\mathrm{x}$ & $\mathrm{x}$ & $\mathrm{x}$ \\
\hline 5 & 11.9355 & 34 & $x$ & $\mathrm{x}$ & & \\
\hline 6 & 11.9738 & 34 & $\mathrm{x}$ & $\mathrm{x}$ & $\mathrm{x}$ & $\mathrm{x}$ \\
\hline 7 & 12.0121 & 34 & $\mathrm{x}$ & $\mathrm{x}$ & & \\
\hline 8 & 12.0504 & 34 & $\mathrm{x}$ & $\mathrm{x}$ & $\mathrm{x}$ & $\mathrm{x}$ \\
\hline 9 & 12.0887 & 34 & $\mathrm{x}$ & $\mathrm{x}$ & & \\
\hline 10 & 12.1270 & 34 & & $\mathrm{x}$ & $\mathrm{x}$ & $\mathrm{x}$ \\
\hline 11 & 12.2036 & 72 & & & $\mathrm{x}$ & $\mathrm{x}$ \\
\hline 12 & 12.2802 & 72 & & & & $\mathrm{x}$ \\
\hline & $\begin{array}{l}f_{m}(\mathrm{GH} \\
\Delta f(\mathrm{Ml}\end{array}$ & & & & & \\
\hline
\end{tabular}

\subsubsection{Frequency plans and tests}

The frequency plan has been chosen to be in the Ku-band. In order to use different frequency schemes, 4 different signals have been tested: 6 carriers and 8 carriers with contiguous and non-contiguous schemes. A 15-channel Ku-band manifold output multiplexer has been selected for this purpose. The frequency plan of this multiplexer is given in Table 4.16.

8 power amplifiers were available, providing an equivalent peak power level of about $19000 \mathrm{~W}$.

\subsubsection{Sample Design}

A total number of $7 \mathrm{Ku}$-Band waveguide samples have been designed and manufactured with WR 75 interfaces (see Table 4.17).

The gaps of the samples have been selected in order to guarantee that the multipactor mode order is relatively low, and that no change 
Table 4.17. Samples for Ku-band tests.

\begin{tabular}{|c|c|c|c|c|c|c|}
\hline Type & $\begin{array}{l}\text { Gap } \\
(\mathrm{mm})\end{array}$ & $\begin{array}{l}V_{1 W} \\
(\mathrm{~V})\end{array}$ & $\begin{array}{l}\text { freq. } \\
\text { scheme }\end{array}$ & $\begin{array}{c}f_{m} \\
(\mathrm{GHz})\end{array}$ & $\begin{array}{l}f \times d \\
(\mathrm{GHz} \cdot \mathrm{mm})\end{array}$ & $n$ \\
\hline \multirow{6}{*}{$\mathrm{TF}$} & \multirow{2}{*}{0.14} & \multirow{2}{*}{3.89} & cont. & 11.99 & 1.68 & 1 \\
\hline & & & non cont. & 12.01 & 1.68 & 1 \\
\hline & \multirow{2}{*}{0.42} & \multirow{2}{*}{8.88} & cont. & 11.99 & 5.04 & 3 \\
\hline & & & non cont. & 12.01 & 5.05 & 3 \\
\hline & \multirow{2}{*}{0.64} & \multirow{2}{*}{10.38} & cont. & 11.99 & 7.67 & 5 \\
\hline & & & non cont. & 12.01 & 7.69 & 5 \\
\hline \multirow{6}{*}{ LP } & \multirow{2}{*}{0.14} & \multirow{2}{*}{6.35} & cont. & 11.99 & 1.68 & 1 \\
\hline & & & non cont. & 12.01 & 1.68 & 1 \\
\hline & \multirow{2}{*}{0.42} & \multirow{2}{*}{9.14} & cont. & 11.99 & 5.04 & 3 \\
\hline & & & non cont. & 12.01 & 5.05 & 3 \\
\hline & \multirow{2}{*}{0.64} & \multirow{2}{*}{11.5} & cont. & 11.99 & 7.67 & 5 \\
\hline & & & non cont. & 12.01 & 7.69 & 5 \\
\hline \multirow{2}{*}{$\mathrm{BP}$} & \multirow{2}{*}{1.31} & \multirow{2}{*}{32.1} & cont. & 11.99 & 15.71 & 7 \\
\hline & & & non cont. & 12.01 & 15.74 & 7 \\
\hline
\end{tabular}

of mode order will occur over the bandwidth of the sample.

The samples have been designed to be as simple as possible, and to avoid effects that may lead to a distortion of the results (e.g. no screws have been used for filter tuning and fringing field effects have been minimized).

Three different kinds of samples have been selected:

- Waveguide with reduced height and transformers on both ends (TF).

- Corrugated lowpass filter (LP).

- Narrow-band bandpass filter with inductive irises and reduced height (BP).

All samples have been built up in a two-shell configuration. The units are provided with silver plated surfaces. Fig. 4.48 shows the structure and electric field distribution for one example of each of 
4.5. Prediction of Multipactor Breakdown for Multi-carrier Applications: The Quasi-stationary Method

the three types of samples (other samples with different gap size are very similar). Fields have been computed with FEST3D [FES].

\section{Figure 4.48.}

Sample structure and electric field distribution for the three types of samples. From left to right: Transformer 0.14 $\mathrm{mm}$ gap, Low-pass filter $0.14 \mathrm{~mm}$ gap, Bandpass filter $1.31 \mathrm{~mm}$ gap. Reference grid lines separated $10 \mathrm{~mm}$ are included for dimensional reference. The magnitude of the maximum electric field is represented. Fields have been computed with FEST3D [FES].

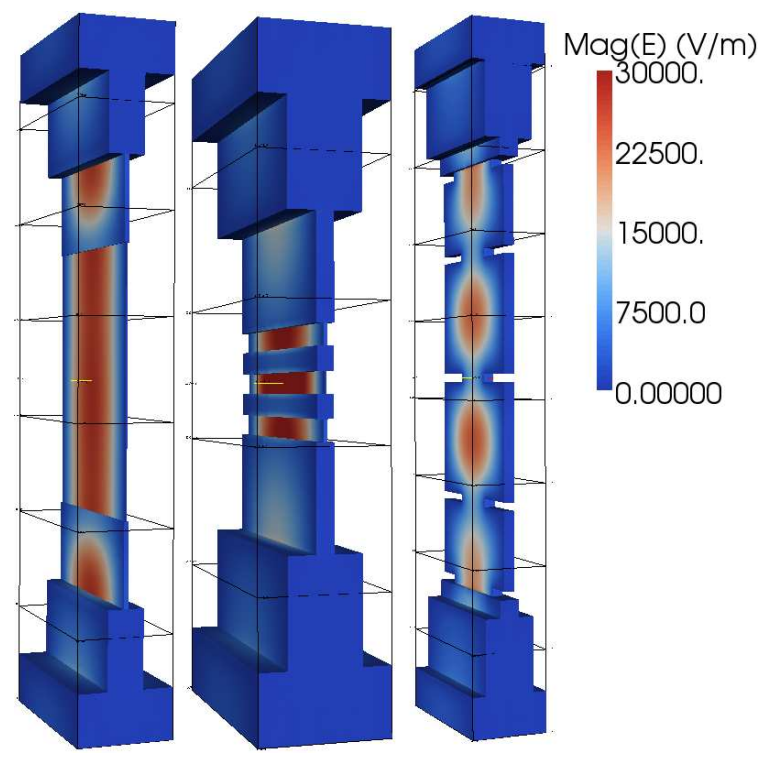

\subsubsection{Description of the test bed}

The schematic of the test set-up is shown in Fig. 4.49.

The tests have been performed at ambient temperature $\left(24^{\circ} \mathrm{C}\right)$. The chamber pressure was below $1 \mathrm{mPa}$, before starting the multipactor tests.

For the multi-carrier test signal generation, the power of 6 to 8 amplified channels have been combined with a multiplexer. For a steady multi-carrier signal, the phase relation between the single channels has been monitored and adjusted by a phase control unit (computer controlled unit).

Different techniques have been used to detect the occurrence of multipaction:

1. Input reflection nulling.

2. Near band noise. 


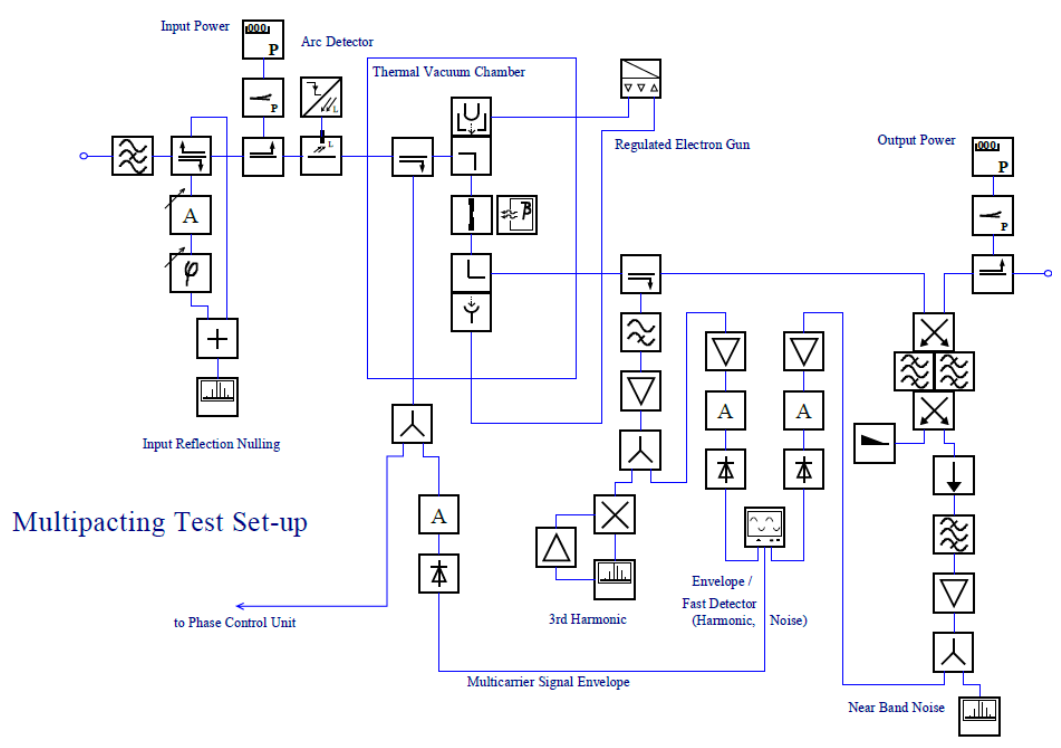

Figure 4.49. Schematic for Ku-band test set-up.

3. Fast diode detector for near band noise.

4. 3rd harmonic.

5. Fast diode detector for 3rd harmonic.

An electron gun and a remote controlled radioactive source have been used to provide a sufficient amount of free electrons to start the multipacting discharge.

A picture of the vaccum chamber and the test set-up for a $\mathrm{Ku}-$ band sample is shown in Fig. 4.50.

\subsubsection{SEY measurements}

In order to properly characterize the coating material and obtain good multipactor predictions, it was also necessary to measure the SEY of the different devices.

Since the dimensions of the devices were too large to fit inside the vacuum chamber of the SEY test, the measurements were done for 


\section{Figure 4.50.}

$\mathrm{Ku}$-band test set-up: Gap sample (a), radioactive source (b), electron gun (c) and coupler for detection of the phase and envelope (d) installed inside the thermal vacuum chamber.

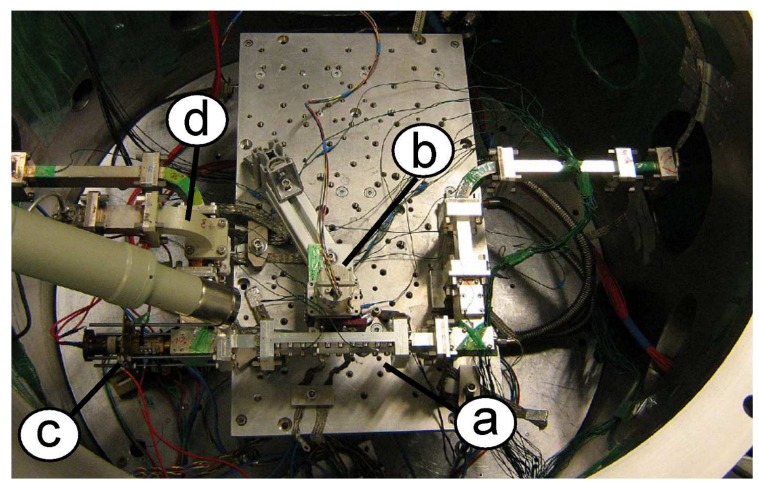

silver-plated aluminium alloy samples of $50 \times 20 \times 1 \mathrm{~mm}$, which were plated in the same bath used with each of the Ku-band manufactured devices.

The measured SEY parameters are given in Table 4.18. The SEY measurement is done over a spot of only $2 \mathrm{~mm}$ diameter and two measurements have been done for each sample. The manufacturing and plating process is identical for all of them, but significant differences can be appreciated. This is probably due to inhomogeneities in the surface which causes local SEY variations, thus implying a relative dependence of the results on the measurement point. Fig. 4.51 shows as an example the SEY curve measured for one of the samples compared with the Vaughan model used in this work.

Figure 4.51.

Comparison between measurements and Vaughan model for Transformer of $0.42 \mathrm{~mm}$ gap sample.

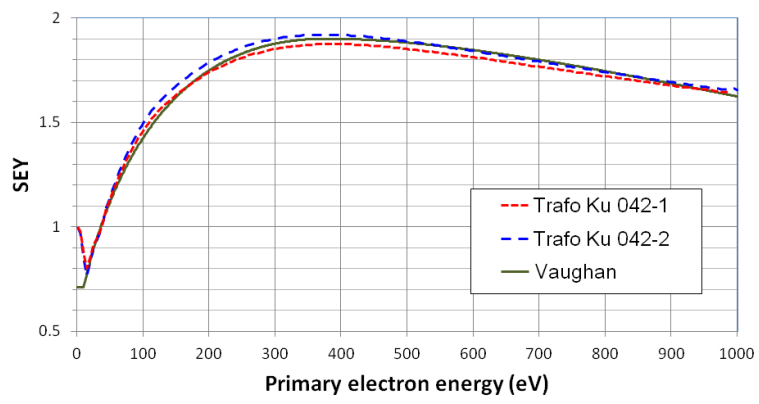




\begin{tabular}{cccccc} 
Type & $\begin{array}{c}\text { Gap } \\
(\mathrm{mm})\end{array}$ & $\begin{array}{c}W_{1} \\
(\mathrm{eV})\end{array}$ & $\begin{array}{c}W_{\max } \\
(\mathrm{eV})\end{array}$ & $\sigma_{\max }$ & $\sigma_{0}$ \\
\hline \hline \multirow{3}{*}{$\mathrm{TF}$} & 0.14 & 38 & 369 & 1.84 & 0.5 \\
\cline { 2 - 6 } & 0.42 & 36 & 378 & 1.9 & 0.5 \\
\cline { 2 - 6 } & 0.64 & 30 & 356 & 2.23 & 0.5 \\
\hline \hline \multirow{3}{*}{$\mathrm{LP}$} & 0.14 & 47 & 403 & 1.73 & 0.5 \\
\cline { 2 - 6 } & 0.42 & 32 & 370 & 2.1 & 0.5 \\
\cline { 2 - 6 } & 0.64 & 25 & 325 & 2.2 & 0.5 \\
\hline \hline $\mathrm{BP}$ & 1.3 & 25 & 309 & 2.2 & 0.5 \\
\hline \hline
\end{tabular}

Table 4.18.

SEY parameters for the Ku-band samples. The figures $W_{1}, W_{\max }, \sigma_{\max }$ and $\sigma_{0}$, stand for the first cross-over energy, the energy for maximum SEY, the maximum SEY and the SEY for low electron impact energies, respectively.

\subsubsection{Simulation software}

The 20GCR and the QS prediction techniques described in Sections 4.5.2.2 and 4.5.3, respectively, have been used to calculate the worst cases of the samples detailed in Section 4.5.4.2. The procedure for the threshold determination described in Section 4.5.3.2 has been followed. No extra margins have been applied to any of the prediction methods. The differential evolution algorithm [PSL05] has been employed for the QS optimization and for the 20GCR signal phase conforming.

All the worst case signals, predicted by the QS method, have been simulated with FEST3D [FES], a software for full-wave electromagnetic analysis and design of passive microwave circuits in waveguide technology, which also includes a RF high power module for multipactor analysis under multi-carrier operation.

\subsubsection{Predictions and test results}

\subsubsection{Error definition}

In our case, the output of the prediction, for a certain multi-carrier signal, is the couple consisting of phase distribution and power per carrier. The purpose of the prediction is to find the worst case, i.e. the combination of phases with the lowest breakdown power. We define:

- Local error: It is defined as the difference between the simulated 
4.5. Prediction of Multipactor Breakdown for Multi-carrier Applications: The Quasi-stationary Method

breakdown power and the experimental one measured when applying the computed phase distribution. This error gives an idea of the accuracy of the predictor to correlate the carrier phases and the breakdown power.

- Global error: The global prediction error is defined as the difference between the simulated breakdown power and the lowest breakdown power of all the tests made with the available phase conditions. This error measures the capability of predicting the lowest breakdown power.

A predictor that yields a low local error and a high global error, means that it is good to predict the breakdown power of a particular phase distribution, but the optimization of the phases fails to find the lowest breakdown power.

A low global error and high local error means that the predictor apparently is able to find the lowest breakdown power, but the worst case phase distribution does not correspond to the predicted one. This may happen for some specific cases, but it is unlikely that such a predictor is able to find the lowest breakdown power in a general case.

Finally, a good predictor is one that keeps both errors low.

\subsubsection{Phase configurations}

Three phase configurations have been used for each test, belonging to the following list:

- In phase (IP): All phases are set to zero.

- QS model (QS): The phases are optimized using the QS method of Section 4.5.3.2.

- 20-gap-crossing rule (20g): The phases are optimized to comply with the 20-gap-crossing rule worst case phasing, following Section 4.5.2.2.

The IP phasing has been tested in all samples. In the case that any of the predictions (QS or 20GCR) were equal to the IP phasing, such phase configuration was changed to "free running" phases (FR). 
In this situation the local oscillators of the amplifiers are left unlocked to the common reference and, hence, their relative phases change randomly. According to the authors' experience, this kind of test usually yields the lowest breakdown power. Therefore, even if it does not give any information on the envelope or phases, it may be a good reference for computing the global error (see Section 4.5.6.1).

Sometimes, not only one but both prediction methods, QS and 20GCR, yielded a solution similar to the IP case. In this case the two phase configurations were changed to FR condition and a nonoptimum QS prediction (QSn), i.e., another phase configuration which is not the worst case but for which the QS model can compute a breakdown prediction. This is useful in order to check the QS model prediction local error.

\subsubsection{RF breakdown prediction and measurements}

Table 4.19 shows the predicted worst cases for the two different analytical methods. Labels "c" and "n" stand for contiguous and noncontiguous frequency schemes. Breakdown levels are given in power per carrier. For the $20 \mathrm{GCR}$ prediction, a phase optimization has been run to make the envelope match with the desired number of gap crossings. The QS method has been used in order to find the phases that ensures a multipactor discharge with the minimum input power (worst case). The QS method does not employ the number of gap crossings in the optimization procedure. It gives directly the phase distribution. However, the number of gap crossings has been also computed and included in order to compare with the 20GCR.

The predicted breakdown power may be better seen in Fig. 4.52, where the boundaries for the breakdown powers are also plotted according to the $K$ and $K^{2}$ rules, $P_{s c} / K$ and $P_{s c} / K^{2}$, respectively, where $K$ is the number of carriers and $P_{s c}$ is the single carrier breakdown power determined by the single-carrier nonstationary theory of $\left[\mathrm{AVG}^{+} 10\right]$. FEST3D simulations are also included and labeled with "Num" tag.

As it can be seen, the predicted breakdown power levels computed by the 20GCR are significantly lower (around $3 \mathrm{~dB}$ ) than the QS method, which is closer to the $K$ power curve. 
4.5. Prediction of Multipactor Breakdown for Multi-carrier Applications: The Quasi-stationary Method

Table 4.19. Worst case prediction for Ku-band samples. Breakdown levels are given in power per carrier.

\begin{tabular}{|c|c|c|c|c|c|c|c|}
\hline \multirow[t]{2}{*}{ Type } & \multirow{2}{*}{$\begin{array}{l}\text { Gap } \\
(\mathrm{mm})\end{array}$} & \multirow[t]{2}{*}{ Sig. } & \multicolumn{2}{|c|}{ 20GCR } & \multicolumn{2}{|c|}{ QS } & \multirow{2}{*}{$\begin{array}{c}\text { FEST3D } \\
(\mathrm{W}) \\
\end{array}$} \\
\hline & & & $(\mathrm{W})$ & $n_{g c}$ & $(\mathrm{~W})$ & $n_{g c}$ & \\
\hline \multirow{12}{*}{$\mathrm{TF}$} & \multirow{4}{*}{0.14} & $6 c$ & 28.3 & 20 & 42.7 & 75 & 47.5 \\
\hline & & $6 n$ & 29.2 & 20 & 53.0 & 44 & 52.5 \\
\hline & & $8 \mathrm{c}$ & 16.0 & 20 & 36.7 & 75 & 29.38 \\
\hline & & $8 n$ & 16.9 & 20 & 36.3 & 38 & 32.5 \\
\hline & \multirow{4}{*}{0.42} & $6 \mathrm{c}$ & 65.7 & 20 & 178.3 & 47 & 580 \\
\hline & & $6 \mathrm{n}$ & 82.2 & 20 & 231.2 & 37 & 460 \\
\hline & & $8 c$ & 39.5 & 20 & 159.3 & 52 & 330 \\
\hline & & $8 \mathrm{n}$ & 57.2 & 20 & 174.8 & 38 & 215 \\
\hline & \multirow{4}{*}{0.64} & $6 c$ & 106.8 & 20 & 296.2 & 25 & 390 \\
\hline & & $6 n$ & 176.5 & 20 & 300.6 & 13 & 400 \\
\hline & & $8 c$ & 70.7 & 20 & 181.4 & 19 & 240 \\
\hline & & $8 \mathrm{n}$ & 131.7 & 20 & 183.0 & 10 & 255 \\
\hline \multirow{12}{*}{$\mathrm{LP}$} & \multirow{4}{*}{0.14} & $6 \mathrm{c}$ & 10.6 & 20 & 19.3 & 18 & 23.13 \\
\hline & & $6 n$ & 11.0 & 20 & 27.2 & 57 & 61.25 \\
\hline & & $8 \mathrm{c}$ & 6.0 & 20 & 18.0 & 107 & 20 \\
\hline & & $8 \mathrm{n}$ & 6.4 & 20 & 19.9 & 60 & 67.5 \\
\hline & \multirow{4}{*}{0.42} & $6 c$ & 56.2 & 20 & 154.0 & 38 & 480 \\
\hline & & $6 \mathrm{n}$ & 70.2 & 20 & 173.4 & 27 & 285 \\
\hline & & $8 c$ & 33.8 & 20 & 43.4 & 37 & 300 \\
\hline & & $8 \mathrm{n}$ & 48.8 & 20 & 137.8 & 31 & 310 \\
\hline & \multirow{4}{*}{0.64} & $6 c$ & 67.0 & 20 & 201.9 & 26 & 340 \\
\hline & & $6 n$ & 110.7 & 20 & 202.6 & 14 & 355 \\
\hline & & $8 c$ & 42.6 & 20 & 128.1 & 20 & 235 \\
\hline & & $8 \mathrm{n}$ & 82.6 & 20 & 128.4 & 10 & 245 \\
\hline \multirow{4}{*}{$\mathrm{BP}$} & \multirow{4}{*}{1.31} & $6 \mathrm{cc}$ & 66.4 & 20 & 96.7 & 211 & 220 \\
\hline & & $6 \mathrm{n}$ & 124.5 & 20 & 95.6 & 6 & 235 \\
\hline & & $8 \mathrm{c}$ & 48.9 & 20 & 132.1 & 14 & 162.5 \\
\hline & & $8 \mathrm{n}$ & 92.2 & 20 & 66.1 & 7 & 175 \\
\hline
\end{tabular}




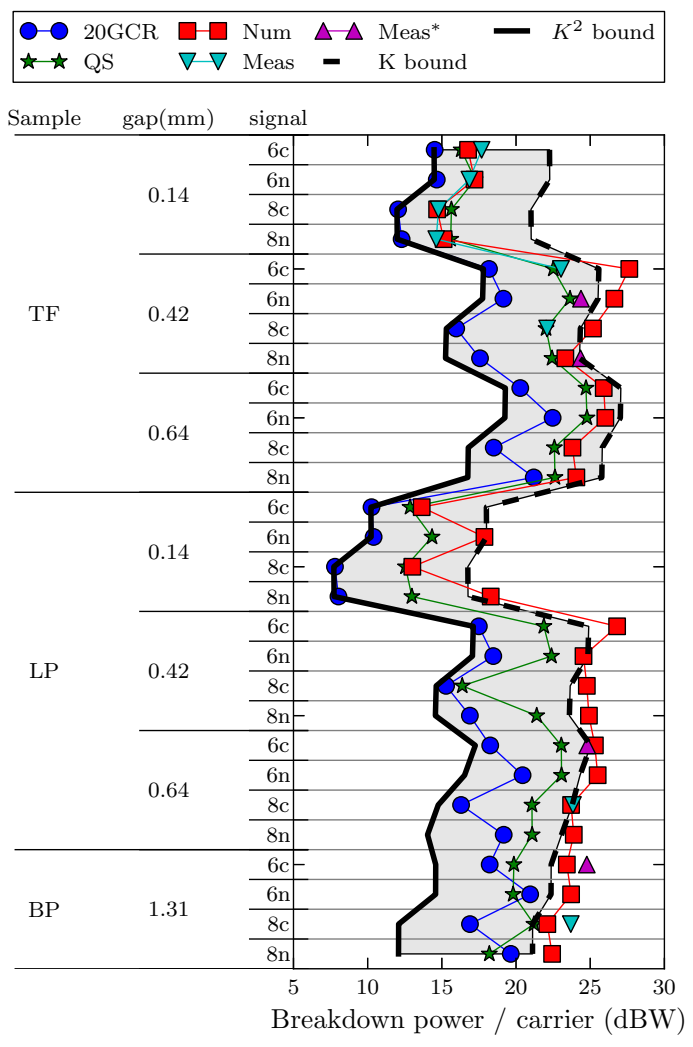

Figure 4.52.

Summary of breakdown threshold predictions and test results for $\mathrm{Ku}-$ band samples. FEST3D simulations are labeled with "Num" tag. Labels "c" and "n" stand for contiguous and non-contiguous frequency schemes. Breakdown levels are given in power per carrier. The minimum breakdown power of all tested phase configurations has been plotted with label "Meas". The maximum applied power for the cases where no multipactor has been observed has been also included with label "Meas"".

The number of gap crossings is shown in Fig. 4.53. Whereas the number of gap crossings is obviously fixed to 20 for the $20 \mathrm{GCR}$, the QS method yields a number of gap crossings that is different for each particular sample and signal having a great variation between cases. This is because each sample has a different SEY curve and work at a different $f \times d$ product, and it is also expected that a different number of gap crossings is needed to produce a discharge.

The number of gap crossings predicted by the QS method start at a high value (100-200) for the $0.14 \mathrm{~mm}$ gap samples, and follow a decreasing trend as the gap increases, finishing below 20 for the largest gaps, i.e. for $0.64 \mathrm{~mm}$ and $1.31 \mathrm{~mm}$.

Please note that a number of gap crossings of 100-200 for some 
Figure 4.53.

Summary of the number of gap crossings of predictions for $\mathrm{Ku}$-band samples.
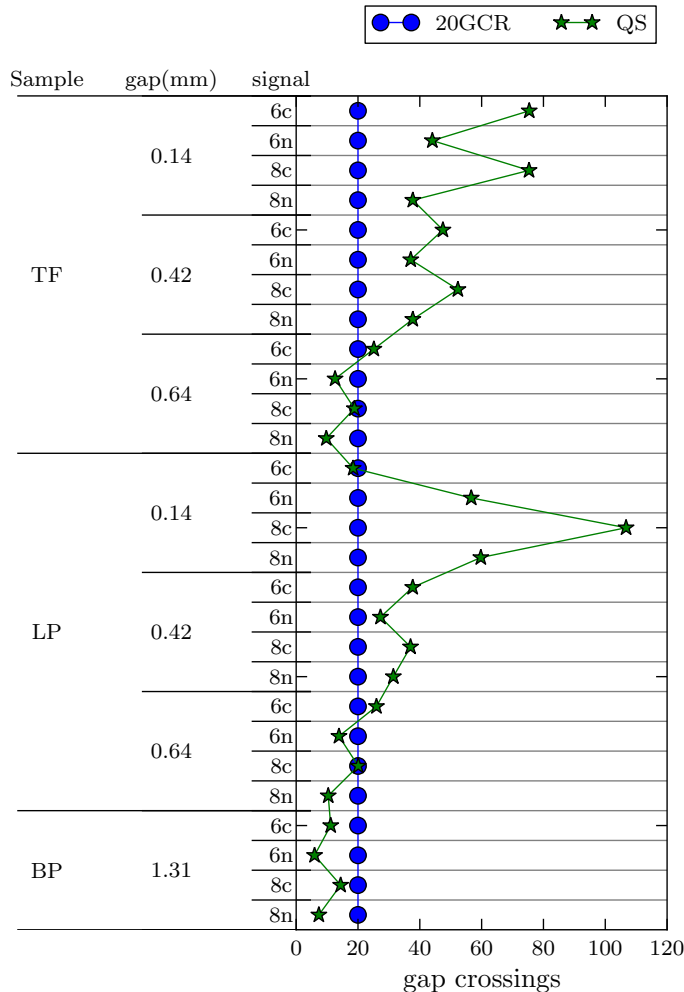

of the results corresponding to the $0.14 \mathrm{~mm}$ gap samples, is not as excessive as it may appear. The frequency spacing yields a period of the envelope of $26.11 \mathrm{~ns}$ and $13.05 \mathrm{~ns}$ for the contiguous and noncontiguous channel frequency schemes, respectively. For a center frequency of around $12 \mathrm{GHz}$, this implies a number of cycles of 627 and 313 , in one period of the envelope, for each of the two frequency schemes. Therefore, the $0.14 \mathrm{~mm}$ gap samples, working at a nominal multipactor order of $n=1$, have a total of 627 and 313 gap crossings within a period of the envelope. In this case, a number of gap crossings of 100-200 implies only a $T_{O N}$ of around a $16-32 \%$ and $32-64 \%$ of the total period $T$, respectively, for the two frequency schemes. Contrarily, 20 gap crossings yield a $T_{O N}$ of only $3-6 \%$ of the total envelope 
(approximately), which does in fact seem unrealistically low.

On the other hand, the $0.64 \mathrm{~mm}$ and $1.31 \mathrm{~mm}$ gap samples work at a nominal multipactor order of 5 and 7 , respectively. In this case, a number of gap crossings of 10 , for instance, implies around a $8-22 \%$ of the total envelope.

Table 4.20 and Fig. 4.52 show the experimental results for some of the samples and frequency schemes of the project. Breakdown levels are given in power per carrier. Two measurements have been carried out for every phase condition. Numerical results computed with FEST3D (marked as F3 in the table) have been also included. There is missing data for some of the samples, either because they have not been tested yet or because no multipactor has been detected up to the maximum available power (around $300 \mathrm{~W}$ per channel) in the set up (marked as "ND" in the table). More testing is envisaged in the future in order to have more comparison data. In Fig. 4.52, the minimum breakdown power of all tested phase configurations has been plotted with label "Meas" (corresponding to the definition of global error of Section 4.5.6.1). The maximum applied power for the cases where no multipactor has been observed has been also included with label "Meas".

It is worth noting that the experimental results are much closer to the QS predictions and to the FEST3D calculations than to the 20GCR predictions. The latter are in all cases much lower than the experimental results (around 3dB). As stated before, no extra margins have been applied to the predictions. This reveals that in fact the 20GCR seems to be very conservative, at least in the cases tested in this work.

Especially for large gaps, some FEST3D simulations yield a prediction above the theoretical $K$ rule bound. This in principle may seem unrealistic, but remember that both the $K^{2}$ and $K$ bounds are computed assuming parallel-plates, which is indeed the worst case. In real structures, the effect of the non-homogeneous fields, finite geometry and high order modes contribute to increase the breakdown power with respect to the parallel-plate approximation $\left[\mathrm{SRA}^{+}\right.$07]. FEST3D takes into account all the previous effects, and that is the reason why some of the predictions for large gaps lie above the theoretical upper bound. In fact, this may be also the reason why, in the laboratory, some of the samples did not exhibit a multipactor discharge up to 
4.5. Prediction of Multipactor Breakdown for Multi-carrier Applications: The Quasi-stationary Method

Table 4.20. Experimental results for the Ku-band samples. Breakdown levels are given in power per carrier.

\begin{tabular}{|c|c|c|c|c|c|c|c|c|c|c|c|c|c|c|}
\hline \multicolumn{2}{|c|}{$\begin{array}{l}\text { Type Gap } \\
(\mathrm{mm})\end{array}$} & $\mathrm{Sg}$. & $\mathrm{Cd}$. & $\Phi_{1}$ & $\Phi_{2}$ & $\Phi_{3}$ & \multicolumn{2}{|c|}{$\Phi_{4} \underset{(\operatorname{deg})}{\Phi_{5}}$} & $\Phi_{6}$ & $\Phi_{7}$ & $\Phi_{8}$ & $\begin{array}{l}\text { F3 } \\
\text { (W) }\end{array}$ & $\begin{array}{l}\text { M1 } \\
\text { (W) }\end{array}$ & $\begin{array}{l}\text { M2 } \\
(\mathrm{W})\end{array}$ \\
\hline \multirow{24}{*}{$\mathrm{TF}$} & \multirow{12}{*}{0.14} & & IP & $x$ & 0 & 0 & 0 & 0 & 0 & 0 & $x$ & 47.5 & $\overline{59.6}$ & $\overline{\overline{58.6}}$ \\
\hline & & $6 c$ & QSn & $\mathrm{x}$ & 0 & 0 & 180 & 180 & 0 & 0 & $\mathrm{x}$ & 170 & 78 & 73 \\
\hline & & & FR & - & - & - & - & - & - & - & - & - & 55.5 & 60 \\
\hline & & & IP & $x$ & 0 & 0 & 0 & 0 & 0 & 0 & $x$ & 52.5 & 53.5 & 51.8 \\
\hline & & $6 n$ & QSn & $\mathrm{x}$ & 0 & 0 & 180 & 180 & 0 & 0 & $\mathrm{x}$ & 78.8 & 89.5 & 89.5 \\
\hline & & & $\mathrm{FR}$ & - & - & - & - & - & - & - & - & - & 49 & 48.5 \\
\hline & & & IP & 0 & 0 & 0 & 0 & 0 & 0 & 0 & 0 & 29.4 & 30 & 30 \\
\hline & & $8 c$ & QSn & 0 & 350 & 331 & 299 & 299 & 331 & 350 & 0 & 39.4 & 61.5 & 59.5 \\
\hline & & & FR & - & - & - & - & - & - & - & - & - & 32 & 32 \\
\hline & & & IP & 0 & 0 & 0 & 0 & 0 & 0 & 0 & 0 & 32.5 & 29 & 29 \\
\hline & & $8 n$ & QSn & 0 & 180 & 180 & 0 & 0 & 180 & 180 & 0 & 48.1 & 47.5 & 47.5 \\
\hline & & & FR & - & - & - & - & - & - & - & - & - & 30.5 & 29.7 \\
\hline & \multirow{12}{*}{0.42} & & IP & $x$ & 0 & 0 & 0 & 0 & 0 & 0 & $x$ & 580 & ND & $\mathrm{ND}$ \\
\hline & & $6 c$ & QS & $\mathrm{x}$ & 0 & 180 & 0 & 0 & 180 & 0 & $\mathrm{x}$ & 700 & ND & ND \\
\hline & & & $\mathrm{FR}$ & - & - & - & - & - & - & - & - & - & 205 & 201 \\
\hline & & & IP & $\mathrm{x}$ & 0 & 0 & 0 & 0 & 0 & 0 & $\mathrm{x}$ & 500 & ND & ND \\
\hline & & $6 n$ & QS & $\mathrm{x}$ & 0 & 119 & 14 & 14 & 119 & 0 & $x$ & 860 & ND & ND \\
\hline & & & $20 \mathrm{~g}$ & $\mathrm{x}$ & 0 & 252 & 255 & 255 & 252 & 0 & $\mathrm{x}$ & 460 & ND & ND \\
\hline & & & IP & 0 & 0 & 0 & 0 & 0 & 0 & 0 & 0 & 330 & ND & $\mathrm{ND}$ \\
\hline & & $8 c$ & QS & 0 & 107 & 275 & 109 & 109 & 275 & 107 & 0 & 640 & 238 & 238 \\
\hline & & & FR & - & - & - & - & - & - & - & - & - & 164 & 162 \\
\hline & & & IP & 0 & 0 & 0 & 0 & 0 & 0 & 0 & 0 & 315 & ND & $\overline{\mathrm{ND}}$ \\
\hline & & $8 n$ & $20 \mathrm{~g}$ & 0 & 127 & 133 & 132 & 132 & 133 & 127 & 0 & 490 & ND & ND \\
\hline & & & QS & 0 & 109 & 256 & 56 & 56 & 256 & 109 & 0 & 215 & ND & ND \\
\hline \multirow{13}{*}{ LP } & \multirow{13}{*}{0.64} & & IP & $\mathrm{x}$ & $\overline{0}$ & $\overline{0}$ & 0 & 0 & $\overline{0}$ & $\overline{0}$ & $\bar{x}$ & 340 & - & - \\
\hline & & $6 c$ & QSn & $\mathrm{x}$ & 0 & 0 & 180 & 180 & 0 & 0 & $\mathrm{x}$ & 450 & - & - \\
\hline & & & FR & - & - & - & - & - & - & - & - & - & ND & ND \\
\hline & & & IP & $\mathrm{x}$ & 0 & 0 & 0 & 0 & 0 & 0 & $x$ & 355 & - & - \\
\hline & & $6 n$ & QSn & $\mathrm{x}$ & 0 & 0 & 180 & 180 & 0 & 0 & $\mathrm{x}$ & 470 & - & - \\
\hline & & & $20 \mathrm{~g}$ & $\mathrm{x}$ & 0 & 249 & 238 & 238 & 249 & 0 & $\mathrm{x}$ & 450 & - & - \\
\hline & & & IP & 0 & 0 & 0 & 0 & 0 & 0 & 0 & 0 & 235 & - & - \\
\hline & & & QSn & 0 & 180 & 180 & 0 & 0 & 180 & 180 & 0 & 315 & ND & ND \\
\hline & & $8 \mathrm{c}$ & $20 \mathrm{~g}$ & 0 & 238 & 237 & 238 & 238 & 237 & 238 & 0 & 355 & - & - \\
\hline & & & $\mathrm{FR}$ & - & - & - & - & - & - & - & - & - & 243 & 242 \\
\hline & & & $\mathrm{IP}$ & 0 & 0 & 0 & 0 & 0 & 0 & 0 & 0 & 245 & - & - \\
\hline & & $8 n$ & QSn & 0 & 180 & 180 & 0 & 0 & 180 & 180 & 0 & 320 & - & - \\
\hline & & & $20 \mathrm{~g}$ & 0 & 69 & 127 & 142 & 142 & 127 & 69 & 0 & 375 & - & - \\
\hline \multirow{14}{*}{ BP } & \multirow{14}{*}{1.31} & & $\overline{\mathrm{IP}}$ & $\bar{x}$ & $\overline{0}$ & $\overline{0}$ & $\overline{0}$ & $\overline{0}$ & $\overline{0}$ & $\overline{0}$ & $x$ & 220 & $\overline{-1}$ & $\overline{-}-$ \\
\hline & & $\rho$ & QSn & $\mathrm{x}$ & 0 & 0 & 180 & 180 & 0 & 0 & $\mathrm{x}$ & 230 & - & - \\
\hline & & $6 \mathrm{c}$ & $20 \mathrm{~g}$ & $\mathrm{x}$ & 0 & 105 & 123 & 123 & 105 & 0 & $\mathrm{x}$ & 225 & - & - \\
\hline & & & $\mathrm{FR}$ & - & - & - & - & - & - & - & - & - & ND & $\mathrm{ND}$ \\
\hline & & & IP & $\mathrm{x}$ & 0 & 0 & 0 & 0 & 0 & 0 & $\mathrm{x}$ & 275 & - & - \\
\hline & & $6 \mathrm{n}$ & QSn & $\mathrm{x}$ & 0 & 0 & 180 & 180 & 0 & 0 & $\mathrm{x}$ & 245 & - & - \\
\hline & & & $20 \mathrm{~g}$ & $\mathrm{x}$ & 0 & 62 & 136 & 136 & 62 & 0 & $\mathrm{x}$ & 235 & - & - \\
\hline & & & IP & $\mathrm{x}$ & 0 & 0 & 0 & 0 & 0 & 0 & $x$ & 190 & - & - \\
\hline & & 8 & QSn & 0 & 12 & 35 & 67 & 67 & 35 & 12 & 0 & 150 & 259 & 253 \\
\hline & & $8 \mathrm{c}$ & $20 \mathrm{~g}$ & 0 & 56 & 125 & 141 & 141 & 125 & 56 & 0 & 163 & - & - \\
\hline & & & $\mathrm{FR}$ & - & - & - & - & - & - & - & - & - & 233 & 234 \\
\hline & & & IP & 0 & 0 & 0 & 0 & 0 & 0 & 0 & 0 & 203 & - & \\
\hline & & $8 n$ & QSn & 0 & 180 & 0 & 180 & 180 & 0 & 180 & 0 & 185 & - & - \\
\hline & & & $20 \mathrm{~g}$ & 0 & 88 & 253 & 307 & 307 & 253 & 88 & 0 & 175 & - & - \\
\hline
\end{tabular}




\begin{tabular}{cccc} 
& 20GCR & QS & FEST3D \\
\hline \hline Local error $(\mathrm{dB})$ & 2.68 & 1.2 & 1.42 \\
Global error $(\mathrm{dB})$ & 4.47 & 1.18 & - \\
\hline \hline
\end{tabular}

Table 4.21.

Average prediction error for the prediction methods on the $\mathrm{Ku}$-band samples

the maximum available power, even if the $K$ bound lay below such maximum power.

\subsubsection{Analysis of results}

The average prediction error of all samples is presented in Table 4.21. The local error of the FEST3D numerical tool is also included for comparison.

The analysis of the errors indicates that the 20GCR shows a considerable higher local and global error with respect to the QS method. The latter shows very good figures for both of them. This indicates that the QS method shows the best prediction performance and, again, that the $20 \mathrm{GCR}$ is very conservative (more than $4 \mathrm{~dB}$ ).

The local error of the FEST3D simulations show similar values than for the QS method. This is because the designed samples are waveguides with long irises in order to resemble the parallel plate case as much as possible. With other more complicated geometries involving fringing field effects, numerical full-wave EM solvers such as FEST3D are expected to give more realistic results.

\subsubsection{Conclusions}

It is clear from the experimental results that the new QS prediction technique offers better predictions than the 20GCR. This fact was expected since the former uses a more sophisticated theoretical background based on multipactor physics. Moreover, for the prediction the QS method considers the frequency scheme and the SEY curve of the coating material for each specific case, offering more accuracy and versatility with respect to the $20 \mathrm{GCR}$.

Besides being more precise, the predicted breakdown power levels of the QS technique are significantly higher than the ones provided by the 20GCR. This is an important factor for the industry because 
4.5. Prediction of Multipactor Breakdown for Multi-carrier Applications: The Quasi-stationary Method

this would allow increasing the operating power of the devices, thus reducing the designs constraints and increasing the margins.

Finally, the QS method may use pre-calculated multipactor maps, in order to predict the worst case without having to implement any multipactor theory or use any multipactor numerical software.

\section{Acknowledgment}

The authors would like to thank Isabel Montero and Luis Galan of the Consejo Superior de Investigaciones Cientificas (CSIC) and the Universidad Autonoma de Madrid (UAM), respectively, for conducting the SEY measurements reported in Section 4.5.4.4. Thanks to ESA/ESTEC for having funded this research activity through the Contract "RF Breakdown in Multicarrier Systems" (19918/06/NL/GLC), and to the Ministerio de Ciencia e Innovacion (Spain) for the support through the "Programa Torres Quevedo" PTQ06-2-0693. 


\section{Chapter 5}

\section{General Discussion}

The articles presented in section 4 constitute the core of the research done in this Thesis. Basically, these papers cover, on the one hand, the development of a new non-stationary theory for single and multicarrier signals, and, on the other hand, the proposal of a new prediction method for multipactor in multi-carrier signals: The Quasistationary method.

This chapter will attempt to give a conceptual overview of the above articles, highlighting the differences with other theories, with special emphasis on multi-carrier threshold prediction. The experimental results of $\left[\mathrm{AVG}^{+} 12\right]$ will be revisited and discussed in a more general framework.

\subsection{Single-carrier theories}

\subsubsection{Classical multipactor theory}

The classical theory of multipactor [GvE48, HW54, Vau88] covers the particular case of two-surface multipactor in single-carrier regime on infinite parallel plates. The classical theory assumes that the electrons are emitted with a fixed and deterministic initial velocity. The 
${ }^{1}$ In most cases, the fields will have local resemblance to those of a parallel plate geometry. This is true at least for rectangular waveguides as $\left[\mathrm{SRA}^{+} 07\right]$ demonstrates results may be extrapolated to real structures provided that the gap is small compared to the rest of the device dimensions $\left[\mathrm{SRA}^{+} 07\right]^{1}$. Basically, the classical theory analytically solves the electron equations of motion looking for resonance and electron growth conditions. Fig. 5.1 shows the problem geometry and notation.

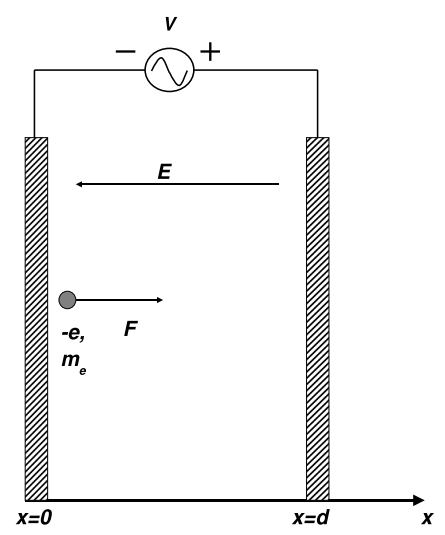

Figure 5.1.

Infinite parallel plate problem geometry and notation. Note that since the electric field and the electron charge are negative, the force on the electron is positive.

The equations of motion of an electron of charge $-e$ and mass at rest $m_{e}$ between two infinite parallel plates located in $x=0$ and $x=d$ are already given in $\left[\mathrm{AVG}^{+} 10\right]$ (Section 4.3 ), and are repeated here for completeness. For an RF electric field of amplitude $E_{0}$ and frequency $f$ (angular frequency $\omega=2 \pi f$ ), the acceleration exerted on the electron is

$$
m_{e} \ddot{x}=e E_{0} \sin (\omega t),
$$

where the RF voltage $V_{0}$ can be expressed as $V_{0}=E_{0} d$.

The initial conditions at starting time $t=t_{s}$ are

$$
\left.x\right|_{t=t_{s}}=0,\left.\quad \dot{x}\right|_{t=t_{s}}=v_{0},
$$

From (5.1) the rest of the equations of motion can be easily derived

$$
\begin{gathered}
\dot{x}=v_{0}+v_{\omega}\left[\cos \left(\omega t_{s}\right)-\cos (\omega t)\right] \\
x=\left[v_{0}+v_{\omega} \cos \left(\omega t_{s}\right)\right]\left(t-t_{s}\right)+\frac{v_{\omega}}{\omega}\left[\sin \left(\omega t_{s}\right)-\sin (\omega t)\right],
\end{gathered}
$$

where $v_{\omega}=e V_{o} /\left(m_{e} \omega d\right)$. 
The time $t_{i}$ at which the electron reaches the opposite plate $(x=$ $d$ ) is then the least root of

$$
d=\left[v_{0}+v_{\omega} \cos \left(\omega t_{s}\right)\right]\left(t_{i}-t_{s}\right)+\frac{v_{\omega}}{\omega}\left[\sin \left(\omega t_{s}\right)-\sin \left(\omega t_{i}\right)\right] .
$$

The conditions for a multipactor discharge to occur are:

1. In order to achieve resonance, the primary and secondary electron travel phases must be equal and have a value of an odd number of half-periods, i.e $n \pi$, where $n=1,3,5,7, \ldots$ is the multipactor modal order or multipactor mode.

2. The time or phase at which the electrons are released, must be within a certain range in order to be synchronized with the electric field (if the electron is released when the electric field pushes it in the wrong direction, against the originating wall, the electron will not depart).

3. The impacting energy (velocity) must be comprised between $W_{1}$ and $W_{2}$, which are the first and second cross-over energies of the material SEY, respectively.

From condition 1 and (5.1), (5.3) and (5.4), the expressions for the voltage amplitude, frequency, phase, and device gap can be derived. Conditions 2 and 3 define the ranges of such quantities that initiate a multipactor discharge.

There are two main families of classical theories, the constant- $k$ and the constant- $v$ theories that are detailed next.

\subsubsection{Constant- $k$ theory}

The constant- $k$ was originally proposed by the first work in Multipactor Theory made by Gill and von Engel [GvE48]. This theory fixes the ratio between electron impact velocity $v_{i}$ and release velocity $v_{0}$

$$
k=\frac{v_{i}}{v_{0}} .
$$

According to the first condition of multipactor, the electron starting at phase $\varphi_{s}=\omega t_{s}$ must arrive to the opposite plate, $x=d$, at 
a phase $\omega t_{i}=\varphi_{s}+n \pi$, being $n$ the multipactor order. Substituting (5.6) in (5.3) and taking into account that the RF voltage amplitude is $V_{0}=d E_{0}$, the expression of the impact velocity results

$$
v_{i}=\frac{k}{k-1} 2 v_{\omega} \cos \varphi_{s} .
$$

Performing the same operation with (5.4), the amplitude of the excitation voltage $V_{0}$ can be expressed in terms of the other parameters as

$$
V_{0}=\frac{(\omega d)^{2}}{\left(e / m_{e}\right) \Phi_{n}}
$$

and

$$
\Phi_{n}=\frac{k+1}{k-1} n \pi \cos \varphi_{s}+2 \sin \varphi_{s} .
$$

On the other hand, with respect to the second condition for multipactor, the maximum allowed departure phase $\varphi_{\max }$ to assure resonance conditions is the one that minimizes (5.8), thus maximizing (5.9):

$$
\varphi_{\max }=\tan ^{-1}\left(\frac{k-1}{k+1} \frac{2}{n \pi}\right) .
$$

Regarding the minimum starting phase, when the electron starts with negative phase, the electric field pushes it against the originating surface. Provided that the electron's initial velocity is higher than zero, the electron, after an initial backward movement may be able to reach the opposite side. The minimum phase $\varphi_{\min }$, allowing the electron to reach the opposite side is called the non-return point which does not have an analytical expression and must be solved numerically.

Finally, the third condition of multipactor imposes that the impact velocity must be comprised between the first and second crossover velocities of the surface material's SEY, $v_{1}$ and $v_{2}$, respectively (which relate to the cross-over energies $W_{1}$ and $W_{2}$ as $W=\frac{1}{2} \frac{m_{e}}{e} v^{2} \mathrm{eV}$ ).

Note that (5.8) is expressed as a function of the $f d$ product. Therefore, the combination of $f d, V_{0}$ and $\varphi_{s}$, that leads to a multipactor discharge can be represented in multipactor susceptibility regions, in a $V_{0}$ versus $f d$ plot. This kind of representation was first introduced by Hatch and William's work in [HW54] for the first 
multipactor mode $(n=1)$, and extended later in [HW58] for higher modes. Fig. 5.2 shows generic Hatch and William's curves for the first eleven modes. The boundaries A-D and B-C for the first mode correspond to the maximum and minimum allowed emission phase, whereas the boundaries A-B and C-D correspond to the impact energy and SEY equal to 1 condition. The region inside A-B-C-D is the multipactor region for the first mode. The same applies for the others. The contour lines represent the different values of the SEY inside the regions, which depend on the impact energy, given by (5.7), and are always higher than 1 . The Vaughan SEY model $\left[\mathrm{VMW}^{+} 05 \mathrm{a}\right]$ with ECSS silver [EST03] has been used.

\section{Figure 5.2.}

Hatch and William's chart for the first eleven modes with $k=5$ and first and second crossover energies $W_{1}=30 \mathrm{eV}$ and $W_{2}=2000 \mathrm{eV}$. The contour lines represent the different values of the SEY, for ECSS silver, according to the impact energies given by (5.7).

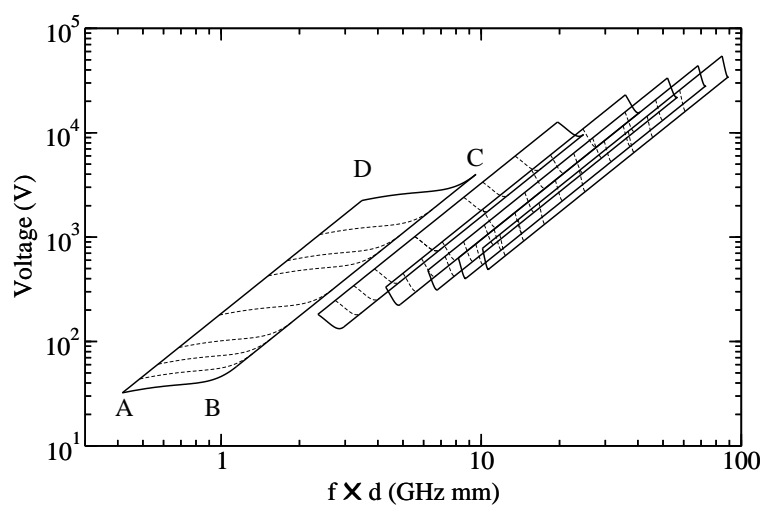

The Hatch and William's curves can fit the experimental multipactor results for any material by adjusting the parameters, $k, W_{1}$ and $W_{2}$. Hatch and William's curves have become the ESA / ESTEC standard multipactor susceptibility charts, since they show high flexibility to best fit the experimental data. Woode and Petit in [WJ89], performed a multipactor study, fitting the Hatch and William's curves to experimental data on rectangular waveguides and diverse material coatings, corroborating the latter conclusion. Fig. 5.3 shows the susceptibility curve for silver where the parameters $k$ and $W_{1}$ have been chosen to fit the experimental data.

The parameter fitting done by Woode and Petit to match experimental results is a very useful tool for predicting the breakdown threshold, but is physically incorrect, since it is unnatural to change 


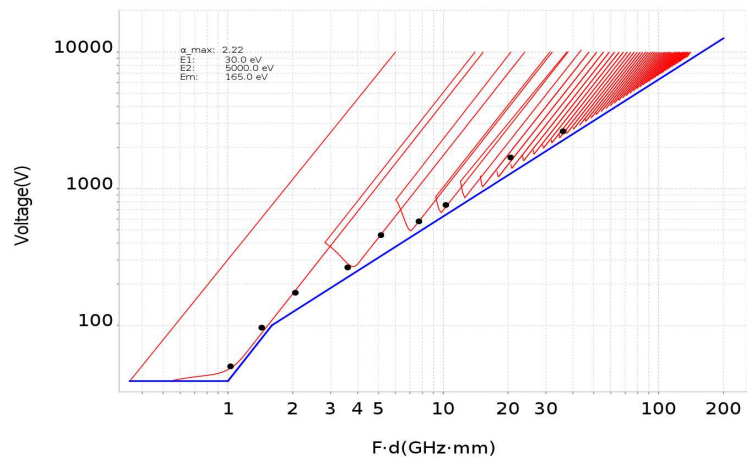

Figure 5.3.

Woode and Petit's fit of Hatch and William's chart for Silver, extracted from [ECS].

the SEY properties of the material at will at each $f d$ in order to match the results. In addition, a prediction tool that needs experimental data to work is a contradiction, for a prediction tool should allow for avoiding testing.

\subsubsection{Constant- $v$ theory}

In [Wac64, Vau88], the constant- $v$ theory is proposed as an alternative to the constant- $k$ theory, with the more realistic assumption of a monoenergetic nonzero initial velocity $v_{0}$. Using (5.3), (5.4) and the first multipactor condition, the expressions for the impact velocity and the rf peak voltage expressions can be derived in the same way as done in the constant- $k$ theory case, yielding

$$
v_{i}=2 v_{\omega} \cos \varphi_{s}+v_{0}
$$

and

$$
V_{0}=\frac{m_{e}}{e} \frac{\omega d\left(\omega d-n \pi v_{0}\right)}{n \pi \cos \varphi_{s}+2 \sin \varphi_{s}}
$$

respectively.

Note that expressions (5.11) and (5.12) for the constant- $v$ theory are very similar to (5.7) and (5.8) for the constant- $k$ theory. Indeed, both theories are equal for the special case of infinite $k$ or zero initial $v_{0}$.

The limiting starting phases are calculated from the second condition of multipactor in the same way as the constant- $k$ theory. The 
maximum $\varphi_{\max }$ is the one that minimizes (5.12) and is equal to

$$
\varphi_{\max }=\tan ^{-1}\left(\frac{2}{n \pi}\right) .
$$

And the minimum phase, $\varphi_{\min }$, corresponds to the non-return point which must be calculated numerically.

The multipactor regions can be also plotted in a $V_{0}$ versus $f d$ graph from (5.12). These are shown in Fig. 5.4.

\section{Figure 5.4.}

Constant- $v$ multipactor chart for the first eleven modes and energies $W_{0}=1.5 \mathrm{eV}, W_{1}=30$ $\mathrm{eV}$ and $W_{2}=2000$ $\mathrm{eV}$. The contour lines represent the different values of the SEY, for ECSS silver, according to the impact energies given by (5.11).

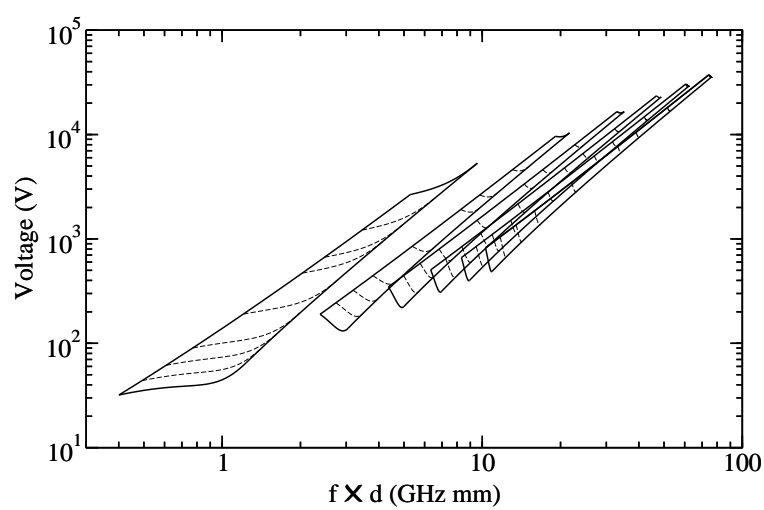

The constant- $v$ theory yields very similar regions compared with the constant- $k$ one. However, It can be observed that the lower and upper multipactor boundaries are not parallel as in the constant- $k$ theory but instead get narrower for increasing $f d$.

\subsubsection{Extended resonance conditions: hybrid modes}

In [Gil92], Gilardini shows theoretically the existence of a more general solution to the equation of electron motion (4.26) in order to achieve multipactor resonance in parallel plates. In contrast with the classical theory for the two-surfaces multipactor, in which the one-way transit time of one electron has to be an odd number of half-cycles, this solution implies that the two-way transit time of the electrons must be an integer number of field cycles, i.e. $\psi_{1}+\psi_{2}=2 q \pi$, where $\psi_{1}$ and $\psi_{2}$ are the primary and secondary adimensional transit times (expressed as $\psi=\omega t$ ) respectively and $q=1,2,3, \ldots$ This 
condition is more general than the classical one, since primary and secondary transit times can be different.

The new condition is equivalent to the classical one if $\psi_{1}=\psi_{2}=$ $n \pi$, where $n$ is an odd integer, and $\psi_{1}+\psi_{2}=2 n \pi=2 \pi, 6 \pi, 10 \pi, \ldots$ which is covered by the general multipactor condition for odd $q$. Therefore, the new modes are those with even $q$, where $\psi_{1}+\psi_{2}=$ $2 q \pi=4 \pi, 8 \pi, 12 \pi, \ldots$ For that reason, Gilardini calls them even modes. Solving the multipactor equation for even modes and representing them in a Hatch and Williams graph, they are found to have a very narrow multipactor zone located between the transition of classical odd modes, as shown in Fig. 5.5.

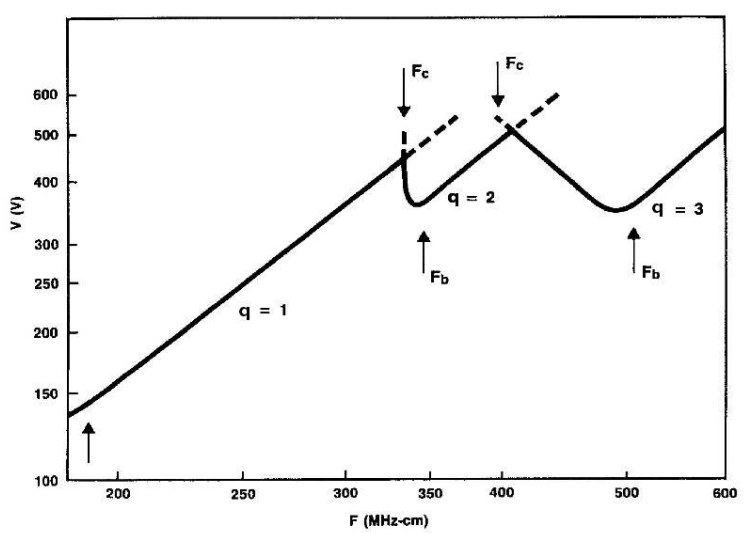

Figure 5.5.

Hatch and William's susceptibility curves with even hybrid modes. Only the lower boundary is drawn. Even hybrid modes (even q) are intercalated among the main modes (odd q) filling the discontinuities of the overall susceptibility region.

On the other hand Kryazhev proposes in $\left[\mathrm{KBS}^{+} 02\right]$ a hybrid resonance theory which is a generalization of the new modes discovered by Gilardini. Kryazhev extends this condition not only to the twoway transit time, but to $p$-way transit time, where $p$ is the order of the periodicity imposing only that the accumulated period has to be a multiple of $\pi$ or $2 \pi$ if $p$ is odd or even respectively. The hybrid modes have a very narrow multipactor region located in between main multipactor modes.

Recently, new Ping-Pong resonance modes have been proposed in [Kis12]. These special modes combine a single-surface interaction with a consecutive two-surface interaction. These resonances produce a multipactor discharge if the electrons produced in the two-surface interaction compensate the electron losses in the single-surface one. 
These modes are present at low values of $f d$ and high voltages and, therefore, produce a broadening of the multipactor regions of such areas.

\subsubsection{Phase focusing}

The second condition for multipactor stated by Gill and Von Engel implies that, when applying a certain RF field to the electrodes with certain field strength and frequency, there has to be at least one initial electron with the right starting phase in order to initiate multipactor. Gill and Von Engel justified that, since their experiment was carried out with low pressure gases, there was enough sources of free electrons in order to assure the existence of at least one electron in the whole range of valid phases [GvE48]. However, this seems not very reliable, even more when multipactor has been indeed observed in vacuum conditions where the source of free electrons is limited. The explanation comes from the stability of the electron motion or the phase focusing effect, which is the trend of the electrons with small errors in the departure phase to converge to the equilibrium one. The phase focusing effect allows for electrons with a wrong departure phase, but close enough to the right one, to participate in multipactor. Phase focusing is one of the stability mechanisms of multipactor and it is detailed in [Vau88, Gil95, She03].

The $\mathrm{G}$ factor is defined as $G \equiv \frac{\partial \varphi_{2}}{\partial \varphi_{1}}$, were $\varphi_{1}$ and $\varphi_{2}$ are the departure phase of the primary and secondary electrons, respectively. Thus, the condition for the electron motion to be linearly stable is [Vau88, Gi195]

$$
|G|<1 \text {. }
$$

In $\left[\mathrm{KBS}^{+} 02\right]$ a more relaxed condition of stability is presented, in which unstable regions may develop a discharge if the SEY is large enough to compensate the electron debunching. This simply means that

$$
|G|<\sigma
$$

where $\sigma$ is a particular SEY value.

Shemelin presents in [She03] a generalized theory of phase stability, or phase focusing, which justifies some of the discrepancies 
in the definition of the multipactor boundaries for each mode between theoretical Hatch and William's curves and experimental results. Shemelin establishes that, in order to achieve multipactor, the starting phase of electrons must either converge to an equilibrium value (classical phase focusing) or vary in time with a periodical structure, provided that the resonance condition and minimum impact electron velocity conditions are satisfied for both cases. Fig. 5.6 extracted from [She03] shows two different evolutions of the starting phase of an electron which is released with some phase error for two different points in the multipactor susceptibility chart. Both are stable, but in the first, point $\mathrm{A}$, the phase converges to an equilibrium value due to the phase focusing effect (5.14), while in the second, point $\mathrm{B}$, the phase does not converge but follows a periodic structure.
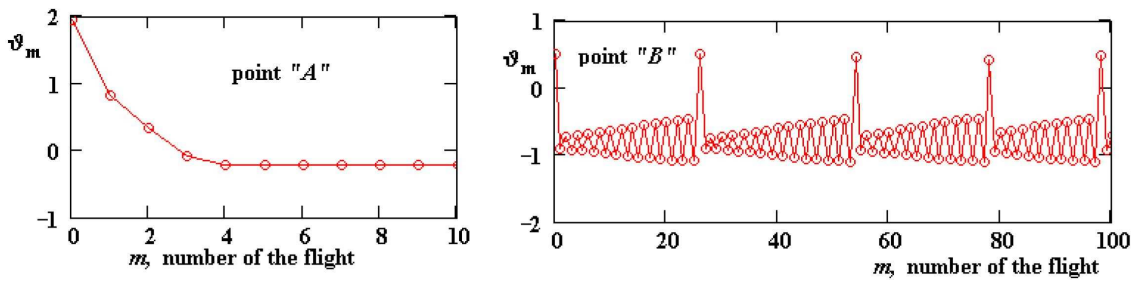

Figure 5.6. Example of two electron phase evolution for the two cases of the generalized phase stability proposed by Shemelin in [She03]. Extracted from the same paper.

Therefore, the phase focusing effect explains the existence of a multipactor discharge when the source of electrons is finite, but on the other hand implies and additional condition for the multipactor discharge. The electrons must be in resonance, with the right starting phase, with the right impact energy and, in addition, in a region of phase stability. This produces even narrower multipactor regions which are completely against experimental evidences that suggest broader multipactor region with much softer transitions [HW58]. Fig. 5.7 shows the stable regions (strictly speaking $|G|<1$ ) of the first eleven modes for the constant- $k$ and constant $-v$ theories. The stable regions represent a very narrow area compared to the complete multipactor regions. There are little differences between the two theories, being almost perfectly aligned at the bottom of the regions but 
showing a different slope for increasing $f \times d$.

\section{Figure 5.7.}

Multipactor stable regions $(|G|<1)$ for constant- $k$ (with $K=$ 5 ) and constant- $v$ (with initial energy of $1.5 \mathrm{eV})$ theories.

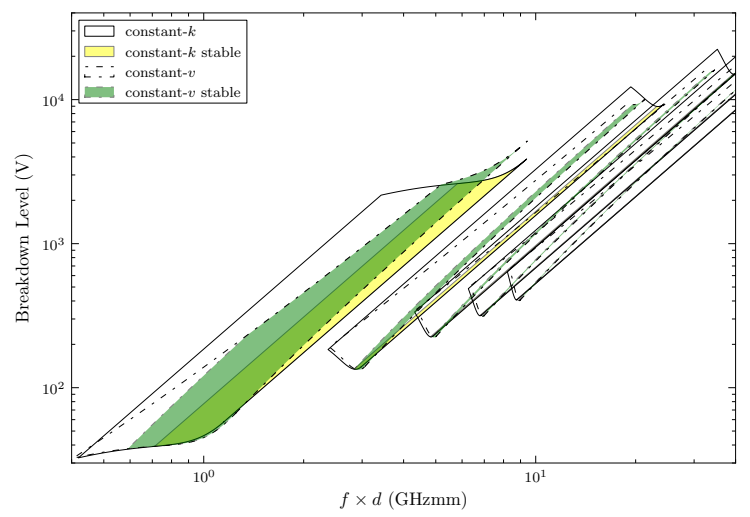

\subsubsection{Statistical Multipactor theories}

The classical theories, and all their derivations, provide a simple mathematical model for the multipactor process. These theories are very illustrative and help to understand conceptually the dynamics of the electrons in a multipactor discharge. However, they have some limitations due to excessive simplifications. The multipactor stable areas are narrow in excess and present large discontinuities that have been not observed in laboratory [HW58]. On the other hand the threshold computed with classical theories (both constant-k and constant- $v$ ) matches experimental results only for the first multipactor order, presenting a shift of all other regions to lower voltages.

The reason for these limitations is the assumption of a deterministic emission velocity, although the emission velocity is known to be random in nature with a certain distribution.

The statistical theory departs from the one-dimensional equations of motion of the classical theory presented in section 5.1.1, but assumes that the initial velocity for secondary electrons is a random variable with known probability density function.

The same as done in Section 4.3.3.1. The equations (5.3) and (5.4) are normalized dividing by $v_{\omega} / \omega$ and rewritten with the following 
notation

$$
\begin{gathered}
\xi=\omega x / v_{\omega}, \quad \varphi=\omega t, \quad \varphi_{s}=\omega t_{s}, \\
\varphi_{i}=\omega t_{i}, \quad u=v_{0} / v_{\omega}, \quad \lambda=\omega d / v_{\omega},
\end{gathered}
$$

where $v_{\omega}=\frac{e E_{0}}{m_{e} \omega}$, yielding

$$
\xi\left(\varphi, \varphi_{s}, u\right)=\left(u+\cos \varphi_{s}\right)\left(\varphi-\varphi_{s}\right)+\sin \varphi_{s}-\sin \varphi .
$$

The phase $\varphi_{i}=\omega t_{i}$ at which the electron impacts with the opposite side is the least root of equation (5.17) setting $\xi=\lambda$

$$
\lambda=\left(u+\cos \varphi_{s}\right)\left(\varphi_{i}-\varphi_{s}\right)+\sin \varphi_{s}-\sin \varphi_{i} .
$$

Equation (5.18) establishes the condition for an electron to reach the opposite plate $\xi=\lambda$ at phase $\varphi_{i}$, starting with phase $\varphi_{s}$, and initial velocity $u$.

The subtle change of considering $u$ as a random variable implies a complete different treatment of the problem. Instead of having deterministic results, statistical theories will yield a rich set of probabilities and averages of different quantities related to multipactor. In the classical theories, electrons are always released with the same fixed velocity, which implies that for a given starting phase they will always follow the same trajectory and will impact against the opposite surface always in the same transit time. On the contrary, within the statistical theories, electrons have random emission velocity and, therefore, they may follow one trajectory or another with different probability.

Fig. 5.8 below depicts this new situation. In the present example, one electron is released at a phase $\varphi_{s}$. Four possible trajectories are plotted for four different emission velocities $v_{1}>v_{2}>v_{3}>v_{4}$. We can see that, for the first three emission velocities, the electron reaches the opposite plate located at $\xi=\lambda$ in three different transit times $\tau=\varphi_{i}-\varphi_{s}$. For the lowest emission velocity, it does not even reach it and impacts back against the starting surface.

The probability density function of the emission energy for secondary electrons is known and reported in [FP02, $\left.\mathrm{CCF}^{+} 04, \mathrm{Gre} 02\right]$. The dynamics of secondary electrons depend on their nature, i.e, if it is a true secondary, elastically or inelastically backscatered electron. For simplicity, the statistical theory assumes that all secondary 
Figure 5.8.

Electron trajectories with random initial velocity. Depending on the initial velocity the electron reaches the opposite plate at different instants or it impacts back to the emission surface.

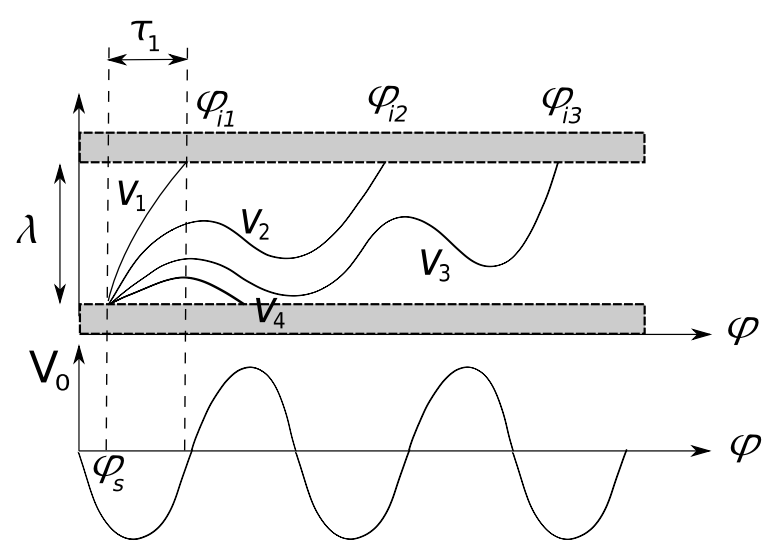

electrons belong to the true secondary species and therefore have an unique probability density function for all of them. This is a reasonable approach since the true secondary electrons are dominant for the most part of the impact energy range, except for the very low energy region (few eV), where elastic collisions are more frequent.

\subsubsection{Stationary theory}

In this section, the single-carrier two-surface statistical multipactor theory for parallel-plates, developed by Vdovicheva et al. in [VSS04], is reviewed with some detail. There are extensions of this theory dealing with dielectrics $\left[\mathrm{SSB}^{+} 05\right]$ and $2 \mathrm{D}$ motion in waveguides [SSV08], although the core of the theory is full-contained in the parallel-plates case.

The word "stationary" is not present in its original publication, although it has been included in this review in order to differentiate it from the non-stationary theory presented in this Thesis $\left[\mathrm{AVG}^{+} 10\right.$, $\mathrm{AMV}^{+}$11] (See Sections 4.3 and4.4). Concretely, the stationary adjective has been included because this theory assumes that, after many interactions, the electrons have enter in a stationary resonance in which the phase distribution of emitted electrons is invariant interaction after interaction. We will discuss this with some more detail next.

The statistical theory is constructed around the joint probability density $G\left(\tau \mid \varphi_{s} ; \lambda\right)$ which represents the probability density function 
of the transit time $\tau$ between plates separated $\lambda$ of an electron released at phase $\varphi_{s}$.

Therefore, they key point in this theory is the construction of $G\left(\tau \mid \varphi_{s} ; \lambda\right)$ given the equations of motion of (5.18) and the probability density function of $u$, namely $f_{u}(u)$. There are some analytical approximations of $f_{u}(u)$ in literature. Any of them could be applied in this theory. One of the most common is the Maxwellian distribution, which has been used mostly in this Thesis,

$$
f_{u}=\frac{u v_{\omega}^{2}}{v_{t}^{2}} \exp \left(-\frac{u^{2} v_{\omega}^{2}}{2 v_{t}^{2}}\right)
$$

According to the theorem of transformation of Univariate Random Variables [DEGL08], of the statistical theory, if $X$ is a random variable with probability density function $f_{X}(x)$ and $Y$ is a random variable which is a function of $X$, such as $Y=h(X)$ and $h$ is monotonic, the probability density function of $Y$ can be expressed as

$$
f_{Y}(y)=\left|\frac{d h^{-1}(y)}{d y}\right| f_{X}\left(h^{-1}(y)\right)
$$

Now, taking the analogies $u \equiv X$ and $\tau \equiv Y, G\left(\tau \mid \varphi_{s} ; \lambda\right)$ can be expressed as:

$$
G\left(\tau \mid \varphi_{s} ; \lambda\right)=\left|\frac{d g\left(\tau \mid \varphi_{s} ; \lambda\right)}{d \tau}\right| f_{u}\left(g\left(\tau \mid \varphi_{s} ; \lambda\right)\right),
$$

where $u=g\left(\tau \mid \varphi_{s} ; \lambda\right)$ must be a monotonic function which express $u$ as a function of $\tau$. The function $g\left(\tau \mid \varphi_{s} ; \lambda\right)$ can be easily derived from (5.18), where the non-monotonic regions must be removed. See [VSS04, $\left.\mathrm{AVG}^{+} 10\right]$ or Section 4.3 for more details on this operation.

Now, let us introduce another definition. $\Phi\left(\varphi_{s}\right)$ represents the probability that an electron is released at starting phase $\varphi_{s}, \varphi_{s} \in$ $[0,2 \pi]$. Such a probability depends on the emission velocity distribution, the transit time and secondary emission yield of all previously emitted electrons. In general, $\varphi_{s}$ is a non-stationary process, i.e. starting from a given initial distribution at $t=0, \Phi\left(\varphi_{s}\right)$ changes with time (see section 4.3.3).

Based on the previous definitions of $G\left(\tau \mid \varphi_{s} ; \lambda\right)$ and $\Phi\left(\varphi_{s}\right)$, Vdovicheva defines a set of parameters which characterize the statistical behavior of the multipactor discharge. These parameters are 
Table 5.1. Statistical definitions.

Probability of impacting against the opposite wall with a transit time $\tau$, for an electron with starting time $\varphi_{s}$

Probability that an electron is emitted at starting phase $\varphi_{s}$

Secondary Emission Yield for an electron emitted at starting $\sigma\left(\tau \mid \varphi_{s} ; \lambda\right)$ phase $\varphi_{s}$ and reaching the opposite wall in a transit time $\tau$ (deterministic value)

Probability that an electron emitted at starting phase $\varphi_{s}$ impacts with opposite wall

$$
p\left(\varphi_{s}, \lambda\right)=\int_{0}^{\infty} G\left(\tau \mid \varphi_{s} ; \lambda\right) d \tau
$$

Fraction of electrons (emitted in one period of the rf signal)

$$
\eta(\lambda)=\int_{0}^{2 \pi} d \varphi_{s} \Phi\left(\varphi_{s}\right) \int_{0}^{\infty} G\left(\tau \mid \varphi_{s} ; \lambda\right) d \tau
$$

impacting with opposite wall

Effective coefficient of secondary electron emission

$$
\sigma_{e f f}(\lambda)=
$$

$$
\frac{1}{\eta(\lambda)} \int_{0}^{2 \pi} d \varphi_{s} \Phi\left(\varphi_{s}\right) \int_{0}^{\infty} G\left(\tau \mid \varphi_{s} ; \lambda\right) \sigma\left(\tau \mid \varphi_{s} ; \lambda\right) d \tau
$$

summarized in Table 5.1, see [VSS04] for a detailed mathematical analysis.

Note that $\sigma\left(\tau \mid \varphi_{s} ; \lambda\right)$ has a deterministic value and can be easily calculated from the impact energy [using (5.3) with normalized units] and applying one of the available SEY models in literature. The condition of the multipactor discharge is $\eta \sigma_{\text {eff }} \geq 1$, which means that the number of created electrons is higher than the number of impacting electrons. 
The definitions of Table 5.1 constitute the basis for the statistical analysis.

In order to calculate $\Phi\left(\varphi_{s}\right)$, Vdovicheva makes two relevant assumptions which simplifies the problem but also limits the range of application of the theory:

1. The multipactor process is stationary. After a number of interactions $(l)$, only the electrons emitted at favorable phases multiplicate, and therefore the distribution $\Phi_{l}\left(\varphi_{s}\right)$, converges to a stationary phase distribution,

$$
\lim _{l \rightarrow \infty} \Phi_{l}\left(\varphi_{s}\right)=\Phi_{s t}\left(\varphi_{s}\right) .
$$

2. The electron population does not variate in time, thus there is no exponential growth nor absorption. This implies that $\eta \sigma_{e f f}=1$.

Next, Vdovicheva find the relationship between the phase distributions of two consecutive interactions $l$ and $l-1$ and applies the above assumptions. The condition of stationarity implies

$$
\Phi_{l-1}\left(\varphi_{s}\right)=\Phi_{l}\left(\bmod \left(\varphi_{s}+\pi ; 2 \pi\right)\right) .
$$

Here, $\bmod (a, b)$ stands for the modulo operator (or remainder of $a / b)$. Note that two consecutive interactions occur at opposite plates and reversing normal emission direction. Therefore their phases are related by a relative shift of $\pi$ radians.

Finally, adding the condition stable population $\eta \sigma_{e f f}=1$, the following equation is derived (see [VSS04] for a detailed analysis).

$$
\Phi_{s t}\left(\varphi_{s}\right)=\int_{0}^{2 \pi} K\left(\varphi_{s} \mid \varphi_{s}^{\prime} ; \lambda\right) \Phi_{s t}\left(\bmod \left(\varphi_{s}^{\prime}+\pi ; 2 \pi\right)\right) d \varphi_{s}^{\prime}
$$

where

$$
K\left(\varphi_{s} \mid \varphi_{s}^{\prime} ; \lambda\right)=\sum_{n=0}^{\infty} G\left(\varphi_{s}-\varphi_{s}^{\prime}+2 \pi n \mid \varphi_{s} ; \lambda\right) \sigma\left(\varphi_{s}-\varphi_{s}^{\prime}+2 \pi n \mid \varphi_{s} ; \lambda\right) .
$$


Rearranging the limits of integration, (5.23) can be rewritten as

$$
\begin{aligned}
& \Phi_{s t}\left(\varphi_{s}\right)= \\
& \alpha \int_{0}^{2 \pi} K\left(\varphi_{s} \mid \bmod \left(\varphi_{s}^{\prime}+\pi ; 2 \pi\right) ; \lambda\right) \Phi_{s t}\left(\varphi_{s}^{\prime}\right) d \varphi_{s}^{\prime},
\end{aligned}
$$

which has the form of a homogeneous Fredholm equation of the second kind and can be solved with standard numerical techniques. The scalar $\alpha$ is equal to $\left(\eta \sigma_{e f f}\right)^{-1}$ and has been added with respect to the original formulation of Vdovicheva. This term can be easily derived from the Vdovicheva's development but, since the author imposes the condition of stability $\left(\eta \sigma_{e f f}=1\right)$, it dissapears in its definitive form. We will keep it here, since it renders the equation (5.24) valid also for a resonant situation with an exponential growth of the electron population $\left(\eta \sigma_{e f f}>1\right)$. A similar approach is adopted by a later work of Sazontov et al. in [SSV08], where the exponential growth is defined equivalently as $\exp (\gamma)=\eta \sigma_{e} f f$.

The solution of (5.24) yields the minimum characteristic value $\alpha_{\min }$ (and the associated probability density function $\Phi_{s t, m i n}$ ) which depending on its value defines the criterion of multipactor discharge.

- $\alpha_{\min }>1$. This means that $\eta \sigma_{e f f}<1$, the electrons are absorbed, and therefore there is no multipactor discharge. Since there is not any favorable phase distribution for multiplication the assumption of stationarity is violated rendering $\Phi_{s t, \min }$ meaningless.

- $\alpha_{\min }=1$. This implies that $\eta \sigma_{e f f}=1$ and the electron population remains stable. This solution sets the threshold for the multipactor breakdown. the stationarity condition is fullfilled and $\Phi_{s t, m i n}$ is the solution of the phase probability density.

- $\alpha_{\min }<1$. There is a multipactor discharge with exponential electron growth $\left(\eta \sigma_{e f f}>1\right) . \Phi_{s t, m i n}$ is the solution of the phase probability density.

The solution of (5.24) allows plotting the multipactor breakdown regions in $f d$ versus $V$ charts, yielding much more realistic curves than the classical theory. See the example of Fig. 5.9, for emission 
velocity distribution of (5.19) with electron emission energy of $1.5 \mathrm{eV}$, the SEY model of $\left[\mathrm{VMW}^{+} 05 \mathrm{a}, \mathrm{AVG}^{+} 07\right]$ and ECSS silver parameters given in [EST03].

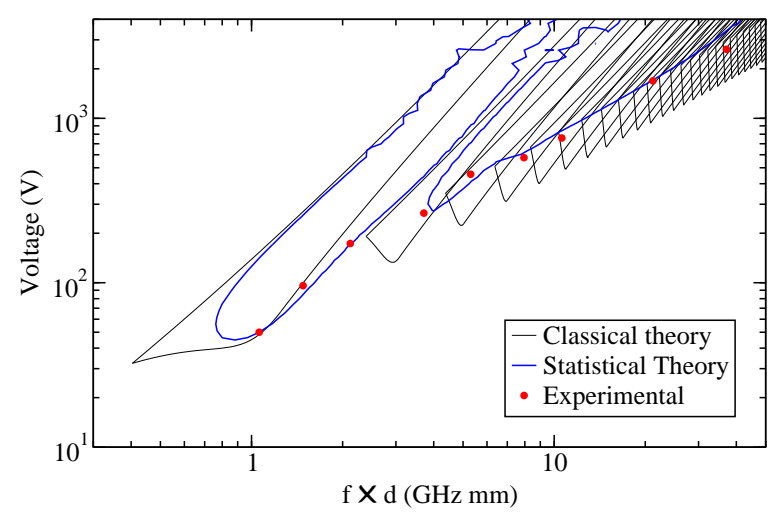

Figure 5.9.

Comparison of multipactor boundaries computed with different theories. Experimental data from [WJ89] has been added.

Therefore, the stationary statistical theory presented by Vdovicheva sets an excellent framework for the Multipactor statistical theory although it has some limitations:

- First, according to Vdovicheva's initial work in [VSS04], the theory is only able to determine the existence or not of multipactor discharge, but it is not able to predict the speed of growth of the discharge in time. A further development in $\left[\mathrm{SVB}^{+} 03, \mathrm{KLS}^{+} 08\right]$ gives the expression for the exponential growth at each interaction, but it does not give any information about the periodicity of the interactions in time.

- On the other hand, this theory does not model exactly the polyphase multipactor as [VSS04, $\mathrm{KLS}^{+} 08$ ] claim, since it only takes into account double surface impacts (the electron is released from one plate and impacts against the opposite one). The eventual single surface impacts are neglected, which is equivalent to assume that the impact energy of all single surface impact yields a SEY equal to 0 .

- Finally, since the theory assumes stationarity, it is not valid for modelling electron absorption rates at regions below breakdown. 


\subsubsection{Non-stationary theory}

The non-stationary theory is the core of the theoretical development of this Thesis and has been already published for both single and multi-carrier signals in $\left[\mathrm{AVG}^{+} 10\right]$ and $\left[\mathrm{AMV}^{+} 11\right]$, respectively. Such papers have been included in this Thesis in Sections 4.3 and 4.4, respectively. Please refer to them for a detailed theoretical analysis.

The non-stationary theory belongs to the statistical theories and shares some of the formulation with the stationary one. More specifically, the derivation of the $G\left(\tau \mid \varphi_{s} ; \lambda\right)$ function, referred as Transit Time Probability Distribution (TTPD), is similar and constitutes the core of both theories.

However, the non-stationary theory has some differences with respect to the stationary one which implies some advantages:

- It generalizes the TTPD function to cover both single and twosurface interactions. It allows the non-stationary theory for incorporating single surface impacts within the multipactor process which can not be done with the stationary one.

- It extends the time window from one period of the envelope, as the stationary theory does, to infinity. This removes the stationarity from the problem and allows modeling other than stationary multipactor processes. For example, the stationary theory can not model the rate of absorption of electrons below the multipactor threshold, whereas the non-stationary theory is able to do it. As a counterpart, this change in time span implies that, instead of solving a single Fredholm integral equation as in the stationary case, a Volterra system of equations must be solved. There is a slight increase in the problem complexity, but it is not significant if solved numerically as it is done in most real cases.

- The non-stationary theory has been successfully formulated for the multi-carrier case, being the first full theory for multipactor in multi-carrier signals. The stationary theory could also be reformulated to the general multi-carrier case, although the lack of single surface interactions in the theory might not render it suitable for such cases. 
- The non-stationary theory offers a set of indicators that better characterize the time dynamics of the multipactor phenomenon than the stationary case, including rate of impacts, instantaneous SEY and instantaneous multipactor order.

Compared to the stationary theory of Section 5.1.2.1, the nonstationary theory renders more precise single-carrier multipactor boundaries thanks mainly to the inclusion of single-carrier impacts. See the example of Fig. 5.10, for emission velocity distribution of (5.19) with electron emission energy of $1.5 \mathrm{eV}$, the SEY model of $\left[\mathrm{VMW}^{+} 05 \mathrm{a}, \mathrm{AVG}^{+} 07\right.$ ] and ECSS silver parameters given in [EST03]. The non-stationaty theory match better the numerical results computed with commercial FEST3D [FES] than the stationary one, specially for the upper boundary of the multipactor first-order region where high fields increase the rate of single-surface interactions.

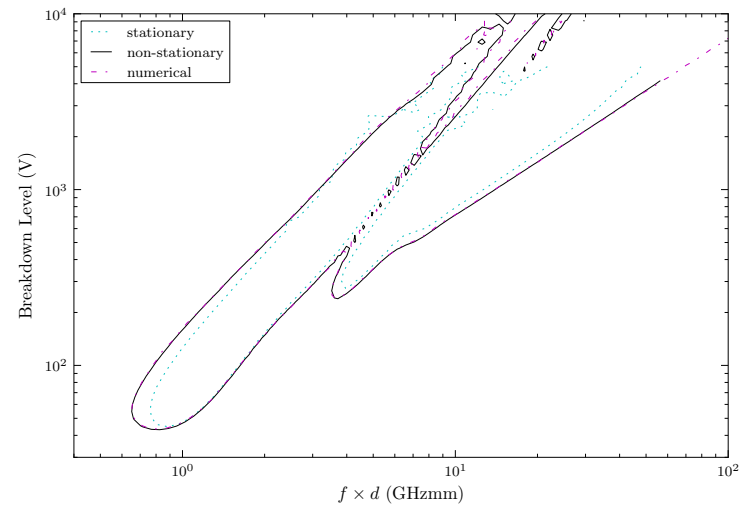

Figure 5.10.

Stationary vs nonstationary comparison. Numerical simulations done with FEST3D software have been also included.

\subsubsection{Single-carrier prediction}

In practical situations, the purpose of every multipactor theory is to be able to provide accurate predictions of the threshold power in order to design multipactor-free components and avoid the need of testing following a try-and-error basis. The uncertainty in the predictions makes necessary the imposition of some design margins in order to ensure that the device is multipactor free. The higher the uncertainty of the prediction tool, the higher the necessary margins are. 
Figure 5.11.

Classical theories are plotted against stationary and non-stationay statistical theories. Experimental values are also shown.

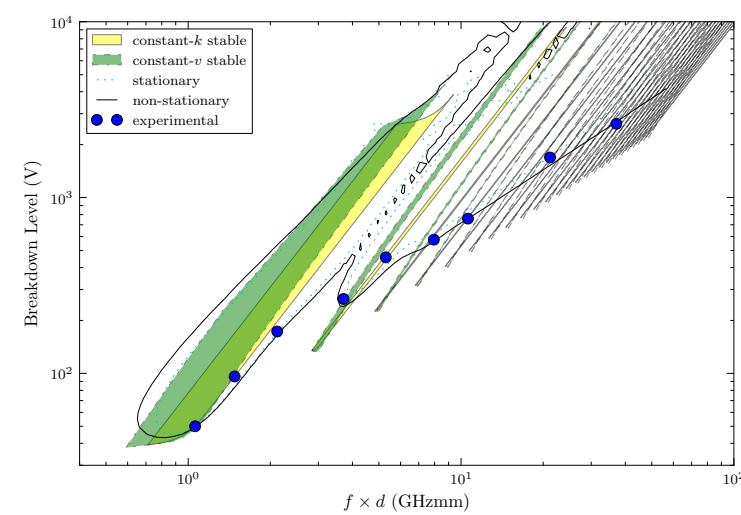

Regarding the single-carrier theories, the classical theories (constant- $k$ and constant- $v$ ) provide accurate prediction only for the first multipactor mode. For the rest of them, the stability condition produces extremely narrow regions which are not in agreement with experimental results. Furthermore, the lowest part of the regions is overally down-shifted with respect to the experimental results. On the contrary the statistical theories match reasonably well the experimental results for all multipactor orders. The statistical theories do not show such discontinuities between regions but rather a multipactor region spread producing overlap and a smooth transition between regions. The non-stationary boundary is slightly more precise than the stationary one due to the inclusion of single-carrier interactions.

\subsection{Multi-carrier theories}

There was little previous work in literature about multi-carrier signals. In fact, there was not a complete theory dealing with multipactor in multi-carrier signals up to the development of the nonstationary theory in this Thesis. Due to the lack of a reliable theory, Industry made an effort to roughly characterize multi-carrier discharges using the single-carrier theory and making very simplistic assumptions. The result was some simple rules for predictions: Peak power rule and $p$-gap-crossing rule (see Section 2.4.3.1). These rules provide very inaccurate predictions which are compensated by large 
margins in the design. These margins render these rules safe but conservative in excess with the related increase in mass, size and cost budgets. This Thesis provides a new multipactor theory for multicarrier signals: The non-stationary theory. Such a new theory is able to accurately predict multipactor behavior under arbitrary signals which allows for predicting multipactor in multi-carrier components with a higher degree of accuracy with respect to the previous simplistic approaches.

\subsubsection{Peak power}

This rule is very simple. If the the peak voltage of the multi-carrier signal is higher than the single-carrier multipactor threshold (computed with any of the existing prediction techniques in single-carrier signals), there is multipactor. For a multicarrier signal of $K$ carriers with amplitudes $V_{i}, i=1,2, \ldots, K$, the peak voltage is simply

$$
V_{p}=\sum_{i=1}^{K} V_{i} .
$$

In terms of power, the peak power becomes

$$
P_{p}=\left(\sum_{i=1}^{K} \sqrt{P_{i}}\right)^{2} .
$$

For equal amplitude carriers, $V_{i}=V_{0}$, and $P_{i}=P_{0}, \quad \forall i$, it finally becomes, $V_{p}=K V_{0}$ and $P_{p}=K^{2} P_{0}$. Hence it is also called the $K^{2}$ rule.

One open question in this rule is what reference frequency should be taken for the single-carrier threshold computation. Usually, the lowest frequency of the train of carriers is taken in order to be even more conservative [EST03], although, according to $\left[\mathrm{AMV}^{+} 11\right]$ (see Section 4.4), it would be more reasonable to take the center frequency instead.

This rule disregards completely the possible phase combinations of the carriers within the multi-carrier signal. As already mentioned throughout this Thesis, the phase combination determines the shape of the envelope. The peak power rule assumes the highest level of 
the envelope, corresponding to the peak voltage, and hence it constraints the multi-carrier signal to be always below the single-carrier threshold. It therefore represents the worst possible case, being the most conservative rule of all existing ones.

\subsubsection{0-gap-crossing rule}

The 20-gap-crossing rule belongs to the family of the $p$-gap-crossing rules. The same as the peak power one, these rules take the singlecarrier threshold as a reference and assume that, when the multicarrier envelope is above such a limit, there is multipactor, and below it, there is not. The difference is that the $p$-gap-crossing rules consider that the multipactor discharge is not instantaneous and that it needs some build-up time. Therefore, these rules allow the multi-carrier signal to be above the single-carrier threshold for some amount of time before the discharge takes place. The time that the envelope can exceed the threshold is measured in number of gap-crossings or interactions of electronic bunch with the surfaces of the device. This is what the $p$ of the name stands for. For instance, the 5-gap-crossing rule, $p=5$, establishes that there will be multipactor only when the multi-carrier envelope is above the threshold for a time equivalent to 5 electron gap crossings. Therefore, in this case it is assumed that 5 electron interactions are enough to produce a multipactor discharge. The $p$-gap-crossing rules imply a relaxation with respect to the peak power rule, which can be considered equivalent to a 0-gap-crossing rule (since it does not allow the signal to be above the single-carrier threshold for any time).

The classical multipactor theory is used to determine the length of the interval above threshold necessary to have $p$ interactions

$$
T_{p}=\frac{p n}{2 f},
$$

where $n$ is the multipactor order and $f$ is the single-carrier frequency. The same as the peak power rule, the lowest frequency of all carriers is usually taken as a reference.

The key question in these rules is what number of gap-crossings is necessary to have a multipactor discharge. There are not scientific evidences to establish a fixed number as an universal criterion. Industry uses one number $p$ or another based on own experiences and 
depending on the safety margin that one wants to apply. Therefore, different criteria are used for each particular case, ranging from $p=4$ to $p=20$.

The 20-gap-crossing rule has become popular because it comes from a study done in ESA/ESTEC [MMS ${ }^{+}$97] that fixes the number of gap-crossings to $p=20$ based on numerical simulations and some experimental tests.

Even if no theory or strong evidences justify such a number, for practical considerations, the 20-gap crossing rule has been widely adopted because it is thought to be conservative in all cases [WSS00]. However, there are not evidences that this is always true. For example, in Mader $\left[\mathrm{MPD}^{+} 03\right.$ ], the 20-gap-crossing rule has been employed in simulations of 3D complex structures and experimentally tested, showing, in this case a good agreement but highlighting that the 20gap-crossing prediction yielded a higher breakdown power than the test result. This is one case in which the 20-gap-crossing rule is not conservative.

In fact, the 20-gap-crossing rule is based exclusively on numerical simulations of a limited number of multicarrier signals, with only in-phase (or zero phase) schemes. The experimental results of $\left[\mathrm{MMS}^{+} 97\right]$ did not contradict the rule, but as the authors say: "Moreover, experimental data are only available for 1st and 3rd order multipaction gaps. It would be advisable to test larger gaps before adopting the above rule in the design of components". Until the beginning of this Thesis, no other experimental results had verified yet the validity of the 20-gap-crossing rule. For example, experimental results of [GW96] yield a minimum number of gap-crossings of 38 instead of 20 .

From a physical point of view, it does not seem logical to establish a fixed number of gap crossings as a multipactor criterion for all situations, since depending on the impact energy or material SEY more or less secondary electrons could be produced. Also, more variables come in play if also considering accumulation effects between consecutive periods of the multi-carrier envelope.

The $p$-gap-crossing rules have an implication on the envelope. In fact, the breakdown voltage or power is computed by setting the length of the main lobe of the envelope to $T_{p}$, from (5.27), with a height equal to the single-carrier threshold. This is usually done by 
optimizing the phases of the multi-carrier signal (with some kind of algorithm) in order to conform the envelope to a square shape [ECS]. An alternative is to use approximated boundary curves such Angevain's [ADDM09] and Wolk's [WSS00] ones, which also consider the multi-carrier envelope as a square signal.

On the other hand, the multi-carrier envelope is periodic with $T_{e}$ period and is theoretically bounded by two limits: the peak voltage and the average voltage (or peak power and average power). Fig. 5.12 shows an example for eight carriers.

\section{Figure 5.12.}

Example of multicarrier signal envelope for the case of eight carriers, with a uniform frequency separation of $\Delta f=50 \mathrm{MHz}$.

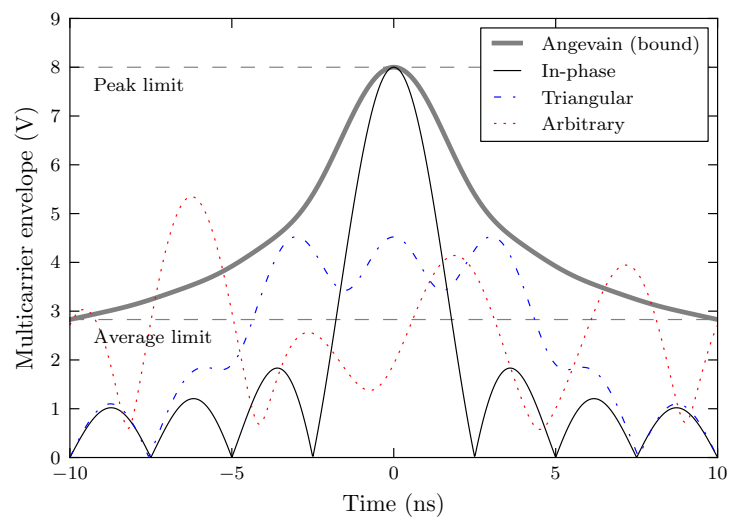

Note that the value of $T_{p}$ given by (5.27) depends on the multipactor order $n$ and the single-carrier reference frequency $f$ and that, on the other hand, the period of the envelope $T_{e}$ depends exclusively on the differences between the frequencies of the carriers (see $\left[\mathrm{AMV}^{+} 11\right]$, Section 4.4). Therefore, for a fixed $p$, the ratio $T_{p} / T_{e}$ varies for each different case. There are two limit cases:

- If $T_{p} / T_{e} \approx 0$ the envelope resulting from optimization will be a in-phase one with a maximum value equal to the peak voltage value. In this case the $p$-gap-crossing rule is equivalent to the peak power rule and it is most conservative case.

- If $T_{p} / T_{e} \geq 1$ the result will be an almost flat envelope or continuous wave (CW) (depending on the number of carriers) with a value close to the average voltage value. In this case the $p$ - 
gap-crossing rule represents the more optimistic case and the less conservative one.

As an example, let us consider the popular 20-gap-crossing rule and evenly spaced multi-carrier signals with $\Delta f$ separation, for which $T_{e}$ has a simple expression $T_{e}=1 / \Delta f$. For a reference frequency for single-carrier of say $10 \mathrm{GHz}$ (the frequency of the first carrier) and a device gap of $1 \mathrm{~mm}$, the multipactor order will be of $n \approx 7$ for typical materials. According to (5.27) this implies a $T_{20}=7$ ns. now consider two cases in which the carriers are separated $\Delta f=10 M h z$ and $\Delta f=$ $200 M h z$, respectively. The period of the envelope will be different with $T_{e}=100 \mathrm{~ns}$ and $T_{e}=5 \mathrm{~ns}$, which implies a $T_{20} / T_{e}=0.07$ and $T_{20} / T_{e}=1.4$. Therefore, in the first case, the 20-gap-crossing rule will yield a result similar to the peak power rule and in the second case it will yield a much more optimistic case close to the average voltage value. Fig. 5.13 shows the signal optimization according to the 20-gap-crossing rule for the two cases.

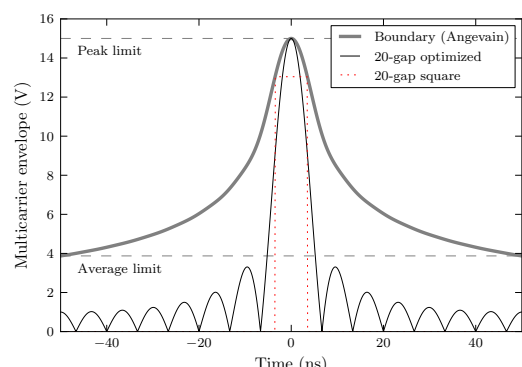

(a) $\Delta f=10 \mathrm{MHz}$

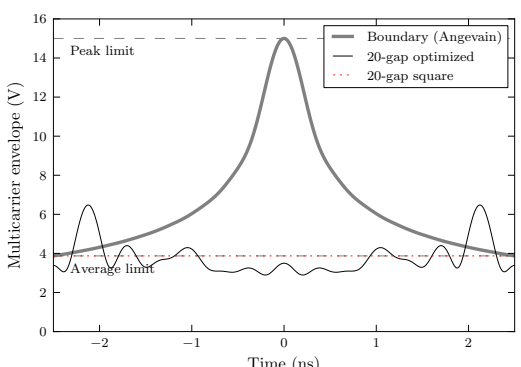

(b) $\Delta f=200 \mathrm{MHz}$.

Figure 5.13. Example of multi-carrier signal envelope for the case of fifteen carriers. Two different frequency separations are used. The phases have been optimized in both cases to fit the 20-gap-crossing rule.

For the special case of $K$ carriers with equal separation $\Delta f$

$$
\frac{T_{20}}{T_{e}}=10 n\left(\frac{\Delta f}{f}\right),
$$

being a function of solely $n$ and $\Delta f / f$. 
As stated above, values of $T_{20} / T_{e} \geq 1$ correspond to the CW case, whereas values obeying $T_{20} / T_{e} \leq 1 / K$ will yield in-phase optimizations. With (5.28) and applying such limits, an illustrative 20 -gap-crossing rule prediction map can be computed, as shown in Fig. 5.14. Colored area represents the $\mathrm{CW}$ region. All multi-carrier signals with $\Delta f / f$ and multipactor order $n$ inside this area will have 20 -gap-crossing rule predictions tending to a flat envelope. In turn, the different lines represent the in-phase limit for different $K$. Signals below such limit will have 20-gap-crossing rule predictions equal to the peak-power or in-phase cases.

Therefore, this map can be used to classify what kind of prediction is going to yield the 20-gap-crossing rule for each specific case: a CW prediction or a peak power one.

An interesting conclusion is that a $p$-gap-crossing rule, no matter the value of $p$, can represent sometimes the worst case or sometimes the most optimistic one, depending on the combination of frequencies and device gap of each real case.

\section{Figure 5.14.}

Map of 20-gap-crossing rule predictions. It represents the ratio $T_{20} / T_{e}$ for different combinations of parameteres. CW (colored area) and in-phase limits (lines) are represented.

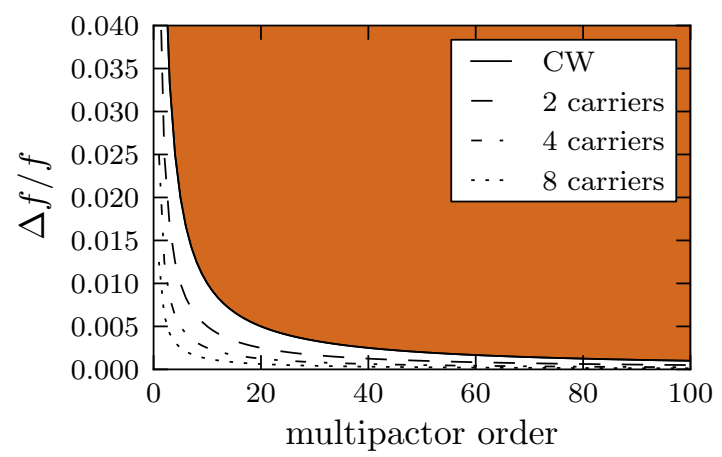

\subsubsection{Non-stationary multi-carrier theory}

The non-stationary theory for multi-carrier signals presented in this Thesis $\left[\mathrm{AMV}^{+} 11\right]$, Section 4.4, is a generalization of the original nonstationary one which is also part of this work $\left[\mathrm{AVG}^{+} 10\right]$, Section 4.3. The Non-stationary multi-carrier theory is the first theory which is able to fully characterize the multipactor phenomenon for a com- 
pletely arbitrary multi-carrier signal. The whole theory has been already presented and covered in 4.4. For more details on the theory please refer to the aforementioned Section.

The same as its single-carrier predecessor, the non-stationary theory for multi-carrier signals considers both two-surface and singlesurface events and it is able to reproduce the electron generation and absorption above and below the breakdown threshold. Hence, this theory is able to accurately reproduce the electron growth during the "On" and "Off" intervals of the multi-carrier envelope.

\subsubsection{Quasi-stationary theory}

The quasi-stationary theory is a simplification of the electron dynamics in a multi-carrier discharge. The electron growth and absorption during the period of the multi-carrier envelope are approximated by a sophisticated model which is based on the single-carrier non-stationary theory.

The Quasi-stationary theory takes the center frequency of the train of carriers as a reference instead of the lowest one. As presented in $\left[\mathrm{AMV}^{+} 11\right]$ (see Section 4.4), the instantaneous frequency of the multi-carrier signal oscillates periodically around the center frequency. In addition, it can be demonstrated that the instantaneous frequency of a multi-carrier signal of equally spaced carriers and zero-phase (in-phase carriers) is constant and equal to the center one (see Appendix B).

Compared to the nonstationary multi-carrier theory, the quasistationary one is also able to model the electron population during the period of the multi-carrier envelope, although is less accurate, since it makes some approximations. In practice, both multi-carrier and quasi-stationary theories must be solved numerically, but the quasistationary theory is much less complex and faster to solve, since it is based in the single-carrier theory and has a considerable lower number of equations to solve. Therefore, this renders the quasi-stationary theory optimal for massive computation of multipactor discharges, for example, when seeking for the combinations of phases which renders the lowest breakdown power per carrier through some kind of numerical optimizer. 
Hence, the quasi-stationary theory is part of this Thesis and has been proposed in $\left[\mathrm{AVG}^{+} 12\right]$ for the use of numerical optimization of the phases of the multi-carrier signal, where computational speed is a must. See Section 4.5 for details on this theory.

\subsubsection{Multi-carrier prediction: Worst-case determination}

As seen in Section 5.1.3, for the single-carrier case, the prediction gives only a value for the breakdown voltage (power), given the geometry of the device, the frequency of operation and the material SEY properties.

The situation in the multi-carrier counterpart is dramatically different. The multipactor discharge in multi-carrier signals depends not only on the amplitude of each of the different carriers, but also on the relative phase among them. This means that for the same device, material and carrier frequencies, a certain combination of phases implies a higher or lower breakdown level than for the others. For a signal of $K$ carriers, the multipactor prediction should give $2 K$ quantities corresponding to the amplitude and phases of all carriers that provokes multipactor with the lowest power. This is referred as the Worst-case. In most cases the amplitude of all carriers might be consider equal and therefore the number of unknowns decreases to $K+1$, corresponding to the phase value for each carrier and one single value for the threshold level for all carriers.

The different methods/theories reviewed in the above sections provide different ways to compute such a worst-case. First, the peak power method of Section 5.2.1 does not actually look for the worstcase but imposes one by definition. According to this method, the worst-case is given by the in-phase scheme (all carriers are in-phase) and the breakdown voltage/power is

$$
P_{0}=P_{S C} / K^{2},
$$

being $K$ the number of carriers and $P_{S C}$ the single-carrier threshold.

The $p$-gap-crossing rules do not provide a direct way to compute the worst-case. They provide the length of the "On" interval of the multi-carrier envelope to produce $p$ gap crossings, $T_{p}$. Once $T_{p}$ is known there are different ways to compute the worst-case which are 
not specified by the rule itself. Basically, there are two ways to proceed:

- To apply analytical boundaries to the envelope. These analytical boundaries allow for easily deriving the breakdown power given $T_{p}$ without the need to compute the phases. As a drawback these curves are less accurate and add extra margin to the predictions. Two typical boundaries are those proposed by Angevain [ADDM09] and Wolk [WSS00]. Also, only the breakdown level comes out from this method. If the phases are needed to, for example, apply them in a multipactor test, it is necessary to perform a phase optimization as explained in next point.

- To conform the multi-carrier envelope to the desired shape according to $T_{p}$. This is done through numerical optimization of the phases, selecting some optimizer algorithm and goal function. The $p$-gap-crossing rules do not specify anything about this. Typically global optimizers are used and the goal function computes the resemblance of the envelope to a square signal of length $T_{p}$ [ECS]. The output of these optimizers are the phases of each carrier and the amplitude of all carriers.

With respect to the theories developed within this Thesis a different approach has been taken. Both multi-carrier theory and quasistationary theory are able to compute the electron population during a period of the envelope for a certain phase combination. The criterion to establish that there is multipactor or not is the electron accumulation or long-term discharge $\left[\mathrm{AVG}^{+} 07\right]$. Therefore, both theories are able to determine if, for each phase combination and amplitude, there is multipactor discharge or not. There is no need for defining any number of gap crossings anymore. Given this capability, it is easy to optimize the phases to find the global minimum of a certain goal function representing the phase combination that leads to the lowest breakdown level. The selection of one global function or another is crucial for the correct convergence of the algorithm. In $\left[\mathrm{AVG}^{+} 12\right]$ a goal function is proposed for the worst-case determination of multicarrier signals (see Section 4.5. Figs. 5.15-5.17 show a conceptual 
schematic of the optimization process with the p-gap-crossing rule and the multi-carrier theories.

\section{Figure 5.15.}

Multi-carrier optimizaton process following the 20-gap-crossing rule.
Input:
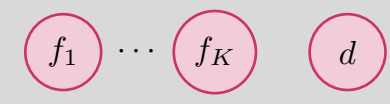

20-gcr:
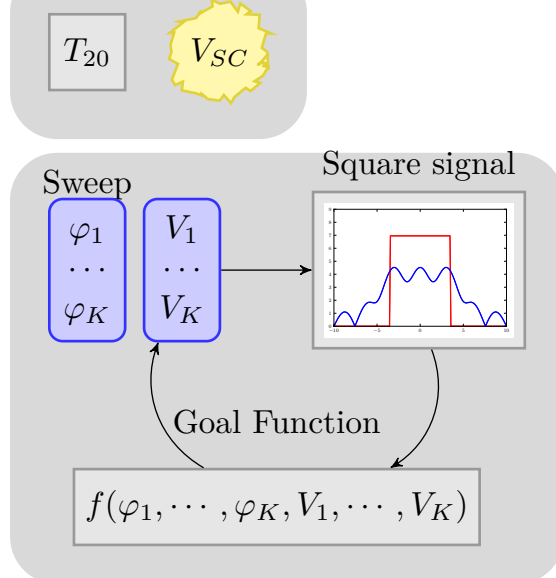

Output:

(optional)

\section{Optim:}

(optional)

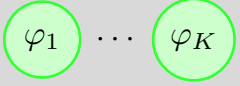

The three of them need in last instance to perform a numerical optimization. The main difference between them is that, whereas the goal function of the $p$-gap-crossing rules just consists of adjusting the envelope to a square function (the goal function could be simply to compute the square error between the envelope and a square signal), both multi-carrier theories define a goal function based on the real behavior of the electron population in the discharge. Thus, the phase optimization with the multi-carrier theories implies that for each step in the optimization process the electron growth must be solved. The number of points to be solved in a global optimization is approximately of the order of $O\left(c^{K}\right)$ which implies a vast number of computations that increase exponentially with the number of carriers 


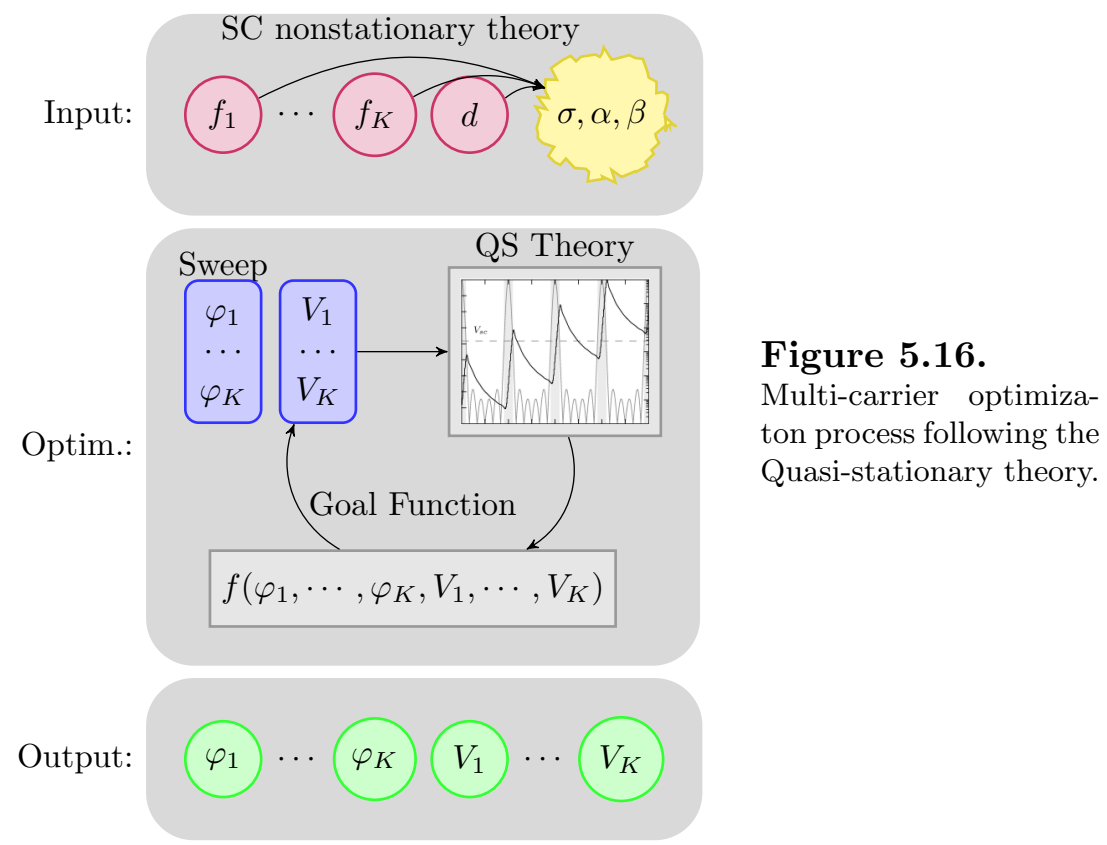

$K$. The faster resolution of the quasi-stationary method compared to the full multi-carrier theory is an advantage for practical problems where the number of carriers is a considerable high number (above $3)$.

The purpose of the optimizer is to find the phases that lead to the minimum breakdown voltage. The election of a goal function whose global minimum corresponds to this case is crucial. A possible goal function has been proposed in $\left[\mathrm{AVG}^{+} 12\right]$ (Section 4.5). Finding such a worst case may not be an easy task, since many local minima may be present. As an example, Figs. 5.18-5.20 show the breakdown voltage versus phase for different multi-carrier signals in which symmetric phase schemes have been adopted in order to be able to represent the plots in one and two dimensions for easy understanding. In general, these functions have $K$ dimensions and cannot be graphically represented.

Three cases are represented. First, a 3 carrier signal with symmetric phase distribution (only one degree of freedom). The carriers are 


\section{Figure 5.17.}

Multi-carrier optimizaton process following the full non-stationary multi-carrier theory.

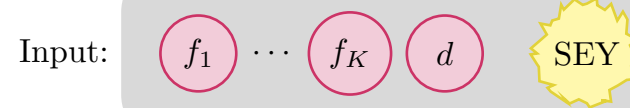

Optim.:

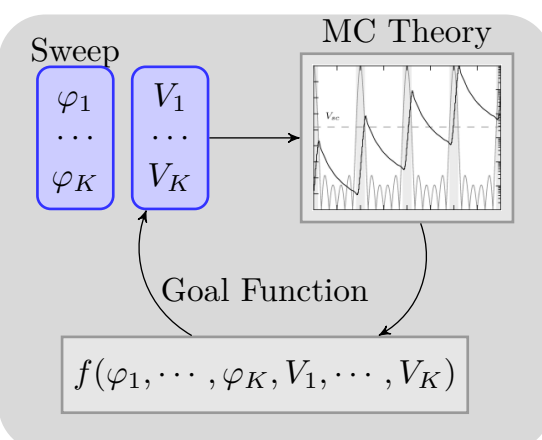

Output: $\varphi_{1} \cdots \varphi_{K} V_{1} \cdots V_{K}$
Figure 5.18.

Example of voltage breakdown vs phase for 3 carrier signal with symmetric phase distribution. The carriers are separated, $\Delta f=50 \mathrm{MHz}$ and the first frequency is $f_{1}=11.06 \mathrm{GHz}$. The gap is set to $0.6 \mathrm{~mm}$, and the material is ECSS silver [EST03], being the multipactor order of 5 .

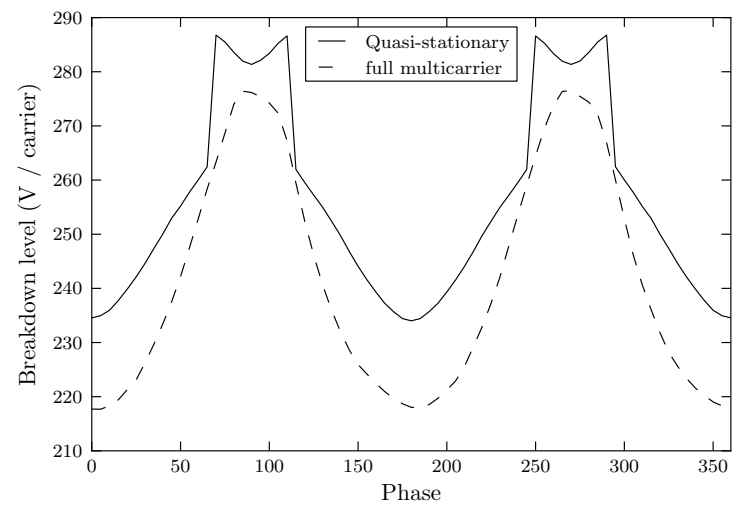



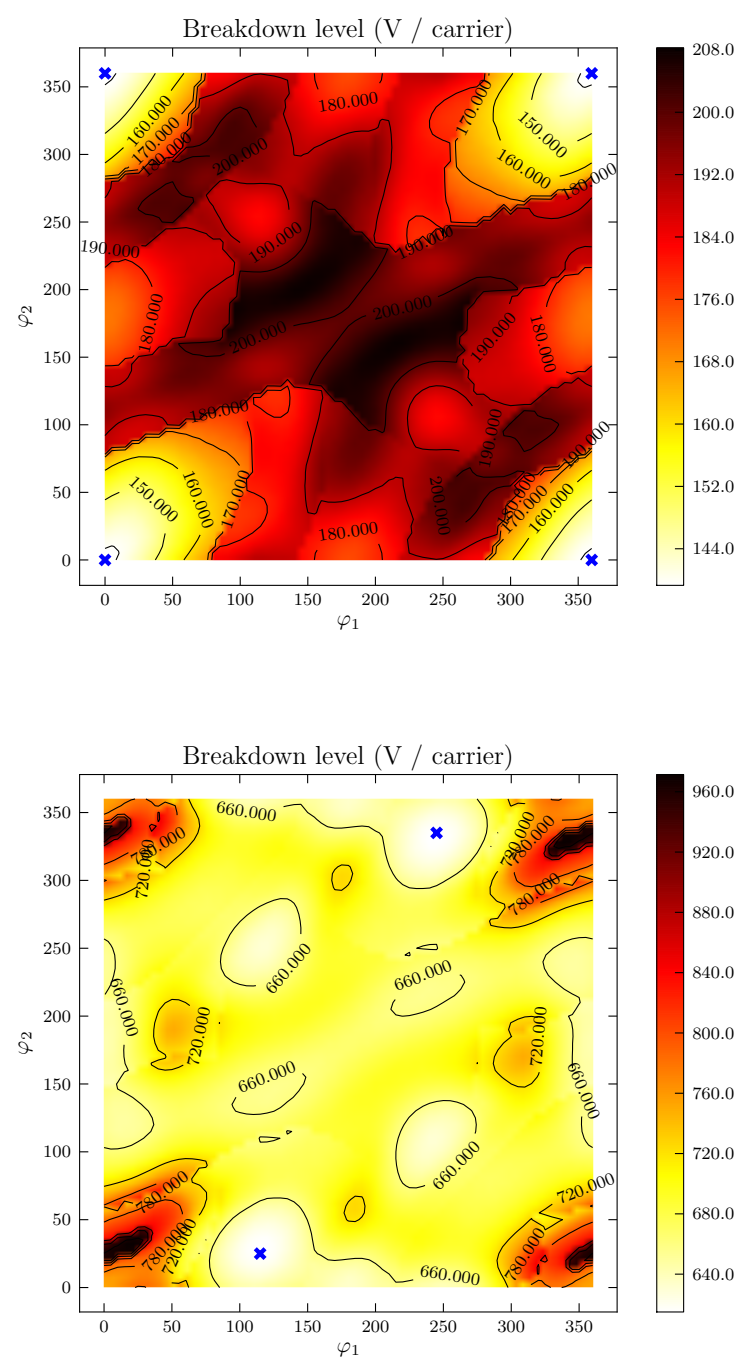

Figure 5.19.

Example of voltage breakdown vs phase for 6 carrier signal with symmetric phase distribution. The carriers are separated, $\Delta f=50 \mathrm{MHz}$ and the first frequency is $f_{1}=10.9 \mathrm{GHz}$. The gap is set to $0.6 \mathrm{~mm}$, and the material is ECSS silver [EST03], being the multipactor order of 5.

\section{Figure 5.20.}

Example of voltage breakdown vs phase for 6 carrier signal with symmetric phase distribution. The carriers are separated, $\Delta f=50$ $\mathrm{MHz}$ and the first frequency is $f_{1}=10.9$ $\mathrm{GHz}$. The gap is set to $2 \mathrm{~mm}$, and the material is ECSS silver [EST03], being the multipactor order of 15 .

separated, $\Delta f=50 \mathrm{MHz}$ and the first frequency is $f_{1}=11.06 \mathrm{GHz}$. The gap is set to $0.6 \mathrm{~mm}$, and the material is ECSS silver [EST03], being the multipactor order of 5. Fig 5.18 represents the breakdown voltage as a function of the phase. In this case, two local minima and two maxima can be observed $\left(\varphi=0^{\circ}\right.$ and $\varphi=360^{\circ}$ is the same phase). Actually, the global minimum corresponds to $\varphi=0^{\circ}$, but 
there is not significative difference with $\varphi=180^{\circ}$.

The second case, represented in Fig. 5.19, shows a 6 carrier signal with symmetric phase distribution, yielding two degrees of freedom for the phases, $\varphi_{1}$ and $\varphi_{2}$. The carriers are separated, $\Delta f=50 \mathrm{MHz}$ and the first frequency is $f_{1}=10.9 \mathrm{GHz}$. Again, the gap is set to 0.6 $\mathrm{mm}$, and the material is ECSS silver [EST03], being the multipactor order of 5. The contour plot of Fig. 5.19 shows combinations of $\varphi_{1}$ and $\varphi_{2}$ which yields higher voltage breakdown than others. The global minima are marked with a blue cross and correspond to the in-phase case.

Finally, the third case is shown in Fig. 5.20. Again, a 6 carrier signal with symmetric phase distribution with carriers separated, $\Delta f=50 \mathrm{MHz}$, the first frequency being $f_{1}=10.9 \mathrm{GHz}$. The coating material in this case is also silver, but the gap has been set to $2 \mathrm{~mm}$, yielding a multipactor nominal order of 15 (see for example [EST03]). As in the other cases a symmetric pattern is observed and two local minima are found (and marked with blue crosses). With respect to the second case, the minima are now shifted to non-zero values, yielding in a wider envelope than in the in-phase case. In addition, local minima appear dispersed along the whole domain.

From these three particular cases, two interesting conclusions may be extracted and extrapolated to the general case. First, in all cases more than one global minimum has been found. In addition, local minima may also appear, which renders the optimization process hard in order to be robust (it could get stuck in a local minima yielding a wrong solution). Finally, as already discussed within the 20-gapcrossing rule section (Section 4.5.2.2), for the same SEY function, it seems that low multipactor orders tend to in-phase distributions, whereas larger orders tend to spread the envelope. Of course, this should also depend on the particular SEY of the considered material.

\subsection{Discussion on experimental results}

In this Section, the numerical predictions and experimental results of $\left[\mathrm{AVG}^{+} 12\right]$ (Section 4.5) are revisited, giving some aditional discussion with respect to the original paper.

Concretely a more detailed discussion on the results affecting the 
20-gap-crossing rule is given, since they may be valuable to verify or discard some of the historically accepted assumptions about this rule with little or no justification.

Fig. 5.21 represents a summary of the prediction and experimental results published in $\left[\mathrm{AVG}^{+} 12\right]$ (Section 4.5). It is repeated here for completeness.

As already mentioned in the original publication, the breakdown power level for the samples with large gaps resulted in higher values than those predicted by the prediction methods, in part, due to the known increase of the multipactor breakdown for high multipactor orders in waveguides $\left[\mathrm{SRA}^{+} 07\right]$. This implied that for many of the large gap samples the available test power was not enough for starting the multipactor discharge. Therefore, there are many more data for short gaps (multipactor order 1) than for large gaps.

Keep in mind that there are two dimensions for assessing the accuracy of the prediction methods. All prediction methods give, on the one hand, the lowest breakdown (amplitude) power per carrier, but since the breakdown power depends on the phase combination of the carriers, this value is related to a set of phase values for the carriers. This combination of phases, which renders the lowest breakdown power per carrier, is called the worst-case.

In the design process, only the lowest breakdown power is relevant. The combination of phases of the worst-case is not of much importance, since the phases are not under control and shift randomly during operation. Therefore, one way to assess the accuracy of any prediction method is to pay attention only to the predicted breakdown power. This is called "global error" in $\left[\mathrm{AVG}^{+} 12\right]$.

However, when testing in laboratory, it is important to know what are the worst-case phases in order to detect the multipactor discharge to the lowest possible value. According to TESAT Spacecom experience, and also verified during the activity, leaving the phases unlocked, in what is called free-running, make them to shift randomly during the testing in such a way that usually the worst-case combination is found. This may be a useful technique but, since the test time is limited, it is not guaranteed that the free-running mode finds the worst-case, and it is not guaranteed that a local minimum has not been found instead. Therefore it is desirable to use, in addition to the free-running case, an estimation of the worst-case phases coming 
Figure 5.21.

Summary of breakdown threshold predictions and test results for $\mathrm{Ku}$-band samples. FEST3D simulations are labeled with "Num" tag. Labels "c" and "n" stand for contiguous and non-contiguous frequency schemes. Breakdown levels are given in power per carrier. The minimum breakdown power of all tested phase configurations has been plotted with label "Meas". The maximum applied power for the cases where no multipactor has been observed has been also included with label "Meas"".
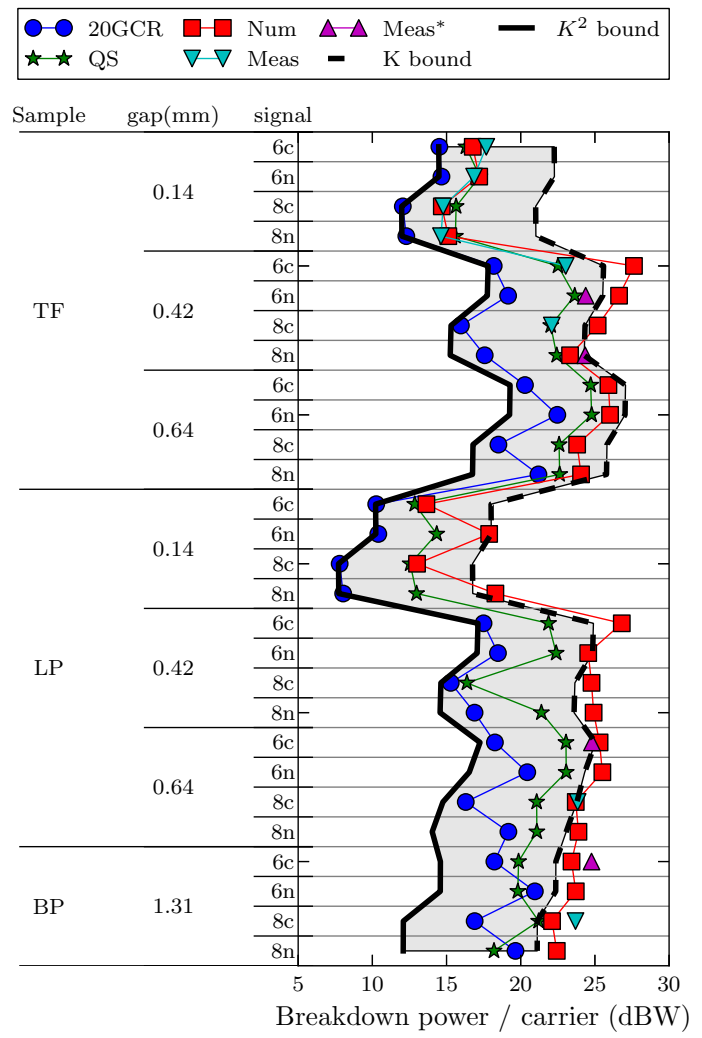

out from a prediction in order to have more control of the testing. This is where the second dimension of accuracy assessment come into play. The "local error", described in $\left[\mathrm{AVG}^{+} 12\right]$, is defined as the error between the predicted breakdown level and the measured one, where the worst-case phase combination, predicted with the same method, has been used. In other words, the "local error" measures the accuracy of the predictor for finding consistent phases and amplitudes. A good prediction method must keep both global and local errors low.

Let us focus on the global error. In Fig. 5.21, it can be seen that the 20-gap-crossing rule is, in general, very conservative. However, it has not a constant behaviour. According to these results, short gaps present a higher margin than large ones (predictions closer 


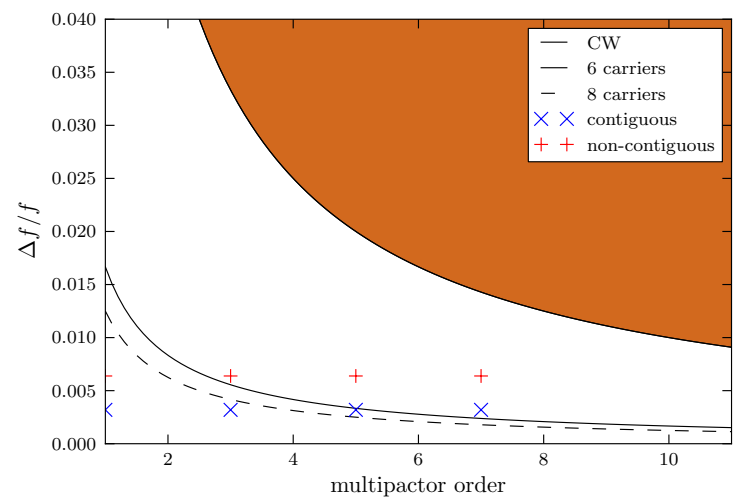

Figure 5.22.

$\mathrm{Ku}$-band samples on 20-gap crossing $T_{20} / T_{e}$ map.

to $K$-bound), and contiguous frequency schemes also present higher margins as compared to non-contiguous ones.

This can be justified with the study of the 20-gap-crossing rule done in 5.2.2. The 20-gap-crossing rule can give predictions which are closer to the $K$-bound, in-phase schemes, or to the $K^{2}$-bound, continuous-wave schemes, depending only on the multipactor order and the carrier separation.

When plotting the samples of $\left[\mathrm{AVG}^{+} 12\right]$ in a $T_{20} / T_{e}$ map, see Fig. 5.22 , the short gap samples and contiguous schemes lay closer to the in-phase case than the others, justifying the experimental findings.

This is important because it is thought by a large portion of the community that the 20 -gap-crossing rule is always conservative. However, these results show that it does not have always the same degree of conservativeness, opening the possibility that it may not be conservative in some cases. For example, it could be expected that the 20-gap-crossing rule predictions are not conservative when they lay close to the CW curve in Fig. 5.22.

With regard to the "local error", the 20-gap-crossing rule shows large discrepancies between prediction and testing. There is a big difference between the predicted breakdown power and the tested breakdown power in laboratory. For example, in most of the samples, the 20-gap-crossing rule predictions show unrealistic low multipactor breakdown power levels, compared to the corresponding test results (see Fig. 5.21). In Table 4.20, it can be seen that, in the test for 
the $0.42 \mathrm{~mm}$ gap transformer and 8 carrier contiguous scheme signal, there was a multipactor discharge with both FR and QS phases but not for the 20-gap-crossing rule phases.

Summarizing, the 20-gap-crossing rule is not precise in both global and local errors, compared with the Quasi-stationary predictions (see Table 4.21 of Section 4.5). In addition, the 20-gap-crossing rule can lead to very misleading conclusions. In the design phase, unrealistically low 20-gap-crossing rule predictions imply very restrictive constraints, but in the test phase, the 20-gap-crossing rule phases are not conservative, yielding a higher breakdown power than the real threshold, and thus falsely validating components. 


\section{Chapter 6}

\section{Conclusions and future work}

The motivation of this thesis originates from the need of having a reliable prediction method for multipactor discharge in multi-carrier systems. At the beginning of this work, not much theory was available for multi-carrier signals. Of course, up to now, the experience of the industry in this area has been very valuable for the correct multipactor-free design. Unfortunately, such a knowledge is usually kept as a company competitive advantage and it is not commonly shared with the community. On the other hand, the existing multipactor design rules for multi-carrier devices proposed in some studies, such as the well-known 20-gap-crossing rule, assume very simplistic scenarios and are totally based on single-carrier theories. This lack of knowledge on the multipactor phenomenon for the multi-carrier case implies significant uncertainties and prediction errors which are compensated by large design margins.

Hence, although the final objective of this thesis has been to provide a more sophisticated prediction method, the studies done throughout this work have been quite heterogeneous, forced to cover the aforementioned unknown areas of the phenomenon. 


\subsection{Conclusions}

The first, and probably most important, unresolved issue in the multipactor theory is the establishment of a discharge criterion based on the number of electrons or charge density in the device.

In practical situations, multipactor is unacceptable when produces some detectable effects on the system, such as noise, reflected power, heating, etc. It is evident that only few electrons in the discharge have negligible impact in the normal functioning of most equipments. This poses a fundamental and unanswered question: how large the electron density should be in order to produce a detectable (or annoying) discharge.

Traditionally, this has been of little importance in single-carrier operation, since, once the resonance conditions are met, the electron number increases indefinitely until a saturation regime is reached. However, the electron density in the multi-carrier case oscillates with a saw-tooth like shape, following the periodical oscillations of the multi-carrier envelope. Until now, there is not a clear criterion that establishes the level that the electronic density needs to reach in order to produce a discharge.

The 20-gap-crossing rule was the first attempt to give an answer to this question, establishing a minimum number of consecutive electron impacts, within one period of the envelope, in order to develop the discharge. However this rule is based upon a very limited set of tests and simulations and is very simplistic, since it disregards fundamental aspects of the phenomenon such as the SEY properties of the material or electron impact energy, proposing a universal rule for all cases.

This work proposes an alternative discharge criterion. Instead of focusing on single periods ${ }^{1}$ of the multicarrier envelope, for which a criterion based on electron density is needed, we have searched situations in which there is always an increasing number of electrons, such as in the single-carrier case. This is the long-term discharge covered in Chapter 4.1, which establishes the conditions by which the

${ }^{1}$ Known as singleperiod or singleevent discharges. electron population accumulates period-after-period of the envelope. It is unknown, by the moment of speaking, which of the two criteria, single-period or long-term, is the most restrictive for all cases. Although, logically, it should depend on each particular case. However, this will be answered only when the discharge criterion based 
on charge density is ever solved. Nevertheless, the single-period discharges are supposed to be very short and weak in time and therefore is more likely that all detections in laboratory are due to long-term discharges.

Despite all this, the novel long-term discharge criterion proposed in this Thesis allows, for the first time, for the rigorous study of multipactor in multi-carrier systems and it is the key for constructing a reliable breakdown prediction method.

On top of this, regarding the development of a new multi-carrier theory, it was necessary to develop a model which incorporates both processes of electron emission and absorption ${ }^{1}$. The classical theory and derivatives, were not valid, in first place, because they just find resonant conditions for the equations of motion. This implies that the classical theory is able to find the breakdown level, and to model electron growth above it, but it is not able to model absorption. In second place, the classical theory is known to be not precise for high multipactor orders, mainly because it assumes a fixed deterministic electron emission velocity, whilst the secondary electron emission is random by nature. For this reason, the existing statistical theories turned out to be a good alternative, since they incorporate the random behaviour of the electron emission velocity and thus are able to accurately modeling the multipactor process for all resonant orders. However, the statistical theories imposes a stationary regime, solving a Fredholm equation, which assumes a family of electrons to multiply and survive to all others. This fact renders this kind of theories also incompatible for modeling situations in which there is an overall electron absorption.

The main theoretical contribution of this thesis is the new nonstationary theory which has been developed in order to cover the aforementioned needs, i.e. to incorporate random electron emission and to cover both electron growth and absorption. The nonstationary theory uses the statistical theory as a basis, but formulates another kind of problem which is not stationary anymore but time-dependent. This implies solving a system of Volterra integral equations which models accurately the electron number evolution in time, including growth and absorption, above and below the breakdown level.

The non-stationary theory was formulated originally for single- 
${ }^{1}$ Also known as worst case

\footnotetext{
${ }^{2}$ In conjunction with the longterm discharge criterion

${ }^{3}$ For average desktop PCs at the time of writing this Thesis, 2013.
}

carrier signals although extended later to the multi-carrier case. The non-stationary theory for multi-carrier signals fully models the electron evolution for any number of carriers and arbitrary combinations of amplitudes and phases per each carrier. This theory constitutes the first full theory of multipactor for multi-carrier signals, at least to the author's knowledge.

Although having developed a completely new theory for multicarrier signals, it is not enough to constitute a prediction method. A breakdown prediction method for multi-carrier signals has to provide not only a threshold amplitude per carrier (as the single-carrier case), but also has to provide which combination of phases yields the lowest breakdown among all. In this thesis we propose a predictor that uses a global optimization algorithm to find the minimum breakdown level among all combination of phases ${ }^{1}$.

One practical issue in order to develop a prediction method is that many phase combinations have to be solved, a number that depends exponentially with the number of carriers. In principle, for each step of the optimizer, the full multi-carrier non-stationary theory can be used to compute the breakdown level ${ }^{2}$. However, there is not a general closed solution of the analytical equations posed by the nonstationary theory, and therefore, those have to be solved numerically, with a non-negligible computational cost. For the current available CPU resources ${ }^{3}$, it was impractical to perform breakdown prediction through brute-force global optimizers using the full multi-carrier non-stationary theory. The time for solving the involved equations, hundred of thousand or even million times, was estimated in weeks or months.

For this reason, an alternative for estimating the electron time evolution in multi-carrier signals has been proposed, which is the quasi-stationary method. This method assumes that the multi-carrier envelope has a very slow time variation compared to the RF signal period. Thus, at each particular time, the electrons will be created or absorbed according to the level of the multi-carrier envelope, using single-carrier approximation. In practice, this means that the non-stationary equations must be solved only once, at the beginning of the optimization, and for different signal amplitudes. Then, with simple approximations, the electron evolution for any multi-carrier envelope waveform can be approximated with little computational 
cost. This makes this method very suitable for massive global optimizer computations.

Based on this quasi-stationary method, together with a genetic global optimizer, a multipactor prediction method for multi-carrier signals has been developed in this thesis. This method is the first one able to find the worst case ${ }^{1}$, by using the long-term multipactor criterion. It is valid for any multicarrier signal with arbitrary number of carriers.

The experimental tests conducted on Ku-band samples during this research activity show a good agreement with the quasi-stationary method with better prediction accuracy than the existing 20-gapcrossing rule. The latter yields conservative-in-excess predictions, at least for this specific kind of samples and multi-carrier signals.

Indeed, the 20-gap-crossing rule has been widely used in the design standards for multi-carrier operation for the last years, because it is thought to be conservative in all cases. However the outcome of this Thesis reveals many exceptions of this rule that would imply that it is not generally valid for multipactor design and testing purposes.

It would be easy to overcome this 20-gap-crossing rule non conservativity issue by specifying extra design margins to the currently applied ones. However, this would make the prediction rule restrictive in excess, with the consequent implications to the industry, regarding component design cost, volume and weight. The selection of a more precise and versatile prediction method, such as the quasi-stationary method developed in this Thesis, ensures conservativity with lower design margins.

\subsection{Future work}

The non-stationary multipactor theory for multi-carrier signals, as well as the quasi-stationary method, are the first theoretical tools able to fully model the complex electron dynamics under multi-carrier signals. Moreover, the experimental tests presented in this Thesis are one of the most complete experimental works with multi-carrier signals ever performed.

However, there is still a long road ahead and many unknown issues. In first place, and as mentioned above, the long-term discharge 
criterion has been used, but little is known about single-period discharges and the connection with the electronic density increase and the effect on the system.

Steps to find the relationship between the electron density and the discharge seem to be in the direction of modelling the adverse effects of the multipactor discharge, such as noise or harmonics. If we are able to have a direct equation relating electron density and the level of the produced disturbances, it is straight-forward to define a criterion based on certain design specifications for maximum allowed noise, or other similar restriction. Some works towards this goal have been already done. For instance, in Chapter 4.2 numerical simulations with PIC code obtain the multipactor radiation spectrum of certain structures. Other way is by means of theoretical modeling of the field emitted by an electron current on some kind of simplified geometry, such as the work presented in [GSA ${ }^{+}$09]. Unfortunately, no definitive conclusions can be extracted yet and more work is needed in order to have a universal electron density criterion.

Future research on this topic is very interesting and valuable for the predictions since they will shed more light to the subject. In the case that a new criterion is found, the multi-carrier non-stationary theory and the quasi-stationary prediction technique can be easily adapted since, in essence, both model electron grow and absorption with time. Thus, it would be easy to change the criterion from electron accumulation to any electron maximum threshold.

On the other hand, the presented theory is based on one dimensional parallel plate geometries, which is valid for rectangular waveguides or other components with relatively short gaps and relatively uniform fields. It can be used as a reference, since it represents the worst-case. Indeed, more complex geometries with non-uniform fields introduce extra electron loss mechanisms that would rise the breakdown level compared to the parallel-plate case. However, the extension of the non-stationary theory to more complex 3D-geometries and different signal modulations is interesting in order to be more precise for those particular cases. Suitable geometries for such an extension could be coaxial, circular and rectangular waveguides. Modulation schemes such as BPSK or QAM would be candidates for their study. Already, some work on these areas has been published for other multipactor theories and could be used as a reference. 
Regarding the prediction, the quasi-stationary method seemed to be more precise for low $f \times d$ products $^{1}$, even though there were not many experimental data on large gaps due to the test-bench limitations. Evidently, the quasi-stationary method is a fast approximation. Predictions with full multi-carrier theory would, in principle, yield better results. The unstoppable technology evolution will bring more and more powerful CPUs which will make possible in the future to use, in practical situations, global optimization with nonstationary multi-carrier theory. However, it is interesting to improve the computational efficiency of the numerical solution of the statistical TTPD functions and Volterra integral equations. This would lead to a faster adoption of the full multi-carrier theory and probably improve the predictions for larger $f \times d$ products. For this reason, more experimental tests for larger gaps would be also desirable in order to contrast the theoretical predictions. In addition, experimental tests on other frequency bands and with other technologies would help to assess the prediction validity for a wider range of situations.

Little was known at the beginning of this study about the multipactor phenomenon in multi-carrier signals. All the efforts in this thesis have been devoted to provide a better basic understanding of all physical processes involved in the phenomenon, as well as in the development of a completely new theoretical framework, in order to fully characterize the electron dynamics under such complex signals. As a result, a new theory and a prediction method have been proposed. Despite their current limitations, an important step forward has been taken and, hopefully, it will be the beginning of more extensive studies on this matter. 


\section{Appendix A}

\section{Electron Seeding Techniques}

Even if the conditions to initiate multipactor are met, there must be an initial population of electrons inside the waveguide in order to set-up a discharge. The number of such seeding electrons and their energy determine the probability of a multipactor discharge to take place.

In the space environment, the source of seeding electrons is the particle radiation due to the trapped environment in the magnetosphere, Solar Flares or Cosmic Rays. The total flux of electrons reaching the interior of the device depends on the satellite geometry, materials and orbit.

On the other hand, in laboratory multipactor tests, electron seeding is necessary to obtain significant results [WJ89, GW96, RW95]. The purpose of this section is to model, and give an estimation, of the initial population and energy distribution of the electron seeding for three kind of sources: radioactive sources, Ultra-Violet (UV) lamps and Regulated Electron Gun (REG).

For the radioactive sources, the electrons can be generated in the interior of the device in two ways. Either the impacting electrons 
may have enough energy to go through the metallic device walls, or secondary electrons are generated inside the device wall as the impacting electron penetrates and looses energy. Both processes may occur at the same time. Similarly, in the space environment, impacting protons and ions can also produce secondary electrons inside the component.

\section{A.1 Radioactive sources}

A radioactive source consists of a certain amount of radioactive material permanently sealed in a capsule, or closely bonded in an inert substance. The radioactive material emits electrons or beta-rays, by means of beta-decay, in which a parent nucleus, with $Z$ protons, becomes a daughter one, with $Z+1$ protons, when one of the neutrons turns into a a proton. In order to comply with the principle of conservation of charge, an electron is released along with a neutrino, which is electrically neutral and hence, ignored for the present purpose. The disintegration scheme is

$$
n \rightarrow p^{+}+e^{-}+v
$$

where $n, p^{+}, e^{-}$, and $v$ represent the parent neutron, the daughter proton, the electron and the neutrino, respectively.

The radioactive source is placed outside the waveguide, close to the critical point in which multipactor takes place. Therefore, the main advantage is that it is a non-invasive method and the electrons act directly on the critical point in the waveguide, provided that their energy is high enough to go through the external metallic walls of the waveguide and penetrate to its interior.

Radioactive sources have different shapes and sizes depending on their application. A typical source of such kind, commonly used in multipactor tests, is based on strontium-90 (Sr90) thanks to its relatively simple decay chain. The $\mathrm{Sr} 90$ is a radioactive isotope of $\mathrm{Sr}$ which is an almost perfect beta-emitter (electron emitter). It experiences a beta-decay, with half life of 28.8 years, to yttrium-90 (Y90), also unstable with a half life of 64 hours, which in turn undergoes beta decay to the stable zirconium-90 (Z90). The characteristics of both electron emissions are summarized in table A.1. 
Figure A.1.

Beta-decay chain for SR90.

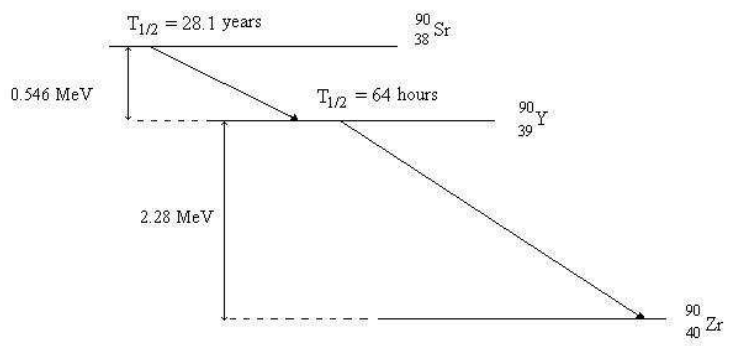

Table A.1. Strontium and yttrium beta radioactivity characteristics.

\begin{tabular}{|c|c|c|c|c|c|}
\hline Source & half-life & $\begin{array}{c}\text { decay } \\
\text { constant } \\
\left(\mathrm{s}^{-1}\right)\end{array}$ & $\begin{array}{c}\text { Specific } \\
\text { activity } \\
(\mathrm{Bq} / \mathrm{g})\end{array}$ & $\begin{array}{c}\text { Mean } \\
\text { energy } \\
(\mathrm{KeV})\end{array}$ & $\begin{array}{c}\text { Max } \\
\text { Energy } \\
(\mathrm{KeV})\end{array}$ \\
\hline Sr90 & $9.08 \times 10^{8}$ & $7.6 \times 10^{-10}$ & $5.18 \times 10^{12}$ & 195.8 & 546 \\
\hline Y90 & $2.30 \times 10^{5}$ & $3 \times 10^{-6}$ & $2.04 \times 10^{16}$ & 937 & 2280 \\
\hline
\end{tabular}

For any beta-decay process, in order to find the expression for the maximum kinetic energy of the emitted electrons, let us consider the parent nucleus at rest which disintegrates into a daughter nucleus and an electron (the influence of the neutrino can be neglected).

The electron obtains its maximum kinetic energy when the daughter nucleus comes to rest. Due to conservation of mass-energy, this implies an electron maximum kinetic energy given by

$$
E_{\text {max }}=\left(m_{P}-m_{D}-m_{e}\right) c^{2},
$$

where the masses of the parent nucleus, $m_{P}$, daughter nucleus, $m_{D}$, and the electron, $m_{e}$ are known; $c$ is the speed of light in free space. Notice that either from (A.2) and Table A.1, the maximum emission energy for Y90 is higher than for Sr90.

On the other hand, the energy spectrum of the emitted electrons, $d \Upsilon / d E$ given in $\mathrm{Bqg}^{-1} \mathrm{eV}^{-1}{ }^{1}$ can be calculated with a modification of the formula given in [Gri87], where the original equation has been
${ }^{1}$ One becquerel, $\mathrm{Bq}$, is defined as the activity of a quantity of radioactive material in which one nucleus decays per second. Its units are equivalent to $\mathrm{s}^{-1}$.

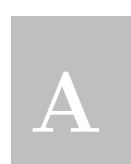


scaled in order to meet with the maximum kinetic energy of (A.2)

$$
\frac{d \Upsilon}{d E}=K E \sqrt{E^{2}-m_{e}^{2} c^{4}}\left[\left(m_{P}-m_{D}\right) c^{2}-E\right]^{2} .
$$

No Coulomb correction factors have been considered. Here, $K$ is the normalization constant which can be numerically calculated as

$$
K=\frac{A_{0}}{\int_{-\infty}^{\infty} d \Upsilon / d E},
$$

where $A_{0}$ is the specific activity of the sample.

A Sr90 source emits a composite Sr90-Y90 radiation. Therefore its energy spectrum is the superposition of pure Sr90 and Y90 spectra with same activity $A_{0}$, which implies an effective activity of $2 A_{0}$. The spectrum of a Sr90 source has been calculated using (A.3) and checked with the experimental results of Sokolov [SPLL03], who measures the electron count in $300 \mathrm{~s}$ for a $25 \mathrm{~Bq}, \mathrm{Sr} 90$ source. Both graphs are presented in Fig. A.2, showing an excellent agreement. Notice the two parts of the spectrum belonging to the contributions of pure Sr90 beta-decay (high peak of lower energy), and Y90 beta-decay (long tail towards high energies).

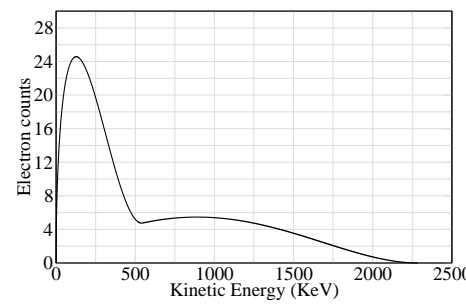

(a) Analytical beta spectrum.

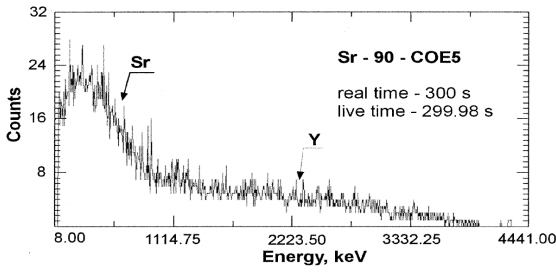

(b) Experimental beta spectrum.

Figure A.2. Electron count for a $25 \mathrm{~Bq}$ SR90 source during $300 \mathrm{~s}$. (a) Analytical results calculated with (A.3). (b) Experimental results extracted from [SPLL03].

There are two ways in which electrons can be produced inside the waveguide by the radioactive source. Either the primary electrons themselves have enough energy to go through the device wall, or, 
while passing through the material, secondary electrons are liberated in the forward direction and enter the waveguide.

The forward secondary emission has the same nature as the traditional (backward) Secondary Electron Yield SEY $\left[\mathrm{AVM}^{+}\right.$07, Vau89].

For typical waveguide wall widths (in the range of $\mathrm{mm}$ ), the interval of electron impact energies giving a forward SEY higher than one is very small and therefore the fraction of secondary electrons with respect to the primary ones is negligible. Therefore, the primary electrons are considered as the only source of free electrons inside the waveguides. However, for other kind of sources, such as proton or heavy ion beams in space environment conditions, forward SEY may become the mean mechanism of electron production and should not be ignored.

On the other hand, the range of an electron with energy $E_{0}$ in a material is defined as the maximum distance that it can travel in straight line before it completely stops. It can be expressed as a function of $E_{0}$ as

$$
R\left(E_{0}\right)=\int_{0}^{E_{0}} \frac{1}{S(E)} d E
$$

where $S(E)$ is the linear stopping power of the material. Fig. A.3 shows the range for aluminium, calculated with (A.5)

Figure A.3.

Range for aluminium vs electron impact energy.

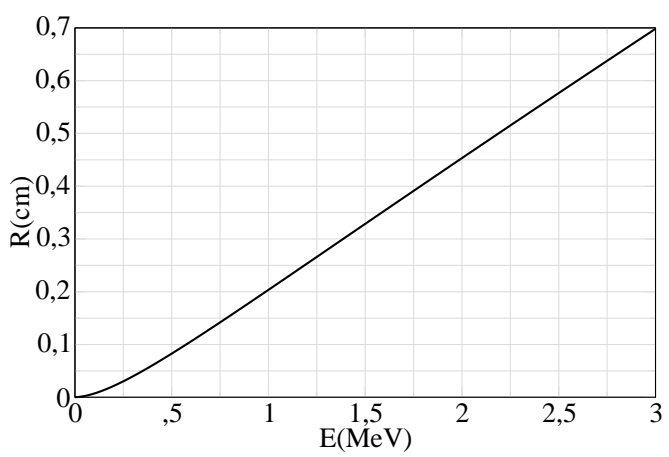

Therefore, if a radioactive source is applied to a metal foil of width $w$, only the electrons with energy $E>E_{\min }$, being $R\left(E_{\min }\right)=w$, penetrate into the device, losing an energy equal to $E_{\text {min }}$. 
Let us study a particular case of a Sr90 source of $37 \mathrm{MBq}$, applied to an aluminium foil with different values of $w$. Firstly, the flux that arrives to the external part of the device wall is $37 \times 2 / 6=12.33 \mathrm{MBq}$. The factor 2 corresponds to the double emission of Sr90 combined with Y90, whereas the factor 6 comes from the assumption of isotropic radiation. The energy spectrum of this flux is the combination of (A.3) for Sr90 and Y90.

Then, the fraction of the flux that pass through the device wall, corresponds to the integration of its energy spectrum for $E>E_{\min }$, having an energy distribution with a maximum of $E_{\max }-E_{\min }$. Table A.2 shows the values of $E_{\text {min }}$, free electron production rate and $E_{\max }-E_{\min }$, for different aluminium widths.

Table A.2. Results of electron production inside the waveguide for a $\mathrm{Sr} 90,37 \mathrm{MBq}$ source in aluminium of different widths.

\begin{tabular}{|c|c|c|c|}
\hline Width $(\mathrm{mm})$ & $E_{\min }(\mathrm{MeV})$ & $\begin{array}{c}\text { Elec. prod. } \\
\text { rate }(\text { elec. } / \mathrm{s})\end{array}$ & $\begin{array}{c}E_{\max }-E_{\min } \\
(\mathrm{MeV})\end{array}$ \\
\hline 0.5 & .33 & $6.17 \times 10^{6}$ & 1.95 \\
\hline 1 & .58 & $4.7 \times 10^{6}$ & 1.7 \\
\hline 4 & 1.8 & $2.5 \times 10^{5}$ & 0.48 \\
\hline 6 & 2.63 & 0 & - \\
\hline
\end{tabular}

Of course, the electron production rate is proportional to the activity of the radioactive source. The spectrum energy of the electrons inside the waveguide is that of Fig. A.2 but shifted $E_{\min }$ to the left. Fig. A.4 below shows an example for a $1 \mathrm{~mm}$ width aluminium foil and the same conditions as Fig. A.2.

\section{A.2 UV lamps}

This kind of sources extract electrons from the inner surfaces of the waveguide by exposing them to an UV light beam, the electrons being produced by means of the photoelectric effect. The UV light is guided from an UV lamp, placed outside of the vacuum chamber, to the interior of the waveguide through an optical fiber. Thus, the basic system consists of two lengths of fused quartz optical fiber, a 
Figure A.4.

Energy spectrum of electrons inside the waveguide for $1 \mathrm{~mm}$ aluminium foil.

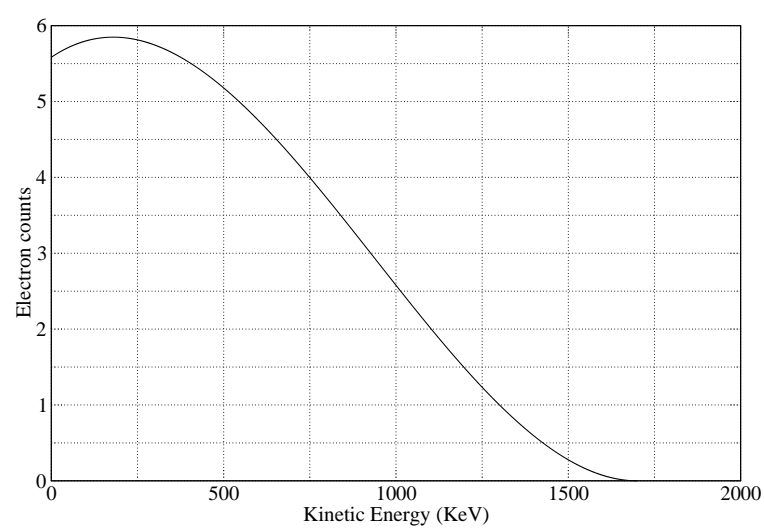

quartz window to access the vacuum chamber maintaining the vacuum environment, and a slot or hole performed in the DUT to allow the fiber to penetrate directly close to the multipactor critical point.

Typically, the electron production rates are significantly higher than for other sources such as radioactive ones and, moreover, the emitted electrons have more suitable energies to produce multipactor. In addition, the intensity of the lamp (which is directly related with the electron production rate) as well as the light incidence angle can be controlled independently, which allows to establishing a much more controlled scenario. The main drawback of these sources is that they are invasive (the DUT geometry must be altered).

According to the photoelectric effect, the energy of the impacting photon, $h \nu$ (where $h$ is the Planck constant and $\nu$ is the photon frequency), must be always greater than the material working function, $\Phi$, in order to produce electrons. Table A.3 shows the value of $\Phi$ for some common materials.

Table A.3.

Work function value for some common metals.

\begin{tabular}{|c|c|}
\hline Metal & $\Phi(\mathrm{eV})$ \\
\hline Silver & 4.26 \\
\hline Copper & 4.5 \\
\hline Gold & 5.1 \\
\hline
\end{tabular}

When UV light of single wavelength impinges on the internal walls of the guide in vacuum conditions, photoelectrons are generated with 
a kinetic energy comprised between 0 and $h \nu-\Phi$.

On the other hand, the number of released photoelectrons per incident photon is given by the absolute yield, $\gamma$, which can be approximated, for low photon energies and UV intensity, using the Fowler's law [Car56], which is valid for metal temperatures much lower than Fermi's temperature $\left(T_{F} \approx 10^{4} \mathrm{~K}\right)$.

$$
\gamma=\frac{N_{e}}{N_{p}}=C(h \nu-\Phi)^{m},
$$

where $N_{e}$ is the quantity of generated photoelectrons, $N_{p}$ is the number of impacting photons, and $C$ and $m$ are material dependent parameters. For the majority of metals, $m \approx 2$.

Experimental values of $\gamma$ for copper and gold have been extracted from Krolikowski results in [KS69] and [KS70] respectively, and used to fit (A.6) and extract the values of $C$ and $m$ for each case. These are presented in Table A.4. Fig. A.5 shows the experimental values and the fitted curves using (A.6).

\begin{tabular}{|c|c|c|c|}
\hline Metal & $\Phi(\mathrm{eV})$ & $C\left(\mathrm{eV}^{-m}\right)$ & $m$ \\
\hline Copper & 4.5 & $4.8 \times 10^{-4}$ & 1.51 \\
\hline Gold & 5.1 & $9.44 \times 10^{-5}$ & 2.82 \\
\hline
\end{tabular}

\section{Table A.4.}

Fit of (A.6) from experimental data of [KS69] and $[\mathrm{KS} 70]$, for copper and gold respectively.

Among all possibilities, the low pressure mercury vapor lamp, is one of the most suitable for multipactor purposes, thanks to its narrow emission spectral density, with a wavelength around $254 \mathrm{~nm}$, and high efficiency of $90 \%$ [RW95]. For the specific case presented by Raboso in [RW95], in which this kind of lamp emitting with a power of $37 \mu \mathrm{W}$ impinges on a copper surface -any lamp, emitting at a power $P$, would generate a photon flux of $P /(h \nu)$ (photons/s)-, the theoretical flux of photoelectrons would be, using (A.6) of $5.7 \times 10^{9}$ electrons/s. This value is similar to the theoretical and considerably higher than the experimental results of Raboso. This is due to the difference in the copper used by Krolikowski, ultra clean copper, and by Raboso, oxidized one. Notice that the production rate for this specific UV source is three order of magnitude higher than that 

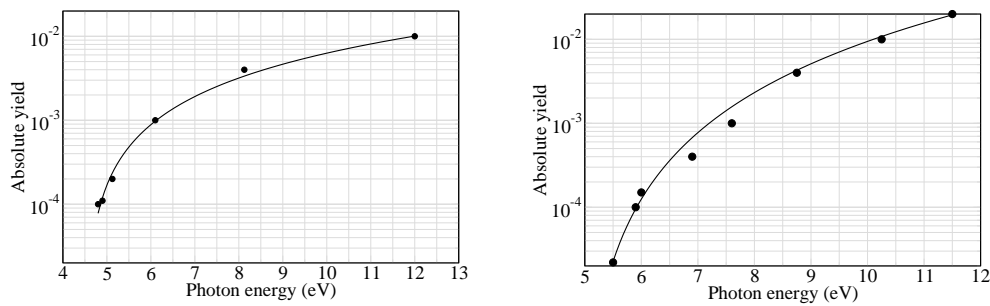

(a) Fitting of Fowler's formula for Copper.

(b) Fitting of Fowler's formula for Gold.

Figure A.5. Fitting of Fowler's formula for Copper and Gold from experimental values. (a) for Copper. (b) for Gold. Experimental values have been extracted from [KS69] and [KS70], for copper and gold respectively.

of the section A.1 for a radioactive source. The production rate is proportional to the lamp power.

\section{A.3 Regulated Electron Gun}

A regulated electron gun (REG) is a mechanism that creates a controlled electron beam with the desired energy and flux (according to the nominal power). It consists of a tungsten filament, a Wehnelt cylinder, electrostatic lenses, an iris and an anode. The current flows through a tungsten filament, the wire starts annealing and electrons are emitted. A certain potential is applied to the Wehnelt cylinder and the emitted electrons form a cloud. This cloud ensures a stable electron beam. Using this device, it is possible to select the energy of the individual electrons (from 20 to $1000 \mathrm{eV}$ ) and the electron density inside the gap.

A beam of intensity $I$ and potential $E$ has a power of $P=I E$. Therefore, for a REG of nominal power $P_{n}$ the flux and energy of the electrons are inversely related as

$$
E=\frac{P_{n}}{I}, \quad I=\frac{P_{n}}{E}
$$


For example for a $20 \mathrm{~mW}$ REG and a potential fixed to $1 \mathrm{KeV}$, the flux of seeding electrons is of $1.25 \times 10^{14}$ electrons $/ \mathrm{s}$.

The REG has the advantage of generating a controled beam, whose energy can be set to the optimum value to initiate a multipactor discharge. The flux of injected electrons is the highest of the three revised methods, but the disadvantage is that the mechanism is bulky and it usually cannot access the critical region of multipactor inside the structure. In waveguides, for example, it must be placed at the input port and the injected electrons must reach the critical gap. Depending on the structure, a big fraction of the injected electrons may not reach it. It is also necessary a direct line of sight from the REG to the critical gap. Therefore it may not be used in all type of DUTs. 


\section{Appendix B}

\section{Demonstration 1}

This section demonstrates that the instantaneous frequency of a multicarrier signal with uniform frequency spacing, equal phase and equal amplitude for all carriers is constant and equal to the average frequency

$$
F(t)=\frac{1}{2 \pi K} \sum_{i=1}^{K} \omega_{i} .
$$

According to (4.55), $F(t)$ is given by

$$
F(t)=\frac{1}{2 \pi} \frac{d P(t)}{d t}=\frac{1}{2 \pi} \frac{I^{\prime}(t) R(t)-I(t) R^{\prime}(t)}{R^{2}(t)+I^{2}(t)},
$$

where $R(t), I(t)$, are the real and imaginary part of the analytic signal and $R^{\prime}(t), I^{\prime}(t)$ are their time derivatives, respectively. Equations (4.53) and (4.54) detail these terms.

After simple algebra manipulations and some rearrangement, the 
denominator of (B.2) can be rewritten as

$$
\begin{aligned}
& R^{2}(t)+I^{2}(t)= \\
& {\left[\sum_{i=1}^{K} E_{i} \cos \left(\omega_{i} t+\phi_{i}\right)\right]^{2}+\left[\sum_{i=1}^{K} E_{i} \sin \left(\omega_{i} t+\phi_{i}\right)\right]^{2}=} \\
& \sum_{i=1}^{K}\left[E_{i} \cos \left(\omega_{i} t+\phi_{i}\right)\right]^{2}+\sum_{i=1}^{K}\left[E_{i} \sin \left(\omega_{i} t+\phi_{i}\right)\right]^{2}+ \\
& 2 \sum_{i<j} E_{i} E_{j} \cos \left(\omega_{i} t+\phi_{i}\right) \cos \left(\omega_{j} t+\phi_{j}\right)+ \\
& 2 \sum_{i<j} E_{i} E_{j} \sin \left(\omega_{i} t+\phi_{i}\right) \sin \left(\omega_{j} t+\phi_{j}\right) .
\end{aligned}
$$

Using the trigonometric identities

$$
\begin{aligned}
& \cos ^{2} \alpha+\sin ^{2} \alpha=1 \\
& \cos \alpha \cos \beta+\sin \alpha \sin \beta=\cos (\alpha-\beta),
\end{aligned}
$$

(B.3) finally becomes

$$
\begin{aligned}
& R^{2}(t)+I^{2}(t)= \\
& \sum_{i=1}^{K} E_{i}^{2}+2 \sum_{i<j}^{K} E_{i} E_{j} \cos \left[\left(\omega_{i}-\omega_{j}\right) t+\phi_{i}-\phi_{j}\right]
\end{aligned}
$$

The numerator of (B.2) is instead

$$
\begin{aligned}
& I^{\prime}(t) R(t)-I(t) R^{\prime}(t)= \\
& \sum_{i=1}^{K} E_{i} \omega_{i} \cos \left(\omega_{i} t+\phi_{i}\right) \sum_{i=1}^{K} E_{i} \cos \left(\omega_{i} t+\phi_{i}\right)+ \\
& \sum_{i=1}^{K} E_{i} \omega_{i} \sin \left(\omega_{i} t+\phi_{i}\right) \sum_{i=1}^{K} E_{i} \sin \left(\omega_{i} t+\phi_{i}\right) .
\end{aligned}
$$


With some rearrangement, it can be rewritten as

$$
\begin{aligned}
& I^{\prime}(t) R(t)-I(t) R^{\prime}(t)= \\
& \sum_{i=1}^{K} E_{i}^{2} \omega_{i}+\sum_{i<j}^{K} E_{i} E_{j}\left(\omega_{i}+\omega_{j}\right) \cos \left[\left(\omega_{i}-\omega_{j}\right) t+\phi_{i}-\phi_{j}\right] .
\end{aligned}
$$

where the identities (B.4) have been used again.

If we impose uniform frequency spacing with lowest frequency $\omega_{0}$ and separation $\Delta \omega$

$$
\omega_{i}=\omega_{0}+(i-1) \Delta \omega,
$$

and equal phase and equal amplitude for all carriers

$$
E_{i}=E_{0}, \quad \phi_{i}=\phi_{0}, \quad \forall i
$$

both expressions (B.5) and (B.7) become

$$
\begin{aligned}
& R^{2}(t)+I^{2}(t)= \\
& E_{0}^{2}\left\{K+2 \sum_{i<j}^{K} \cos [(i-j) \Delta \omega t]\right\}
\end{aligned}
$$

and

$$
\begin{aligned}
& I^{\prime}(t) R(t)-I(t) R^{\prime}(t)= \\
& E_{0}^{2} \omega_{0}\left\{K+2 \sum_{i<j}^{K} \cos [(i-j) \Delta \omega t]\right\}+ \\
& E_{0}^{2} \Delta \omega\left\{\frac{K(K-1)}{2}+\sum_{i<j}^{K}(i+j-2) \cos [(i-j) \Delta \omega t]\right\},
\end{aligned}
$$

respectively. Note that the summation of arithmetic series is $\sum_{i=1}^{K} a_{i}=$ $K / 2\left(a_{1}+a_{K}\right)$. 
Using (B.10), (B.11), and according to (B.2), the instantaneous frequency becomes

$$
\begin{aligned}
& F(t)=\frac{1}{2 \pi} \cdot \\
& \left\{\begin{array}{c}
\frac{K(K-1)}{2}+\sum_{i<j}^{K}(i+j-2) \cos [(i-j) \Delta \omega t] \\
\omega_{0}+\Delta \omega \frac{K}{K}+2 \sum_{i<j}^{K} \cos [(i-j) \Delta \omega t]
\end{array}\right\}
\end{aligned}
$$

Note that the summatories depend exclusively on the sum and difference of the two iterators. Therefore, the expression above can be further simplified by performing a change of variables such that $k=i-j$ and $l=i+j$. The summatories can be unwrapped to yield

$$
\begin{aligned}
& \sum_{i<j}^{K}(i+j-2) \cos [(i-j) \Delta \omega t]= \\
& 2 \sum_{i=1}^{K-1} \cos (i \Delta \omega) \sum_{l=1}^{K-i} l+i / 2-1
\end{aligned}
$$

and

$$
\sum_{i<j}^{K} \cos [(i-j) \Delta \omega t]=2 \sum_{i=1}^{K-1} \sum_{l=1}^{K-i} \cos (i \Delta \omega)
$$

Considering that

$$
\sum_{l=1}^{K-i} l+i / 2-1=\frac{K-1}{2}(K-i)
$$

and that

$$
\sum_{l=1}^{K-i} 1=K-i
$$


the final expression of the instantaneous frequency yields

$$
F(t)=\frac{1}{2 \pi}\left[\omega_{0}+\Delta \omega \frac{K-1}{2}\right]=\frac{1}{2 \pi K} \sum_{i=1}^{K} \omega_{i} .
$$




\section{Bibliography}

$\left[\mathrm{AAA}^{+} 09\right]$ I. Arregui, S. Anza, I. Arnedo, C. Vicente, A. Lujambio, J. Gil, M. Chudzik, B. Gimeno, T. Lopetegi, MAG Laso, et al. Multipactor prediction in novel high-power low-pass filters with wide rejection band. In Microwave Conference, 2009. EuMC 2009. European, pages 675678. IEEE, 2009.

[ADDM09] J.-C. Angevain, L.S. Drioli, P.S. Delgado, and C. Mangenot. A boundary function for multicarrier multipaction analysis. In Antennas and Propagation, 2009. EuCAP 2009. 3rd European Conference on, pages 2158 -2161, march 2009.

$\left[\mathrm{AMA}^{+} 10\right]$ S. Anza, M. Mattes, J. Armendariz, J. Gil, C. Vicente, B. Gimeno, V.E. Boria, and D. Raboso. RF breakdown prediction for microwave passive components in multi-carrier operation. In Frank Sabath, D.V. Giri, Farhad Rachidi, and Armin Kaelin, editors, UltraWideband, Short Pulse Electromagnetics 9, pages 375381. Springer New York, 2010.

$\left[\mathrm{AMV}^{+} 11\right]$ S. Anza, M. Mattes, C. Vicente, J. Gil, D. Raboso, V. E. Boria, and B. Gimeno. Multipactor theory for multicarrier signals. Physics of Plasmas, 18(3):032105, 2011. 
[AUR11] CSIC-UAM AURORASAT. CCN of Multipactor in multicarrier RF systems. Contract AO/14978/05/NL/GLC. Final Report: SEY database, Secondary Electron Emission Measurements, EDX, SEM and Multipactor Breakdown Simulations. Technical report, AURORASAT, 2011.

$\left[\mathrm{AVG}^{+} 07\right]$ Sergio Anza, Carlos Vicente, Benito Gimeno, Vicente E. Boria, and Jaime Armendariz. Long-term multipactor discharge in multicarrier systems. Physics of Plasmas, 14(8):082112-082112-8, Aug. 2007.

$\left[\mathrm{AVG}^{+} 10\right] \quad$ S. Anza, C. Vicente, J. Gil, V. E. Boria, B. Gimeno, and D. Raboso. Nonstationary statistical theory for multipactor. Physics of Plasmas, 17(6):062110, June 2010 .

$\left[\mathrm{AVG}^{+} 12\right]$ S. Anza, C. Vicente, J. Gil, M. Mattes, D. Wolk, U. Wochner, V.E. Boria, B. Gimeno, and D. Raboso. Prediction of multipactor breakdown for multicarrier applications: The quasi-stationary method. $\mathrm{Mi}$ crowave Theory and Techniques, IEEE Transactions on, 60(7):2093-2105, july 2012.

$\left[\mathrm{AVM}^{+} 07\right] \quad$ S. Anza, C. Vicente, M. Mattes, B. Gimeno, V. Boria, and J. Gil. Multipactor in multicarrier RF systems. Contract AO/1-4978/05/NL/GLC. Technical Note No.1. Technical report, Aurora SAT, 2007.

[AVR $\left.{ }^{+} 08\right]$ Sergio Anza, Carlos Vicente, David Raboso, Jordi Gil, Benito Gimeno, and Vicente E. Boria. Enhanced Prediction of Multipaction Breakdown in Passive Waveguide Components including Space Charge Effects. In IEEE International Microwave Symposium, pages 1095-1098, Atlanta, USA, Jun 2008.

[BL05] C.K Birdsall and A.B. Langdon. Plasma Physics Via Computer Simulation. Taylor \& Francis Group, London, UK, 2005. 
[Bru54] H. Bruining. Physics and Applications of Secondary Electron Emission. McGraw-Hill Book Company, Inc., New York, 1954.

[Car56] Paul E. Carroll. Photoelectric work function from analysis of emission in an accelerating field. Physical Review, 104(3):660-661, Nov 1956.

$\left[\mathrm{CCF}^{+} 04\right]$ R. Cimino, I. R. Collins, M. A. Furman, M. Pivi, F. Ruggiero, G. Rumolo, and F. Zimmermann. Can low-energy electrons affect high-energy physics accelerators? Physical Review Letters, 39(1):(014801)1-4, Jul. 2004 .

[CE74] M. S. Chung and T. E. Everhart. Simple calculation of energy distribution of low-energy secondary electrons emitted from metals under electron bombardment. Journal of Applied Physics, 45(2):707-709, 1974.

[Cho00] E. Chojnacki. Simulations of a multipactor-inhibited waveguide geometry. Physical Review Special Topics Accelerators and Beams, 3(3):(032001)1-5, 2000.

[CMK07] R. J. Cameron, R. Mansour, and C. M. Kudsia. Microwave Filters for Communication Systems: Fundamentals, Design and Applications. John Wiley \& Sons, 2007.

$\left[\mathrm{CMM}^{+} 09\right]$ F. Caspers, E. Montesinos, I. Montero, C. MiquelEspanya, D. Raboso, B. Gimeno, C. Vicente, S. Anza, J. Gil, and L. Conde. A novel technique for mitigating multipactor by means of magnetic surface roughness. Proceedings of PAC09, 4(July):9-11, 2009.

[CST] CST Computer Simulation Technology Center. http://www.cst.de/.

[DEGL08] John H. Drew, Diane L. Evans, Andrew G. Glen, and Lawrence M. Leemis. Transformations of random variables. In Computational Probability: Algorithms and Applications in the Mathematical Sciences, volume 
117 of In Operations Research 83 Management Science, pages 45-54. Springer US, 2008.

$\left[\mathrm{dLPA}^{+} 06\right] \quad J$. de Lara, F. Pérez, M. Alfonseca, L. Galán, I. Montero, E. Román, and D. Raboso. Multipactor prediction for on-board spacecraft RF equipment with the MEST software tool. IEEE Transactions on Plasma Science, 34(2):476-484, Apr. 2006.

[DS05] Amos Dexter and Rebecca Seviour. Rapid generation of multipactor charts by numerical solution of the phase equation. Journal of Physics D: Applied Physics, 38(9):1383, 2005.

[ECS] ECSS. Multipactor Tool. http://www.aurorasat.es/multipactortool.php.

[EST03] Space Engineering: Multipacting Design and Test, volume ECSS-20-01A, edited by ESA-ESTEC. ESA Publication Division, The Netherlands, May 2003.

[Far34] P.T. Farnsworth. Television by electron image scanning. Journal of the Franklin Institute, 218(4):411-444, Oct. 1934.

[FES] FEST3D Full-wave Electromagnetic Simulation Tool. http://www.fest3d.com/.

[FP02] M.A Furman and M.T.F. Pivi. Probabilistic model for the simulation of secondary electron emission. Physical Review Special Topics - Accelerators and beams, 5:(124404)1-18, Dec. 2002.

[Fri00] L. Friederichs. The significance of amplitude and time statistics of multiple carrier signals for multipactor testing. In Proceedings of the 3rd International Workshop on Multipactor, RF and DC Corona and Passive Intermodulation in Space RF Hardware, pages 79-84. ESTEC, Noordwijk, The Netherlands, Sept. 4-6 2000. 
$\left[\mathrm{GGC}^{+}\right.$05] R.L. Geng, P. Goudket, R.G. Carter, S. Belomestnykh, H. Padamsee, and D.M. Dykes. Dynamical aspects of multipacting induced discharge in a rectangular waveguide. Nuclear Instruments and Methods in Physics Research Section A: Accelerators, Spectrometers, Detectors and Associated Equipment, 538(1-3):189 - 205, 2005 .

[Gil92] A. Gilardini. New breakdown modes of the multipacting discharge. Journal of Applied Physics, 9(71):46294631, May 1992.

[Gi195] A. Gilardini. Multipacting discharges: Constant-k theory and simulations results. Journal of Applied Physics, 2(78):783, Jul. 1995.

GIPA $\left.^{+} 12\right]$ D. Gonzalez-Iglesias, A. M. Perez, S. Anza, J. Vague, B. Gimeno, V. E. Boria, D. Raboso, C. Vicente, J. Gil, F. Caspers, and L. Conde. Multipactor in a coaxial line under the presence of an axial dc magnetic field. Electron Device Letters, IEEE, PP(99):1 -3, 2012.

[GISA $\left.{ }^{+} 12\right]$ D. Gonzalez-Iglesias, P. Soto, S. Anza, B. Gimeno, V.E. Boria, C. Vicente, and J. Gil. Multipactor susceptibility charts for ridge and multiridge waveguides. Electron Devices, IEEE Transactions on, 59(12):3601 -3607 , dec. 2012.

$\left[\mathrm{GPB}^{+}\right.$03] R.L Geng, H Padamsee, S Belomestnykh, P Goudket, D.M Dykes, and R.G Carter. Suppression of multipacting in rectangular coupler waveguides. Nuclear Instruments and Methods in Physics Research Section A: Accelerators, Spectrometers, Detectors and Associated Equipment, 508(3):227 - 238, 2003.

[Gre02] J. Greenwood. The correct and incorrect generation of a cosine distribution of scattered particles for MonteCarlo modelling of vacuum systems. Elseviewer Science, Vacuum, 67(2):217-222, Sept. 2002. 
[Gri87] D. J. Griffiths. Introduction to Elementary Particles. John Wiley \& Sons, Inc, 1987.

$\left[\mathrm{GSA}^{+} 09\right]$ B. Gimeno, E. Sorolla, S. Anza, C. Vicente, J. Gil, A. M. Pérez, V. E. Boria, F. J. Pérez-Soler, F. Quesada, A. Álvarez, and D. Raboso. Multipactor radiation analysis within a waveguide region based on a frequencydomain representation of the dynamics of charged particles. Phys. Rev. E, 79(4):046604, Apr 2009.

[GvE48] E. W. B Gill and A. von Engel. Starting potentials of high-frequency gas discharges at low pressure. Proceedings of the Royal Society of London, Series A, Mathematical and Physical Sciences, 192(1030):446-463, Feb. 1948.

[GW96] K. H. Geisser and D. Wolk. Multipactor testing of multiplexer and waveguide components exposed to multiple carrier loading. In Proceedings of the 2nd International Workshop on Multipactor, RF and DC Corona and Passive Intermodulation in Space RF Hardware. ESTEC, Noordwijk, The Netherlands, Mar. 12-13 1996.

[HFS] ANSYS INC. http://www.ansyst.com/.

$\left[\mathrm{HRS}^{+} 11\right] \quad$ J. Hueso, D. Raboso, D. Schmitt, V.E. Boria, B. Martinez, and C. Vicente. Study of the multipactor effect in bandpass wedge-shaped waveguide filters. Electron Devices, IEEE Transactions on, 58(9):3205 -3212, sept. 2011.

[HW54] A.J. Hatch and H.B. Williams. The secondary electron resonance mechanism of low-pressure high-frequency gas breakdown. Journal of Applied Physics, 25(4):417423, Apr. 1954.

[HW58] A.J. Hatch and H.B. Williams. Multipacting modes of high-frequency gaseous breakdown. The Physical Review, 112(3):681-685, Nov. 1958. 
[JGME $\left.{ }^{+} 10\right]$ M. Jiménez, B. Gimeno, C. Miquel-Espanya, D. Raboso, S. Anza, C. Vicente, J. Gil, F. Quesada, A. Álvarez, M. Taroncher, et al. Analysis of the electromagnetic radiation generated by a multipactor discharge occurring within a microwave passive component. Journal of Physics D: Applied Physics, 43:395501, 2010 .

$\left[\mathrm{JMR}^{+} 12\right] \quad$ M. Jimenez, B.G. Martinez, D. Raboso, S. Anza, A. Alvarez, F. Quesada, V.E. Boria, C. Vicente, and J. Gil. Multimodal characterization of the multipactor effect in microwave waveguide components. Microwave and Wireless Components Letters, IEEE, 22(2):61 -63, feb. 2012 .

$\left[\mathrm{KBS}^{+} 02\right] \quad$ A. Kryazhev, M. Buyanova, V. Semenov, D. Anderson, M. Lisak, J.Puech, L. Lapierre, and J. Sombrin. Hybrid resonant modes of two-sided multipactor and transition to the polyphase regime. Physics of Plasmas, 11(9):4736-4743, Nov. 2002.

[KGV83] S. Kirkpatrick, C. D. Gelatt, and M. P. Vecchi. Optimization by simulated annealing. Science, 220(4598):671-680, 1983.

[Kis12] R. A. Kishek. Ping-pong modes: A new form of multipactor. Phys. Rev. Lett., 108:035003, Jan 2012.

[KL95] R. Kishek and Y.Y. Lau. Interaction of multipactor discharge and RF circuit. Physical Review Letters, 75(6):1218-1221, 1995.

$\left[\mathrm{KLA}^{+} 98\right] \quad$ R.A. Kishek, Y.Y. Lau, L.K. Ang, A. Valfells, and R.M. Gilgenbach. Multipactor discharge on metals and dielectrics: Historical review and recent theories. Physics of Plasmas, 5(5):2120-2126, May 1998.

$\left[\mathrm{KLS}^{+} 08\right] \quad$ I A Kossyi, G S Lukyanchikov, V E Semenov, E I Rakova, D Anderson, M Lisak, and J Puech. Polyphase (non-resonant) multipactor in rectangular waveguides. 
Journal of Physics D: Applied Physics, 41(6):065203 (8pp), 2008.

[Kry02] A. Kryazhev. Modeling and Simulations of Multipactor Discharges in Microwave Communication Lines. Master's thesis, Chalmers University of Technology, Göteborg, Sweden, 2002.

[KS69] W. F. Krolikowski and W. E. Spicer. Photoemission studies of the noble metals. i. copper. Phys. Rev., 185(3):882-900, Sep 1969.

[KS70] W. F. Krolikowski and W. E. Spicer. Photoemission studies of the noble metals. ii. gold. Phys. Rev. B, 1(2):478-487, Jan 1970.

[LD57] R. G. Lye and A. J. Dekker. Theory of secondary emission. The Physical Review, 107(4):977-981, Aug. 1957.

[Mar51] Nathan Marcuvitz. Waveguide handbook; 1951 ed. M.I.T. Radiat. Lab. McGraw-Hill, New York, NY, 1951.

[Mat03] M. Mattes. Contribution to the Electromagnetic Modeling and Simulation of Waveguide Networks Using Integral Equations and Adaptive Sampling, PhD Dissertation. PhD thesis, LEMA-EPFL, CH-1015, Lausanne, Switzerland, 2003. Thesis No. 2693.

$\left[\mathrm{MMS}^{+} 97\right]$ A. J. Marrison, R. May, J. D. Sanders, A. D. Dyne, A. D. Rawlins, and J. Petit. A study of multipaction in multicarrier RF components. AEA Technology for ESTEC Ref. AEA/TYKB/31761/01/RP/05, Culham, U.K., Jan. 1997.

$\left[\mathrm{MPD}^{+} 03\right]$ Ph. Mader, J. Puech, H. Dillenbourg, Ph. Lepeltier, L. Lapierre, and J. Sombrin. Study of the multipactor effect in multi-carrier operation inside space microwave components. In Proceedings of the 4 th International Workshop on Multipactor, RF and DC Corona and Passive Intermodulation in Space RF Hardware, pages 
489-491. ESTEC, Noordwijk, The Netherlands, Sept. 8-11 2003.

[Oli84] A. A. Oliner. Historical Perspectives on Microwave Field Theory. IEEE Transactions on Microwave Theory Techniques, 32:1022-1045, September 1984.

$\left[\mathrm{PBG}^{+} 09\right]$ A.M. Perez, V.E. Boria, B. Gimeno, S. Anza, C. Vicente, and J. Gil. Multipactor analysis in circular waveguides. Journal of Electromagnetic Waves and Applications, 23(11-12):1575-1583, 2009.

[PM98] Andrei D. Polyanin and Alexander V. Manzhirov. Handbook of Integral Equations. CRC Press LLC, 1998.

$\left[\mathrm{PSK}^{+} 03\right]$ K. S. Parikh, D. K. Singh, A. Praveen Kumar, Ms. Shilpi Rusia, and Mrs. K. Sangeetha. Multicarrier multipactor analysis of high power antenna TxTx diplexer for Satcom applications. In Proceedings of the 4th International Workshop on Multipactor, RF and DC Corona and Passive Intermodulation in Space $R F$ Hardware, pages 435-441. ESTEC, Noordwijk, The Netherlands, Sept. 8-11 2003.

[PSL05] K. Price, R. M. Storn, and J. A. Lampinen. Differential Evolution: A Practical Approach to Global Optimization. Springer, 2005.

$\left[\mathrm{PTV}^{+} 06\right] \quad$ A.M. Perez, C. Tienda, C. Vicente, A. Coves, G. Torregrosa, B. Gimeno, R. Barco, V.E. Boria, and D. Raboso. Multipactor analysis in coaxial waveguides for satellite applications using frequency-domain methods. In Microwave Symposium Digest, 2006. IEEE MTT-S International, pages 1045-1048, 2006.

$\left[\mathrm{PTV}^{+} 09\right] \quad$ A.M. Perez, C. Tienda, C. Vicente, S. Anza, J. Gil, B. Gimeno, V.E. Boria, and D. Raboso. Prediction of multipactor breakdown thresholds in coaxial transmission lines for traveling, standing, and mixed waves. IEEE Transactions on Plasma Science, 37(10):20312040, 2009. 
[PTVF07] William H. Press, Saul A. Teukolsky, William T. Vetterling, and Brian P. Flannery. Numerical Recipes, The Art of Scientific Computing. Cambridge University Press, 2007.

$\left[\mathrm{RAJ}^{+} 10\right]$ J. Rasch, D. Anderson, J.F. Johansson, M. Lisak, J. Puech, E. Rakova, and V.E. Semenov. Microwave multipactor breakdown between two cylinders. Plasma Science, IEEE Transactions on, 38(8):1997 -2005, aug. 2010.

[RCD95] S. Riyopoulos, D. Chernin, and D. Dialetis. Theory of electron multipactor in crossed fields. Physics of Plasmas, 2(8):3194-3212, Aug. 1995.

[Riy97] S. Riyopoulos. Multipactor saturation due to spacecharge-induced debunching. Phys. Plasmas, 4(5):14481462, May 1997.

$\left[\right.$ RLR $\left.^{+} 94\right] \quad$ N. Rozario, H. F. Lenzing, F. Reardon, M. S. Zarro, and C. G. Baran. Investigation of Telstar 4 spacecraft Kuband and C-band antenna components for multipactor breakdown. IEEE Transactions on Microwave Theory and Techniques, 42(4):558-564, Apr. 1994.

[RW95] D. Raboso and A. Woode. A new method of electron seeding used for accurately testing of multipactor transients. In 25th European Microwave Conference, volume 1, pages 190-193. Bologna, Italy, edited by Institute of Electrical and Electronics Engineers, IEEE, New-York, Sep. 1995.

$\left[\mathrm{SAG}^{+} 08\right] \quad$ E. Sorolla, S. Anza, B. Gimeno, A.M.P. Perez, C. Vicente, J. Gil, F.J.S. Prez, F.D. Quesada, A. Alvarez, and V.E. Boria. An analytical model to evaluate the radiated power spectrum of a multipactor discharge in a parallel-plate region. Electron Devices, IEEE Transactions on, 55(8):2252 -2258, aug. 2008. 
$\left[\mathrm{SAM}^{+}\right.$09] F.J.P. Soler, S. Anza, M. Mattes, C.M. Espana, F. Quesada, M. Jimenez, J. Gil, C. Vicente, J.R. Mosig, D. Raboso, V.E. Boria, B. Gimeno, and A. Alvarez-Melcon. Rigorous investigation of rf breakdown effects in high power microstrip passive circuits. In Microwave Symposium Digest, 2009. MTT '09. IEEE MTT-S International, pages $833-836$, june 2009.

$\left[\mathrm{SBA}^{+} 10\right]$ V. Semenov, M. Buyanova, D. Anderson, M. Lisak, R. Udiljak, and J. Puech. Multipactor in microwave transmission systems using quadrature phase-shift keying. Plasma Science, IEEE Transactions on, 38(4):915 -922 , april 2010.

$\left[\mathrm{SBS}^{+} 05\right]$ A. Sazontov, M. Buyanova, V. Semenov, E. Rakova, N. Vdovicheva, D. Anderson, M. Lisak, J. Puech, and L. Lapierre. Effect of emission velocity spread of secondary electrons in two-sided multipactor. Physics of Plasmas, 12(12):053102,8, May 2005.

[Sev05] R. Seviour. The role of elastic and inelastic electron reflection in multipactor discharges. IEEE Trans. Electron Devices, 52(8):1927-1930, Aug. 2005.

[She03] V. Shemelin. Generalized phase stability in multipacting, report SRF030904. Technical report, Cornell University, Superconducting Radio Frequency (SRF) group, 2003.

[SK01] V. Semenov and A. Kryazhev. Multipactor suppression in amplitude modulated radio frequency fields. Physics of Plasmas, 8(11):5034-5039, Nov. 2001.

[SPA] SPARK3D Multi-format High Power Simulation Tool. http://www.fest3d.com/.

[SPLL03] A.D. Sokolov, A.B. Pchelintsev, A.V. Loupilov, and V.A. Zalinkevich A. Lapenas. Beta and x-ray spectrometer for monitoring of beta-radiating nuclides. IEEE Transactions on Nuclear Science, 50(2):238-240, Apr 2003. 
$\left[\mathrm{SRA}^{+} 07\right]$ V. E. Semenov, E. I. Rakova, D. Anderson, M. Lisak, and J. Puech. Multipactor in rectangular waveguides. Physics of Plasmas, 14(3):033501, 2007.

[SRS ${ }^{+}$09] V E Semenov, E I Rakova, A G Sazontov, I M Nefedov, V I Pozdnyakova, I A Shereshevskii, D Anderson, M Lisak, and J Puech. Simulations of multipactor thresholds in shielded microstrip lines. Journal of Physics D: Applied Physics, 42(20):205204 (7pp), 2009.

$\left[\mathrm{SRU}^{+} 08\right]$ V. E. Semenov, E. Rakova, R. Udiljak, D. Anderson, M. Lisak, and J. Puech. Conformal mapping analysis of multipactor breakdown in waveguide irises. Physics of Plasmas, 15(3):033501, 2008.

[SRZ $\left.{ }^{+} 08\right]$ V. Semenov, E. Rakova, N. Zharova, D. Anderson, M. Lisak, and J. Puech. Simulations of the multipactor effect in hollow waveguides with wedge-shaped cross section. Plasma Science, IEEE Transactions on, 36(2):488-493, april 2008.

$\left[\mathrm{SSB}^{+} 05\right]$ A. Sazontov, V. Semenov, M. Buyanova, N. Vdovicheva, D. Anderson, M. Lisak, J. Puech, and L. Lapierre. Multipactor discharge on a dielectric surface: Statistical theory and simulation results. Physics of Plasmas, 12(9):093501, 2005.

[SSV08] A. G. Sazontov, V. A. Sazontov, and N. K. Vdovicheva. Multipactor breakdown prediction in a rectangular waveguide: statistical theory and simulation results. Contributions to Plasma Physics, 48(4):331-346, May 2008.

[Ste57] E. J. Sternglass. Theory of secondary electron emission by high-speed ions. The Physical Review, 108(1):1-12, Oct. 1957.

$\left[\mathrm{SVB}^{+} 03\right] \quad$ A. Sazontov, N. Vdovicheva, M. Buyanova, V. Semenov, D. Anderson, J. Puech, M. Lisak, and L. Lapierre. Simulations of multipactor zones taking 
into account realistic properties of secondary emission. In Proceedings of the 4th International Workshop on Multipactor, RF and DC Corona and Passive Intermodulation in Space RF Hardware. ESTEC, Noordwijk, The Netherlands, Sept. 8-11 2003.

$\left[\mathrm{SZA}^{+} 10\right]$ V. E. Semenov, N. A. Zharova, D. Anderson, M. Lisak, and J. Puech. Simulations of multipactor in circular waveguides. Physics of Plasmas, 17(12):123503, 2010.

$\left[\mathrm{SZU}^{+} 07\right] \quad$ V. E. Semenov, N. Zharova, R. Udiljak, D. Anderson, M. Lisak, and J. Puech. Multipactor in a coaxial transmission line. ii. particle-in-cell simulations. Physics of Plasmas, 14(3):033509, 2007.

$\left[\mathrm{TCB}^{+} 05\right] \quad$ G. Torregrosa, A. Coves, A. A. San Blas, A. M. Pérez, C.. P. Vicente, B. Gimeno, and V. E. Boria. Analysis of multipactor effect in dielectric-loaded waveguides. In Proceedings of the 5th International Workshop on Multipactor, RF and DC Corona and Passive Intermodulation in Space RF Hardware, pages 11-17. ESTEC, Noordwijk, The Netherlands, Sept. 12-14 2005.

$\left[\mathrm{TCV}^{+} 06\right]$ G. Torregrosa, A. Coves, C.P. Vicente, A.M. Perez, B. Gimeno, and V.E. Boria. Time evolution of an electron discharge in a parallel-plate dielectric-loaded waveguide. Electron Device Letters, IEEE, 27(7):619$621,2006$.

$\left[\mathrm{TPCM}^{+} 10\right]$ G. Torregrosa-Penalva, A. Coves, B.G. Martinez, I. Montero, C. Vicente, and V.E. Boria. Multipactor susceptibility charts of a parallel-plate dielectric-loaded waveguide. Electron Devices, IEEE Transactions on, 57(5):1160-1166, 2010.

$\left[\mathrm{UAI}^{+} 03\right]$ R. Udiljak, D. Anderson, P. Ingvarson, U. Jordan, U. Jostell, L. Lapierre, G. Li, M. Lisak, J. Puech, and J. Sombrin. New method for detection of multipaction. IEEE Transactions on Plasma Science, 31(31):396-404, Jun. 2003. 
$\left[\mathrm{UAL}^{+}\right.$07a] R. Udiljak, D. Anderson, M. Lisak, J. Puech, and V.E. Semenov. Multipactor in a waveguide iris. Plasma Science, IEEE Transactions on, 35(2):388 -395, april 2007.

$\left[\mathrm{UAL}^{+} 07 \mathrm{~b}\right] \quad$ R. Udiljak, D. Anderson, M. Lisak, V. E. Semenov, and J. Puech. Multipactor in a coaxial transmission line. i. analytical study. Physics of Plasmas, 14(3):033508, 2007.

[Vau88] J.R.M. Vaughan. Multipactor. IEEE Trans. Electron Devices, 35:1172-1180, Jul. 1988.

[Vau89] J.R.M. Vaughan. A new formula for Secondary Emission Yield. IEEE Trans. Electron Devices, 36(9):19631967, Sept. 1989.

[Ver05] J.P. Verboncoeur. Particle simulation of plasmas: review and advances. Plasma Physics and Controlled Fusion, 47(5A):A231-A260, Apr. 2005.

$\left[\mathrm{VMW}^{+} 05 \mathrm{a}\right]$ C. Vicente, M. Mattes, D. Wolk, H. L. Hartnagel, J. R. Mosig, and D. Raboso. FEST3D: A simulation tool for multipactor prediction. In Proceedings of the 5th International Workshop on Multipactor, $R F$ and $D C$ Corona and Passive Intermodulation in Space RF Hardware, pages 11-17. ESTEC, Noordwijk, The Netherlands, Sept. 12-14 2005.

[VMW ${ }^{+}$05b] C. Vicente, M. Mattes, D. Wolk, H. L. Hartnagel, J. R. Mosig, and D. Raboso. Multipactor breakdown prediction in rectangular waveguide based components. In Microwave Symposium Digest, 2005 IEEE MTT-S International, volume 2, pages 1055-1058. Long Beach, California, USA, edited by Institute of Electrical and Electronics Engineers, IEEE, New-York, Jun. 12-17 2005 .

$\left[\mathrm{VMW}^{+} 05 \mathrm{c}\right]$ C. Vicente, M. Mattes, D. Wolk, H. L. Hartnagel, J. R. Mosig, and D. Raboso. Multipactor breakdown prediction in rectangular waveguide based components. In 
Microwave Symposium Digest, IEEE MTT-S International, volume 2, pages 1055-1058. Long Beach, California, USA, Jun. 12-17 2005.

[VSS04] N. K. Vdovicheva, A. G. Sazontov, and V. E. Semenov. Statistical theory of two-sided multipactor. $R a$ diophysics and Quantum Electronics, 47(8), 2004.

[VSSS06] N. K. Vdovicheva, A. G. Sazontov, V. A. Sazontov, and V. E. Semenov. Influence of the angular anisotropy of secondary emission on the characteristics of a two-sided multipactor. Radiophysics and Quantum Electronics, 49(5):368-376, May 2006.

[Wac64] H. M. Wachowski. Breakdown in waveguides due to the multipactor effect. Technical Report TDR-269(9990)-5, El Segundo Technical Operations AEROSPACE CORPORATION, El Segundo, California, May 1964.

[WJ89] A. Woode and J.Petit. Diagnostic investigations into the multipactor effect, susceptibility zone measurements and parameters affecting a discharge. Technical report, ESTEC working paper No. 1556, Noordwijk, Nov. 1989.

[Woo68] R. Woo. Multipacting discharges between coaxial electrodes. Journal of Applied Physics, 39(3):1528-1533, Feb. 1968.

[WSS00] D. Wolk, D. Schmitt, and T. Schlipf. A novel approach for calculating the multipaction threshold in multicarrier operation. In Proceedings of the 3rd International Workshop on Multipactor, RF and DC Corona and Passive Intermodulation in Space RF Hardware, pages 85-91. ESTEC, Noordwijk, The Netherlands, Sept. 4-6 2000 .

[ZGPdD03] P. Zuccarello, A. González, G. Piñero, and M. de Diego. Software tool for evaluation of the amplitude and time statistics of digitally modulated multicarrier systems 
for space communications. In Proceedings of the 4 th International Workshop on Multipactor, RF and DC Corona and Passive Intermodulation in Space RF Hardware, pages 469-473. ESTEC, Noordwijk, The Netherlands, Sept. 8-11 2003. 